\title{
A Predistortion Circuit for Direct Modulated Lasers for Radio over Fiber Applications
}

\author{
by \\ Zhan Xu, B.Eng., M.A.Sc. \\ A thesis submitted to \\ the Faculty of Graduate Studies and Research \\ in partial fulfilment of the requirement for the degree of
}

\section{Doctor of Philosophy}

\author{
in \\ Electrical Engineering \\ Ottawa-Carleton Institute for Electrical and Computer Engineering \\ Department of Electronics \\ Carleton University \\ Ottawa, Ontario, Canada
}

August 2010

Copyright (c) Zhan Xu, 2010 


$\begin{array}{ll}\begin{array}{l}\text { Library and Archives } \\ \text { Canada }\end{array} & \begin{array}{l}\text { Bibliotheque et } \\ \text { Archives Canada }\end{array} \\ \begin{array}{l}\text { Published Heritage } \\ \text { Branch }\end{array} & \begin{array}{l}\text { Direction du } \\ \text { Patrimoine de l'édition }\end{array} \\ \begin{array}{l}\text { 395 Wellington Street } \\ \text { Ottawa ON K1A ON4 } \\ \text { Canada }\end{array} & \begin{array}{l}\text { 395, rue Wellington } \\ \text { Ottawa ON K1A ON4 } \\ \text { Canada }\end{array}\end{array}$

Your file Votre référence

ISBN: 978-0-494-70563-6

Our file Notre référence

ISBN: 978-0-494-70563-6

\section{NOTICE:}

The author has granted a nonexclusive license allowing Library and Archives Canada to reproduce, publish, archive, preserve, conserve, communicate to the public by telecommunication or on the Internet, loan, distribute and sell theses worldwide, for commercial or noncommercial purposes, in microform, paper, electronic and/or any other formats.

The author retains copyright ownership and moral rights in this thesis. Neither the thesis nor substantial extracts from it may be printed or otherwise reproduced without the author's permission.

\section{AVIS:}

L'auteur a accordé une licence non exclusive permettant à la Bibliothèque et Archives Canada de reproduire, publier, archiver, sauvegarder, conserver, transmettre au public par télécommunication ou par l'Internet, prêter, distribuer et vendre des thèses partout dans le monde, à des fins commerciales ou autres, sur support microforme, papier, électronique et/ou autres formats.

L'auteur conserve la propriété du droit d'auteur et des droits moraux qui protège cette thèse. $\mathrm{Ni}$ la thèse ni des extraits substantiels de celle-ci ne doivent être imprimés ou autrement reproduits sans son autorisation.
In compliance with the Canadian Privacy Act some supporting forms may have been removed from this thesis.

While these forms may be included in the document page count, their removal does not represent any loss of content from the thesis.
Conformément à la loi canadienne sur la protection de la vie privée, quelques formulaires secondaires ont été enlevés de cette thèse.

Bien que ces formulaires aient inclus dans la pagination, il n'y aura aucun contenu manquant. 


\section{CARLETON UNIVERSITY}

Date: August 2010

Author: Zhan Xu

Title: $\quad$ A Predistortion Circuit for Direct Modulated Lasers for Radio over Fiber Applications

Department: Electronics

Degree: Ph.D. Convocation: December Year: 2010

I hereby declare that I am the sole author of this thesis. I authorize Carleton University to lend this thesis to other institutions or individuals for the purpose of scholarly research.

\section{Signature of Author}

I further authorize Carleton University to reproduce this thesis by photocopying or by other means, in total or in part, at the request of other institutions or individuals for the purpose of scholarly research.

Signature of Author 


\section{To Yilin, Adaia and Yanli}

\section{Now faith is being sure of what we hope for and certain of what we do not see-Hebrews 11:1}




\section{Abstract}

In radio over fiber systems, a linear optical transmitter is desirable in order to achieve an extended signal dynamic range. This thesis introduces and discusses a predistortioncompensation linearization technique to improve the optical transmitter's linearity. In order to compensate for the second and the third order laser distortions, the predistorter's quadratic and cubic law circuits are required to work at up to 2 or 3 times the carrier frequency. In this thesis, an alternative design approach is proposed to relieve the bandwidth requirement of the predistortion circuits. This approach uses multiple tank circuits to approximate the frequency profile shaping filters in the frequency band of interest. At the same time, tunability is provided to account for the changes of the laser's distortion behavior due to component variations, thermal effects and aging. In order to tune the predistortion circuit adaptively, a calibration algorithm was proposed and implemented for minimizing the laser distortion. This algorithm is based on multiple variable feedback, which directly processes the RF signal and does not require a DSP unit or an optimization routine. This suggests a potential complete analog implementation with less circuit complexity. A prototype predistorter IC has been designed using $0.18 \mu \mathrm{m}$ CMOS technology. It operates near $2 \mathrm{GHz}$ for demonstration purpose and has more than $300 \mathrm{MHz}$ linearized bandwidth. 5 to $15 \mathrm{~dB}$ reduction of the second harmonic distortion and the third order intermodulation distortion can be obtained. 


\section{Acknowledgement}

I would like to express my deep appreciation and gratitude to my supervisor, Dr. Leonard MacEachern, for his guidance, support and great patience. Thanks for his many critical suggestions, which are effective and efficient. They make this work practically done.

Thanks to my wife Yanli, and my daughters, Yilin and Adaia, for their patience, love and great sacrifice. I would like to thank my parents and my brother who have continued to show great interest and support in what I am doing.

I would like to thank all my friends and colleagues for their support and input over the years. All of those I will never forget. 


\title{
Table of Contents
}

\begin{abstract}
$\quad$ v
Acknowledgement $\quad$ vi

Table of Contents vii

List of Tables $\quad$ xi

List of Figures $\quad$ xii

1 Introduction $\quad 1$

1.1 Background . . . . . . . . . . . . . . . . . 2

1.2 Thesis Motivation . . . . . . . . . . . . . . . . . . . . 3

1.3 Thesis Objective . . . . . . . . . . . . . . . . 3

1.4 Thesis Contribution and Publications . . . . . . . . . . . . 4

1.5 Thesis Organization . . . . . . . . . . . . . . . . 5
\end{abstract}

2 Laser Modeling and Linearization Techniques $\quad 7$

2.1 Laser Principles and Laser Oscillation . . . . . . . . . . . . . . . 7

2.1.1 Laser Principles . . . . . . . . . . . . . . . . . . . . 7

2.1.2 Explanation of Laser Oscillation . . . . . . . . . . . . 8

2.2 Laser Model . . . . . . . . . . . . . . . . . . . . . . . . . . . . . . 14

2.2 .1 Rate Equations . . . . . . . . . . . . . . . . . . . . 14

2.2 .2 Rate Equations Based Model in ADS . . . . . . . . . . . . 15

2.3 Analysis of Laser Diode Behavior . . . . . . . . . . . . . . . . . . 19

2.3 .1 DC Analysis . . . . . . . . . . . . . . . . . . . . . . 19

2.3 .2 Small Signal Analysis . . . . . . . . . . . . . . . . . . . . . . . . 22

2.3 .3 Large Signal Nonlinearity . . . . . . . . . . . . . . . . . . . . . . . . . . . . . . . . . . 32

2.4 Laser Inverse Model . . . . . . . . . . . . . . . . . . . . . . . . . . . . 32

2.5 Inverse Model Based Laser Model . . . . . . . . . . . . . . . . . . . 33

2.6 Review of Laser Linearization Techniques . . . . . . . . . . . . . . . . 37 
2.6 .1 Optoelectronic Feedback . . . . . . . . . . . . . . . . . . . . 37

2.6 .2 Optical Feed-forward . . . . . . . . . . . . . . . . . . . . 38

2.6 .3 Phase Shift Linearization . . . . . . . . . . . . . . . . . . . . 39

2.6 .4 External Light Injection . . . . . . . . . . . . . . . . . . 39

2.6.5 Postdistortion . . . . . . . . . . . . . . . . . 40

2.6 .6 Predistortion . . . . . . . . . . . . . . . 41

2.7 Predistortion Challenges . . . . . . . . . . . . . . . . . . . . 43

2.8 Summary . . . . . . . . . . . . . . . . . . . . . 44

3 Predistortion Function Using Multiple Tank Approach 45

3.1 Predistorter Model . . . . . . . . . . . . . . . . . . . 45

3.2 Proposed Multiple Tank Technique . . . . . . . . . . . . . . . . . . . 49

3.3 Tank Gain Calculation . . . . . . . . . . . . . . . . . 52

3.3.1 Based on Optimization . . . . . . . . . . . . . 53

3.3 .2 Based on Point Matching . . . . . . . . . . . . . . . 55

3.4 Mismatch and Distortion . . . . . . . . . . . . . . . 56

3.4.1 Tank Circuit Parameters Optimization . . . . . . . . . . . 56

$3.4 .22^{\text {nd }}$ Order Distortion $\ldots \ldots \ldots \ldots \ldots$

$3.4 .33^{\text {rd }}$ Order Distortion . . . . . . . . . . . . . . . . . . 58

3.5 Sensitivity, Resolution, and Tuning Range . . . . . . . . . . . . 59

3.6 Noise Figure Consideration . . . . . . . . . . . . . . . . . . . 60

3.7 Summary . . . . . . . . . . . . . . . . . . . . . 61

4 Predistortion Circuit Design in CMOS $\quad 62$

4.1 Circuit Building Blocks . . . . . . . . . . . . . . . . . . . . 62

$4.1 .1 X^{2}$ Component . . . . . . . . . . . . . . 63

4.1.2 $X^{3}$ Component and Tunable Gain Cell . . . . . . . . . . . . . . 67

4.1 .3 Laser Driver and Summing . . . . . . . . . . . . . . . . . . . . 74

4.2 Design Example and Simulation Results . . . . . . . . . . . . . 80

4.2 .1 Predistorter Model Parameters . . . . . . . . . . . . . . 80

4.2 .2 Frequency Band Allocation . . . . . . . . . . . . . . . . . . . . 82

4.2 .3 Distortion Generator Calculation . . . . . . . . . . . . . . . . 83

4.2 .4 Laser Driver Modeling . . . . . . . . . . . . . . . . . . . 84

4.2 .5 Tank Circuits Optimization . . . . . . . . . . . . . 87

4.2 .6 Overall Circuit and Simulation Results . . . . . . . . . . . . . . 92

4.3 Design Methodology . . . . . . . . . . . . . . . . . . . 96

4.4 Summary . . . . . . . . . . . . . . . . . . . 98 
5 Wideband Balun for Even-mode Distortion Cancelation 99

5.1 Basic Marchand Balun . . . . . . . . . . . . . . . . . . . . . 99

5.2 Wideband Marchand Balun . . . . . . . . . . . . . . . 102

5.2 .1 Derivation of Design Equations . . . . . . . . . . . . . . . 104

5.2.2 Comparison of Higher Order Marchand Baluns . . . . . . . . . . . 112

5.3 Fully Matched Wideband Marchand Balun . . . . . . . . . . . . . . . . 115

5.3 .1 Proposed Even Mode Matching . . . . . . . . . . . . . . . . 116

5.3 .2 Coupled Line Models . . . . . . . . . . . . . . . . . . . 118

5.3.3 Design Formulas, One Section $(\mathrm{M}=1) \ldots \ldots . . \ldots . . \ldots 122$

5.3.4 Design Procedure, Multiple Sections $(M \geq 2) \ldots \ldots . . \ldots$.

5.4 Measurement Results . . . . . . . . . . . . . . . . . . . 125

5.5 Summary . . . . . . . . . . . . . . . . . . . 130

6 Adaptive Calibration and Measurement Results $\quad 132$

6.1 General Considerations . . . . . . . . . . . . . . . . . . . . . . . . . . . . . . . . 132

6.1 .1 Optimization . . . . . . . . . . . . . . . . 133

6.1 .2 Iteration (Feedback) . . . . . . . . . . . . . . . . . . 134

6.2 Adaptive Calibration using Multiple Variable Feedback . . . . . . . . . . . 134

6.3 IC Layout and Micrograph . . . . . . . . . . . . . . . . . . . . . . . . . . . . . . . . . . . . . . .

6.4 Chip Bonding and Test Board Design . . . . . . . . . . . . . . 147

6.5 Test Setup and Equipments . . . . . . . . . . . . . . . . . . 149

6.6 Measurement . . . . . . . . . . . . . . . . . . 150

6.6 .1 Input Signal . . . . . . . . . . . . . . . . . . 150

6.6.2 Responses of the Tank Circuits . . . . . . . . . . . . . . . . 153

6.6 .3 Adaptive Calibration . . . . . . . . . . . . . . . . . . . 153

K Matrix and Gain Saturation . . . . . . . . . . . . . . 153

Relaxation Factor . . . . . . . . . . . . . . . . 157

Tracking . . . . . . . . . . . . . . . . . . 158

Frequency and Power Sweep . . . . . . . . . . . . . . 164

6.7 Summary . . . . . . . . . . . . . . . . . . . . 168

$\begin{array}{llr}7 \text { Conclusions } & 169\end{array}$

7.1 Conclusions . . . . . . . . . . . . . . . . . . . . . 169

7.2 Recommendations for Future Research . . . . . . . . . . . . 170

A Laser Inverse Model Constants $\quad 174$

B Tank Circuit Optimization Contour Plots 176

C Relationship between the Normal Mode Parameters and the Distributed $\begin{array}{lr}\text { Parameters of the Coupled Transmission Line } & 184\end{array}$ 
D Using Volterra Transfer Function to Calculate the Multi-tone Response of a Nonlinear System

E MatLab Code of the Adaptive Calibration Algorithm 188

$\begin{array}{lr}\text { Bibliography } & 197\end{array}$ 


\section{List of Tables}

2.1 Laser model parameters . . . . . . . . . . . . . . . . . . . . 17

4.1 Predistorter model parameters . . . . . . . . . . . . . . 80

4.2 Laser driver frequency response polynomial fit parameters . . . . . . . . 86

5.I Basic quarter-wave transmission line elements and their chain scattering parameters . . . . . . . . . . . . . . . . . 107

5.2 Design polynomials for $1^{\text {st }}$ order pre-warping. . . . . . . . . . . 110

5.3 Design polynomials for $2^{\text {nd }}$ order pre-warping. . . . . . . . . . . . 111

5.4 Design polynomials for $3^{r d}$ order pre-warping. . . . . . . . . . . . . 111

5.5 Design polynomials for $4^{\text {th }}$ order pre-warping. . . . . . . . . . . . . 112

5.6 Z-matrix for a general coupled line section. . . . . . . . . . . . . . . . 119

5.7 Simplified Z-matrix for uniplanar case (assume $\left.\phi_{c} \approx \phi_{\pi} \approx \phi\right) \ldots \ldots \ldots$

5.8 Simplified Z-matrix for two-layer planar case $\left(R_{\pi} \approx 0\right) \ldots \ldots \ldots$

5.9 Characteristic impedances of the designed balun . . . . . . . . . 126 


\section{List of Figures}

2.1 The three key processes in laser operation $\ldots \ldots \ldots \ldots$

2.2 Schematic longitudinal view of a Fabry-Perot laser . . . . . . . . . . . 9

2.3 Gain spectrum . . . . . . . . . . . . . . . . 11

2.4 Illustration of the carrier distribution of a p-n junction . . . . . . . . 11

2.5 Laser output spectrum . . . . . . . . . . . . . . . . . . . 13

2.6 Symbolic laser model defined in ADS . . . . . . . . . . . 18

2.7 Equivalent circuit of the laser rate equations . . . . . . . . . . . . . 19

2.8 Laser model dc response . . . . . . . . . . . . . . . . . . . . . 21

2.9 Small signal equivalent circuit of the laser rate equations . . . . . . . . 22

2.10 Laser model ac response . . . . . . . . . . . . . . . . . . . . 24

2.11 Equivalent circuit of the laser rate equations . . . . . . . . . . . . 25

2.12 Linearized circuit for calculating first order Volterra kernel . . . . . . . 26

2.13 Equivalent circuit for calculating second or third order Volterra kernel . . . 26

2.14 Linear component of laser output . . . . . . . . . . . . . . 30

2.15 Second order harmonic distortion of laser output . . . . . . . . . . . . . 30

2.16 Third order harmonic distortion of laser output . . . . . . . . . . . . . 31

2.17 Third order intermodulation distortion of laser output . . . . . . . . . . 31

2.18 Block diagram of laser inverse model . . . . . . . . . . . . . . 33

2.19 A cascade of two stages. . . . . . . . . . . . . . . . . 33

2.20 Linear component of laser output . . . . . . . . . . . . . . 35

2.21 Second order harmonic distortion of laser output . . . . . . . . . 35

2.22 Third order harmonic distortion of laser output . . . . . . . . . . 36

2.23 Third order intermodulation distortion of laser output . . . . . . . . . 36 
2.24 Optoelectronic feedback linearization . . . . . . . . . . . . . 37

2.25 Optical feed-forward linearization . . . . . . . . . . . . . 38

2.26 Phase shift modulation linearization . . . . . . . . . . . . . . . 39

2.27 External light injection . . . . . . . . . . . . . . . . . . . 40

2.28 Optical postdistortion . . . . . . . . . . . . . 40

2.29 Electrical postdistortion . . . . . . . . . . . . . . . 40

2.30 Predistortion . . . . . . . . . . . . . . . . . . 42

3.1 Block diagram of the laser predistorter . . . . . . . . . . . . . 46

3.2 Output spectrum of $X X^{2}$ and $X^{3}$ components . . . . . . . . . . 48

3.3 Predistortion principle . . . . . . . . . . . . . 48

3.4 Laser predistortion principle . . . . . . . . . . . . . . 48

3.5 Distortion cancelation in a cascade of two stages. . . . . . . . . . . . . 49

3.6 LC tank circuit . . . . . . . . . . . . . . . . . . . . . 50

3.7 Amplitude and phase interpolation using two tank circuits . . . . . . . 51

3.8 Multi-tank approach for frequency profile shaping . . . . . . . . . . 52

3.9 Predistorter model using multi-tank approach . . . . . . . . . . 53

3.10 Illustration of bandwidth and spacing . . . . . . . . . . . 57

4.1 NMOS device . . . . . . . . . . . . . . . . . . . . . . 63

4.2 Simulated input-output (V-I) characteristic of an nmos transistor . . . . 64

4.3 Coefficients of linear, square and cubic terms . . . . . . . . . . 65

$4.4 X^{2}$ distortion generator . . . . . . . . . . . . 66

4.5 A differential pair . . . . . . . . . . . . . . . 67

4.6 Linear and cubic coefficients of the differential pair . . . . . . . . . . 69

4.7 Tunable linear and cubic gain cell . . . . . . . . . . . . . . 70

4.8 Third order distortion cancelation using Gilbert cell . . . . . . . . . . 71

4.9 Linear and cubic coefficients of the tunable gain cell . . . . . . . . . . . . 72

4.10 Circuit of a digitally tunable gain cell . . . . . . . . . . . . . . 74

4.11 Laser driver . . . . . . . . . . . . . . . . . . . . . . . 75

4.12 Multi-tanh linearization principle . . . . . . . . . . . 75 
4.13 Linear and cubic coefficients of (a) multi-tanh linearized pair and (b) simple differential pair . . . . . . . . . . . . . . . . 77

4.14 (a) Effects of offset voltage on multi-tanh linearized pair and (b) effects of bias current on simple differential pair f . . . . . . . . . . 78

4.15 Current combining circuit . . . . . . . . . . . . . . . 79

4.16 Ideal predistortion results . . . . . . . . . . . . . . . 81

4.17 Obtaining the coefficients of distortion generators . . . . . . . . . 83

4.18 AC response of the $X^{2}$ circuit $\ldots \ldots \ldots \ldots \ldots$

4.19 AC response of laser driver circuit . . . . . . . . . . . . . . . . . 86

4.20 Block diagram of the predistortion system. f . . . . . . . . . . 87

4.21 Contour plot of mismatch induced distortion using 1 ns delay . . . . . . . . 90

4.22 Simulation results using ideal tank circuits . . . . . . . . . . . . . . 91

4.23 Overall circuit . . . . . . . . . . . . . . . . . . . . . 93

4.24 Circuit simulation results . . . . . . . . . . . . . . . . . 95

4.25 Design flow chart . . . . . . . . . . . . . . . . . . 97

5.1 Basic (a) uncompensated and (b) compensated Marchand balun and their equivalent circuits. . . . . . . . . . . . . . . . . 100

5.2 Bisected equivalent circuits of (a) uncompensated and (b) compensated Marchand balun for differential mode operation. . . . . . . . . . . . . . 100

5.3 Possible balun configurations using open or short stubs and unit elements (b and $\mathrm{d}$ are topologies with redundancy) . . . . . . . . . . . . . . 103

5.4 Redundancy illustrated by the equivalency of Kuroda identities. . . . . . . 104

5.5 Topologies of the proposed multiple stage Marchand balun in the ascending sequence of warping orders (number of short or open stubs) . . . . . . . 105

5.6 Prewarping to obtain an equal-ripple response. . . . . . . . . . . . . . . . 109

5.7 Comparison of 5-element baluns with $1^{\text {st }}, 2^{\text {nd }}$, and $3^{\text {rd }}$ order prewarping. . 113

5.8 Comparison of 6-element baluns with $1^{\text {st }}, 2^{\text {nd }}, 3^{\text {rd }}$, and $4^{\text {th }}$ order prewarping. 113

5.9 Comparison of 7 -element baluns with $1^{\text {st }}, 2^{\text {nd }}, 3^{\text {rd }}$, and $4^{\text {th }}$ order prewarping. 113

5.10 Marchand balun bisected equivalent circuit for even-mode excitations. . . . 115 
5.11 (a) Proposed coupled-line configuration for even-mode matching and its bisected equivalent circuit for (b) odd-mode and (c) even-mode excitations. . 116

5.12 (a) Proposed fully matched Marchand balun and its bisected equivalent circuit for (b) odd-mode and (c) even-mode excitations. . . . . . . . . . 117

5.13 (a) A general uniformly coupled line (b) $\pi$-mode and (c) $c$-mode propagation. 118

5.14 Lumped equivalent circuit of coupled asymmetrical transmission lines of differential length $d z[1] \ldots \ldots$. . . . . . . . . . . . . . . . . . . . . . . . 119

5.15 Coupled-line structures on a two-side substrate and their simplified models:

(a) and (c) for uniplanar case; (b) and (d) for two-layer planar case. . . . . 121

5.16 Two-port model of the even-mode matching section for even-mode excitation. 121

5.17 Even-mode network model of a m-section balun. . . . . . . . . . . . . . 122

5.18 Common mode reflection coefficient. . . . . . . . . . . . . . . . . 124

5.19 Modified even-mode topology. . . . . . . . . . . . . . . . . 126

5.20 PCB layout of the designed balun. . . . . . . . . . . . . . . . 127

5.21 A zoomed-in view of the layout. . . . . . . . . . . . . . 127

5.22 Photograph of the fabricated balun. . . . . . . . . . . . . . . . 128

5.23 Measured $S_{11}, S_{21}$ and $S_{31}$ compared with EM simulated results. . . . . . 129

5.24 Measured $S_{22}, S_{33}$ and $S_{23}$ compared with EM simulated results. . . . . 130

5.25 Measured phase and amplitude imbalance compared with EM simulated results. . . . . . . . . . . . . . . . . . . 131

6.1 Newton-Raphson iteration . . . . . . . . . . . . . . . . 135

6.2 Fixed-point iteration . . . . . . . . . . . . . . . 136

6.3 Block diagram of the predistorter IC and the feedback control. . . . . . . 137

6.4 Multiple variable feedback. . . . . . . . . . . . . . . . 137

6.5 Layout of the $X^{2}$ component. . . . . . . . . . . . . . . . . . . . 143

6.6 Layout of the tunable gain tank circuit and the laser driver. . . . . . . . 144

6.7 Layout of current summing circuit. . . . . . . . . . . . . . . 145

6.8 Complete layout of the predistorter IC . . . . . . . . . . . . . 145

6.9 Micrograph of the fabricated predistorter IC . . . . . . . . . . . . . . 146

6.10 Photograph of the test board with a zoomed-in view of the chip bonding area. 147 
6.11 Picture of the test board mounted on a bread-board. . . . . . . . . . . 148

6.12 Test setup. . . . . . . . . . . . . . . . . . . . . . . 149

6.13 Spectrum analyzer screenshot showing the two-tone input signal. . . . . . 151

6.14 Measured results showing the tank circuit alignment and tuning. The tuning voltages were increased from 1.1 to $1.5 \mathrm{~V}$ in $0.01 \mathrm{~V}$ step to demonstrate the fine tuning capability. . . . . . . . . . . . . . . . . 152

6.15 Measured results showing the calibration process with $5 \mathrm{~mA} R F$ input to the laser. . . . . . . . . . . . . . . . . . . . . . . 155

6.16 Measured results showing the calibration process with $3 \mathrm{~mA} \mathrm{RF}$ input to the laser. . . . . . . . . . . . . . . . . . . . . . . 156

6.17 Conceptual illustration of the feedback instability due to a large relaxation factor. . . . . . . . . . . . . . . . . 157

6.18 Measured results showing the calibration process with $\alpha_{1,2,3,4}=0.06$ and

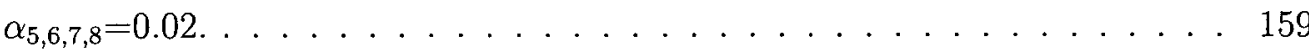

6.19 Measured results showing the calibration process with $\alpha_{1,2,3,4}=0.06$ and $\alpha_{5,6,7,8}=0.06 \ldots \ldots \ldots \ldots \ldots \ldots$. . . . . . . . . . . . . . . . . . . . . .

6.20 Measured results showing the capability of tracking to the change of bias current. . . . . . . . . . . . . . . . . . 162

6.21 Long term observation of the settling after tracking for (a) IM3 distortion and (b) HD2 distortion. . . . . . . . . . . . . . . . . . . . 163

6.22 IM3 and HD2 distortion versus frequency. . . . . . . . . . . . . . 165

6.23 IM3 and HD2 distortion versus output optical intensity. . . . . . . . . . 166

6.24 IM2 distortion measurement results. . . . . . . . . . . . . . . . . . . . 167

7.1 Possible circuit implementation of the feedback control algorithm. . . . . . 172

B.1 Tank circuit parameters optimization for different linear path delays . . . . 177

B.2 Tank circuit parameters optimization for different linear path delays . . . . 178

B.3 Tank circuit parameters optimization for different linear path delays . . . . 179

B.4 Tank circuit parameters optimization for different linear path delays . . . . 180

B.5 Tank circuit parameters optimization for different linear path delays . . . . 181

B.6 Tank circuit parameters optimization for different linear path delays . . . . 182 
B.7 Tank circuit parameters optimization for different linear path delays . . . . 183

D.1 A $p^{\text {th }}$ order nonlinear system . . . . . . . . . . . . . 186 


\section{Chapter 1}

\section{Introduction}

With the explosive growth of wireless communications, broadband air interfaces are needed to accommodate high user density, high speed data transport and fast internet applications (multimedia services etc.) and tremendous increase in channel bandwidth will be required for the future wireless networks. In order to increase the network capacity with the allocated frequency bandwidth, frequencies are reused for different transmissions among the radio cells in a cellular network. Deployment of a large number of radio cells (micro-cells and pico-cells) in the same area, will result in a great increase of available channel bandwidth and user numbers. The huge numbers of cell interconnections can be carried out effectively by optical fiber. Radio over Fiber (RoF) refers to the technology whereby light is modulated by a radio signal and transmitted over an optical fiber link to facilitate the wireless access. The use of optical fibers to transmit RF signals has many advantages over RF cables, such as low attenuation, high bandwidth, light weight and low cost. As a convenient way to distribute RF signals to the radio cells for wireless communications, RoF offers several advantages over conventional optical communication systems: dynamic allocation of carrier frequencies, enhanced micro-cellular coverage and capacity, low complexity of base station design, and hence low-cost base station deployment [2]. 


\subsection{Background}

Various methods exist for the transmission of radio frequency $(R F)$ signals over optical fiber, such as direct intensity modulation, external intensity modulation and remote heterodyne. In principle, direct modulation is the simplest solution, where the optical intensity is modulated by directly modulating the laser driving current. The performance of direct modulation is comparable to that of external modulation for the signal frequency up to $10 \mathrm{GHz}$. Due to the modulation bandwidth of the laser, at higher frequencies, an external modulator is applied. For the signal frequency over a few tens of $\mathrm{GHz}$, optical remote heterodyne is applied due to the fiber dispersion [2].

In the direct modulation method, the input current of a semiconductor laser is directly modulated by the information-bearing RF signal. Due to the nonlinear relationship between the input current and the output optical power of the semiconductor laser, distortion is introduced to the transmission system. Linearity is an important consideration in the design of an analog light-wave transmission system. For delivering a service with satisfactory quality, it is mandatory to keep the distortion below a certain level, for example, the CATV application requires a carrier to distortion ratio better than 50dB; GSM picocellular and micro-cellular requires a satisfactory dynamic range of $40-55 \mathrm{~dB}$ and $80-90 \mathrm{~dB}$ respectively $[3,4]$. Improved fabrication techniques and device structures for linear lasers have led to many successful applications, but fabricating these lasers is a more expensive task. The alternative approach is using linearization techniques to reduce the laser distortion. A variety of those techniques have been proposed in earlier research [5-19] and those techniques can be classified as: optoelectronic feedback, optical feed-forward [5-10], postdistortion [11-13] and pre-distortion [14-19]. Published results show that optoelectronic feedback reduces the $2^{\text {nd }}$ and the $3^{\text {rd }}$ harmonic distortion by $10-20 \mathrm{~dB}[20,21]$, that optical feed-forward reduces the $2^{\text {nd }}$ and the $3^{\text {rd }}$ order harmonic distortion by $14-25 \mathrm{~dB}[8,22]$, postdistortion reduces the $2^{\text {nd }}$ and the $3^{\text {rd }}$ order harmonic distortion by $10 \mathrm{~dB}$ and pre-distortion reduces the $2^{\text {nd }}$ and the $3^{\text {rd }}$ order harmonic distortion by $15 \mathrm{~dB}$ [23]. Our previous research shows the potential of more than $50 \mathrm{~dB}$ reduction of the $2^{\text {nd }}$ and the $3^{\text {rd }}$ order harmonic 
distortion using adaptive pre-distortion [24].

\subsection{Thesis Motivation}

The synergy of wireless and optical fiber communication employing radio-over-fiber technology demands a highly linear analog optical link. There are two additional major methods to reduce the nonlinear distortions generated by lasers: feedforward compensation and predistortion $[5,25]$. Feedforward compensation also reduces the laser intensity noise, but requires a great number of components such as additional laser diodes, a photodiode, and an optical coupler, resulting in higher costs and complexity of the total system. Electronic predistortion offers a simpler solution. By inserting an electronic predistorter in front of the laser to generate correcting signals, the overall linearity of the optical transmitter can be improved. A fully-integrated CMOS implementation of the laser predistortion circuit to improve the performance of the analog optical link offers a few advantages: 1) lower cost due to economies of scale since all the circuits will be on a single chip; 2) reduced power consumption; 3) smaller space requirement. In order to accommodate broadband wireless access into the optical network, large predistortion bandwidth is required. Due to the nonlinear nature of the predistortion circuit, the signal bandwidth, which is already broadband, expands to several bands at the predistorter's output. This necessitates an effective technique to extend the predistorter's output bandwidth. The semiconductor laser exhibits fluctuations in characteristics from component to component and changes in performance due to temperature change and aging effect. This necessitates adaptive calibration of the predistortion circuit in order to counteract the distortion variations among different laser diodes and correct the drift errors that would occur over time and temperature deviation for an individual laser diode.

\subsection{Thesis Objective}

The main objective of this research is to develop a fully integrated predistortion circuit, using standard CMOS technology for radio-over-fiber applications, and more generally, to 
develop a design methodology for predistortion circuit design in CMOS. For this purpose, a $2 \mathrm{GHz}$ center frequency is chosen for demonstration and a few specific design goals of this research are listed below:

- An effective technique is required to extend the predistorter's output bandwidth.

- Adjustable group delay is required for synchronizing the correction paths.

- On-chip tunability is required to account for laser variation.

- Broadband balun is required to connect the differential output of the predistortion circuit to the single-ended laser and hence to remove the even-mode distortion.

- Adaptive calibration technique is required for optimizing the predistorter's linearization performance.

To achieve these goals, the work to do includes studying and implementing the laser model for fast simulation, proposing new techniques to overcome design difficulties, prototype circuit design for fabrication, printed circuit board design and measurement setup.

\subsection{Thesis Contribution and Publications}

The main contribution in this thesis is the introduction of a new topology for a predistortion circuit. In this topology, multiple tank circuits are used to approximate the predistortion function only at the frequency range of interest, hence relieving the bandwidth requirement. A third order laser predistorter has been designed using CMOS 0.18 $\mu \mathrm{m}$ technology. A multiple variable feedback technique was also proposed to adaptively calibrate the predistortion circuit. Some other major contributions are listed below:

- Implemented behavioral model of the multi-tank predistorter and the semiconductor laser.

- Introduced a complete design procedure which defines each design step for the proposed multi-tank predistorter.

- Proposed and designed broadband baluns to reduce the even-mode distortion.

- Devised the calibration procedure. 
At the time of writing this thesis, the work described in the thesis resulted in four accepted publications [26-29]:

- Zhan Xu and Leonard MacEachern, "A Predistortion Circuit Design Technique for High Performance Analogue Optical Transmission," IEEE Micro-system and Nanoelectronics Research Conference, Oct. 2008

- Zhan Xu and Leonard MacEachern, "Multiple Stage Uncompensated Marchand Balun with 7:1 Bandwidth Ratio on Double Side PCB," Electronics Letters, May 2009

- Zhan Xu and Leonard MacEachern, "Optimum Design of Wideband Compensated and Uncompensated Marchand Baluns with Step Transformers," IEEE Transactions on Microwave Theory and Techniques, Aug. 2009

- Zhan Xu and Leonard MacEachern, "Adaptive Predistortion of Lasers Using Multiplevariable-feedback Algorithm," Electronics Letters, Sept. 2009

\subsection{Thesis Organization}

Chapter 1 introduces the background information and the objective of this research. Chapter 2 focuses on the analysis of the behavior of the laser. The rate-equation based model is discussed and analyzed in its circuit form. The laser model includes various nonlinearity effects and is implemented in the simulation environment. Based on this laser model, the pre-distorter model is derived by inverting the laser's current-power transfer function and introducing some time delay. Available laser linearization techniques are reviewed and design challenges for predistortion linearization are discussed in this chapter. Chapter 3 describes the proposed multi-tank technique, which is used to approximate the predistorter model. The analysis of mismatch effects and the optimization of circuit design parameters are described in this chapter. With all the design parameters having been derived, the circuit level implementation of the predistorter is described in Chapter 4. A design example is given in this chapter, then the design methodology is summarized. Chapter 5 describes the design and implementation of a broadband balun for canceling the even-mode distortions from the predistorter's output. Chapter 6 introduces an adaptive calibration 
technique, describes the measurement setup and demonstrates the measurement results. A summary of the entire thesis, comprising a list of contributions and recommendations for future work, is included in Chapter 7 . 


\section{Chapter 2}

\section{Laser Modeling and Linearization Techniques}

The ideal laser predistorter has a nonlinear transfer function which is the inverse of the laser's transfer characteristic. Therefore, by cascading a predistorter with the laser, an ideal linear transfer function, is produced. Hence, the optical output of the laser will faithfully follow the input signal without any distortion. In this chapter, the behavior of the semiconductor laser will be studied. The model for semiconductor lasers will be analyzed and inverted to derive the predistorter's transfer function. Available laser predistortion techniques will be reviewed and discussed in this chapter.

\subsection{Laser Principles and Laser Oscillation}

\subsubsection{Laser Principles}

LASER is an acronym for Light Amplification by the Stimulated Emission of Radiation. Ideal laser light is of a single wavelength, formed in parallel beams and it is coherent, that is, in a single phase. Laser operation involves three key light-matter interaction processes [30]:

- Photon absorbtion: when a photon of energy $\mathrm{h} \nu_{12}$ impinges on the system, an electron in state $E_{1}$ can absorb the photon energy and be excited to state $E_{2}$ as shown in Figure 2.1 a.

- Spontaneous emission: the electron at state $E_{2}$ will shortly return to the ground state without external stimulation as shown in Figure 2.1.b. 
- Stimulated emission: if a photon of energy $\mathrm{h} \nu_{12}$ impinges on the system while the electron is still in its excited state, the electron is immediately stimulated to drop to the ground state and give off a photon of energy $h \nu_{12}$ in phase with the incident photon as shown in Figure 2.1.c.

As shown in Figure 2.1 there are two energy states, ground state $E_{1}$ and the excited-state energy $E_{2}$. The transition between these two states involves the absorption or emission of photon of energy $h \nu_{12}=E_{2}-E_{1}$. In thermal equilibrium the density of excited electrons is very small. Most photons incident on the system will therefore be absorbed, so that stimulated emission is essentially negligible. Stimulated emission will exceed absorption only if the population of the excited states is greater than that of the ground state. This condition is known as population inversion. It is achieved by various pumping techniques. In semiconductor lasers, this is accomplished by the injection of electrons into the material at the device contacts to fill the lower energy states of the conduction band [31].

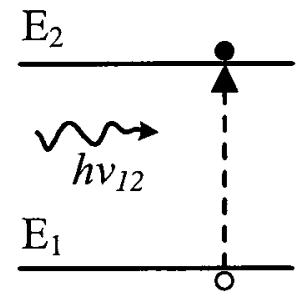

Absorption

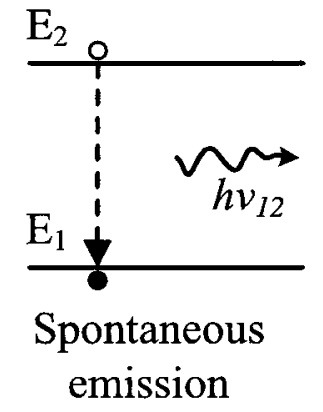

(b)

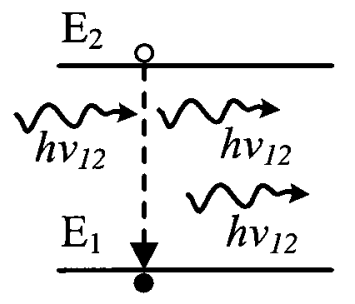

Stimulated emission

(c)

Figure 2.1: The three key processes in laser operation: absorption, spontaneous emission, and stimulated emission process [31].

\subsubsection{Explanation of Laser Oscillation}

Figure 2.2 gives an illustration of a Fabry-Perot type laser. As the light bounces back and forth in the active region, the light is amplified. The conditions for the wave to grow and finally oscillate are 


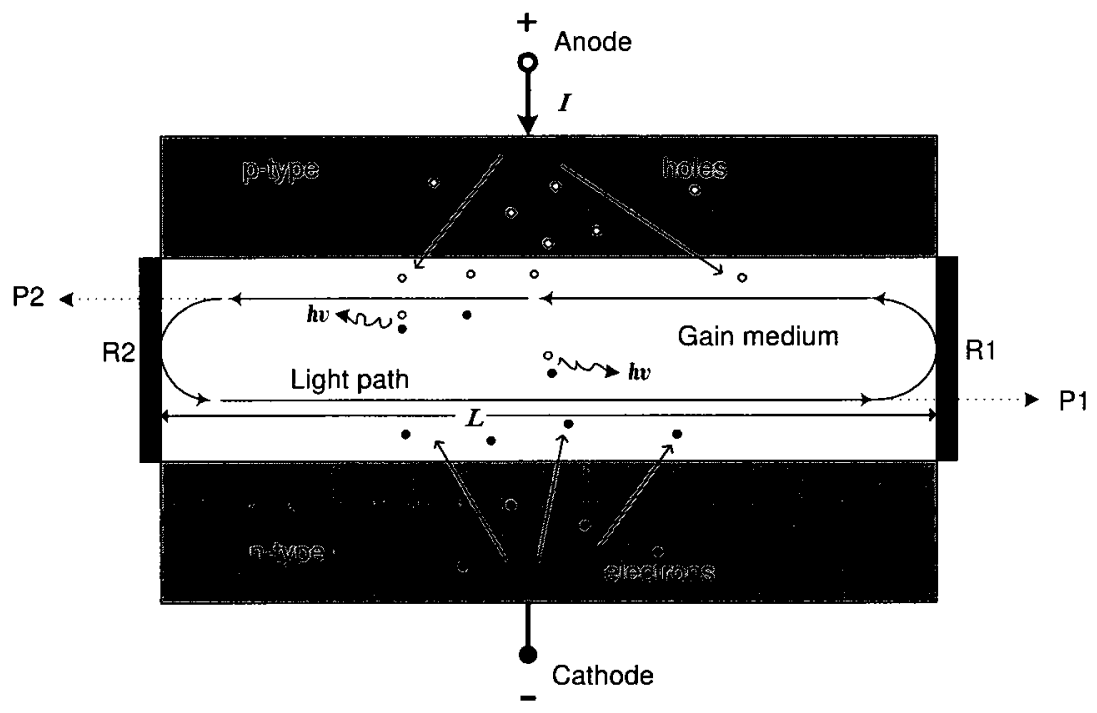

Figure 2.2: Schematic longitudinal view of a Fabry-Perot laser [31].

- The amplitude after each round trip has to be larger than or equal to the amplitude before the trip.

- The phase after each round trip has to be the same as the phase of the original trip. The light travels back and forth in the active region due to the reflection mirrors $\mathrm{R} 1$ and R2. Some power is lost because of the loss in the medium and photon escape from the mirror and the cavity. Taking these into account and writing in terms of complex amplitude (because of the wave nature of light), the amplification for one round trip is given by $[30,32]$

$$
E=E_{0} r_{1} r_{2} e^{\frac{1}{2}(g-\alpha) 2 L+j 2 \beta L}
$$

where $E_{0}$ is the amplitude of the light before the trip, $L$ is the length of the active region, $g$ is the net power gain due to stimulated emission and absorption, $\alpha$ is the power loss constant, and $\beta$ is the propagation constant. $r_{1}$ and $r_{2}$ are the reflection coefficients of the mirrors $R_{1}$ and $R_{2}$. The first condition is that the gain has to be larger than or equal to unity

$$
r_{1} r_{2} e^{\frac{1}{2}(g-\alpha) 2 L} \geqslant 1
$$


Then

$$
g \geqslant \frac{1}{L} \ln \frac{1}{r_{1} r_{2}}+\alpha
$$

The second condition is that the phase after each round trip has to be a integral multiple of $2 \pi$

$$
2 \beta L=2 n \pi
$$

where

$$
\beta=\frac{2 \pi \nu}{v_{g}}
$$

$v_{g}$ is the group velocity of the propagation mode supported by the longitudinal wave guide of the cavity and $\nu$ is the oscillation frequency of the propagation mode. Then the oscillation frequencies supported by the cavity are

$$
\nu=n \frac{v_{g}}{2 L}
$$

The net power gain $g$ includes the total result of stimulated emission and absorption. This net power gain depends on frequency $\nu$ and carrier density. An illustration of the gain spectrum shape is shown in Figure 2.3. The net power gain can be both negative and positive. The positive portion corresponds to population inversion, when the stimulated emission outnumbers the stimulated absorption. The power gain also depends on carrier density. The power gain increases with the increase of the carrier density as can be seen in the figures.

The bandpass shape of the gain spectrum curve is due to the carrier distribution in the energy bands of the semiconductor laser medium. If a p-type and an n-type semiconductor are joined, a nonequilibrium situation can be established. An illustration of the carrier distribution of a p-n junction is shown in Figure 2.4. A photon of energy $h \nu$ can stimulate emissions of photons of the same energy by inducing electrons to transit from the conduction band to empty states in the valence band. If a bias current is supplied to the p-n junction, the nonequilibrium distribution can be maintained. The shaded area in the conduction band are the energy states occupied by electrons, and the white area in the valence band are the states vacated by electrons, in other words, occupied by holes. 


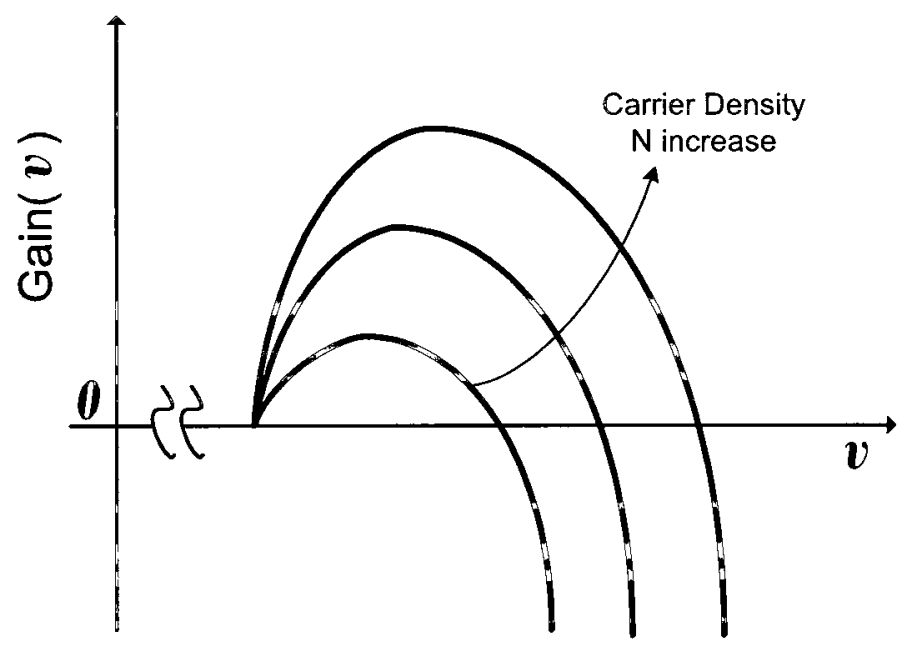

Figure 2.3: Gain spectrum.

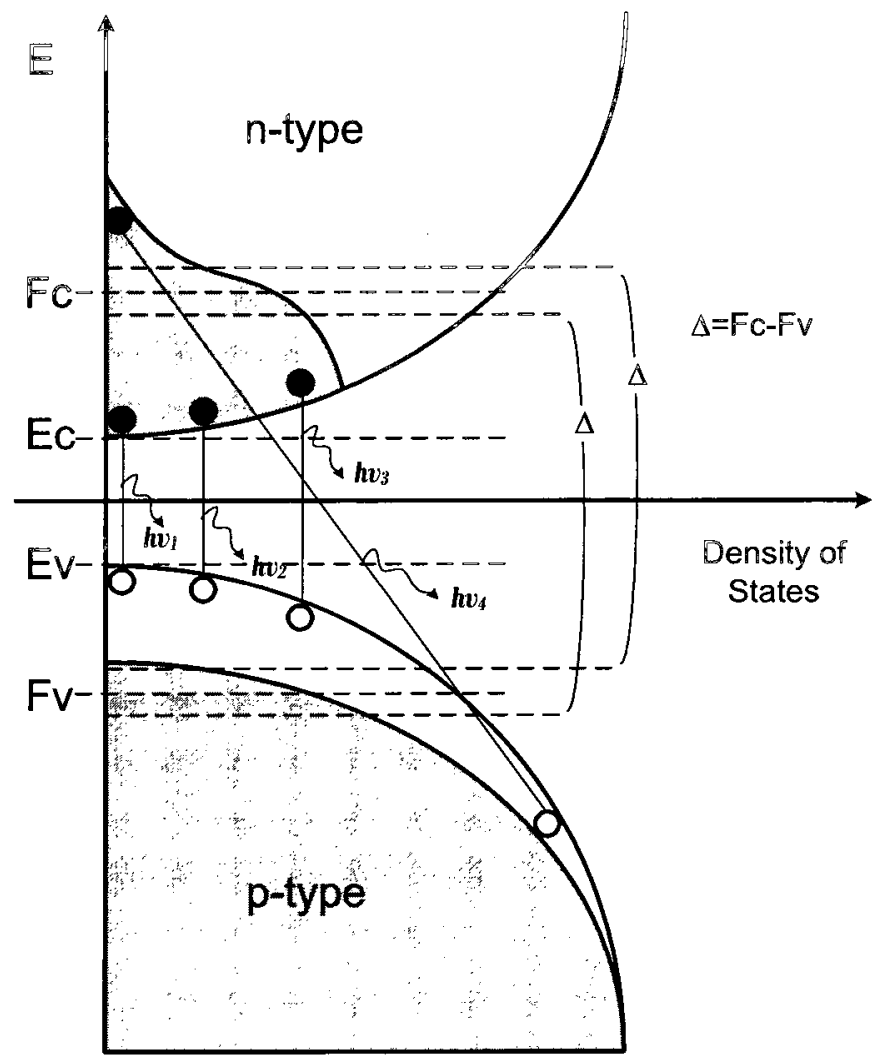

Figure 2.4: Illustration of the carrier distribution of a p-n junction [31]. 
As can be seen from the figure, the minimum photon energy due to stimulated emission is the band gap between the conduction band of n-type material and the valence band of the p-type material. So the lower limit for positive gain is

$$
h \nu>E_{c}-E_{v}
$$

The upper limit for positive gain is

$$
h \nu<F_{c}-F_{v}
$$

where $F_{c}$ and $F_{v}$ are the quasi-Fermi levels in the nonequilibrium situation. At these energy levels, the quantum states are half occupied. The probability of stimulated emission and absorption between the two energy states are equal. If we move a ruler up to measure two energy states with a difference of $F_{c}-F_{v}$, the probability of the stimulated emission is higher than the stimulated absorption between the two states. If we move a ruler down to measure two energy states with a difference of $F_{c}-F_{v}$, the probability of the stimulated absorption is higher than the stimulated emission. The probabilities of absorption and emission cancel each other out, the net gain is about zero for $h \nu=F_{c}-F_{v}$. For higher photon energy $h \nu$, higher energy difference between the two states is required. Because the number of electrons and holes decreases for higher energy difference, stimulated absorption becomes dominant, the net gain becomes negative for $h \nu>F_{c}-F_{v}$.

The gain peaks between the upper limit and lower limit, forming a flipped-bowl shape. This bowl-shaped spectrum selects the cavity's resonate frequencies to be amplified as illustrated in Figure 2.5. In a DFB type laser, a corrugated optical waveguide is used in the cavity. Compared to a Fabry-Perot type laser (where mirror reflection structures are used), the longitudinal modes supported by this corrugated periodic structure in a DFB type laser have a much wider frequency spacing between the adjacent modes. A DFB laser can be made such that only one longitudinal mode falls into the bowl-shaped gain spectrum to get amplified. Hence the laser can operate in a single longitudinal mode. 

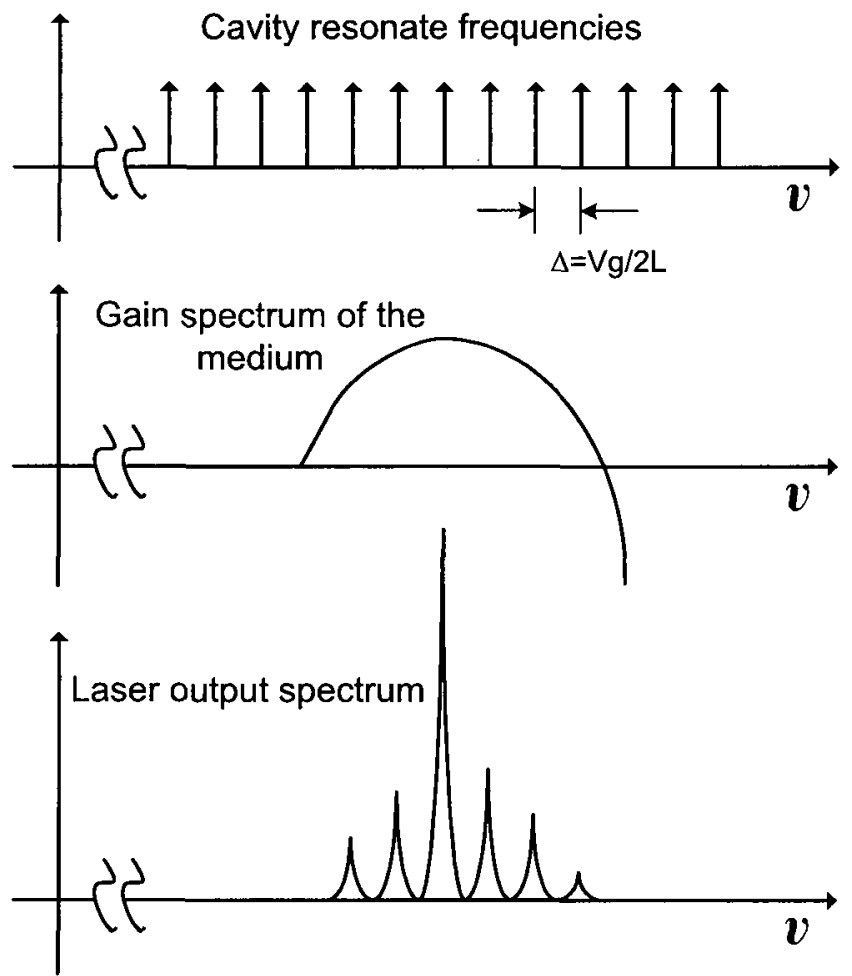

Figure 2.5: Laser output spectrum. 


\subsection{Laser Model}

\subsubsection{Rate Equations}

Semiconductor lasers are commonly modeled by rate equations for simulating the dynamics of the electron and photon population $[30,33,34]$. The conventional laser rate equations can be expressed as

$$
\begin{aligned}
\frac{d C(t)}{d t} & =\frac{I(t)}{q V_{c}}-\frac{C(t)}{\tau_{e}}-G P(t) \\
\frac{d P(t)}{d t} & =\Gamma \beta_{s p} \frac{C(t)}{\tau_{e}}-\frac{P(t)}{\tau_{p h}}+\Gamma G P(t) .
\end{aligned}
$$

More specifically, the rate equations describe the change rate of electron density and photon density in the active region with respect to time. With the initial condition

$$
\begin{aligned}
& P(0)=0 \\
& C(0)=0 \\
& I(0)=0
\end{aligned}
$$

a large signal input-output relationship between the injection current and the photon density

$$
P(t)=f[I(t)]
$$

can be obtained. In the above equations, $P$ is the active region photon density, $C$ is the active region carrier (electron) density. $\Gamma$ is the mode confinement factor, which measures the mode power confined within the active region relative to the total mode power. $\beta_{s p}$ is the spontaneous emission factor, which is the factor to measure the spontaneous emission whose spectrum overlaps with that of the stimulated emission. $\tau_{e}$ is the electron life time. $\tau_{p h}$ is the photon life time. $P / \tau_{p h}$ describes the number of photons diminished due to either absorption or escape from unit volume and the mirrors per unit time. $I$ is the injection current, $q$ is the electronic charge, $G$ is the optical gain for a specific longitudinal mode (frequency). $V_{c}$ is the volume of the active region. The photon density $P$ is related to the output optical power by:

$$
P_{o u t}^{o p t}=\frac{1}{2} \frac{h \nu P V_{c}}{\tau_{e s}}
$$


where $h$ is the Plank constant, $\nu$ the frequency and $\tau_{e s}$ the photon escape time from the active region.

The optical gain $G$ is the value on the gain spectrum curve for the stimulated emission mode (frequency). This gain is related to both carrier density and optical density. There are several phenomena which cause the optical gain to saturate. Two main phenomena are spatial and spectral hole burning. Spatial hole burning occurs as a result of the standing wave nature of the optical modes. Increased lasing power results in decreased carrier diffusion efficiency which means that the stimulated recombination time becomes shorter relative to the carrier diffusion time. Carriers are therefore depleted faster at the crest of the wave causing a decrease in the modal gain. Spectral hole burning is related to the gain profile broadening mechanisms such as short intra-band scattering which is related to power density.

To account for gain saturation due to the high power densities in semiconductor lasers, the gain equation is modified such that it becomes related to the inverse of the optical power. The expression for gain can be formulated as [35]

$$
G=\frac{v_{g} \cdot g \cdot\left(C-C_{t r}\right)}{\sqrt{1+\delta \cdot P}}
$$

where $\sqrt{1+\delta \cdot P}^{-1}$ is the gain saturation term and $\delta$ is the compression factor. $v_{g}$ is the group velocity. $g$ is the gain coefficient. $C-C_{t r}$ is the first order approximation of the effect of carrier density. $C_{t r}$ is the transparency carrier density, which is the carrier density corresponding to the onset of population inversion. More specifically, $C_{t r}$ corresponds to the state that the stimulated emission is equal to the stimulated absorption, hence the maximum of the gain curve is at zero.

\subsubsection{Rate Equations Based Model in ADS}

Different methods for the laser model implementation have been discussed in [36]. In this thesis, Agilent-ADS (Agilent Advanced Design System) was chosen for the implementation of the laser model by following a similar procedure described in [35]. ADS has both a circuit simulator and EM simulation tools and it allows devices defined by equations, which makes 
it possible to do a co-simulation including transistors, inductors and equation-based lasers. ADS offers both time domain and large signal frequency domain techniques. It is convenient to characterize a circuit's large signal behavior in both time domain and frequency domain in ADS.

The input current $I(t)$ is in the order of $10^{-3} \mathrm{~A}$ and the carrier density $C(t)$ and photon density $P(t)$ are in the order of $10^{24}$. A very high accuracy on the simulation convergence is required with these widely-spread numerical values. This accuracy is usually beyond a circuit simulator's capability whose convergence relative tolerance is usually $10^{-6}$ and absolute tolerance is usually $10^{-12}$. Considering that circuit simulators are optimized for circuit components (such as capacitors in the range of $\mathrm{pF}-\mathrm{nF}$ ) and the laser input current is in $\mathrm{mA}$ and laser output is in $\mathrm{mW}$, the electron density $C(t)$ and photon density $P(t)$ need to be scaled down to these ranges.

Considering equation (2.2.7) which converts photon density to laser output power, it would be practical to scale down $P(t)$ by defining

$$
P^{n}=P_{\text {out }}=P \cdot \frac{h \nu V_{c}}{2 \tau_{e s}}
$$

and normalized compression factor

$$
\delta^{n}=\delta \cdot \frac{2 \tau_{e s}}{h \nu V_{c}}
$$

Considering equation (2.3.12) which converts threshold electron density to threshold current and neglecting $\beta_{s p}$, it would be practical to scale down $C(t)$ by defining

$$
C^{n}=C \cdot \frac{q V_{c}}{\tau_{e}}
$$

and

$$
C_{t r}^{n}=C_{t r} \cdot \frac{q V_{c}}{\tau_{e}}
$$

Substituting them into rate equations (2.2.1) and (2.2.2), after rearranging, it can be obtained that

$$
C_{1} \frac{d C_{(t)}^{n}}{d t}=I(t)-C_{(t)}^{n}-A_{g}\left(C_{(t)}^{n}-C_{t r}^{n}\right)\left(1+\delta^{n} P_{(t)}^{n}\right)^{-1 / 2} P_{(t)}^{n}
$$




$$
C_{2} \frac{d P_{(t)}^{n}}{d t}=\beta_{s p} C_{(t)}^{n}-G_{2} P_{(t)}^{n}+A_{g}\left(C_{(t)}^{n}-C_{t r}^{n}\right)\left(1+\delta^{n} P_{(t)}^{n}\right)^{-1 / 2} P_{(t)}^{n}
$$

where

$$
\begin{gathered}
C_{1}=\tau_{e}, \\
C_{2}=\frac{2 \tau_{e s} q}{\Gamma h \nu}, \\
G_{2}=\frac{2 \tau_{e s} q}{\Gamma h \nu \tau_{p h}},
\end{gathered}
$$

and

$$
A_{g}=\frac{2 \tau_{e} v_{g} g \tau_{e s}}{h \nu V_{c}}
$$

It has been demonstrated in [37] that the laser rate equations have two sets of solutions. In order for the simulator to find the correct solution which gives the positive optical power, a quadratic transformation has been suggested in [37] and redefined here as

$$
P^{n}=\left(P_{s q r t}^{n}+d y\right)^{2}
$$

$d y$ is a negligible small number (for example, $10^{-10}$ was used in this thesis), which is used to avoid the divided-by-zero error [37].

Table 2.1: Parameters values for a 1.3 $\mu \mathrm{m}$ InGaAsP/InP Fujitsu FLD3F7CZ semiconductor laser [35].

\begin{tabular}{|c|c|c|}
\hline Parameter & Symbol, Unit & Value \\
\hline \hline Operating wave length & $\lambda, \mu m$ & 1.3 \\
\hline Active region volume & $V_{c}, m^{3}$ & $24 \times 10^{-18}$ \\
\hline Transparency carrier density & $C_{t r o}, m^{-3}$ & $1.0 \times 10^{24}$ \\
\hline Mode confinement factor & $\Gamma$ & 0.16 \\
\hline Gain coefficient & $v_{g} \cdot g, m^{3} s^{-1}$ & $3.04 \times 10^{-12}$ \\
\hline Spontaneous emission factor & $\beta_{s p}$ & $5 \times 10^{-5}$ \\
\hline Photon life time & $\tau_{p h}$ & $1.0 \times 10^{-12}$ \\
\hline Electrons life time & $\tau_{e}$ & $1.5 \times 10^{-9}$ \\
\hline Gain compression & $\delta, m^{-3}$ & $2.3 \times 10^{-23}$ \\
\hline Photon Escaping time & $\tau_{e s}$ & $1.33 \times 10^{-12}$ \\
\hline Saturation Current & $I_{s}, \mathrm{~A}$ & $3 \times 10^{-10}$ \\
\hline Junction Ideality factor & $\eta$ & 2 \\
\hline
\end{tabular}




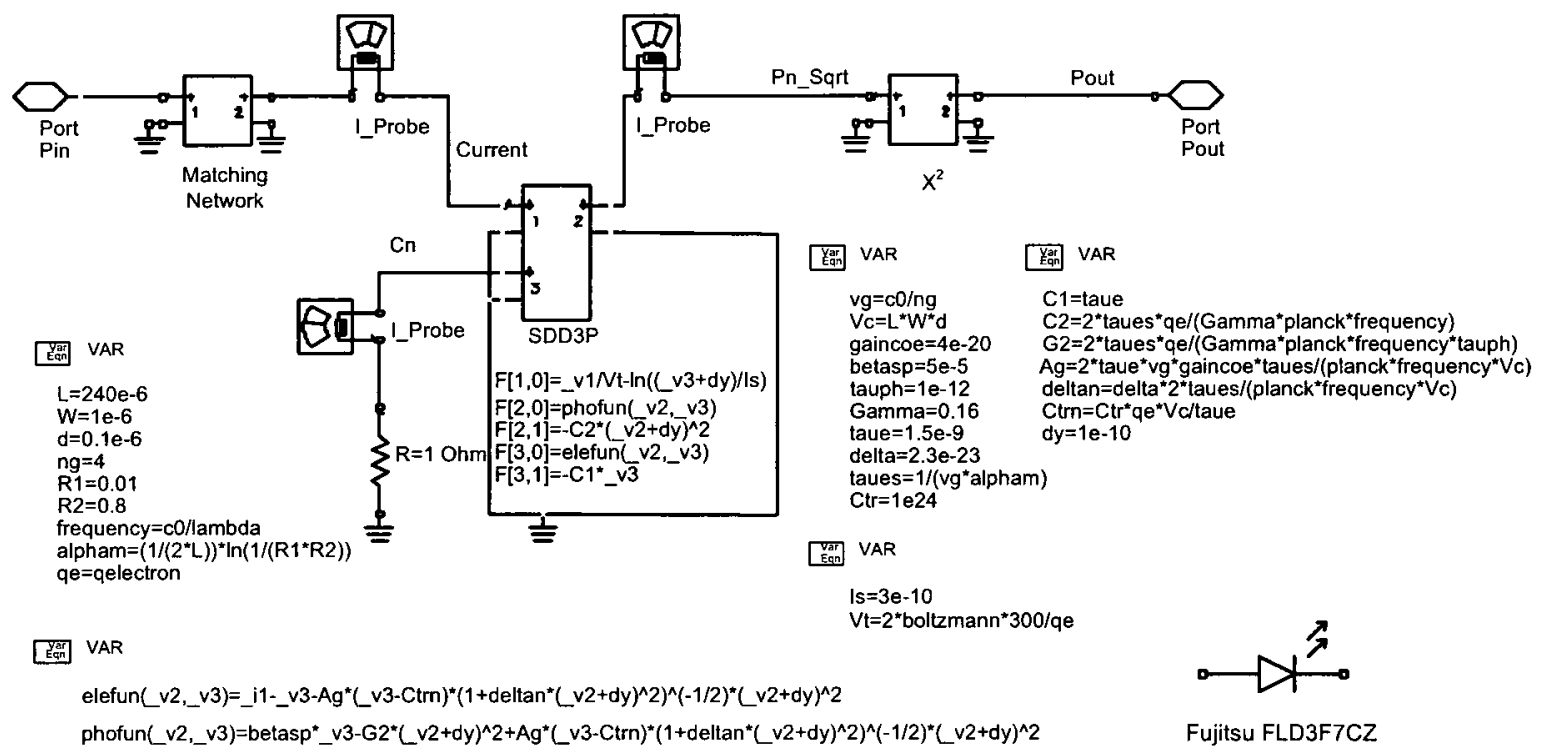

Figure 2.6: Symbolic laser model defined in ADS.

Table 2.1 lists the extracted parameters of a $1.3 \mathrm{um}$ InGaAsP/InP Fujistu FLD3F7CZ semiconductor laser [35]. The laser diode model implemented in ADS, using the equationsbased symbolic device, is shown in Figure 2.6. The port voltage $v_{2}$ represents the normalized electron density $C^{n}$ and $v_{3}$ is the square root of the normalized photon density $P^{n}$. The $X^{2}$ component converts $v_{3}$ to the laser output in a voltage form using Volt for Watt. The matching network stores the s-parameter file for the package parasitics. To be discussed in section 2.3.1, the carrier density is almost constant, and the laser is biased close to the threshold voltage. Hence, the diode junction voltage can be modeled by a simple Shockley diode, where the diode spontaneous recombination current is given by

$$
I_{s p}=\frac{q V_{c} C}{\tau_{e}} \approx I_{s} e^{\frac{q V_{j}}{\eta k T}}
$$

where $I_{s}$ is the junction saturation current and $\eta$ is the junction ideality factor. Therefore,

$$
V_{j}=\frac{\eta k T}{q} \ln \left(\frac{C^{n}}{I_{s}}\right)
$$

which is defined as $v_{1}$ in the symbolic laser model. 


\subsection{Analysis of Laser Diode Behavior}

For the ease of analyzing the laser behavior, the laser rate equations (2.2.13) and (2.2.14) are expressed in the circuit form shown in Figure 2.7:

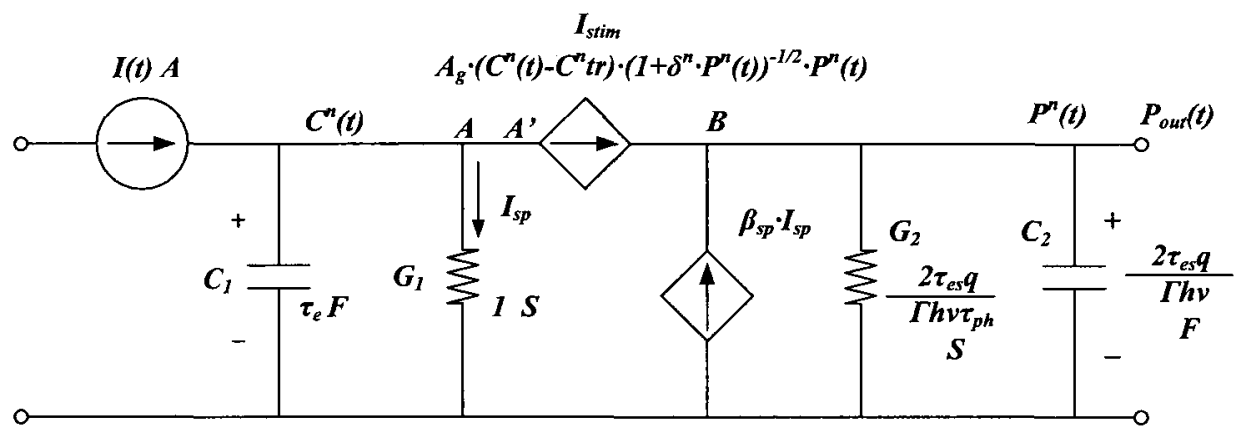

Figure 2.7: Equivalent circuit of the laser rate equations.

\subsubsection{Analysis}

When the laser is driven by dc signal (bias current), the laser rate equations can be simplified to the steady-state rate equations

$$
0=\Gamma \beta_{s p} \frac{C_{d c}}{\tau_{e}}-\frac{P_{d c}}{\tau_{p h}}+\Gamma G P_{d c}
$$

and

$$
0=\frac{I_{d c}}{q V_{c}}-\frac{C_{d c}}{\tau_{e}}-G P_{d c}
$$

Considering the nonlinear gain compression term, obtaining the I-P relation in a analytical form is difficult and a numerical technique has to be used. However, through circuit analysis, a simple solution with sufficient accuracy can be obtained. In the circuit form of the laser model (Figure 2.7), $I_{\text {stim }}$ represents positive feedback at node B, which can be expressed as a negative conductance in the form of

$$
G_{n}=-A_{g}\left(C_{d c}-C_{t r}\right)(1+\delta P)^{-1 / 2}
$$

$I_{\text {stim }}$ also provides negative feedback at node A by drawing current from node A, therefore maintaining a steady output. Hence,

$$
0>G_{n}>-G_{2}
$$


must be satisfied for the steady output. With (2.3.3), (2.2.17) and (2.2.18), neglecting the gain compression term, it can be obtained that

$$
C_{t r}<C_{d c}<C_{t h}=C_{t r}+\frac{1}{\Gamma \tau_{p h} g \nu_{g}}
$$

Considering that

$$
P_{\text {out }}=\frac{\beta_{s p} I_{s p}}{G_{2}+G_{n}}
$$

and the $\beta_{s p} I_{s p}$ term corresponds to the spontaneous emission fallen into the spectrum of the stimulated emission, which is usually very small, hence

$$
G_{n} \approx-G_{2}
$$

Therefore,

$$
\begin{gathered}
C_{d c} \approx C_{t h} \\
C_{d c}^{n} \approx C_{t h} \cdot \frac{q V_{c}}{\tau_{e}} \\
I_{s p} \approx C_{t h} \cdot \frac{q V_{c}}{\tau_{e}} .
\end{gathered}
$$

At last,

$$
P_{d c}=\frac{I_{d c}-I_{t h}}{G_{2}}
$$

where

$$
I_{t h}=\left(1-\beta_{s p}\right) \cdot C_{t h} \cdot \frac{q V_{c}}{\tau_{e}} .
$$

For the condition that $I_{d c}$ is less than $I_{t h}$,

$$
P_{d c} \approx 0
$$

With the parameters defined in the Table 2.1 , the threshold current can be obtained as

$$
I_{t h}=7.9 m A
$$

and the slope can be obtained as

$$
\frac{d P_{d c}}{d I_{d c}}=\frac{1}{G_{2}}=0.05752 .
$$

The simulated dc response is shown in Figure 2.8. The calculated slope and $I_{t h}$ agree well with the simulation results. 


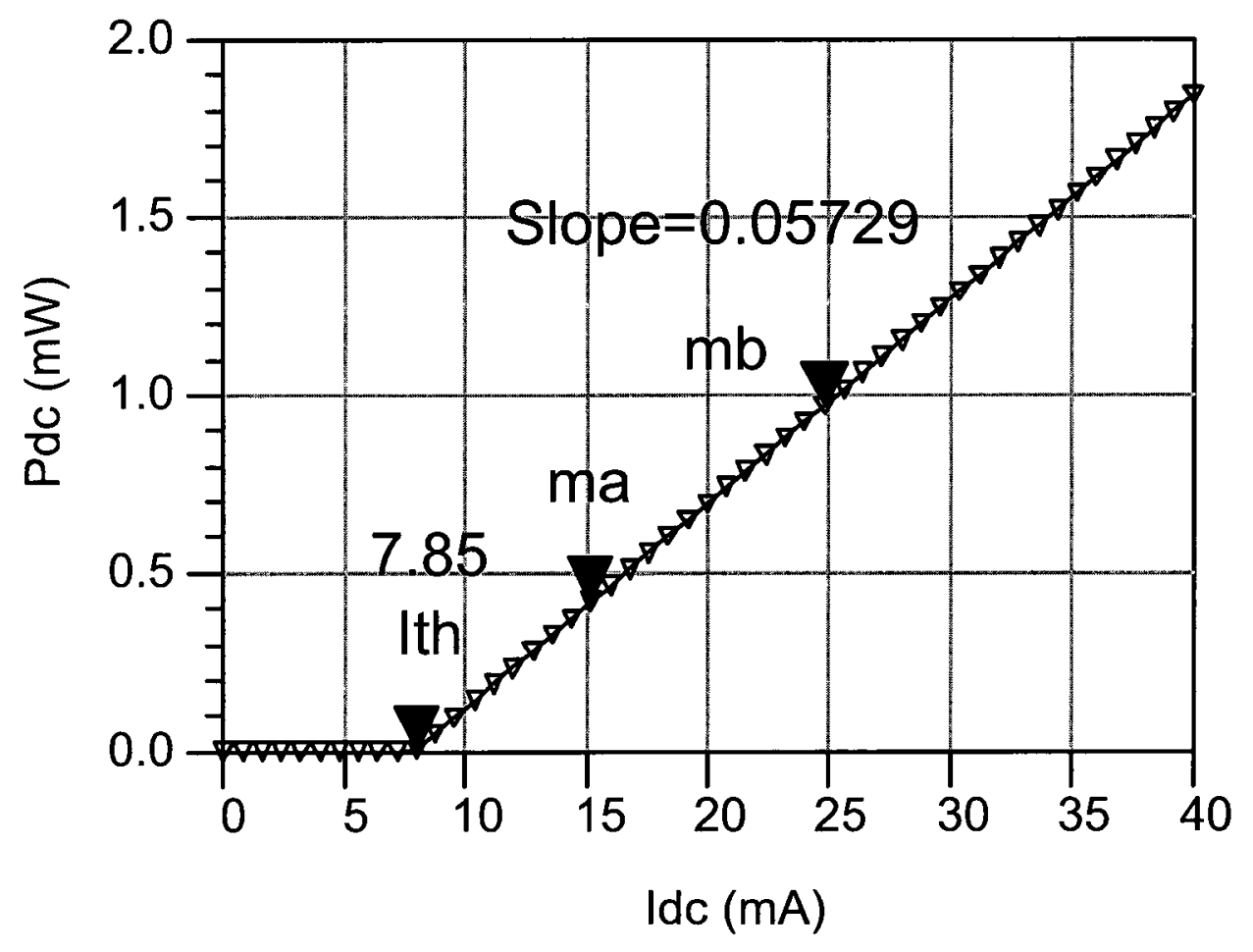

Figure 2.8: Laser model dc response. 


\subsubsection{Small Signal Analysis}

For the small signal intensity modulation, the equivalent circuit of Figure 2.7 can be simplified by linearizing the nonlinear component $I_{\text {stim }}$ at the dc operating point. The linearized equivalent circuit is depicted in Figure 2.9. The small signal parameters of this circuit are obtained by taking partial derivatives of $I_{\text {stim }}$ with respect to $c^{n}(t)$ and $p^{n}(t)$ and neglecting the gain compress term:

$$
\begin{gathered}
G_{c}=\left.\frac{\partial I_{s t i m}}{\partial c^{n}(t)}\right|_{@ P_{d c}^{n}}=A_{g} \cdot P_{d c}^{n} \\
g m_{c}=\left.\frac{\partial I_{s t i m}}{\partial p^{n}(t)}\right|_{@ C_{d c}^{n}}=A_{g} \cdot\left(C_{d c}^{n}-C_{t r}^{n}\right) \\
G_{p}=\left.\frac{\partial I_{s t i m}}{\partial p^{n}(t)}\right|_{@ C_{d c}^{n}}=-A_{g} \cdot\left(C_{d c}^{n}-C_{t r}^{n}\right) \\
g m_{p}=\left.\frac{\partial I_{s t i m}}{\partial c^{n}(t)}\right|_{@ P_{d c}^{n}}=A_{g} \cdot P_{d c}^{n}
\end{gathered}
$$

where $P_{d c}^{n}$ can be obtained from (2.3.11) and $g m_{c}$ can be obtained from (2.3.10) as

$$
g m_{c}=A_{g} \cdot\left(C_{d c}^{n}-C_{t r}^{n}\right)=\frac{I_{s t i m}}{P_{d c}^{n}}=\frac{I_{d c}-C_{t h} q V_{c} / \tau_{e}}{P_{d c}^{n}} .
$$

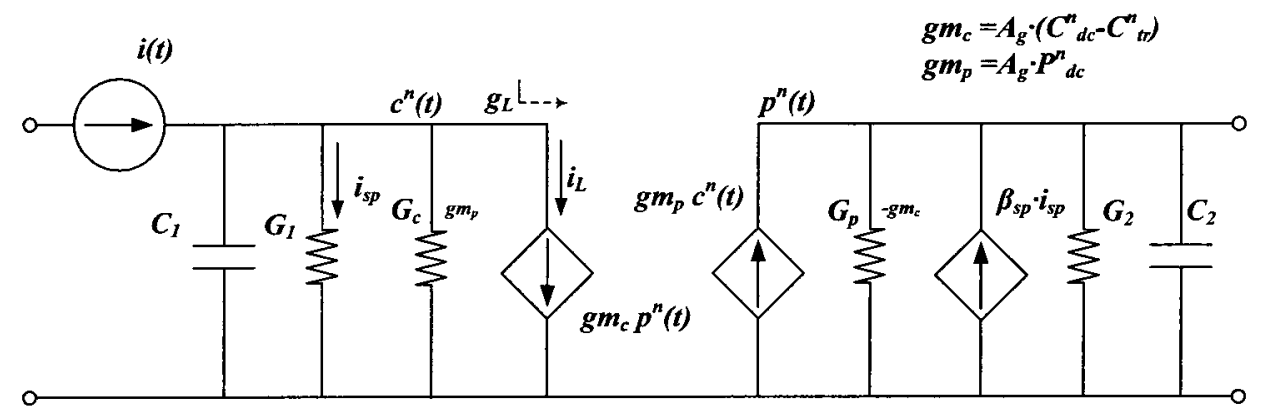

Figure 2.9: Small signal equivalent circuit of the laser rate equations.

The impedance looking toward the photon emission side can be found as

$$
\begin{aligned}
g_{L} & =\frac{g m_{c} p^{n}(s)}{c^{n}(s)} \\
& =\frac{g m_{c} \frac{g m_{p} c^{n}(s)+\beta_{s p} c^{n}(s)}{G_{2}-g m_{c}+s C_{2}}}{c^{n}(s)}
\end{aligned}
$$




$$
=\frac{g m_{c}\left(g m_{p}+\beta_{s p}\right)}{G_{2}-g m_{c}+s C_{2}}
$$

and

$$
i_{L}=i(s) \frac{g_{L}}{g_{L}+g m_{p}+G_{1}+s C_{1}} .
$$

The laser output can be obtained as

$$
p^{n}(s)=\frac{i_{L}}{g m_{c}} .
$$

At last, the transfer function can be found as:

$$
\frac{p^{n}(s)}{i(s)}=\frac{\left(\beta_{s p}+g m_{p}\right) /\left(C_{1} C_{2}\right)}{\frac{\beta_{s p} g m_{c}+G_{2} g m_{p}-G_{1} g m_{c}+G_{1} G_{2}}{C_{1} C_{2}}+\left(\frac{G_{1}+g m_{p}}{C_{1}}+\frac{G_{2}-g m_{c}}{C_{2}}\right) s+s^{2}} .
$$

It is a second order low pass response with the relaxation oscillation frequency at

$$
\begin{aligned}
\omega_{0} & =\sqrt{\frac{\beta_{s p} g m_{c}+G_{2} g m_{p}-G_{1} g m_{c}+G_{1} G_{2}}{C_{1} C_{2}}} \approx \sqrt{\frac{G_{2} g m_{p}}{C_{1} C_{2}}} \\
& =\sqrt{\frac{\Gamma v_{g} g\left(I_{d c}-I_{t h}\right)}{q V_{c}}} .
\end{aligned}
$$

With the parameters defined in the Table 2.1, the relaxation oscillation frequency for 30 $\mathrm{mA}$ bias current can be obtained as

$$
f_{0}=\frac{\omega_{0}}{2 \pi}=8.35 G H z
$$

The simulated ac response is shown in Figure 2.10. The peak of each curve corresponds to the relaxation oscillation frequency. It is demonstrated that increasing the laser bias current moves the relaxation oscillation to a higher frequency.

\subsubsection{Large Signal Nonlinearity}

Laser nonlinearity mostly comes from two mechanisms, optical gain saturation and nonlinear coupling between the stimulated photons and the injected electrons. There are many mechanisms cause the gain saturation, such as spatial hole-burning [38], carrier 


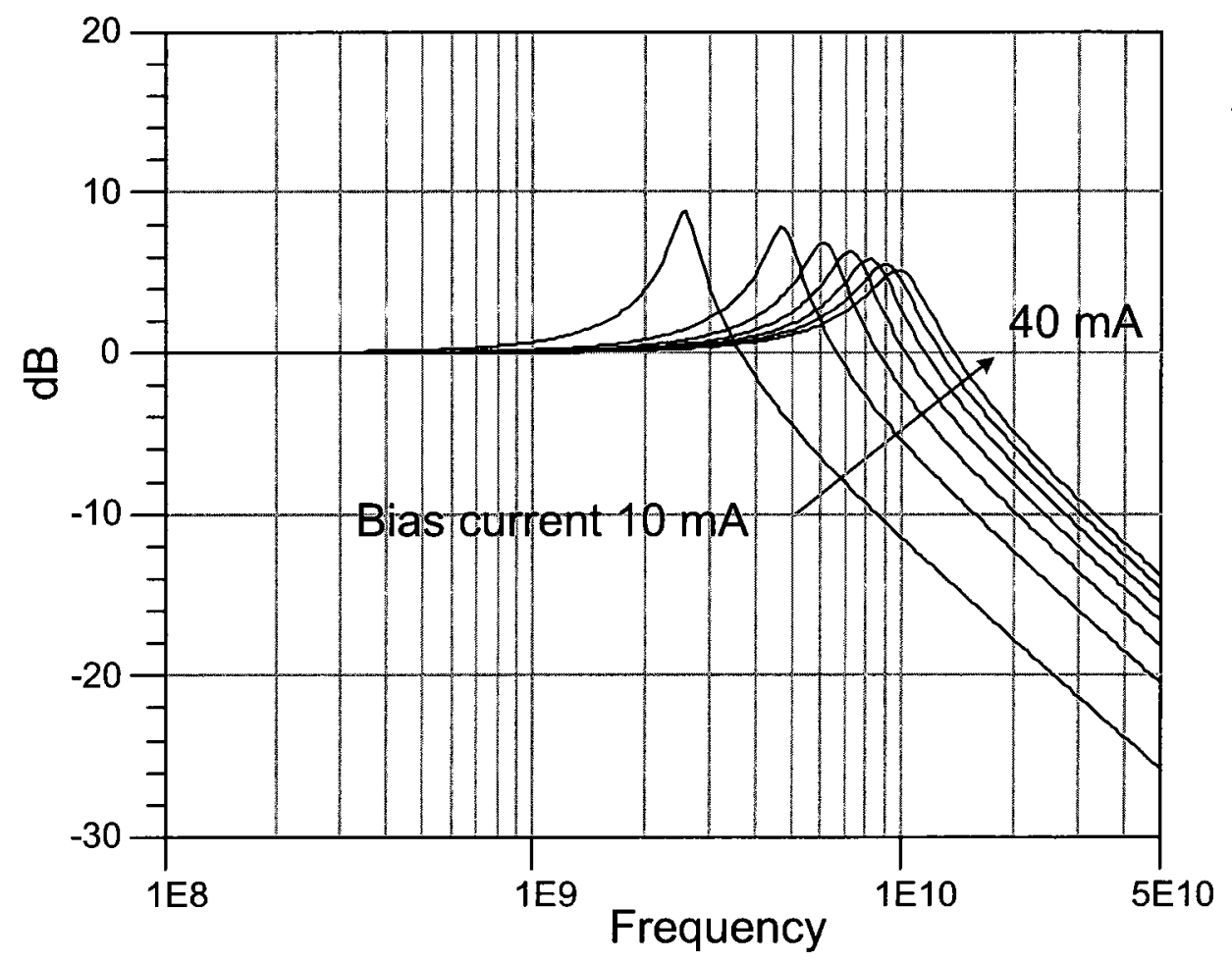

Figure 2.10: Laser model ac response. 
heating [39-41] and current leakage [41,42]. The gain saturation are usually included by adopting a power-dependant gain-suppression term in the following forms:

$$
\begin{array}{ll}
a: & (1-\delta \cdot P) \\
b: & (1+\delta \cdot P)^{-1} \\
c: & (1+\delta \cdot P)^{-1 / 2} .
\end{array}
$$

Form $\mathrm{c}$ is obtained by using density matrix approach to include intraband transitions effects. It has been demonstrated in [35] that gain suppression of form c provides better agreement between the simulated and measured results. The expression for modal gain is written as :

$$
G=\frac{v_{g} \cdot g \cdot\left(C-C_{t r}\right)}{\sqrt{1+\delta \cdot P}}
$$

where $\delta$ is the compression factor.

The mechanism of nonlinear coupling between photons and electrons is represented by the negative feedback current $I_{\text {stim }}$ shown in Figure 2.7 and the reprinted Figure 2.11 below. It can be readily seen that this negative feedback works nonlinearly, as the feedback current is a multiple of functions of photon and electron density.

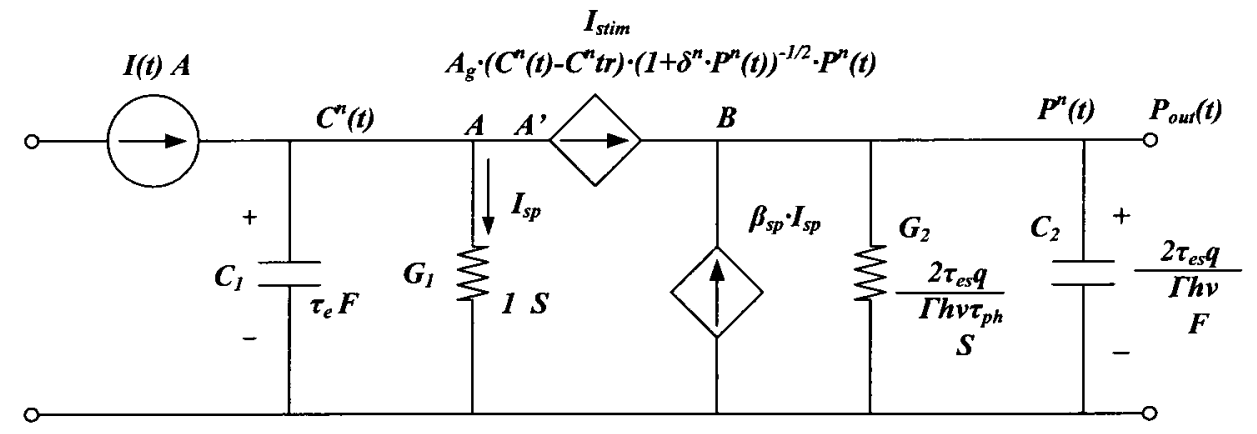

Figure 2.11: Equivalent circuit of the laser rate equations.

The nonlinear component $I_{\text {stim }}$ represents the interaction between electrons and photons. Neglecting gain compression, it can be represented at the dc operating point by:

$$
\begin{aligned}
I_{s t i m} & =A_{g}\left(C_{d c}^{n}+c-C_{t r}^{n}\right)\left(P_{d c}^{n}+p\right) \\
& =A_{g}\left(C_{d c}^{n}-C_{t r}^{n}\right) p+A_{g} P_{d c}^{n} c+A_{g} c p+A_{g}\left(C_{d c}^{n}-C_{t r}^{n}\right) P_{d c}^{n}
\end{aligned}
$$


The first and second term are linear components, the third term is the cross multiplication components and the last term is the dc operating point. The nonlinear responses around the dc operating point of this circuit can be described by Volterra transfer functions. The first order Volterra transfer functions can be computed using the linearized circuit shown in Figure 2.9 and the reprinted Figure 2.12. Applying KCL at node 1 and 2, the circuit

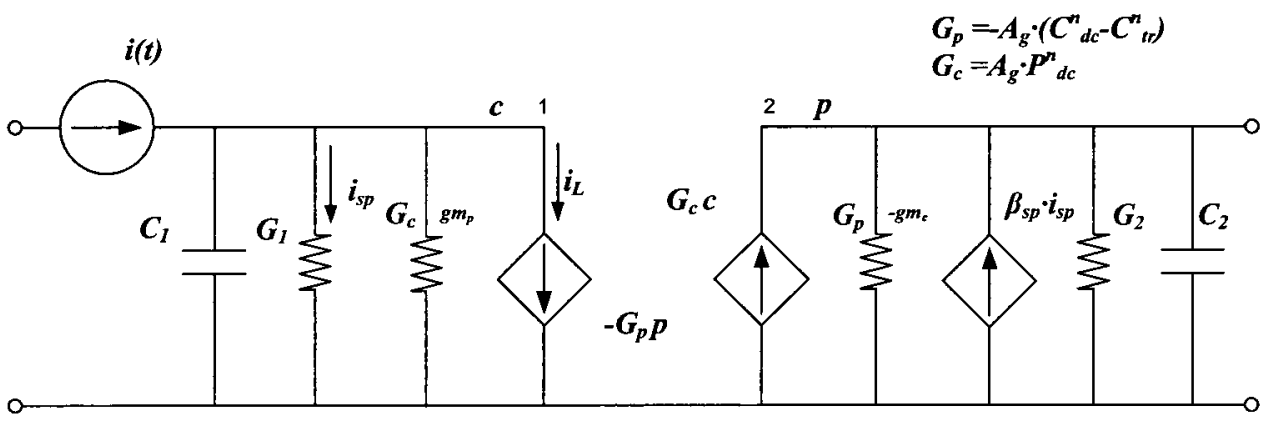

Figure 2.12: Linearized circuit for calculating first order Volterra kernel.

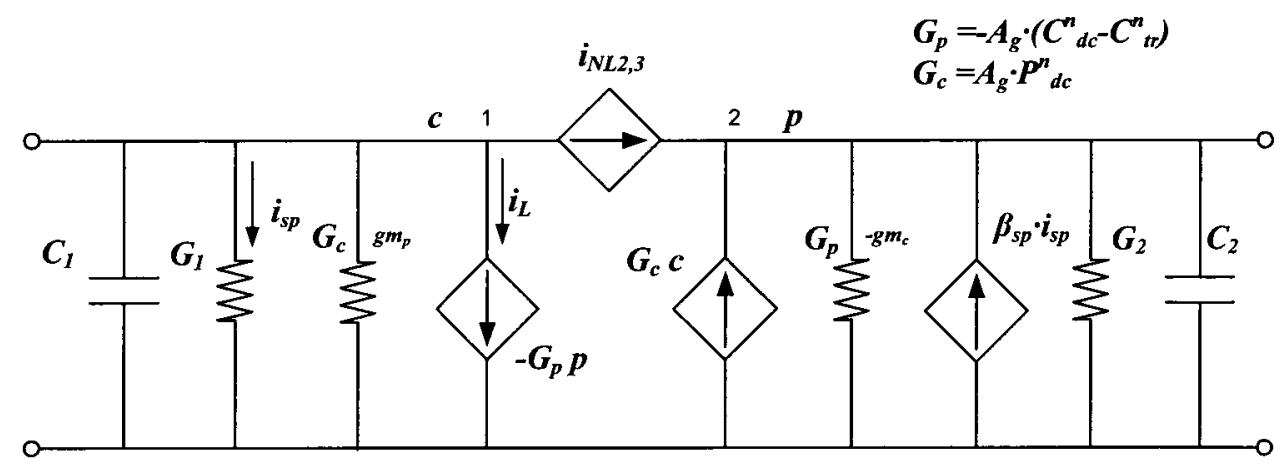

Figure 2.13: Equivalent circuit for calculating second or third order Volterra kernel.

matrix equation can be obtained as:

$$
\left|\begin{array}{cc}
s C_{1}+G_{1}+G_{c} & -G_{p} \\
-G_{1} \beta_{s p}-G_{c} & s C_{2}+G_{2}+G_{p}
\end{array}\right| \cdot\left|\begin{array}{c}
H_{11}(s) \\
H_{12}(s)
\end{array}\right|=\left|\begin{array}{c}
1 \\
0
\end{array}\right|
$$

By solving the equations, the first order Volterra kernels can be obtained as:

$$
H_{11}=\frac{s C_{2}+G_{2}+G_{p}}{s^{2} C_{1} C_{2}+s\left(C_{2} G_{1}+C_{1} G_{2}+C_{2} G_{c}+C_{1} G_{p}\right)+\left(G_{1} G_{p}+G_{1} G_{2}+G_{2} G_{c}-G_{1} G_{p} \beta_{s p}\right)}
$$




$$
H_{12}=\frac{G_{c}+G_{1} \beta_{s p}}{s^{2} C_{1} C_{2}+s\left(C_{2} G_{1}+C_{1} G_{2}+C_{2} G_{c}+C_{1} G_{p}\right)+\left(G_{1} G_{p}+G_{1} G_{2}+G_{2} G_{c}-G_{1} G_{p} \beta_{s p}\right)}
$$

Using the standard procedure described in [43], the second and third order Volterra transfer functions can be computed using the equivalent circuit shown in Figure 2.13, where the external signal sources are removed and second-order or third-order nonlinear current sources are applied for correcting the second- and third-order nonlinearity in the circuit. Applying KCL, the circuit equations for the second order Volterra kernels can be obtained as:

$$
\left|\begin{array}{cc}
\left(s_{1}+s_{2}\right) C_{1}+G_{1}+G_{c} & -G_{p} \\
-G_{1} \beta_{s p}-G_{c} & \left(s_{1}+s_{2}\right) C_{2}+G_{2}+G_{p}
\end{array}\right| \cdot\left|\begin{array}{c}
H_{21}\left(s_{1}, s_{2}\right) \\
H_{22}\left(s_{1}, s_{2}\right)
\end{array}\right|=\left|\begin{array}{c}
-i_{N L 2} \\
i_{N L 2}
\end{array}\right|
$$

where $i_{N L 2}$ is the second order nonlinear current source for the two-dimensional conductance $I_{\text {stim }}$

$$
i_{N L 2}=\frac{A_{g}}{2}\left(H_{11}\left(s_{1}\right) H_{12}\left(s_{2}\right)+H_{11}\left(s_{2}\right) H_{12}\left(s_{1}\right)\right) .
$$

By solving the equations, the second order Volterra kernels can be obtained as:

$$
\begin{aligned}
& H_{21}=\frac{-i_{N L 2}\left(G_{2}+s C_{2}\right)}{s^{2} C_{1} C_{2}++s\left(C_{2} G_{1}+C_{1} G_{2}+C_{2} G_{c}+C_{1} G_{p}\right)+\left(G_{1} G_{p}+G_{1} G_{2}+G_{2} G_{c}-G_{1} G_{p} \beta_{s p}\right)} \\
& H_{22}=\frac{i_{N L 2}\left(G_{1}-G_{1} \beta_{s p}+s C_{1}\right)}{s^{2} C_{1} C_{2}++s\left(C_{2} G_{1}+C_{1} G_{2}+C_{2} G_{c}+C_{1} G_{p}\right)+\left(G_{1} G_{p}+G_{1} G_{2}+G_{2} G_{c}-G_{1} G_{p} \beta_{s p}\right)}
\end{aligned}
$$

where

$$
s=s_{1}+s_{2}
$$

Similarly, the circuit equations for the third order Volterra kernels can be obtained as:

$$
\left|\begin{array}{cc}
\left(s_{1}+s_{2}+s_{3}\right) C_{1}+G_{1}+G_{c} & -G_{p} \\
-G_{1} \beta_{s p}-G_{c} & \left(s_{1}+s_{2}+s_{3}\right) C_{2}+G_{2}+G_{p}
\end{array}\right| \cdot\left|\begin{array}{c}
H_{31}\left(s_{1}, s_{2}, s_{3}\right) \\
H_{32}\left(s_{1}, s_{2}, s_{3}\right)
\end{array}\right|=\left|\begin{array}{c}
-i_{N L 3} \\
i_{N L 3}
\end{array}\right|
$$

where $i_{N L 3}$ is the third order nonlinear current source for the two-dimensional conductance $I_{\text {stim }}$

$$
\begin{gathered}
i_{N L 3}=\frac{A_{g}}{3}\left(H_{11}\left(s_{1}\right) H_{22}\left(s_{2}, s_{3}\right)+H_{11}\left(s_{2}\right) H_{22}\left(s_{1}, s_{3}\right)+H_{11}\left(s_{3}\right) H_{22}\left(s_{1}, s_{2}\right)+\right. \\
\left.H_{12}\left(s_{1}\right) H_{21}\left(s_{2}, s_{3}\right)+H_{12}\left(s_{2}\right) H_{21}\left(s_{1}, s_{3}\right)+H_{12}\left(s_{3}\right) H_{21}\left(s_{1}, s_{2}\right)\right)
\end{gathered}
$$


By solving the equations, the third order Volterra kernels can be obtained as:

$$
\begin{aligned}
& H_{31}=\frac{-i_{N L 3}\left(G_{2}+s C_{2}\right)}{s^{2} C_{1} C_{2}++s\left(C_{2} G_{1}+C_{1} G_{2}+C_{2} G_{c}+C_{1} G_{p}\right)+\left(G_{1} G_{p}+G_{1} G_{2}+G_{2} G_{c}-G_{1} G_{p} \beta_{s p}\right)} \\
& H_{32}=\frac{i_{N L 32}\left(G_{1}-G_{1} \beta_{s p}+s C_{1}\right)}{s^{2} C_{1} C_{2}++s\left(C_{2} G_{1}+C_{1} G_{2}+C_{2} G_{c}+C_{1} G_{p}\right)+\left(G_{1} G_{p}+G_{1} G_{2}+G_{2} G_{c}-G_{1} G_{p} \beta_{s p}\right)}
\end{aligned}
$$

where

$$
s=s_{1}+s_{2}+s_{3}
$$

With the Volterra kernels $H_{12}, H_{22}$ and $H_{32}$ derived, harmonic distortions and intermodulation distortions can be obtained as:

$$
\begin{gathered}
\frac{H D_{2}}{C}=\frac{\frac{1}{2} A^{2} H_{22}(j \omega, j \omega)}{H_{12}(j \omega)} \\
\frac{I M_{2}}{C}=\frac{A_{1} A_{2} H_{22}\left(j \omega_{1}, \pm j \omega_{2}\right)}{H_{12}(j \omega)} \\
\frac{H D_{3}}{C}=\frac{\frac{1}{4} A^{3} H_{32}(j \omega, j \omega, j \omega)}{H_{12}(j \omega)} \\
\frac{I M_{3}}{C}=\frac{\frac{3}{4} A_{1}^{2} A_{2} H_{32}\left(j \omega_{1}, j \omega_{1}, \pm j \omega_{2}\right)}{H_{12}(j \omega)} .
\end{gathered}
$$

Having been discussed in Section 2.3.2, the transfer function $H_{11}$ and $H_{12}$ have complex poles which peaks the linear gain at the relaxation oscillation frequency $\omega_{0}$. Noticing that the denominators in equations (2.3.39) and (2.3.40) are similarly to the denominators in equations (2.3.35) and (2.3.36) and for the second order harmonic distortion,

$$
s=2 j \omega
$$

the transfer function $H_{11}$ and $H_{12}$ for the second order harmonic distortion have complex poles at $\omega_{0} / 2$. The $i_{N L 2}$ component has complex poles at $\omega_{0}$. It can be concluded that the second order harmonic distortion peaks at both the relaxation oscillation frequency $\omega_{0}$ and the half relaxation oscillation frequency $\omega_{0} / 2$.

Similarly, the denominators of the transfer function have the same form as before. It can be concluded that the third order harmonic distortion peaks at the relaxation oscillation 
frequency $\omega_{0}$ the half relaxation oscillation frequency $\omega_{0} / 2$ and one-third of the relaxation oscillation frequency $\omega_{0} / 3$.

In order to include the gain saturation effect, the gain compression in the modal gain expression of (2.3.31) is expanded as a Taylor series:

$$
S_{p}=(1+\delta \cdot P)^{-\frac{1}{2}}=M_{0}+M_{1} p+\ldots
$$

where

$$
M_{0}=\left(1+\delta^{n} P_{d c}^{n}\right)^{-\frac{1}{2}}
$$

and

$$
M_{1}=\frac{-\delta^{n}}{2\left(1+\delta^{n} P_{d c}^{n}\right)^{-1.5}} .
$$

The nonlinear component $I_{\text {stim }}$ is represented by:

$$
I_{s t i m}=A_{g}\left(C_{d c}^{n}+c-C_{t r}^{n}\right)\left(P_{d c}^{n}+p\right) S_{p} .
$$

After expanding (2.3.55) and keeping the first three low order terms, the circuit parameters $G_{c}, G_{p}$ and $A_{g}$ are updated as

$$
\begin{gathered}
G_{c}^{\prime}=G_{c} \cdot M_{0} \\
G_{p}^{\prime}=G_{p} \cdot\left(M_{0}+M_{1} P_{d c}\right) \\
A_{g}^{\prime}=A_{g} \cdot\left(M_{0}+M_{1} P_{d c}\right) .
\end{gathered}
$$

Applying these updated circuit parameters to Volterra kernel expressions in (2.3.35), (2.3.36), (2.3.39), (2.3.40), (2.3.44) and (2.3.45), the circuit transfer functions including gain saturation can be obtained. Figure 2.14, 2.15, 2.16 and 2.17 show the comparison of the linear, second and third harmonic components, and the third order intermodulation components between the ADS simulation results and the computed Volterra kernels with $30 \mathrm{~mA}$ bias current and $5 \mathrm{~mA} \mathrm{RF}$ input signal. From these figures, it can be concluded that the gain saturation reduces the gain and the distortion peaking due to the relaxation oscillation. 


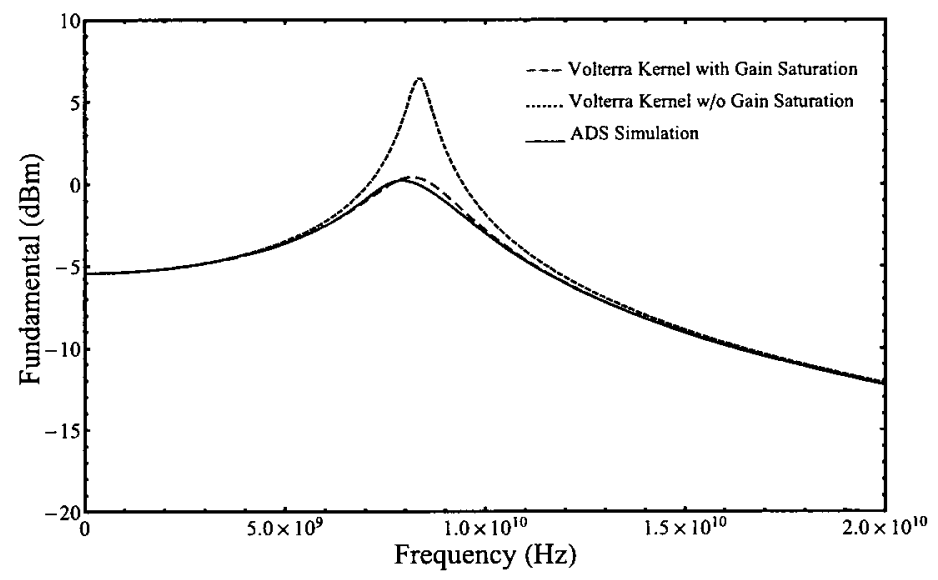

Figure 2.14: Linear component of laser output.

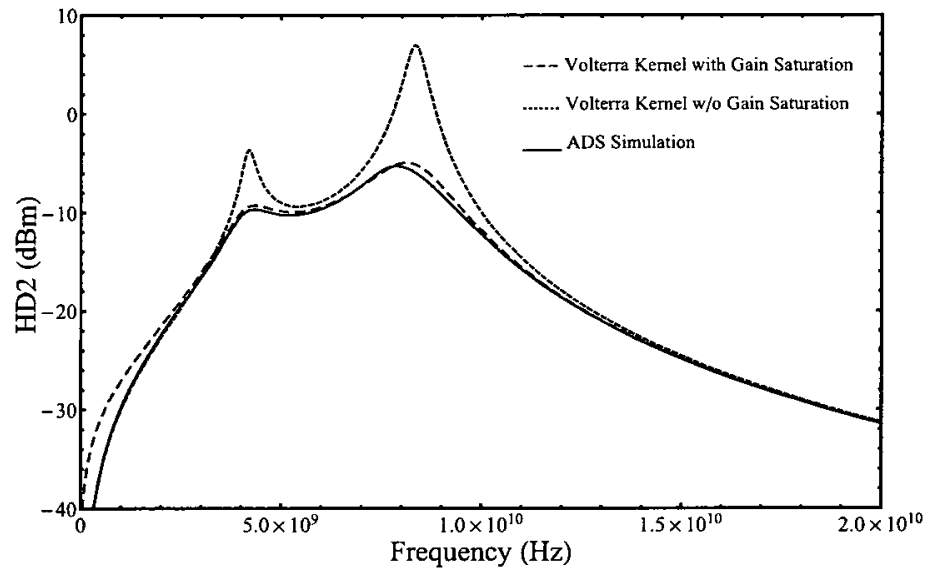

Figure 2.15: Second order harmonic distortion of laser output. 


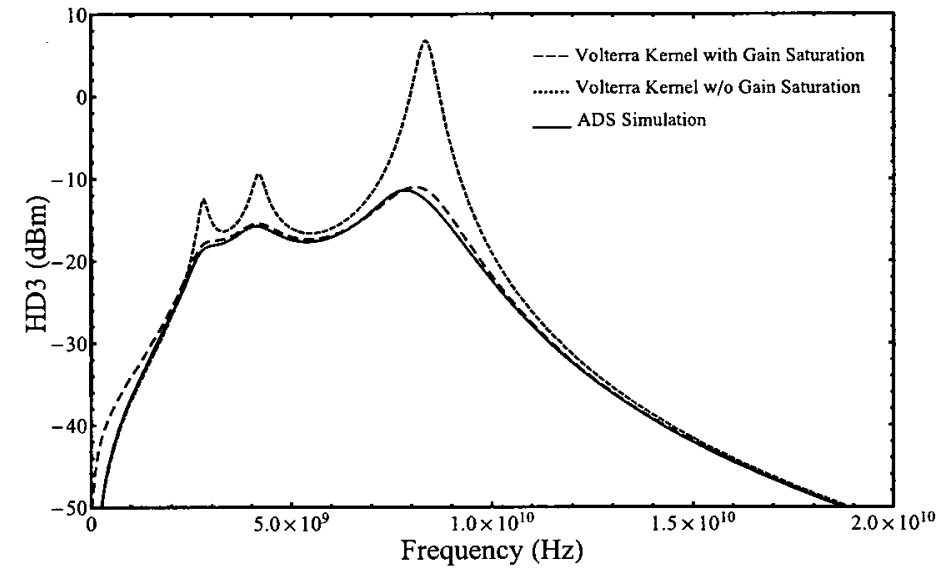

Figure 2.16: Third order harmonic distortion of laser output.

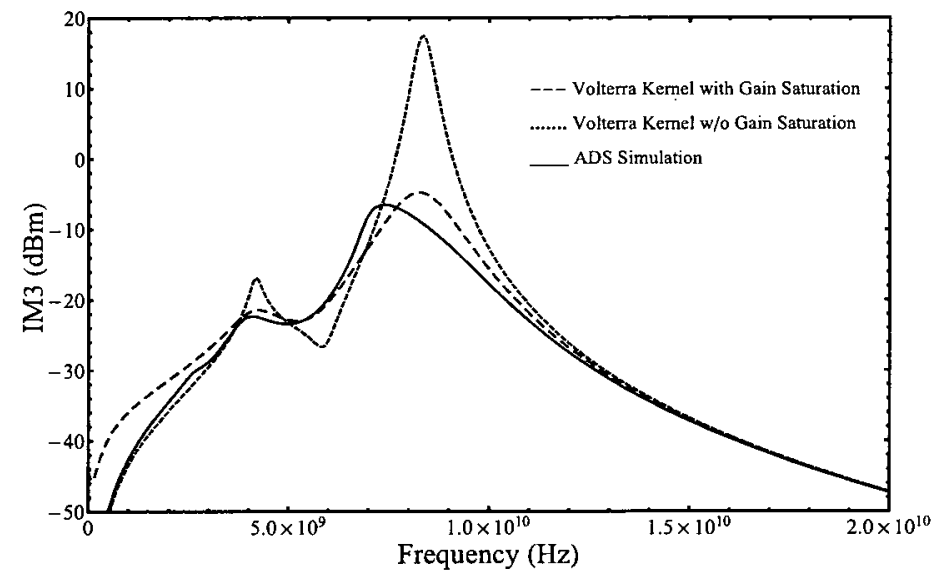

Figure 2.17: Third order intermodulation distortion of laser output. 


\subsection{Laser Inverse Model}

The desired predistortion function is the inverse of the laser transfer function. It can be derived from the laser Volterra kernels derived in the previous section. However, the resultant predistortion function would be too complex to be useful. Due to certain features of the rate equations (2.2.1) and (2.2.2), the laser inverse model can be derived analytically as described in [35]. The procedure will be briefly described in this section.

From equation (2.2.2), the carrier density can be obtained as

$$
C=\frac{\frac{d P}{d t}+\frac{P}{\tau_{p h}}+\frac{\Gamma v_{g} g C_{t r} P}{\sqrt{1+\delta P}}}{\frac{\Gamma \beta_{s p}}{\tau_{e}}+\frac{\Gamma v_{g} g P}{\sqrt{1+\delta P}}} .
$$

From equation (2.2.1), the input current can be obtained as

$$
I=\left(\frac{d C}{d t}+\frac{C}{\tau_{e}}+\frac{v_{g} g\left(C-C_{t r}\right) P}{\sqrt{1+\delta P}}\right) q V_{c} .
$$

Substituting (2.4.1) into (2.4.2), the laser inverse function

$$
I(t)=f(P(t))
$$

can be obtained. Applying Tyler series expansion to the expression, a polynomial form of $f(P)$ can be obtained. Neglecting higher order terms, the laser inverse function can be written as

$$
\begin{aligned}
I=I_{d c} & +m_{0}\left(P_{a c}\right)+m_{1}\left(P_{a c}\right)^{\prime}+m_{2}\left(P_{a c}\right)^{\prime \prime} \\
& +m_{3}\left(P_{a c}^{2}\right)+m_{4}\left(P_{a c}^{2}\right)^{\prime}+m_{5}\left(P_{a c}^{2}\right)^{\prime \prime} \\
& +m_{6}\left(P_{a c}^{3}\right)+m_{7}\left(P_{a c}^{3}\right)^{\prime}+m_{8}\left(P_{a c}^{3}\right)^{\prime \prime}
\end{aligned}
$$

where the coefficients $m_{0} \ldots m_{8}$ are functions of the laser parameters and the dc output $P_{d c}$. Detailed expression of $m_{0} \ldots m_{8}$ can be found in [35] and reproduced in Appendix A of this thesis. This laser inverse function can be represented by the block diagram shown in Figure 2.18. It contains three signal paths formed by linear, square and cubic functions followed by linear filters, where

$$
\begin{aligned}
& h_{1}(s)=m_{0}+m_{1} s+m_{2} s^{2} \\
& h_{2}(s)=m_{3}+m_{4} s+m_{5} s^{2} \\
& h_{3}(s)=m_{6}+m_{7} s+m_{8} s^{2} .
\end{aligned}
$$




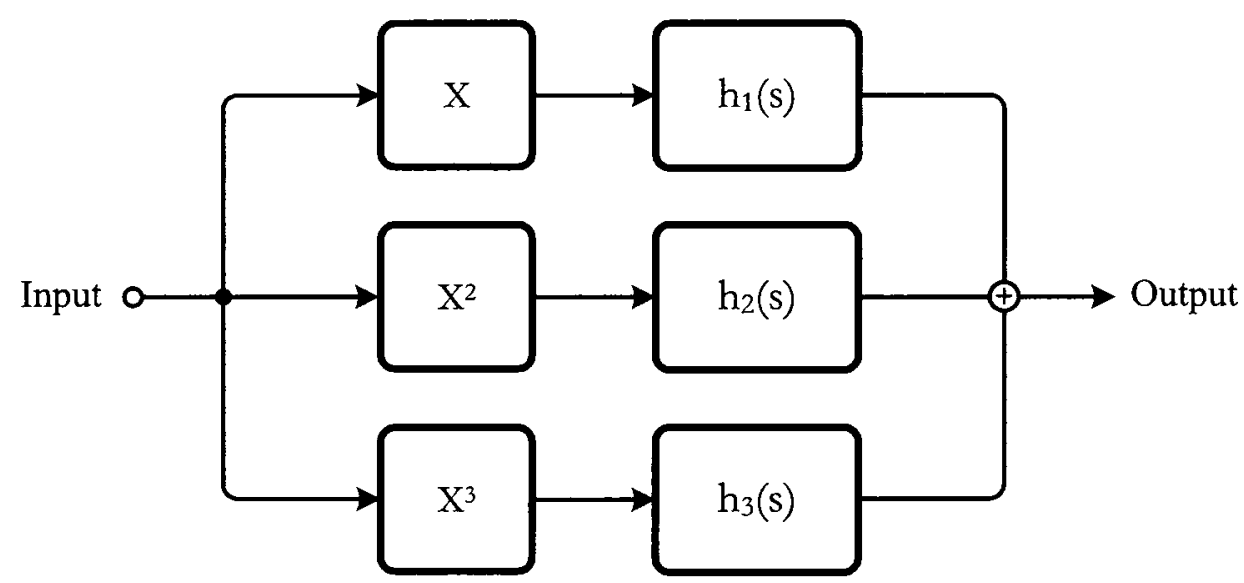

Figure 2.18: Block diagram of laser inverse model (predistorter model).

\subsection{Inverse Model Based Laser Model}

From the laser inverse function of (2.4.4) and (2.4.5), the Volterra kernels of the inverse model can be obtained as

$$
\begin{aligned}
& H_{1}(s)=h_{1}(s)=m_{0}+m_{1} s+m_{2} s^{2} \\
& H_{2}\left(s_{1}, s_{2}\right)=h_{2}\left(s_{1}+s_{2}\right)=m_{3}+m_{4}\left(s_{1}+s_{2}\right)+m_{5}\left(s_{1}+s_{2}\right)^{2} \\
& H_{3}\left(s_{1}, s_{2}, s_{3}\right)=h_{3}\left(s_{1}+s_{2}+s_{3}\right)=m_{6}+m_{7}\left(s_{1}+s_{2}+s_{3}\right)+m_{8}\left(s_{1}+s_{2}+s_{3}\right)^{2} .
\end{aligned}
$$

Arranging laser inverse model and laser model as a cascade of two third order stages as

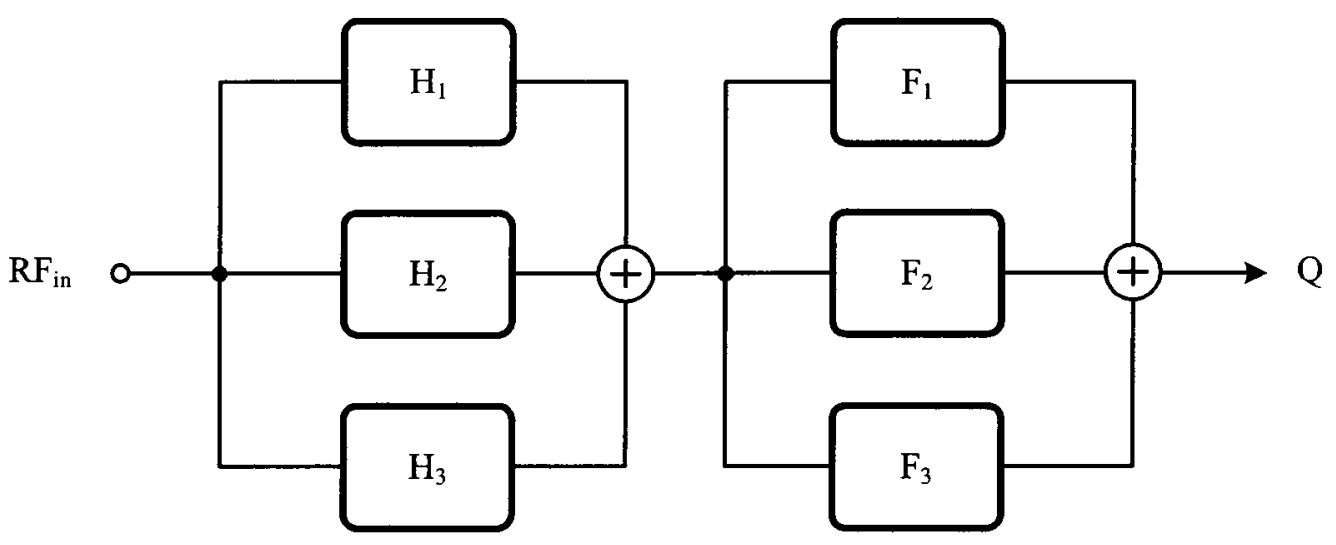

Figure 2.19: A cascade of two stages. 
shown in Figure 2.19, the output in Volterra kernels can be obtained as

$$
\begin{aligned}
& Q_{1}\left(s_{1}\right)=H_{1}\left(s_{1}\right) F_{1}\left(s_{1}\right) \\
& \begin{aligned}
Q_{2}\left(s_{1}, s_{2}\right)=H_{2} & \left(s_{1}, s_{2}\right) F_{1}\left(s_{1}+s_{2}\right)+H_{1}\left(s_{1}\right) H_{1}\left(s_{2}\right) F_{1}\left(s_{1}, s_{2}\right) \\
Q_{3}\left(s_{1}, s_{2}, s_{3}\right)= & H_{3}\left(s_{1}, s_{2}, s_{3}\right) F_{1}\left(s_{1}+s_{2}+s_{3}\right)+H_{1}\left(s_{1}\right) H_{1}\left(s_{2}\right) H_{1}\left(s_{3}\right) F_{3}\left(s_{1}, s_{2}, s_{3}\right) \\
& +\frac{2}{3} H_{1}\left(s_{1}\right) H_{2}\left(s_{2}, s_{3}\right) F_{2}\left(s_{1}, s_{2}+s_{3}\right) \\
& +\frac{2}{3} H_{1}\left(s_{2}\right) H_{2}\left(s_{1}, s_{3}\right) F_{2}\left(s_{2}, s_{1}+s_{3}\right) \\
& +\frac{2}{3} H_{1}\left(s_{3}\right) H_{2}\left(s_{1}, s_{2}\right) F_{2}\left(s_{3}, s_{1}+s_{2}\right)
\end{aligned}
\end{aligned}
$$

Being the outputs of a cascade of the original model and the inverse model,

$$
\begin{aligned}
& Q_{1}\left(s_{1}\right)=1 \\
& Q_{2}\left(s_{1}, s_{2}\right)=0 \\
& Q_{3}\left(s_{1}, s_{2}, s_{3}\right)=0
\end{aligned}
$$

should be satisfied. Therefore the Volterra kernel for the laser model can be obtained as

$$
\begin{aligned}
& F_{1}(s)=\frac{1}{H_{1}(s)} \\
& F_{2}\left(s_{1}, s_{2}\right)=\frac{-H_{2}\left(s_{1}, s_{2}\right)}{H_{1}\left(s_{1}\right) H_{1}\left(s_{2}\right) H_{1}\left(s_{1}+s_{2}\right)} \\
& F_{3}\left(s_{1}, s_{2}, s_{3}\right)=\frac{1}{H_{1}\left(s_{1}\right) H_{1}\left(s_{2}\right) H_{1}\left(s_{3}\right) H_{1}\left(s_{1}+s_{2}+s_{3}\right)} \\
& \cdot\left(\frac{2 H_{2}\left(s_{1}, s_{2}+s_{3}\right) H_{2}\left(s_{2}, s_{3}\right)}{3 H_{1}\left(s_{2}+s_{3}\right)}+\frac{2 H_{2}\left(s_{2}, s_{1}+s_{3}\right) H_{2}\left(s_{1}, s_{3}\right)}{3 H_{1}\left(s_{1}+s_{3}\right)}\right. \\
& \left.+\frac{2 H_{2}\left(s_{3}, s_{1}+s_{2}\right) H_{2}\left(s_{1}, s_{2}\right)}{3 H_{1}\left(s_{1}+s_{2}\right)}-H_{3}\left(s_{1}, s_{2}, s_{3}\right)\right) \text {. }
\end{aligned}
$$

These Volterra kernels are similar to the ones derived in section 2.3.3 using circuit analysis techniques, but contain third order gain saturation effects. The comparison between the inverse model based laser model and the ADS simulation results are shown in Figure 2.20, 2.21, 2.22 and 2.23. Compared with the results in Figure 2.14, 2.15, 2.16 and 2.17 obtained in the previous section, this laser model gives more accurate results as it considers higher order gain compression. 


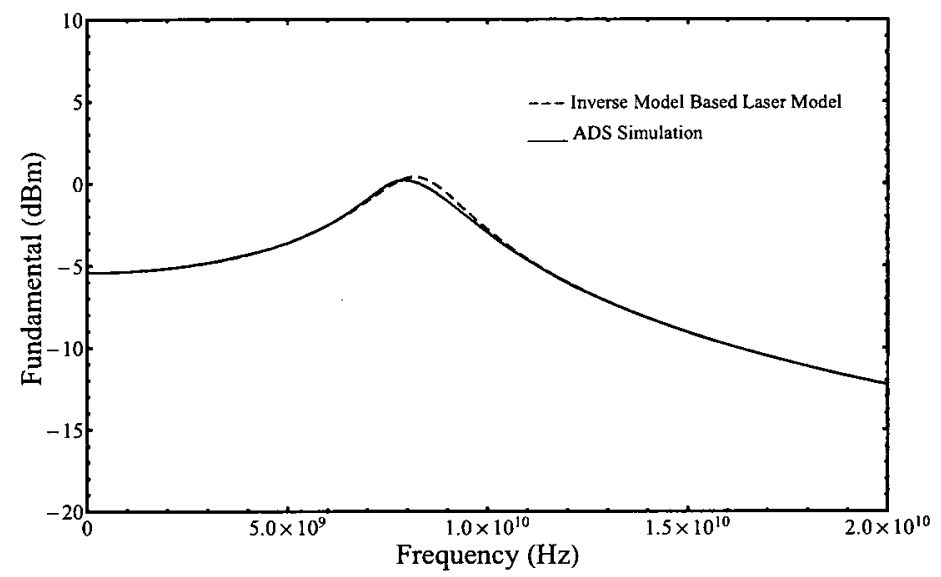

Figure 2.20: Linear component of laser output.

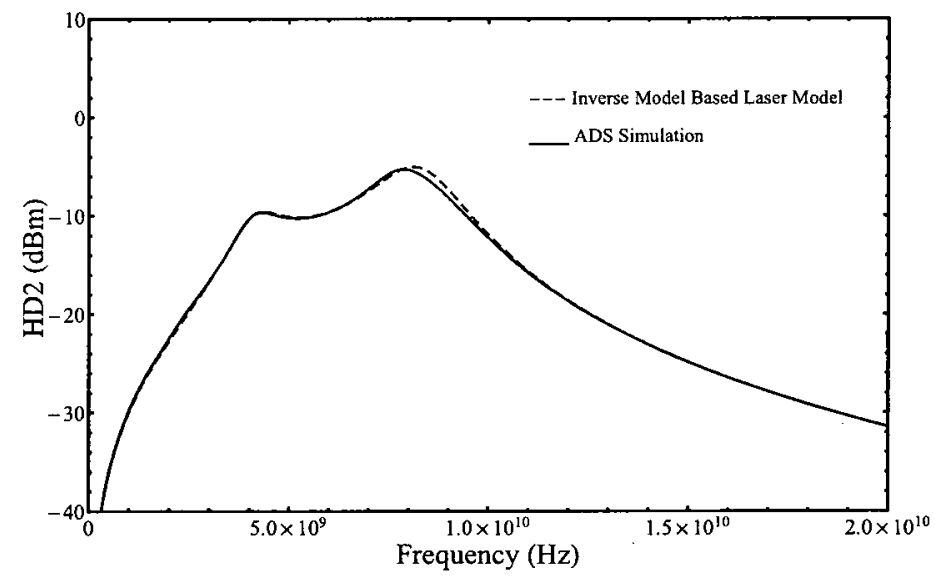

Figure 2.21: Second order harmonic distortion of laser output. 


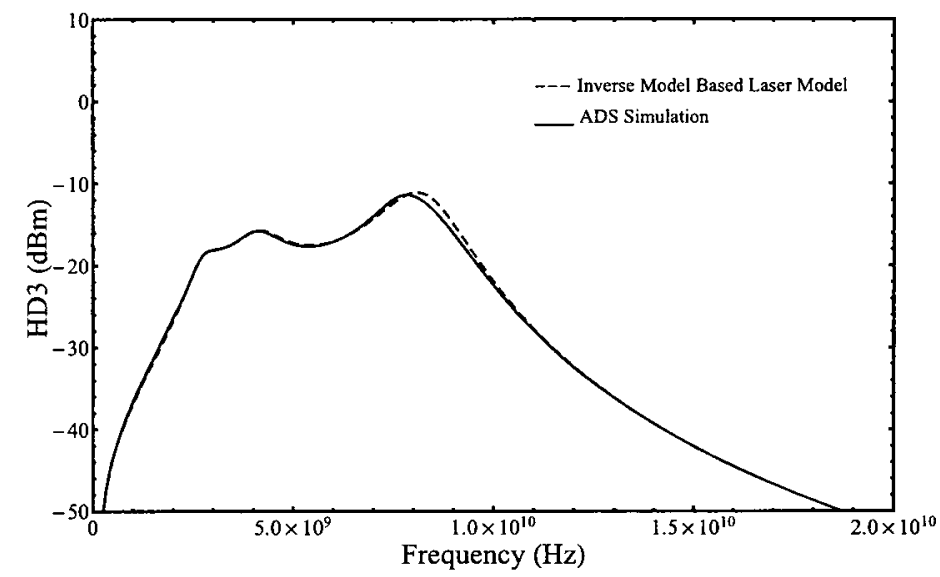

Figure 2.22: Third order harmonic distortion of laser output.

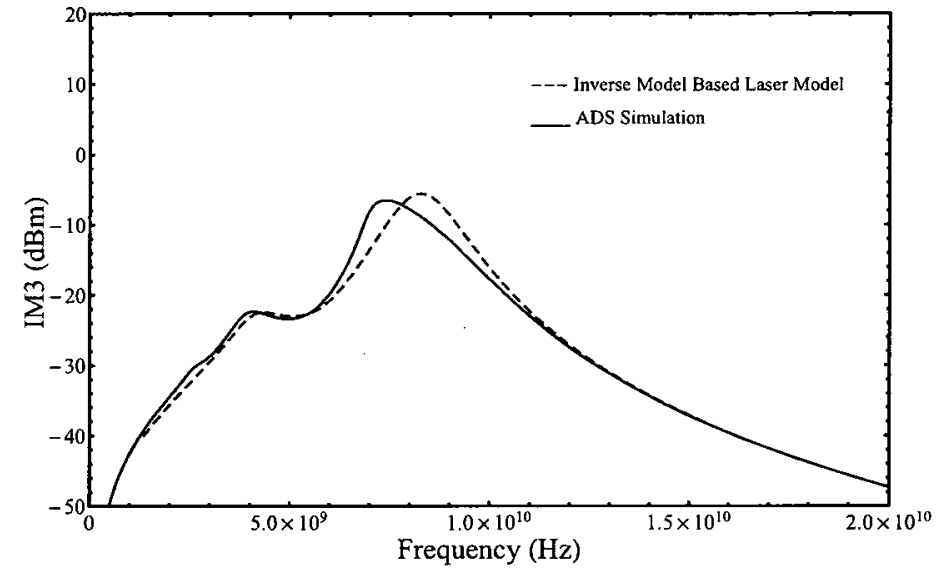

Figure 2.23: Third order intermodulation distortion of laser output. 


\subsection{Review of Laser Linearization Techniques}

Linearity is an important consideration in the design of analog light-wave transmission systems. For delivering a service with satisfactory quality it is mandatory to keep the distortion below certain level. For example, the CATV application requires a carrier to distortion ratio better than $50 \mathrm{~dB}$; GSM pico-cellular and micro-cellular requires a satisfactory dynamic range of $40-55 \mathrm{~dB}$ and $80-90 \mathrm{~dB}$ respectively $[3,4]$. A variety of linearization techniques for semiconductor lasers have been introduced and can be classified as: optoelectronic feedback and optical feed-forward [5-10,44,45], phase shift linearization [46], external light injection [47-49], post-distortion [11-13,50,51] and pre-distortion [14-19,25,35,52,53].

\subsubsection{Optoelectronic Feedback}

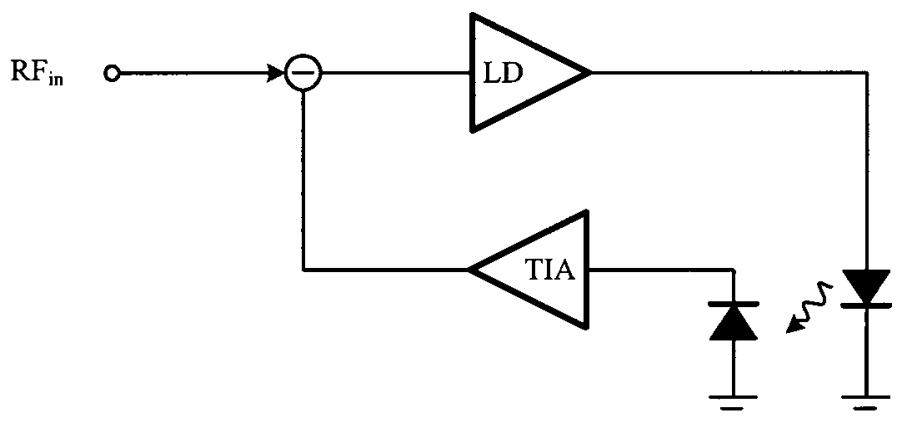

Figure 2.24: Optoelectronic feedback linearization.

The optoelectronic feedback is based on the conventional negative feedback mechanism. The output with distortion is fed back negatively to the input by a linear path. If the open-loop gain is large enough, the overall linearity will be determined by the feedback path, hence improving the linearity. Figure 2.24 shows a simplified block diagram of a optoelectronic feedback system. It consists of a monitoring photodiode and a transimpedance amplifier. The optical output is detected by the photodiode and amplified by the transimpedance amplifier. The photodiode nonlinearities can usually be neglected for low laser diode power, such as in cellular fiber optic applications. Several experiments have been carried out during the 1970s and 1980s [44,45]. It has been demonstrated a reduction of 10 
$\mathrm{dB}$ in the 2 nd and $3 \mathrm{rd}$ order harmonic distortions. However, this technique is not suited for high frequency and wideband applications. For such applications, tight requirements on gain bandwidth and time delay of the loop must be met. The linearity enhancement also limited by the feedback path.

\subsubsection{Optical Feed-forward}

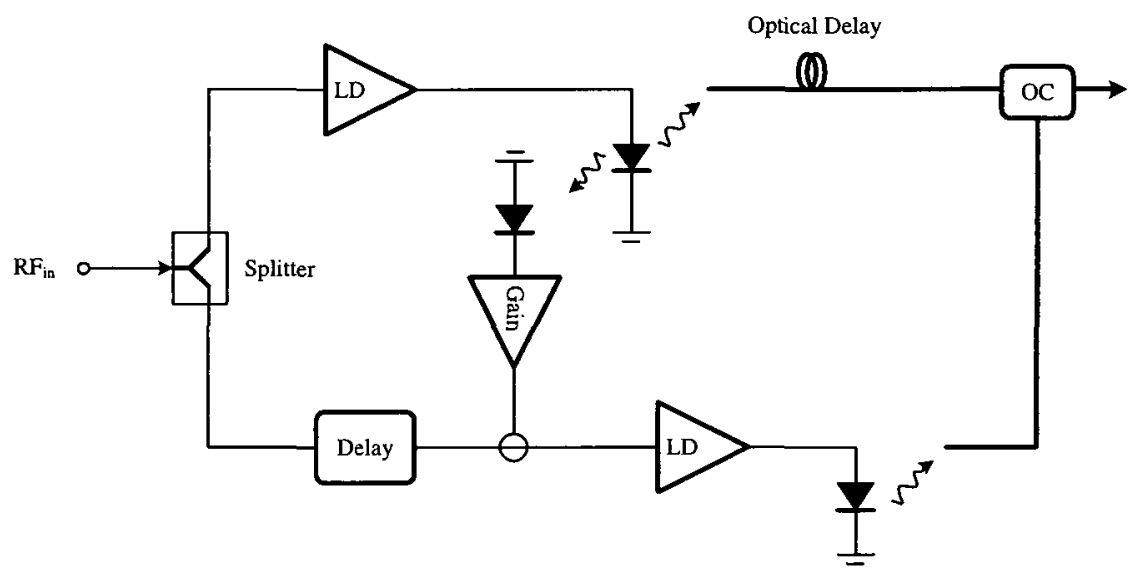

Figure 2.25: Optical feed-forward linearization.

Figure 2.25 shows the block diagram of a optical feed-forward system. The input signal is first split into two paths. The first path directly drives the laser diode. The second path is used as a reference of ideal output. The optical output from the first path is detected by a photodiode and then compared with delayed signal in the second path. The error signal drives another laser diode to produce the optical compensation injected directly into the fiber. Ideally, this technique cancels the nonlinearity of the laser diode and the combined output is the linear representation of the input. This technique has the advantage of reducing laser noise as well as distortion. Typically 10-20 $\mathrm{dB}$ reduction of the 2nd and third order distortion can be obtained using optical feed-forward technique [5-10]. However, accurate time-delay arrangement between the two signal paths is critical for the distortion cancelation and the second laser diode should be as linear as possible. Cost and matching problems are the main drawbacks of this technique. 


\subsubsection{Phase Shift Linearization}

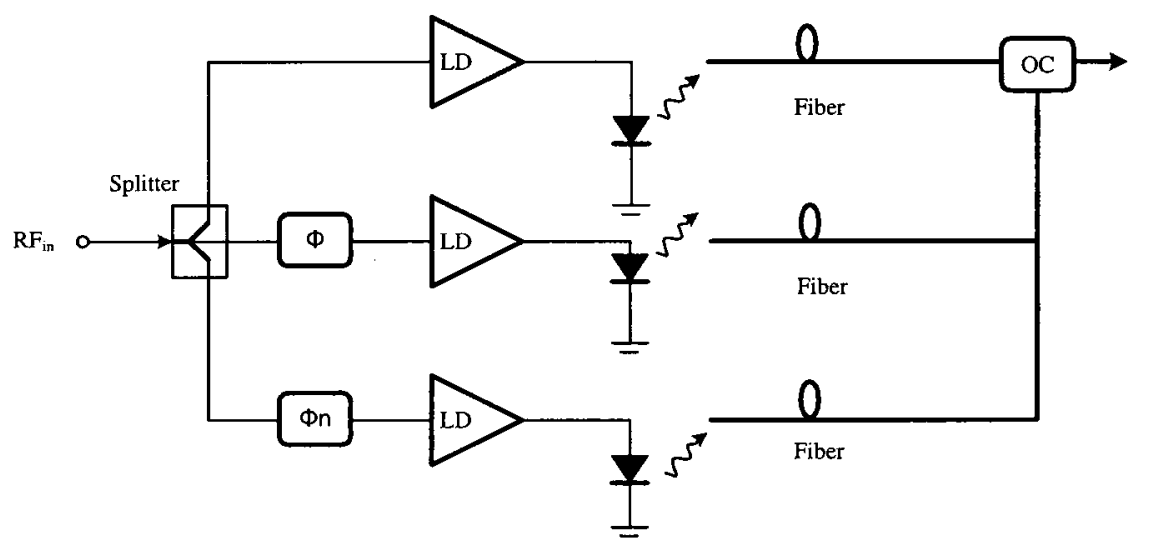

Figure 2.26: Phase shift modulation linearization.

The phase shift linearization technique was introduced by J. Strauss in 1977 [46]. Figure 2.26 shows the block diagram of a phase shift linearization system. A phase shift passing through a nonlinear system will be multiplied by the distortion order. By properly arranging the phase shift between the different paths of the input signal, specific order of distortion can be canceled at the combined optical output. For example, $90^{\circ}$ phase difference between two paths can remove the 2 nd order distortion and a $n$-th order distortion can be canceled by a phase difference of $180^{\circ} / n$. Strauss reported a $25 \mathrm{~dB}$ improvement in the second order distortion. This technique is generally not cost-effective as an additional laser diode is required for each cancelation order and the linear component also reduced due to the phase shift. Also, good matching of the laser diodes is required for high performance.

\subsubsection{External Light Injection}

The theoretical analysis of laser rate equations have suggested that, by moving the relaxation oscillation to a much higher frequency, the nonlinear distortion can be greatly reduced. Optical injection locking has been demonstrated as a very effective way of increasing the relaxation oscillation frequency. Therefore, applying external coherent light injection to the laser cavity will potentially increase the laser linearity. G. Yabre introduced 


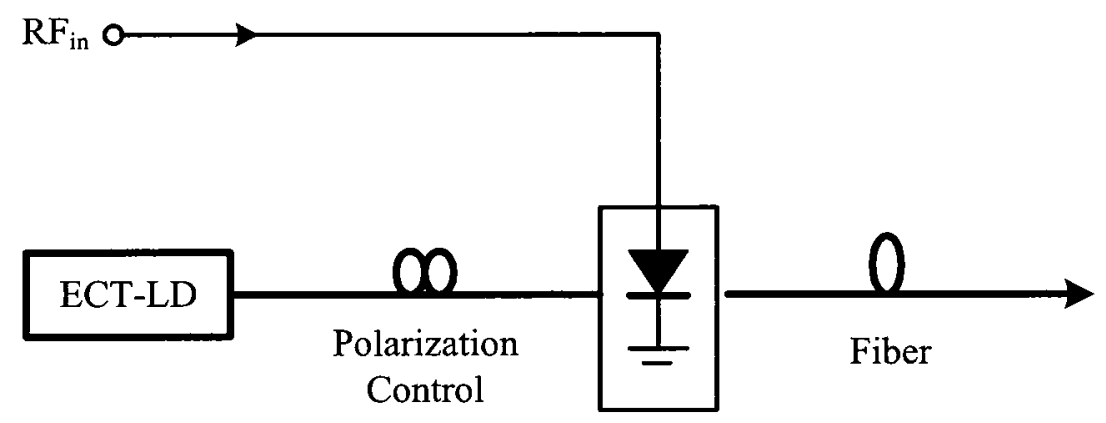

Figure 2.27: External light injection.

the theoretical investigation of the 2nd and third distortions in an injection-locked laser in 1977 [49]. Figure 2.27 shows the simplified block diagram of the linearization technique using external light injection, where an external cavity tunable laser diode (ECT-LD) is used in conjunction with a direct-modulated lase diode. The first experimental demonstration of linearity improvement using optical injection locking was reported in $[47,48]$. Their results shows that more than $10 \mathrm{~dB}$ reduction of 2 nd harmonic distortion nonlinearity can be obtained for a modulation frequency from 1 to $3 \mathrm{GHz}$.

\subsubsection{Postdistortion}

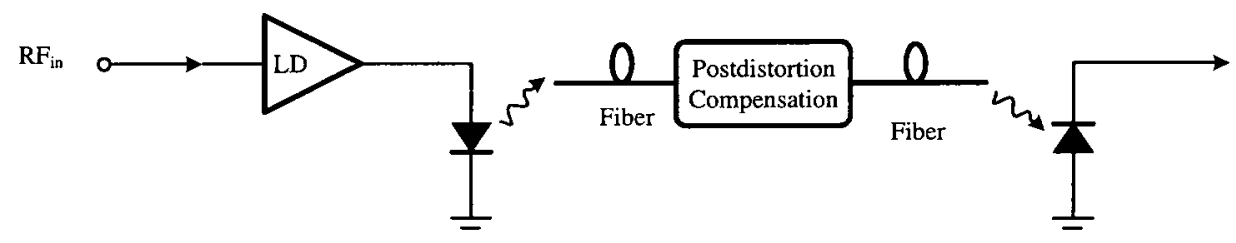

Figure 2.28: Optical postdistortion.

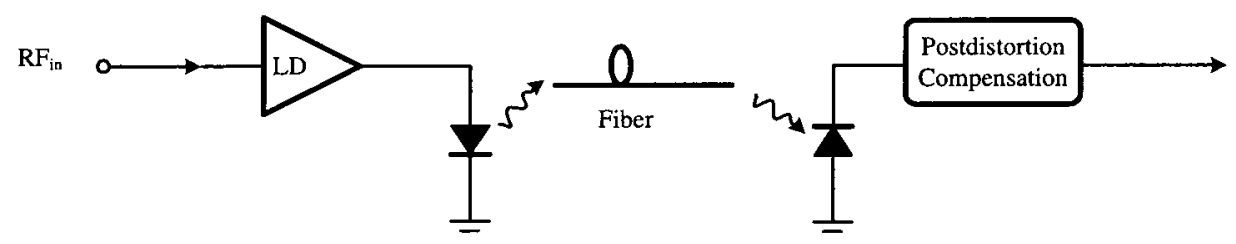

Figure 2.29: Electrical postdistortion. 
Postdistortion is a direct compensation technique which is similar to the feedforward technique which has no feedback control path. Postdistortion compensation functions as the inverse of the laser current/light characteristic placed after the laser diode - either using optical compensation or electronic compensation depending on the location of the postdistortion compensation. Figure 2.28 and 2.29 show the simplified block diagram of the two postdistortion compensation scheme. Some success has been demonstrated with optical postdistortion in [50] and with electrical postdistortion in [51]. However, post-distortion for wideband applications is rare, except for some theoretical studies in the literature [11-13].

\subsubsection{Predistortion}

Predistortion is another direct compensation technique. Instead of being placed after the laser diode as in the postdistortion linearization scheme, the predistortion compensation block is inserted somewhere in front of the laser diode. Predistortion blocks can be designed based on Volterra models to invert the light-current characteristic curve of a laser diode, thus compensating its nonlinearities. The basic structure of a 4 th order predistortion implementation is shown in Figure 2.30. The input signal splits into several paths. The linear path provides the dominate signal to drive the laser diode. The other paths provides correction signals which are equal in amplitude, but opposite in phase, to the undesired distortion introduced by the laser nonlinearity. $X^{2}, X^{3}$ and $X^{4}$ provide the required distortion. $\Phi_{1}, \Phi_{2}, \Phi_{3}$ are used to adjust the phase to the desired opposite value and $G_{1}$, $G_{2}$ and $G_{3}$ are used to adjust the amplitude.

Compared with other linearization techniques, predistortion technique offers a few advantages: 1) the predistortion compensation is implemented on the electrical domain, hence eliminating the use of expensive optical components; 2) it offers wideband compensation with simpler configuration; 3 ) it is an attractive option if designed using standard CMOS technology to reduce the cost and the size; 4) it has the potential to achieve the best distortion compensation according to the theoretical studies in [35]. A number of predistortion

circuits have been demonstrated. K. Asatani developed a predistorter for a LED [52]. 


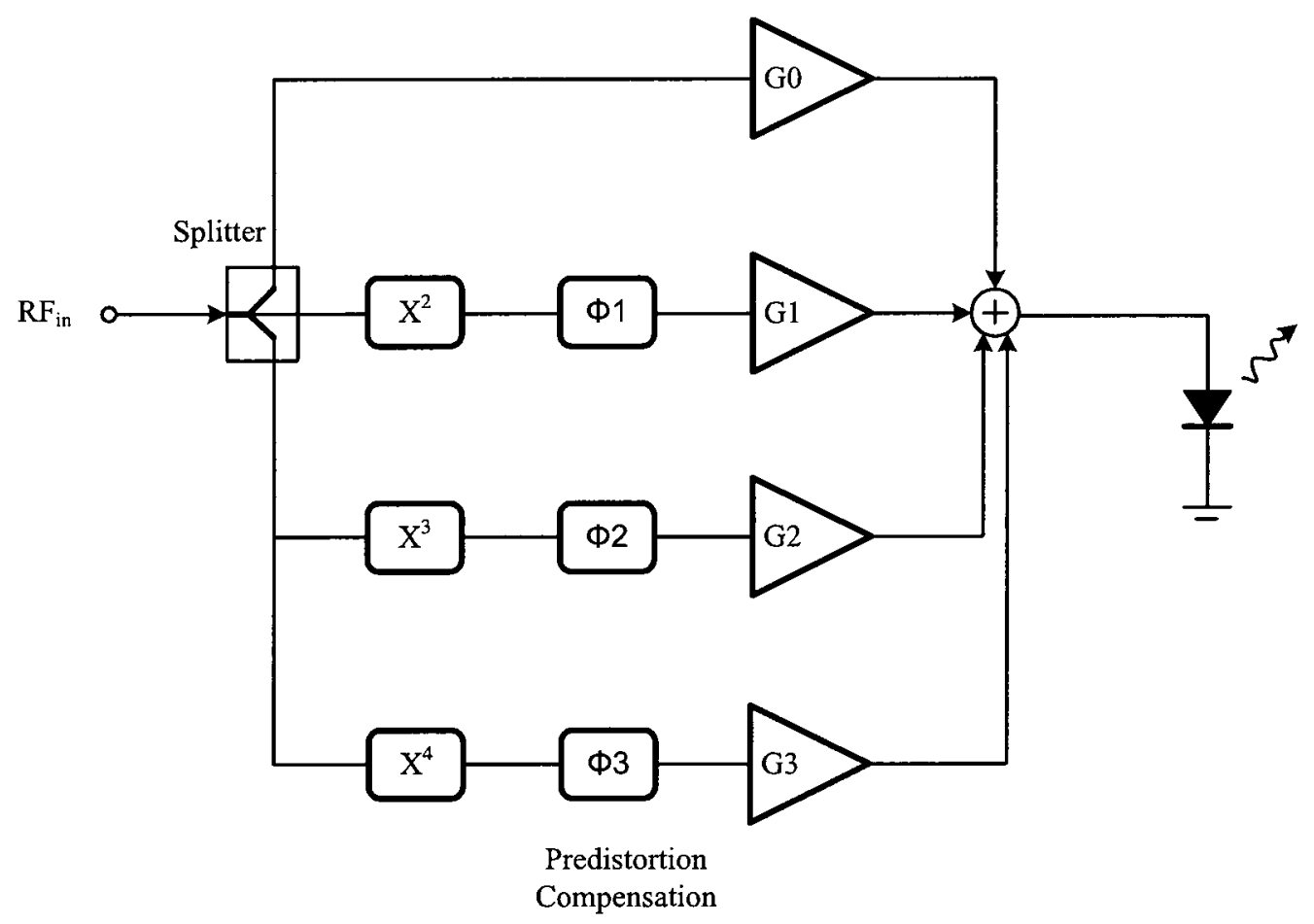

Figure 2.30: Predistortion. 
Their results showed an improvement of $19 \mathrm{~dB}$ and $21 \mathrm{~dB}$ for second and third order harmonic distortion respectively. L. Roselli developed a predistortion circuit using discrete components for multi-service telecommunication application [25]. Their results showed a $15 \mathrm{~dB}$ reduction in second order harmonic distortion and a $9 \mathrm{~dB}$ reduction in the third order harmonic distortion over the range of $500 \mathrm{MHz}-2 \mathrm{GHz}$. The theoretical work in [35] shows the potential of more than $50 \mathrm{~dB}$ reduction of the 2 nd and $3 \mathrm{rd}$ harmonic distortion using adaptive pre-distortion.

\subsection{Predistortion Challenges}

Electronic predistortion offers a cost-efficient solution of improving the linearity of the direct modulated laser. The objective of this research work is to develop a fully integrated predistortion circuit, using standard CMOS technology for radio-over-fiber application in $\mathrm{GHz}$ range. The small size and compact form factor will greatly reduce the fabrication cost and the full integration will greatly enhance its reliability for industrial applications. With the digital capability of CMOS technology, it will be possible to make a complete one-chip solution of a radio-over-fiber system.

However, there are design challenges in designing a CMOS laser predistortion circuit. First, the predistortion circuit by nature is nonlinear, it extends the signal bandwidth and generates several frequency bands. For example, in order to cancel the second order harmonic distortion and intermodulation distortion, the predistortion second order path should generate an output signal with double bandwidth at the second order harmonic band. In order to cancel the third order harmonic distortion and intermodulation distortion, the predistortion third order path should generate an output signal at both the fundamental frequency band and the third order harmonic band.

If considering a simple case that a single sinusoidal tone $f_{0}$ is used as the input, $d c, f_{0}, 2 f_{0}$ and $3 f_{0}$ frequency components will be obtained at the pre-distorter output and presented at the input of the laser diode. Through the linear term of the laser diode transfer function, $2 f_{0}$, and $3 f_{0}$ frequency components are mapped to the output at the same frequency to balance out the harmonic distortions, which are important since they would cause the 
inter-band interferences. $f_{0}$ frequency components from the odd order paths are used to balance out the intermodulation distortion. Through the second order nonlinearity of the laser diode, $2 f_{0}$ frequency component by $f_{0}$ frequency component will be down-mixed to the frequency $f_{0}$ and through the third order nonlinearity. Those $f_{0}$ tones are used to balance out the in-band distortion of the laser diode, which are critical for communication systems since they produce significant inter-channel interferences. For a third order compensation, the pre-distorter needs to work at up to 3 times the carrier frequency. The correction distortions generated by the distortion generators (for example, $X^{2} X^{3}$ and $X^{4}$ block in Figure 2.30) are usually small, which requires a large gain in order to generate the canceling signal with the right amplitude. The widely-known gain-bandwidth trade-off will greatly challenge the design of a predistortion circuit.

Secondly, the predistortion compensation relies on the perfect match between the predistorter and the laser to obtain good performance. It requires the matching of amplitudes and phases over a certain frequency range, and the alignment of the correction paths for an equal time delay. Those requirements impose a great difficulty on the circuit design. The semiconductor laser exhibits fluctuations in characteristics from component to component and changes in performance due to temperature fluctuation and aging. This necessitates adaptive calibration of the predistortion circuit in order to counteract the variation of the laser diode and correct the drift errors that would occur over time and temperature deviation.

\subsection{Summary}

In this chapter, the background knowledge of semiconductor laser was presented. The laser model and its implementation in the simulation environment has been described. The behavior of laser diode has been analyzed using Volterra series. The predistorter architecture is derived from laser inverse model. laser linearization techniques has been reviewed and design challenges for predistortion technique has been discussed. 


\section{Chapter 3}

\section{Predistortion Function Using Multiple Tank Approach}

The goal of this research is to develop a fully integrated laser pre-distortion circuit using standard CMOS technology and working at the $\mathrm{GHz}$ range. The first challenge in designing such a pre-distorter is to extend the circuit working bandwidth. Previous research on CMOS cubing circuit [54] has demonstrated the possibility as well as the difficulty of getting the circuit to work at the $\mathrm{GHz}$ ranges with a decent gain. This difficulty is due to the relatively low current driven capability and high parasitic capacitance of CMOS technology. It makes the situation even worse that the pre-distortion circuit needs to work at 2 or 3 times the fundamental frequency because of the nonlinear nature of this circuit. Secondly, the output frequency profile should be tunable in order to match the required shape which varies from component to component and depends on temperature.

\subsection{Predistorter Model}

Considering the laser inverse function of (2.4.4) and choosing zero $\mathrm{dB}$ gain, the predistorter function can be written as

$$
\begin{aligned}
i_{\text {out }}=I_{\text {out }}-I_{\text {bias }} & =\left(R F_{\text {in }}\right)+\frac{m_{1}}{m_{0}}\left(R F_{i n}\right)^{\prime}+\frac{m_{2}}{m_{0}}\left(R F_{i n}\right)^{\prime \prime} \\
& +\frac{m_{3}}{m_{0}^{2}}\left(R F_{i n}^{2}\right)+\frac{m_{4}}{m_{0}^{2}}\left(R F_{i n}^{2}\right)^{\prime}+\frac{m_{5}}{m_{0}^{2}}\left(R F_{i n}^{2}\right)^{\prime \prime} \\
& +\frac{m_{6}}{m_{0}^{3}}\left(R F_{i n}^{3}\right)+\frac{m_{7}}{m_{0}^{3}}\left(R F_{i n}^{3}\right)^{\prime}+\frac{m_{8}}{m_{0}^{3}}\left(R F_{i n}^{3}\right)^{\prime \prime}
\end{aligned}
$$


This predistortion function can be represented by the the block diagram shown in Figure 3.1 .

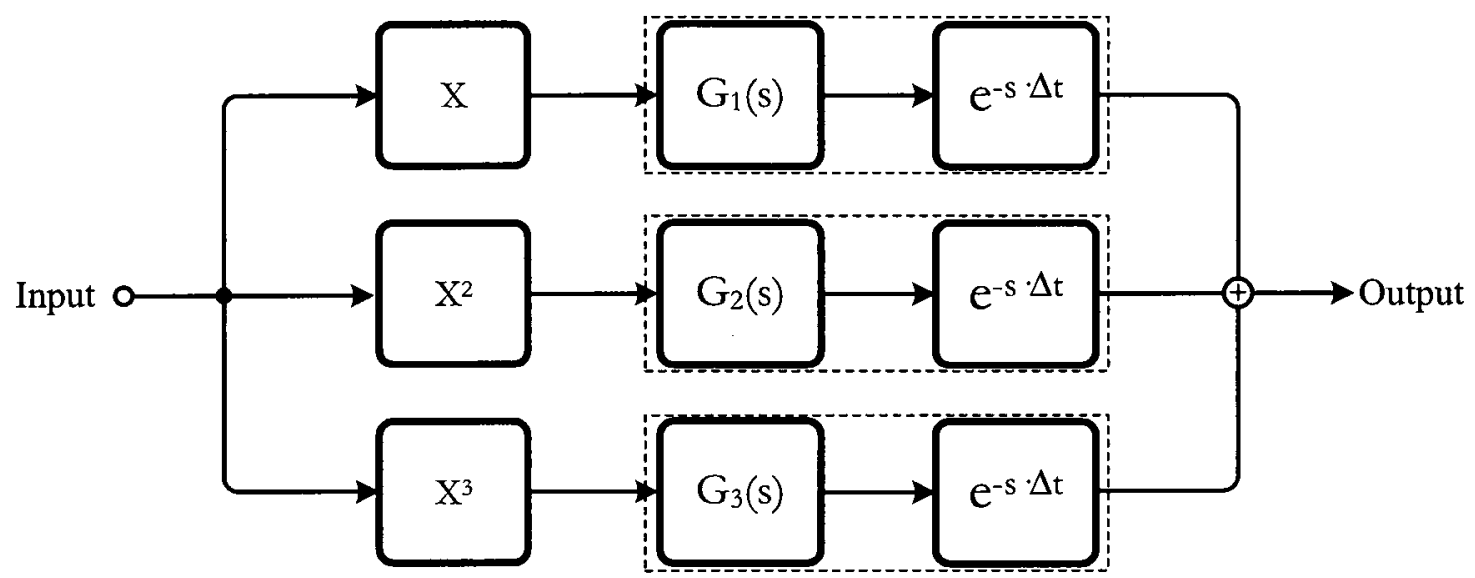

Figure 3.1: Block diagram of the laser predistorter.

It contains three signal paths: $X, X^{2}$ and $X^{3} . G_{1}(s), G_{2}(s)$ and $G_{3}(s)$ are the linear filters which shape the signal frequency profile of each path.

$$
\begin{aligned}
& G_{1}(s)=1+\frac{m_{1}}{m_{0}} s+\frac{m_{2}}{m_{0}} s^{2} \\
& G_{2}(s)=\frac{m_{3}}{m_{0}^{2}}+\frac{m_{4}}{m_{0}^{2}} s+\frac{m_{5}}{m_{0}^{2}} s^{2} \\
& G_{3}(s)=\frac{m_{6}}{m_{0}^{3}}+\frac{m_{7}}{m_{0}^{3}} s+\frac{m_{8}}{m_{0}^{3}} s^{2}
\end{aligned}
$$

$e^{-s \Delta t}$ is the time delay introduced for the reason of causality. To have a concise expression, three signal paths are combined in a vector format:

$$
\begin{aligned}
\vec{L}(s) & =\left(G_{1}(s) \vec{I}+G_{2}(s) \vec{J}+G_{3}(s) \vec{K}\right) e^{-s \Delta t} \\
& =G_{1}(s) e^{-s \Delta t} \vec{I}+G_{2}(s) e^{-s \Delta t} \vec{J}+G_{3}(s) e^{-s \Delta t} \vec{K}
\end{aligned}
$$

where $\Delta t$ is the time delay and vectors $\vec{I}, \vec{J}$ and $\vec{K}$ represent the $X, X^{2}$ and $X^{3}$ path respectively.

The $X^{2}$ and $X^{3}$ blocks are nonlinear components which extend the input signal's spectrum. Considering input signal $X(\omega)$ with a bandwidth of $\Delta \omega=\omega_{2}-\omega_{1}\left(\omega_{2}>\omega_{1}\right)$,

$$
X(\omega)=0 \quad \text { for }|\omega| \geqslant \omega_{2} \text { or }|\omega| \leqslant \omega_{1}
$$


the output spectrum of $X^{2}$ can be obtained by convolution integration

$$
\int_{-\infty}^{\infty} X(\tau) X(\omega-\tau) d \tau
$$

In order for the integration to be nonzero,

$$
\begin{array}{rlrl}
\omega_{2} & >|\tau|>\omega_{1} \\
\text { and } & \omega_{2} & >|\omega-\tau|>\omega_{1}
\end{array}
$$

should be satisfied. Therefore,

$$
\begin{aligned}
& 2 \omega_{2}>|\omega|>2 \omega_{1} \\
& \text { or }|\omega|<\omega_{2}-\omega_{1} .
\end{aligned}
$$

Similarly, in order for the output spectrum of $X^{3}$ to be nonzero,

$$
\begin{aligned}
& 3 \omega_{2}>|\omega|>3 \omega_{1} \\
\text { or } & 2 \omega_{2}-\omega_{1}>|\omega|>2 \omega_{1}-\omega_{2} .
\end{aligned}
$$

From (3.1.7) and (3.1.8), the output frequency range can be obtained and illustrated in Figure 3.2. It can be seen that $X^{2}$ extends the input spectrum to two bands with one around $\mathrm{DC}$ and the other around the second harmonic frequency and each has a bandwidth of $2 \Delta \omega$. $X^{3}$ extends the spectrum to two bands with one around the fundamental frequency and the other around the third harmonic frequency and each has a bandwidth of $3 \Delta \omega$.

Figure 3.3 shows the basic principle of predistortion: when an expansive block is cascaded with a compressive block, the overall linearity is improved. For semiconductor laser, the nonlinearity varies with frequency, therefore the predistortion transfer function should be adjusted for different frequencies as illustrated in Figure 3.4. While, in Figure 2.18, $X$, $X^{2}$ and $X^{3}$ block provide linear and distortion terms, the frequency profile shaping filter $G_{1}(s), G_{2}(s)$ and $G_{3}(s)$ adjust each term to achieve the required compression or expansion for different frequencies. More details of the predistortion function can be explained by Volterra transfer functions. Viewing the predistorter with the laser as a cascade of two 


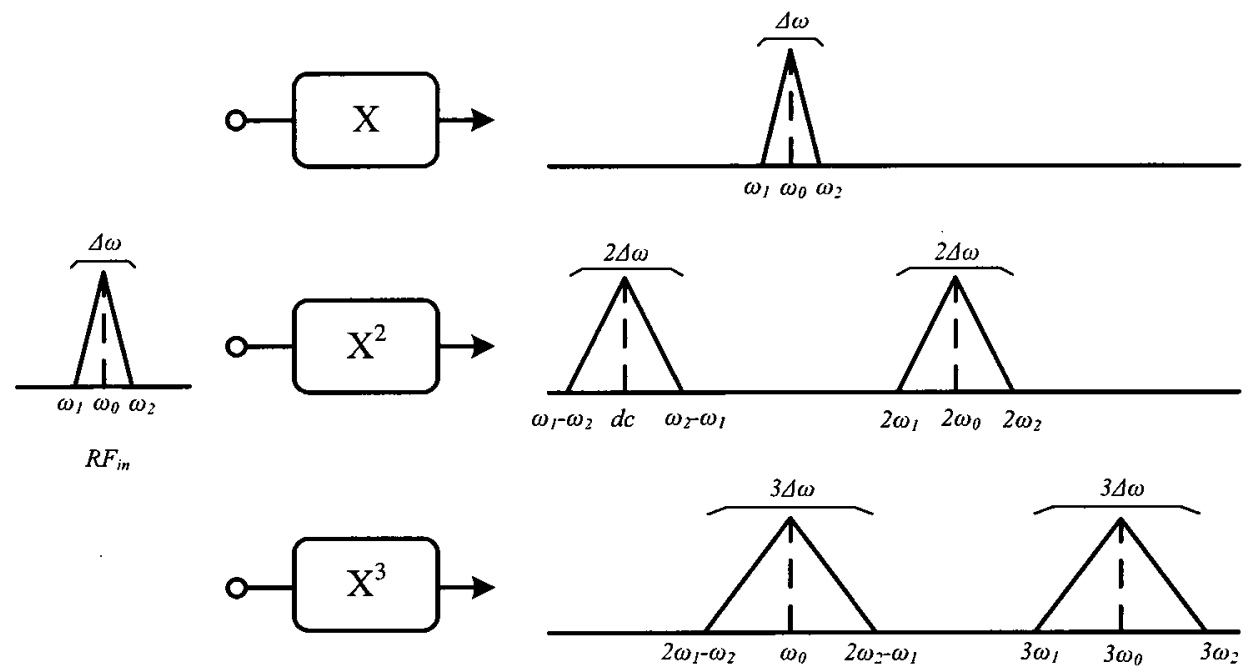

Figure 3.2: Output spectrum of $X X^{2}$ and $X^{3}$ components.

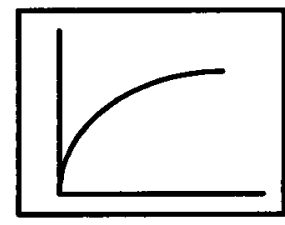

Predistortion

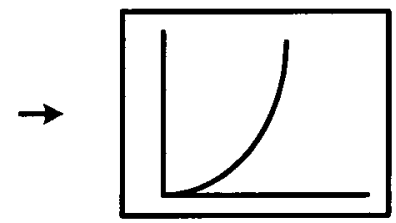

Laser Diode

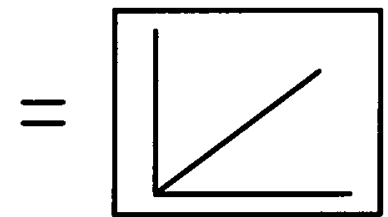

Overall

Figure 3.3: Predistortion principle.

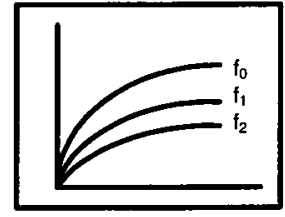

Predistortion

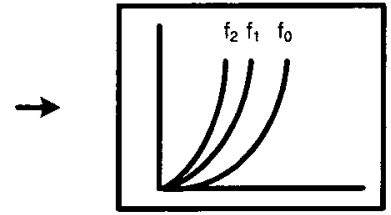

Laser Diode

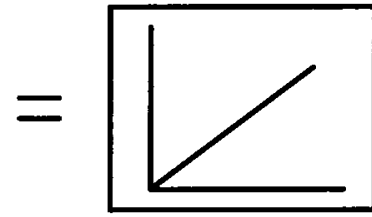

Overall

Figure 3.4: Laser predistortion principle. 


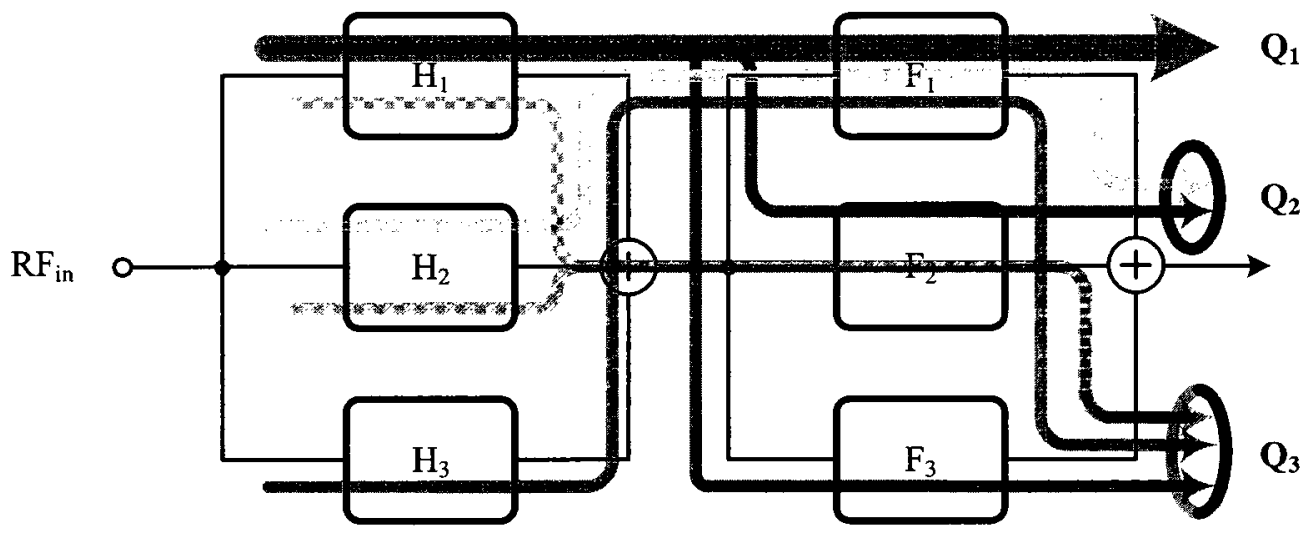

Figure 3.5: Distortion cancelation in a cascade of two stages.

stages shown in Figure 3.5, the Volterra kernels up to third order for the whole system are

$$
\begin{aligned}
& Q_{1}\left(s_{1}\right)=H_{1}\left(s_{1}\right) F_{1}\left(s_{1}\right) \\
& \begin{aligned}
Q_{2}\left(s_{1}, s_{2}\right)=H_{2} & \left(s_{1}, s_{2}\right) F_{1}\left(s_{1}+s_{2}\right)+H_{1}\left(s_{1}\right) H_{1}\left(s_{2}\right) F_{1}\left(s_{1}, s_{2}\right) \\
Q_{3}\left(s_{1}, s_{2}, s_{3}\right)= & H_{3}\left(s_{1}, s_{2}, s_{3}\right) F_{1}\left(s_{1}+s_{2}+s_{3}\right)+H_{1}\left(s_{1}\right) H_{1}\left(s_{2}\right) H_{1}\left(s_{3}\right) F_{3}\left(s_{1}, s_{2}, s_{3}\right) \\
& +\frac{2}{3} H_{1}\left(s_{1}\right) H_{2}\left(s_{2}, s_{3}\right) F_{2}\left(s_{1}, s_{2}+s_{3}\right) \\
& +\frac{2}{3} H_{1}\left(s_{2}\right) H_{2}\left(s_{1}, s_{3}\right) F_{2}\left(s_{2}, s_{1}+s_{3}\right) \\
& +\frac{2}{3} H_{1}\left(s_{3}\right) H_{2}\left(s_{1}, s_{2}\right) F_{2}\left(s_{3}, s_{1}+s_{2}\right)
\end{aligned}
\end{aligned}
$$

It is graphically illustrated in Figure 3.5 that 1) the second order distortion of the laser are canceled by the second order distortion of the predistorter through the linear term of the laser; 2) the last three terms in (3.1.9) is the third order laser distortion induced by the second order predistorter distortion; the third order predistorter distortion is used to cancel both the laser distortion and the induced distortion.

\subsection{Proposed Multiple Tank Technique}

Designing distortion generators $\left(X^{2}\right.$ and $\left.X^{3}\right)$ with a large operating bandwidth and an acceptable gain is usually difficult. The distortion levels of the distortion generators are usually low as they are produced by devices, mostly, in a process technology which are optimized for linear operations. This necessitates the subsequent frequency profile shaping 
filters with sufficient gain in order to achieve the desired distortion amplitude. As discussed in previous section, the spectrum of the predistorter output has a few harmonic bands: fundamental, second harmonic and third harmonic bands. Designing the shaping filters which cover the whole bandwidth from DC to the third harmonic band is very difficult due to the well-known gain-bandwidth trade-off. This difficulty limits current available designs to either focusing on low operating frequency or only the fundamental band, or using discrete components $[4,16,23,25,50,55-58]$. Realize that the output appears only at a few frequency bands, we choose to design the shaping filters for only those frequency bands in stead of the whole bandwidth to relieve the difficulty.

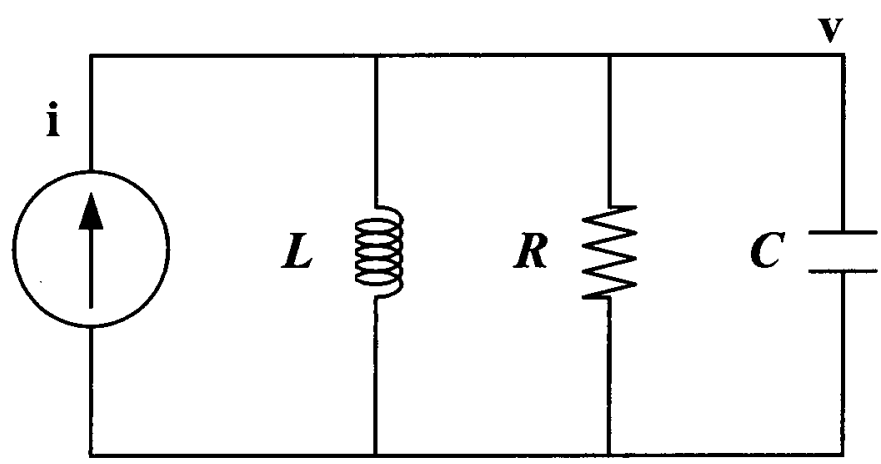

Figure 3.6: LC tank circuit.

Figure 3.6 is a LC tank circuit driven by a current source. The voltage applied to the tank circuit can be obtained as

$$
V(s)=\frac{a s}{s^{2}+\beta s+\omega_{0}^{2}}
$$

where

$$
\begin{gathered}
\omega_{0}=\sqrt{\frac{1}{L C}} \\
\beta=\frac{1}{R C}
\end{gathered}
$$

and

$$
a=\frac{i}{C}
$$


$\omega_{0}$ is the center frequency and $\beta$ is the $3 \mathrm{~dB}$ bandwidth. The phase of voltage $v$ is

$$
\phi=\operatorname{Tan}^{-1}\left(\frac{\omega_{0}^{2}-\omega^{2}}{\beta \omega}\right) \approx-\frac{2\left(\omega-\omega_{0}\right)}{\beta} .
$$

Group delay around the center frequency is approximately

$$
\frac{\partial \phi}{\partial \omega} \approx-\frac{2}{\beta}
$$

Therefore, regardless of the center frequency, tank circuits with equal $3 \mathrm{~dB}$ bandwidths have approximately the same amount of group delay in the passband.
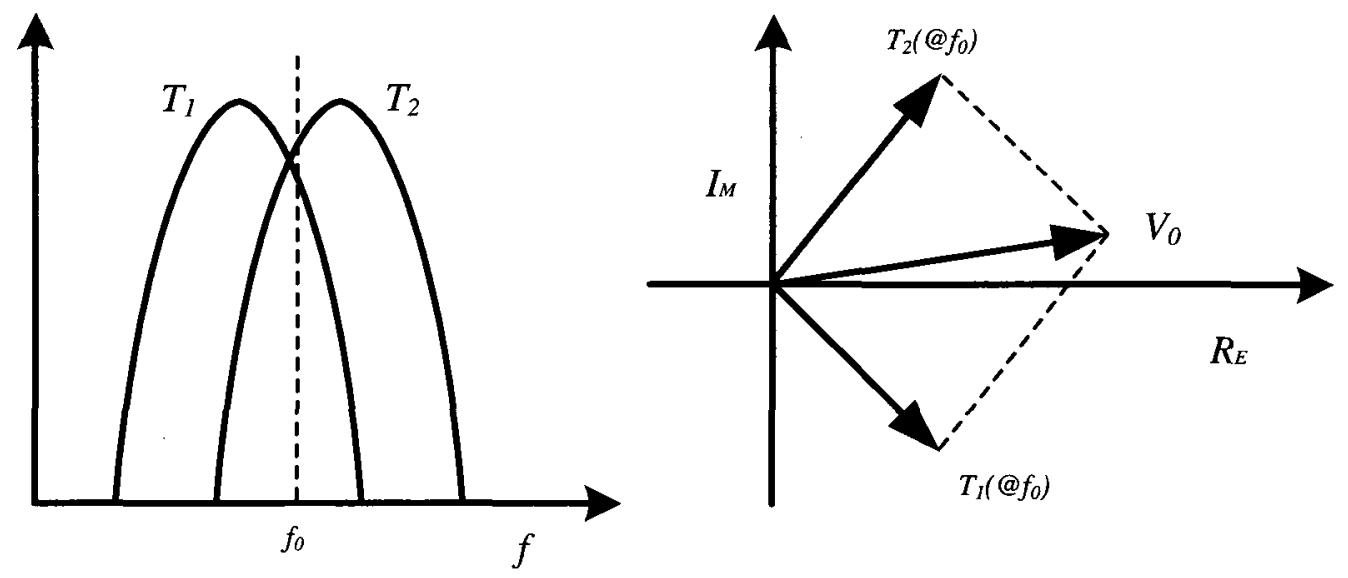

Figure 3.7: Amplitude and phase interpolation using two tank circuits.

Figure 3.7 demonstrates the concept of amplitude and phase interpolation using two tank circuits. The impedances at the opposite side of the center frequency of a tank circuit have approximately 90 degree phase difference near the $-3 \mathrm{~dB}$ band edges. An arbitrary vector can be represented as a linear combination of two given linear-independent vectors:

$$
\vec{V}_{0}=i_{1} \times \vec{T}_{1}+i_{2} \times \vec{T}_{2}
$$

at a given frequency, the complex value of the shaping filter can be obtained by a linear combination of the output voltages from two tank circuits. By varying the driving current $i_{1}$ and $i_{2}$, arbitrary complex value can be achieved. Figure 3.8 illustrates the proposed 


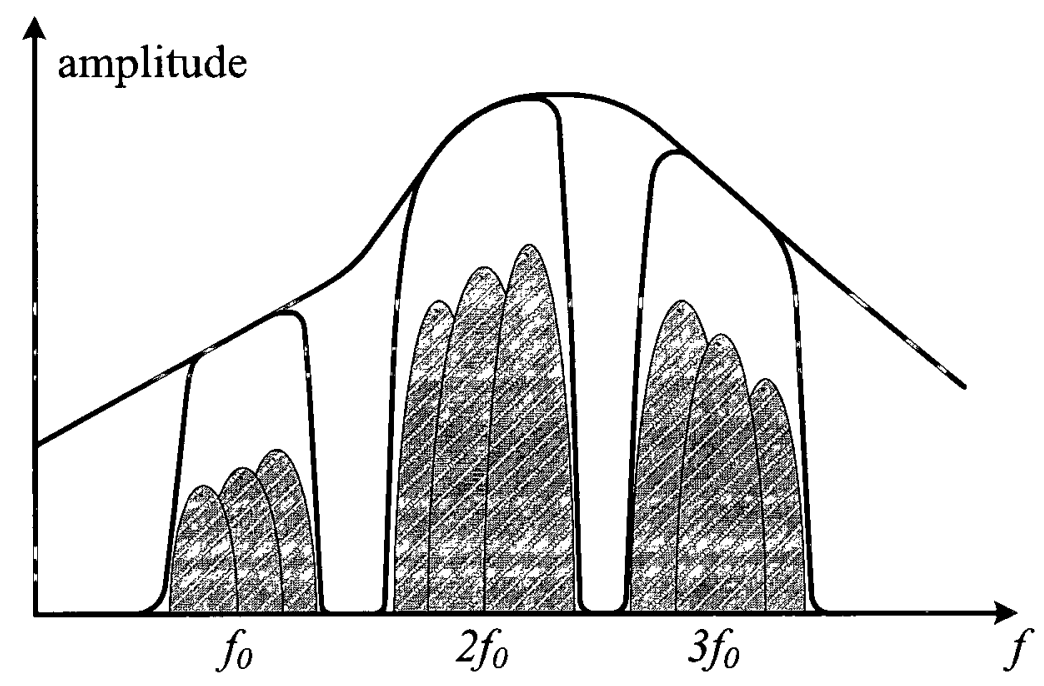

Figure 3.8: Multi-tank approach for frequency profile shaping.

multiple tank approach. Under this approach, the amplitude and the phase of the frequency profile shaping functions $G_{1}(s), G_{2}(s)$ and $G_{3}(s)$ are approximated using multiple tank circuits at the frequency band of interest. While two tank circuit are able to match the shaping filter at one frequency point, multiple frequency points can be matched with more tank circuit and the resulted frequency profile will be naturally interpolated to the desired continuous shape. The capability of tuning the shape of the frequency profile is provided by adjusting the driving current. Because of this tunability and the bandpass feature of the tank circuit, coarse squaring and cubing circuits can be used as distortion generators.

\subsection{Tank Gain Calculation}

Figure 3.9 depicts the predistorter model using multiple tank approach, where the frequency profile shaping files are replaced by the weighted sum of tank circuit impedances and $X, X^{2}$, and $X^{3}$ components are replaced by coarse linear, square and cubic components $D_{1}, D_{2}$, and $D_{3}$. 


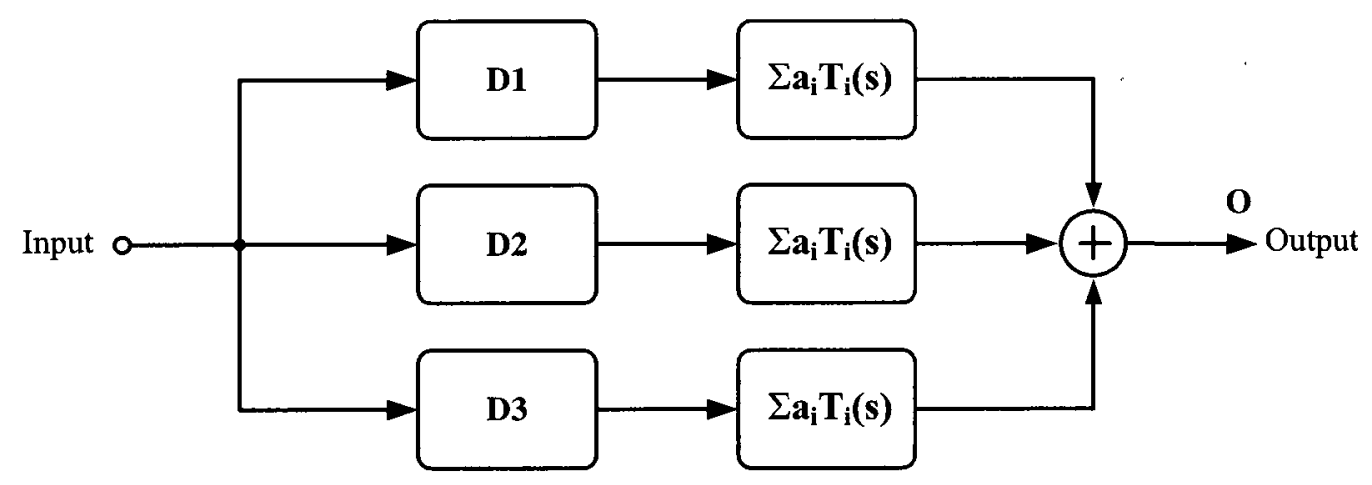

Figure 3.9: Predistorter model using multi-tank approach.

The multi-tank expansion of the predistorter model can be formulated as:

$$
\vec{P}(s)=\vec{D}_{1} \sum_{i=1}^{n} a_{i} T(s)_{i}+\vec{D}_{2} \sum_{i=n+1}^{n+l} a_{i} T(s)_{i}+\vec{D}_{3} \sum_{i=n+l+1}^{n+l+m} a_{i} T(s)_{i}
$$

where $a_{i}$ represents the gain (or weight) applied to each tank circuit, $n, m$, and $l$ are the number of the tank circuits used in $X, X^{2}$, and $X^{3}$ paths respectively, and

$$
\begin{aligned}
& \vec{D}_{1}=c_{1} \vec{I}+c_{2} \vec{J}+c_{3} \vec{K} \\
& \vec{D}_{2}=c_{4} \vec{I}+c_{5} \vec{J}+c_{6} \vec{K} \\
& \vec{D}_{3}=c_{7} \vec{I}+c_{8} \vec{J}+c_{9} \vec{K} .
\end{aligned}
$$

They represent coarse $X, X^{2}$, and $X^{3}$ generators respectively, in Taylor series form

$$
\begin{aligned}
& D_{1}(x)=c_{1} x+c_{2} x^{2}+c_{3} x^{3} \\
& D_{2}(x)=c_{4} x+c_{5} x^{2}+c_{6} x^{3} \\
& D_{3}(x)=c_{7} x+c_{8} x^{2}+c_{9} x^{3} .
\end{aligned}
$$

\subsubsection{Based on Optimization}

In order to use the expansion function (3.3.1) to approximate the predistorter model $L(s)$ (3.1.3), the mismatch (or the difference) between $L(s)$ and $P(s)$ needs to be minimized for the frequency band of interest $(\Omega)$. The mismatch is defined as, 


$$
\begin{aligned}
& F\left(a_{1}, a_{2}, \ldots, a_{n+l+m}\right)=\int_{\Omega}|\vec{P}(s)-\vec{L}(s)|^{2} d \Omega \\
& =\int_{\Omega} \mid\left(c_{1} \vec{I}+c_{2} \vec{J}+c_{3} \vec{K}\right) \sum_{i=1}^{n} a_{i} T(s)_{i}+\left(c_{4} \vec{I}+c_{5} \vec{J}+c_{6} \vec{K}\right) \sum_{i=n+1}^{n+l} a_{i} T(s)_{i} \\
& +\left(c_{7} \vec{I}+c_{8} \vec{J}+c_{9} \vec{K}\right) \sum_{i=n+l+1}^{n+l+m} a_{i} T(s)_{i}-\left.\left(G_{1}(s) \vec{I}+G_{2}(s) \vec{J}+G_{3}(s) \vec{K}\right) e^{-s \Delta t}\right|^{2} d \Omega .
\end{aligned}
$$

The frequency bands of interest are different for $X, X^{2}$ and $X^{3}$ functions, we have

$$
\begin{aligned}
& F\left(a_{1}, a_{2}, \ldots, a_{n+l+m}\right)= \\
& \int_{\Omega 1}\left|c_{1} \sum_{i=1}^{n} a_{i} T(s)_{i}+c_{4} \sum_{i=n+1}^{n+l} a_{i} T(s)_{i}+c_{7} \sum_{i=n+l+1}^{n+l+m} a_{i} T(s)_{i}-G_{1}(s) e^{-s \Delta t}\right|^{2} d \Omega_{1}+ \\
& \int_{\Omega 2}\left|c_{2} \sum_{i=1}^{n} a_{i} T(s)_{i}+c_{5} \sum_{i=n+1}^{n+l} a_{i} T(s)_{i}+c_{8} \sum_{\substack{i=n+l+1 \\
n+l+m}}^{n+n} T(s)_{i}-G_{2}(s) e^{-s \Delta t}\right|^{2} d \Omega_{2}+ \\
& \int_{\Omega 3}\left|c_{3} \sum_{i=1}^{n} a_{i} T(s)_{i}+c_{6} \sum_{i=n+1}^{n+l} a_{i} T(s)_{i}+c_{9} \sum_{i=n+l+1}^{n+l+m} a_{i} T(s)_{i}-G_{3}(s) e^{-s \Delta t}\right|^{2} d \Omega_{3} .
\end{aligned}
$$

After rearrangement,

$$
\begin{aligned}
& F\left(a_{1}, a_{2}, \ldots, a_{n+l+m}\right) \\
& =\sum_{i=1}^{n+l+m} \sum_{j=1}^{n+l+m} a_{i} a_{j}\left(\begin{array}{c}
c_{x} c_{o} \int_{\Omega 1}\left(T(s)_{i} T(s)_{j}^{*}+T(s)_{i}^{*} T(s)_{j}\right) d \Omega_{1}+ \\
c_{y} c_{p} \int_{\Omega 2}\left(T(s)_{i} T(s)_{j}^{*}+T(s)_{i}^{*} T(s)_{j}\right) d \Omega_{2}+ \\
c_{z} c_{q} \int_{\Omega 3}\left(T(s)_{i} T(s)_{j}^{*}+T(s)_{i}^{*} T(s)_{j}\right) d \Omega_{3}
\end{array}\right) \\
& -\sum_{i=1}^{n+l+m} a_{i}\left(\begin{array}{c}
\int_{\Omega 1}\left(c_{x} G_{1}(s) e^{-s \Delta t} T(s)_{i}^{*}+T(s)_{i} c_{x} G_{1}(s)^{*} e^{s \Delta t}\right) d \Omega_{1}+ \\
\int_{\Omega 2}\left(c_{y} G_{2}(s) e^{-s \Delta t} T(s)_{i}^{*}+T(s)_{i} c_{y} G_{2}(s)^{*} e^{s \Delta t}\right) d \Omega_{2}+ \\
\int_{\Omega 3}\left(c_{z} G_{3}(s) e^{-s \Delta t} T(s)_{i}^{*}+T(s)_{i} c_{z} G_{3}(s)^{*} e^{s \Delta t}\right) d \Omega_{3}
\end{array}\right) \\
& +\int_{\Omega 1}\left|G_{1}(s)\right|^{2} d \Omega_{1}+\int_{\Omega 2}\left|G_{2}(s)\right|^{2} d \Omega_{2}+\int_{\Omega 3}\left|G_{3}(s)\right|^{2} d \Omega_{3}
\end{aligned}
$$

where the subscripts of $c$ are

$$
\begin{array}{ll}
x=1, y=2, z=3 & (i \in 1, . ., n) \\
x=4, y=5, z=6 & (i \in n+1, . ., n+l) \\
x=7, y=8, z=9 & (i \in n+l+1, . ., n+l+m) \\
o=1, p=2, q=3 & (j \in 1, . ., n) \\
o=4, p=5, q=6 & (j \in n+1, . ., n+l) \\
o=7, p=8, q=9 & (j \in n+l+1, . ., n+l+m) .
\end{array}
$$


Minimizing the difference by setting its derivatives with respect to $a_{i}$ equal to zero,

$$
\frac{\partial F}{\partial a_{i}}=0 ; \quad(i=1,2, . ., n+l+m)
$$

and expressing it in a system form, we have

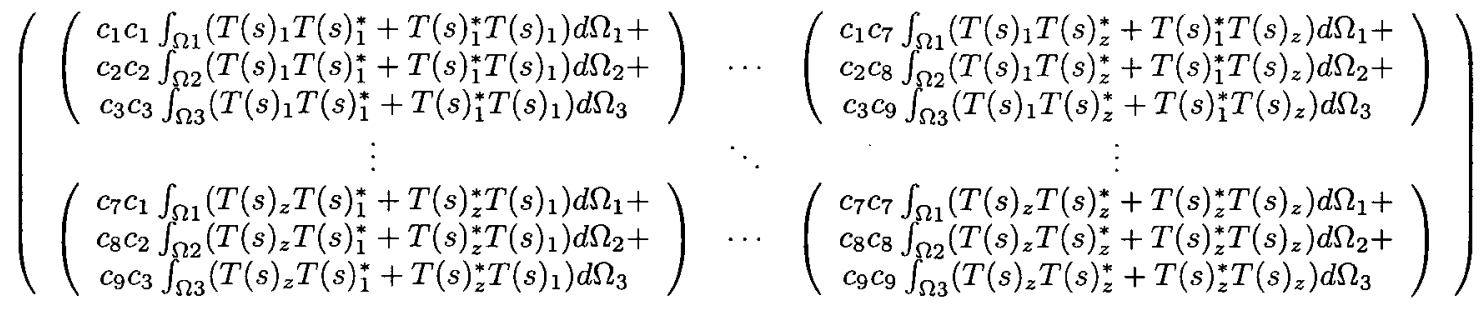

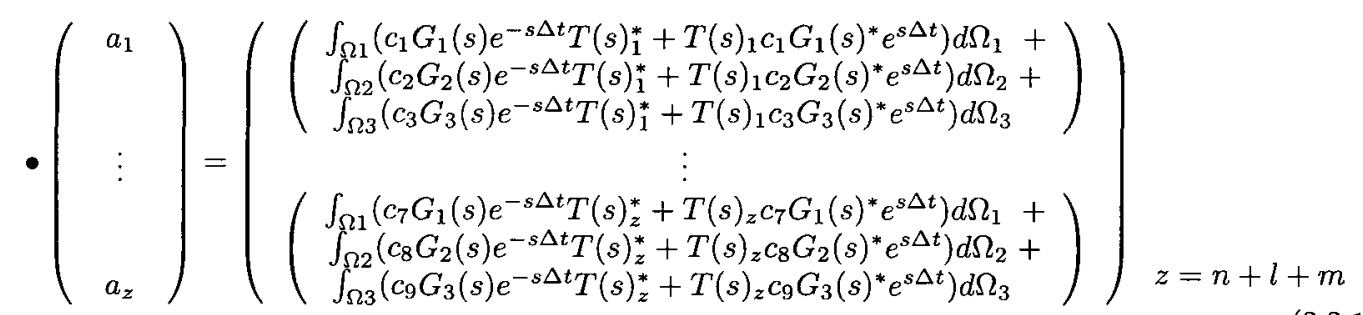

or

$$
S \vec{a}=\vec{b}
$$

The weighting coefficients $a_{i}$ can be obtained by solving the $(n+l+m) \times(n+l+m)$ matrix S:

$$
\vec{a}=S^{-1} \vec{b}
$$

\subsubsection{Based on Point Matching}

The optimization method of calculating the weighting coefficients provides a smooth interpolation within the frequency range of interest. However, in reality, output will be sampled only at a few frequency points to detect distortions. It would not be possible or efficient to do the integration required by the optimization approach. In circuit calibration, the weighing coefficient of each tank circuit can be obtained by matching to the required frequency profile only at several chosen frequency points $\left(\omega_{1}, \omega_{2}, \ldots, \omega_{(n / 2+l / 2+m / 2)}\right)$, 


$$
\begin{array}{l|l}
\operatorname{Re}[P(\omega)-L(\omega)]=0 & \\
\operatorname{Im}[P(\omega)-L(\omega)]=0 & \omega=\left(\omega_{1}, \ldots \omega_{(n / 2+l / 2+m / 2)}\right) .
\end{array}
$$

After expansion, we have

$$
\begin{array}{ll|l}
c_{1} \sum_{i=1}^{n} a_{i} \operatorname{Re}\left[T(\omega)_{i}\right]+c_{4} & \sum_{i=n+1}^{n+l} a_{i} \operatorname{Re}\left[T(\omega)_{i}\right]+c_{7} \sum_{i=n+l+1}^{n+l+m} a_{i} \operatorname{Re}\left[T(\omega)_{i}\right]-\operatorname{Re}\left[G_{1}(\omega) e^{-i \omega \Delta t}\right]=0 & \\
c_{1} \sum_{i=1}^{n} a_{i} \operatorname{Im}\left[T(\omega)_{i}\right]+c_{4} \sum_{i=n+1}^{n+l} a_{i} \operatorname{Im}\left[T(\omega)_{i}\right]+c_{7} \sum_{i=n+l+1}^{n+l+m} a_{i} \operatorname{Im}\left[T(\omega)_{i}\right]-\operatorname{Im}\left[G_{1}(\omega) e^{-i \omega \Delta t}\right]=0 & \omega_{1} . \omega_{(n / 2)} \\
c_{2} \sum_{i=1}^{n} a_{i} \operatorname{Re}\left[T(\omega)_{i}\right]+c_{5} \sum_{i=n+1}^{n+l} a_{i} \operatorname{Re}\left[T(\omega)_{i}\right]+c_{8} \sum_{i=n+l+1}^{n+l+m} a_{i} \operatorname{Re}\left[T(\omega)_{i}\right]-\operatorname{Re}\left[G_{2}(\omega) e^{-i \omega \Delta t}\right]=0 & \omega_{(n / 2+1) \cdot .} \\
c_{2} \sum_{i=1}^{n} a_{i} \operatorname{Im}\left[T(\omega)_{i}\right]+c_{5} \sum_{i=n+1}^{n+l} a_{i} \operatorname{Im}\left[T(\omega)_{i}\right]+c_{8} \sum_{i=n+l+1}^{n+l+m} a_{i} \operatorname{Im}\left[T(\omega)_{i}\right]-\operatorname{Im}\left[G_{2}(\omega) e^{-i \omega \Delta t}\right]=0 & \omega_{((n+l) / 2)} \\
c_{3} \sum_{i=1}^{n} a_{i} \operatorname{Re}\left[T(\omega)_{i}\right]+c_{6} \sum_{i=n+1}^{n+l} a_{i} \operatorname{Re}\left[T(\omega)_{i}\right]+c_{9} \sum_{i=n+l+1}^{n+l+m} a_{i} \operatorname{Re}\left[T(\omega)_{i}\right]-\operatorname{Re}\left[G_{3}(\omega) e^{-i \omega \Delta t}\right]=0 & \omega_{((n+l) / 2+1) \cdot \cdot} \\
c_{3} \sum_{i=1}^{n} a_{i} \operatorname{Im}\left[T(\omega)_{i}\right]+c_{6} \sum_{i=n+1}^{n+l} a_{i} \operatorname{Im}\left[T(\omega)_{i}\right]+c_{9} \sum_{i=n+l+1}^{n+l+m} a_{i} \operatorname{Im}\left[T(\omega)_{i}\right]-\operatorname{Im}\left[G_{9}(\omega) e^{-i \omega \Delta t}\right]=0 & \omega_{((n+l+m) / 2)}
\end{array}
$$

The number of tank circuits for $X, X^{2}$, and $X^{3}$ paths should be even numbers in order to let both real part or imaginary part equal to zero at the frequency points selected. Depending on the number of the frequency points, the above equations corresponds to a $(n+l+m) \times(n+l+m)$ matrix. The coefficients $a_{i}$ can be obtained by solving the linear matrix.

\subsection{Mismatch and Distortion}

\subsubsection{Tank Circuit Parameters Optimization}

In order to obtain a good interpolation and to reduce the mismatch between the desired frequency profile and the one interpolated by the tank circuits, the number and the parameters of the tank circuits need to be optimized. For equal time delays, the tank circuits have the same bandwidth $\beta$. The center frequencies are equally spaced by $d \omega$. The tank circuit impedances can be represented by the second order bandpass transfer function:

$$
T_{n}(s)=\frac{a_{n} s}{s^{2}+\beta s+\left(\omega_{0}+n d \omega\right)^{2}}
$$


where $a_{n}$ is the weighting coefficient. The mismatch is defined as:

$$
E_{m i s}=\left(\frac{\int_{\Omega}|P(s)-L(s)|^{2} d \Omega}{\int_{\Omega} d \Omega}\right)^{1 / 2}=f(\beta, d \omega) .
$$

Different values of bandwidth $\beta$ and spacing $d \omega$ (illustrated in Figure 3.10) can be exercised to minimize the mismatch, hence to produce a smooth interpolation.

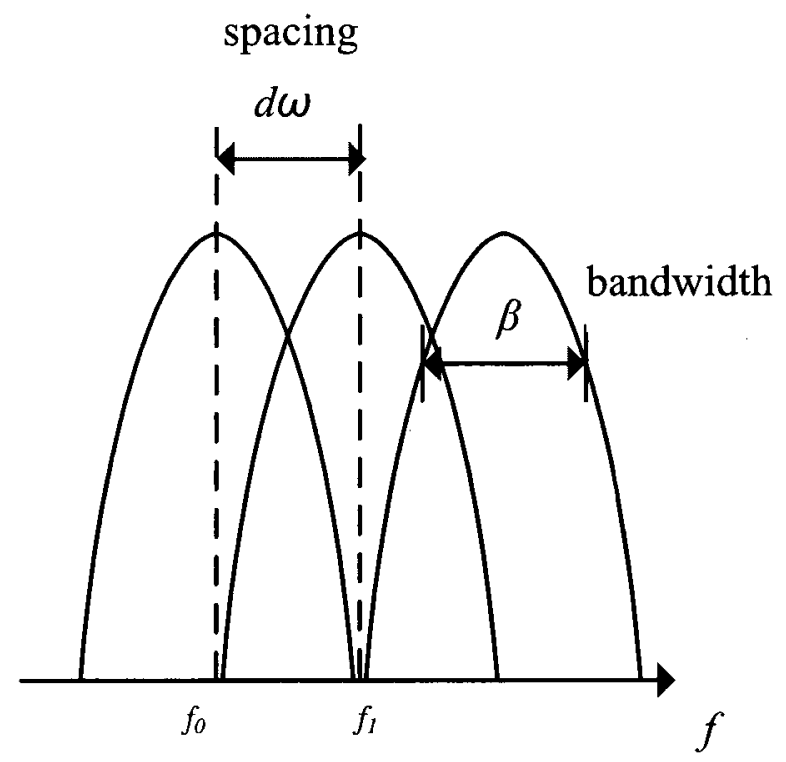

Figure 3.10: Illustration of bandwidth and spacing.

\subsection{2 $2^{\text {nd }}$ Order Distortion}

Consider the second order Volterra kernel in equation (3.1.9), and rewrite here:

$$
Q_{2}\left(s_{1}, s_{2}\right)=H_{2}\left(s_{1}, s_{2}\right) F_{1}\left(s_{1}+s_{2}\right)+H_{1}\left(s_{1}\right) H_{1}\left(s_{2}\right) F_{1}\left(s_{1}, s_{2}\right)
$$

where

$$
F_{1}\left(s_{1}+s_{2}\right)=1 / G_{1}\left(s_{1}+s_{2}\right)
$$

The mismatch induced $2^{\text {nd }}$ order distortion can be obtained as:

$$
D 2_{m i s}\left(s_{1}, s_{2}\right)=\frac{\left[P\left(s_{1}+s_{2}\right)-L\left(s_{1}+s_{2}\right)\right]_{X^{2} p a t h}}{G_{1}\left(s_{1}+s_{2}\right)} .
$$


Specifically, the mismatch induced $2^{\text {nd }}$ order harmonic distortion is

$$
H D_{2}\left(f_{0}\right)=\frac{R F_{i n}^{2}}{2} \frac{\left[P\left(2 f_{0}\right)-L\left(2 f_{0}\right)\right]_{X^{2} p a t h}}{G_{1}\left(2 f_{0}\right)}
$$

The mismatch induced $2^{\text {nd }}$ order intermodulation distortion would be:

$$
I M_{2}\left(f_{1} \pm f_{2}\right)=R F_{i n}^{2} \frac{\left.\left[P\left(f_{1} \pm f_{2}\right)-L\left(f_{1} \pm f_{2}\right)\right]\right|_{X^{2} \text { path }}}{G_{1}\left(f_{1} \pm f_{2}\right)} .
$$

\subsection{3 $\quad 3^{\text {rd }}$ Order Distortion}

Considering the third order Volterra kernel in equation (3.1.9) and (3.4.8),

$$
\begin{aligned}
Q_{3}\left(s_{1}, s_{2}, s_{3}\right)= & H_{3}\left(s_{1}, s_{2}, s_{3}\right) F_{1}\left(s_{1}+s_{2}+s_{3}\right)+H_{1}\left(s_{1}\right) H_{1}\left(s_{2}\right) H_{1}\left(s_{3}\right) F_{3}\left(s_{1}, s_{2}, s_{3}\right) \\
& +\frac{2}{3} H_{1}\left(s_{1}\right) H_{2}\left(s_{2}, s_{3}\right) F_{2}\left(s_{1}, s_{2}+s_{3}\right) \\
& +\frac{2}{3} H_{1}\left(s_{2}\right) H_{2}\left(s_{1}, s_{3}\right) F_{2}\left(s_{2}, s_{1}+s_{3}\right) \\
& +\frac{2}{3} H_{1}\left(s_{3}\right) H_{2}\left(s_{1}, s_{2}\right) F_{2}\left(s_{3}, s_{1}+s_{2}\right),
\end{aligned}
$$

the mismatch induced $3^{\text {rd }}$ order distortion can be obtained as

$$
\begin{aligned}
D 3_{m i s}\left(s_{1}, s_{2}, s_{3}\right)= & {\left[P\left(s_{1}+s_{2}+s_{3}\right)-L\left(s_{1}+s_{2}+s_{3}\right)\right]_{X^{3} p a t h} \times F_{1}\left(s_{1}+s_{2}+s_{3}\right) } \\
& +\frac{2}{3} H_{1}\left(s_{1}\right)\left[P\left(s_{2}+s_{3}\right)-L\left(s_{2}+s_{3}\right)\right]_{X^{2} p a t h} \times F_{2}\left(s_{1}, s_{2}+s_{3}\right) \\
& +\frac{2}{3} H_{1}\left(s_{2}\right)\left[P\left(s_{1}+s_{3}\right)-L\left(s_{1}+s_{3}\right)\right]_{X^{2} p a t h} \times F_{2}\left(s_{2}, s_{1}+s_{3}\right) \\
& +\frac{2}{3} H_{1}\left(s_{3}\right)\left[P\left(s_{1}+s_{2}\right)-L\left(s_{1}+s_{2}\right)\right]_{X^{2} p a t h} \times F_{2}\left(s_{3}, s_{1}+s_{2}\right) .
\end{aligned}
$$

Specifically, the mismatch induced $3^{\text {rd }}$ order harmonic distortion is

$$
\begin{aligned}
H D_{3}\left(f_{0}\right)= & \frac{R F_{i n}^{3}}{4}\left(\left[P\left(3 f_{0}\right)-L\left(3 f_{0}\right)\right]_{X^{3} \text { path }} \times F_{1}\left(3 f_{0}\right)\right. \\
& \left.+\left.2 H_{1}\left(f_{0}\right)\left[P\left(2 f_{0}\right)-L\left(2 f_{0}\right)\right]\right|_{X^{2} \text { path }} \times F_{2}\left(f_{0}, 2 f_{0}\right)\right) .
\end{aligned}
$$

The mismatch induced $3^{\text {rd }}$ order intermodulation distortion is

$$
\begin{aligned}
I M_{3}\left(2 f_{1} \pm f_{2}\right)= & \frac{3 R F_{i n}^{3}}{4}\left(\left[P\left(2 f_{1} \pm f_{2}\right)-L\left(2 f_{1} \pm f_{2}\right)\right]_{X^{3} \text { path }} \times F_{1}\left(2 f_{1} \pm f_{2}\right)\right. \\
& +\left.\frac{4}{3} H_{1}\left(f_{1}\right)\left[P\left(f_{1} \pm f_{2}\right)-L\left(f_{1} \pm f_{2}\right)\right]\right|_{X^{2} \text { path }} \times F_{2}\left(f_{1}, f_{1} \pm f_{2}\right) \\
& \left.+\left.\frac{2}{3} H_{1}\left( \pm f_{2}\right)\left[P\left(2 f_{1}\right)-L\left(2 f_{1}\right)\right]\right|_{X^{2} \text { path }} \times F_{2}\left( \pm f_{2}, 2 f_{1}\right)\right) .
\end{aligned}
$$




\subsection{Sensitivity, Resolution, and Tuning Range}

The derivative of the distortion with respect to the weight of each tank circuit is

$$
D E R(f)=\frac{\partial D(f)}{\partial a_{i}}
$$

The derivative of $\mathrm{HD}_{2}$ to the weight of each tank circuit for $X^{2}$ path is

$$
D E R_{H D 2}(f)=\frac{\partial\left(P(2 f) / G_{1}(2 f)\right)}{\partial a_{i}}=\frac{c_{5} T(2 f)_{i}}{G_{1}(2 f)} .
$$

The derivative of $I M_{2}$ to the weight of each tank circuit for $X^{2}$ path is

$$
D E R_{I M 2}\left(f_{1} \pm f_{2}\right)=\frac{\partial\left(P\left(f_{1} \pm f_{2}\right) / G_{1}\left(f_{1} \pm f_{2}\right)\right)}{\partial a_{i}}=\frac{c_{5} T\left(f_{1} \pm f_{2}\right)_{i}}{G_{1}\left(f_{1} \pm f_{2}\right)} .
$$

The derivative of $H D_{3}$ to the weight of each tank circuit for $X^{3}$ path is

$$
D E R_{H D 3}(f)=\frac{\partial\left(P(3 f) / G_{1}(3 f)\right)}{\partial a_{i}}=\frac{c_{9} T(3 f)_{i}}{G_{1}(3 f)} .
$$

The derivative of $I M_{3}$ to the weight of each tank circuit for $X^{3}$ path is

$$
D E R_{I M 3}\left(2 f_{1} \pm f_{2}\right)=\frac{\partial\left(P\left(2 f_{1} \pm f_{2}\right) / G_{1}\left(2 f_{1} \pm f_{2}\right)\right)}{\partial a_{i}}=\frac{c_{9} T\left(2 f_{1} \pm f_{2}\right)_{i}}{G_{1}\left(2 f_{1} \pm f_{2}\right)}
$$

The conventional sensitivity is defined as

$$
S(f)=\frac{\partial D(f) / D(f)}{\partial a_{i} / a_{i}}=\frac{\partial D(f)}{\partial a_{i}} \frac{a_{i}}{D(f)}=D E R \frac{a_{i}}{D(f)}
$$

where $D(f)$ is the mismatch introduced distortion plus the residual distortion which can not be compensated by a third order predistorter.

While the specific application defines the minimum distortion $\left(D_{\min }\right)$, the resolution of the digitized weighting coefficients $\left(R_{a i}\right)$ defines the minimum controllable distortion. Therefore:

$$
D E R \times R_{a i} \leq D_{\min }
$$

The least significant bit (LSB) of the control signal corresponds to the gain resolution,

$$
R_{a i} \leq D_{\min } / D E R
$$


should be satisfied. The variation of semiconductor laser diode due to manufacturer, temperature and aging changes the laser parameters and hence the weighting coefficient of each tank circuit. To account for those effects, tunability should be provided. This requires the weights to be adjustable for certain range. For a wide tuning range, the maximum allowed inherited laser distortion defines the most significant bit (MSB) of the gain control signal. Therefore, a $30 \mathrm{~dB}(\approx 31)$ distortion reduction requires 5 bits of resolution $\left(2^{5}=32\right)$. This

gives the lower bound estimate, considering strong interactions among tank circuits for the same frequency band, more digits are required.

\subsection{Noise Figure Consideration}

Considering $1 \mathrm{MHz}$ as the signal detection bandwidth, in order to detect the distortion level of $-80 \mathrm{dBc}$, the maximum noise power from the laser driver can be derived as follows:

$$
\begin{gathered}
\frac{I_{s}}{I_{L}}=\frac{I_{s} \times G_{L}}{I_{L} \times G_{L}}=\frac{P_{s}}{P_{L}}=10^{-80 / 20} \\
I_{s}=\frac{P_{L}}{G_{L}} \times 10^{-4.0}=\frac{10^{-0.6-3}}{0.05} \times 10^{-4.0}=0.5 \mu \mathrm{A}
\end{gathered}
$$

where $I_{s}$ is the noise current and $I_{L}$ the signal current. $G_{L}$ is the laser gain, which is about $0.05 \mathrm{Watt} / \mathrm{A}$. Assume that the maximum laser output power $P_{L}$ (used for distortion detection) is $-6 \mathrm{dBm}$.

$$
P_{I s}=\frac{1}{2}\left(I_{s}\right)^{2} R=\frac{1}{2}(0.5 \mu A)^{2} 25 \Omega=-85 d B m
$$

Assuming conjugate matching at the input, the noise power at the input is equal to $k T$ $(-174 \mathrm{dBm} / \mathrm{Hz})$ [59]. So the maximum noise power $\left(P_{I s}\right)$ from laser diver output:

$$
P_{I s}=-174 d B m / H z+N F+G_{P}+10 \log B
$$

Assuming that the predistorter gain $G_{P}$ is $0 \mathrm{~dB}$ and the detection bandwidth $B$ is $1 \mathrm{MHz}$, we have

$$
N F=P_{I s}-\left(-174 d B m / H z+10 \log B+G_{P}\right)=29 d B .
$$


Therefore, the noise figure of the predistortion circuit should be less than $29 \mathrm{~dB}$, which is easy to achieve. Note that relative intensity noise of the laser itself is neglected since it is much less than $-80 \mathrm{dBc}$.

\subsection{Summary}

In this chapter, a multiple tank technique has been introduced and described. The design problem is described as both an optimization problem and a point matching problem. The former is to be used in the design stage to search the optimal parameters which provide smooth interpolation and the latter is a theoretical support for the calibration algorithm and measurement setups described in Chapter 6 . The effects of the mismatch between the multi-tank approximation and the ideal predistorter model are analyzed and formulated. At last, the noise figure requirement is estimated. 


\section{Chapter 4}

\section{Predistortion Circuit Design in CMOS}

This chapter will detail the design of a laser predistorter using the multiple tank technique described in previous chapter. Standard $0.18 \mu \mathrm{m}$ CMOS technology is chosen to demonstrate the feasibility of this technique. First, the design of each circuit building block is described and discussed. Previous chapter introduced high level topology of the predistorter design. This chapter details how to break down the high level predistorter parameters into circuit design parameters. A CMOS predistorter is designed and the tank circuit parameters are obtained by the optimization technique described in previous chapter. Finally, the overall simulation results will be given and the design methodology will be summarized.

\subsection{Circuit Building Blocks}

According to the predistorter model illustrated in Figure 3.9 in Chapter 3, circuit building blocks of the predistorter include: 1) the linear path which will be simply a through connection using a transmission-line structure or passive filters to reduce the length; 2) distortion generators - $X^{2}$ and $X^{3}$ components; 3) tank circuits with a tunable gain to adjust the weighting coefficients 4 ) and a summing circuit to combine the outputs from the linear, $X^{2}$, and $X^{3}$ paths. 


\subsection{1 $\quad X^{2}$ Component}

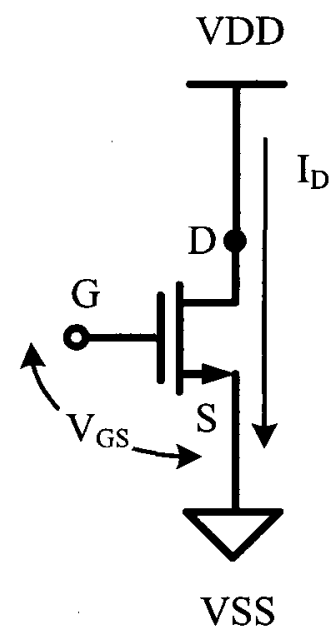

Figure 4.1: NMOS device.

Figure 4.1 shows a MOS device (NMOS). $V_{G S}$ is the gate to source voltage and $I_{D}$ is the drain current. In saturation region, the input-output characteristic of a MOS device can be approximated by a square-law relationship [60], as follows:

$$
I_{D}=\frac{\mu C_{o x} W}{2 L}\left(V_{G S}-V t h\right)^{2}
$$

where $V_{t h}$ is the threshold voltage of the MOS device. Replacing $V_{G S}$ by an combination of ac signal $v_{i}$ and dc bias voltage $V_{b}$, the output drain current is given by

$$
I_{D}=K\left(V_{b}-V t h\right)^{2}+K v_{i}^{2}+2 K\left(V_{b}-V t h\right) v_{i}
$$

where

$$
K=\frac{\mu C_{o x} W}{2 L}
$$

is the coefficient of the square term. As the square-law is only an approximation, the exact V-I characteristic was obtained by simulation. The simulated input-output (gain-source voltage versus drain current) characteristic of an NMOS transistor is shown in Figure 4.2. At dc bias voltage $V_{b}$, the V-I curve can be represented by a Taylor series expansion and 


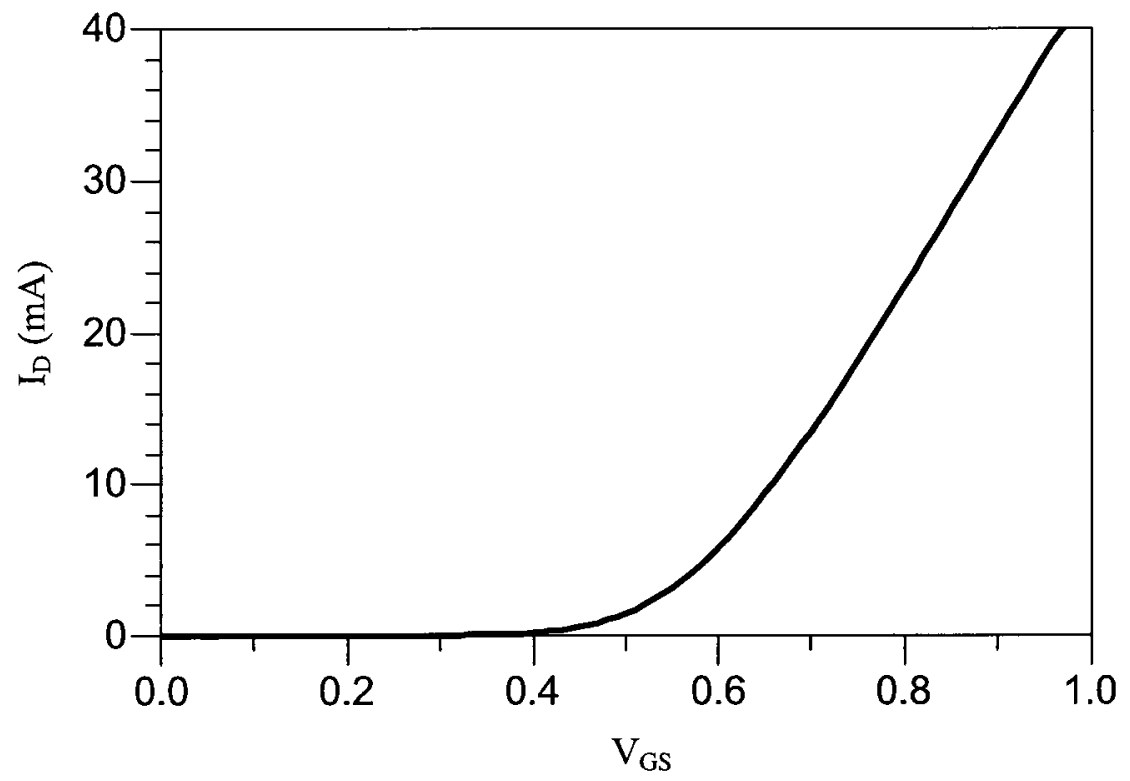

Figure 4.2: Simulated input-output (V-I) characteristic of an nmos transistor (W=240 $\mu \mathrm{m}$, $\mathrm{L}=0.18 \mu \mathrm{m}$ and 24 fingers).

written as,

$$
I_{D}=c_{0}+c_{1} v_{i}+c_{2} v_{i}^{2}+c_{3} v_{i}^{3}+\ldots
$$

where

$$
\begin{aligned}
& c_{0}=\left.I_{D}\right|_{V_{G S}=V_{b}} \\
& c_{1}=\left.\left(I_{D}\right)^{\prime}\right|_{V_{G S}=V_{b}} \\
& c_{2}=\left.\frac{1}{2}\left(I_{D}\right)^{\prime \prime}\right|_{V_{G S}=V_{b}} \\
& c_{3}=\left.\frac{1}{6}\left(I_{D}\right)^{\prime \prime \prime}\right|_{V_{G S}=V_{b}} .
\end{aligned}
$$

Figure 4.3 plots the linear, square and cubic terms obtained by applying (4.1.5) to the $\mathrm{I}-\mathrm{V}$ curve in Figure 4.2. The electron mobility $\mu$ drops while increasing the bias voltage $V_{b}$ due to transverse field effects. In short channel device, as the channel length becomes smaller, the longitudinal electric field increases and the carrier drift velocity approaches the saturation value, which further reduces the effective mobility. Thus, increasing $V_{b}$ reduces the square term due to the decrease of the electron mobility. While decreasing $V_{b}$ gradually turns off the transistor, decreasing $V_{b}$ also reduces the square term. Therefore, the coefficient of the square term has a "Bell" shape when plotted versus the bias voltage, 
as shown in Figure 4.3. From this figure, it can be decided that the dc bias for squaring circuit should be where $c_{2}$ is the maximum and $c_{3}$ is zero.

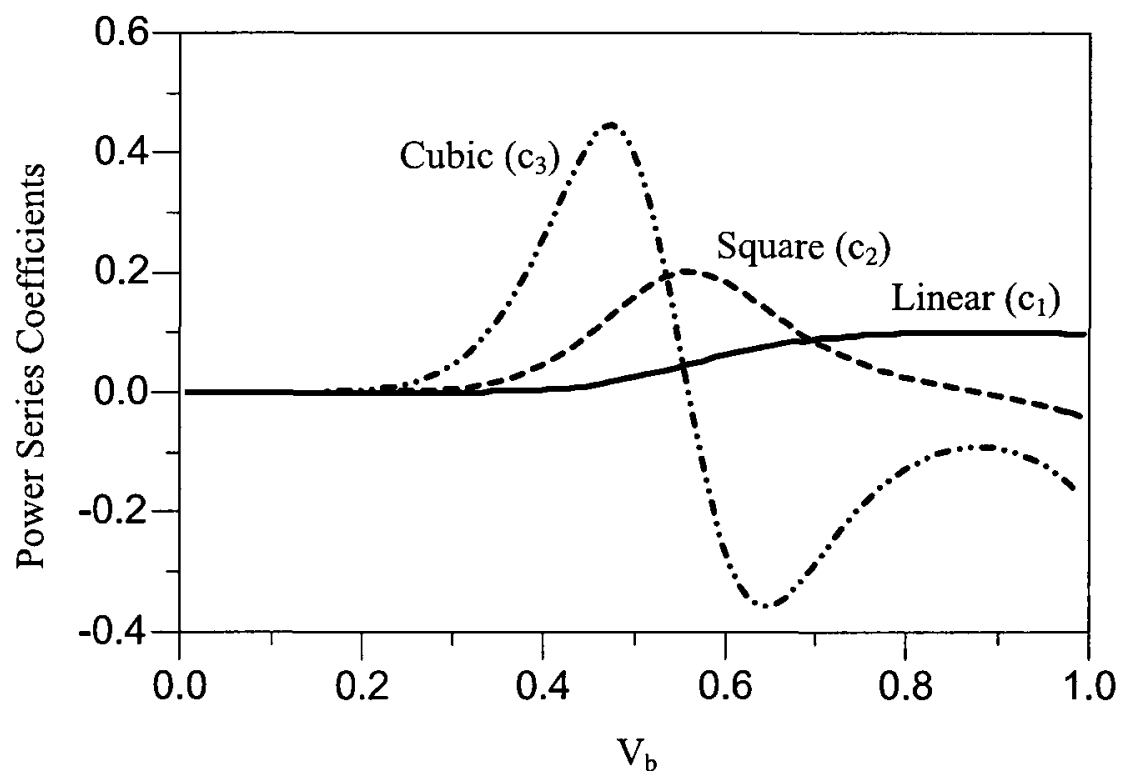

Figure 4.3: Coefficients of linear, square and cubic terms.

The linear term in (4.1.4) can be removed using two identical transistors driven by differential input, as shown in Figure 4.4 a. The total output current is

$$
\begin{aligned}
I_{1}+I_{2} & =c_{0}+c_{1} v_{i}+c_{2} v_{i}^{2}+c_{0}+c_{1}\left(-v_{i}\right)+c_{2}\left(-v_{i}\right)^{2} \\
& =2 c_{0}+2 c_{2} v_{i}^{2},
\end{aligned}
$$

which appears to be a perfect square-law relationship. However, it should be noted that Taylor series expansion is valid for the small signal operation. The input signal swing should be carefully arranged such that the circuit operates according to the Taylor series approximation.

The drawback of this two transistor circuit is the requirement of differential input, which is inconvenient for measurement. A single transistor was used as the squaring circuit in the prototype design and shown in Figure $4.4 \mathrm{~b}$. The linear term is retained based on following considerations: 1) single input, which is easy to drive; 2) because of the 
voltage headroom, it is hard to make the next stage linear and the third order distortion is unavoidable; therefore, the linear term is used to amplify the input, drive the next stage and utilize the next stage to produce the $X^{3}$ term; 3) producing $X^{3}$ term at the next stage reduces the bandwidth requirement of the first stage; 4) the linear term can serve in a linear measurement for a frequency sweeping; thus to produce the frequency responses of the tank circuits for observation purpose, which will be described in Chapter 6 .

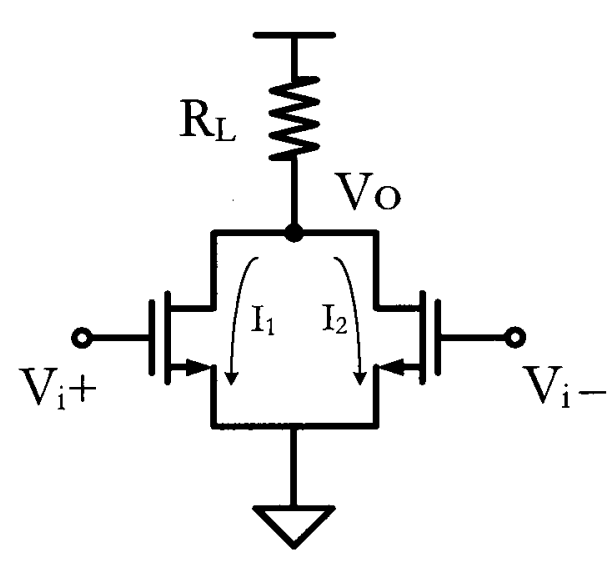

(a)

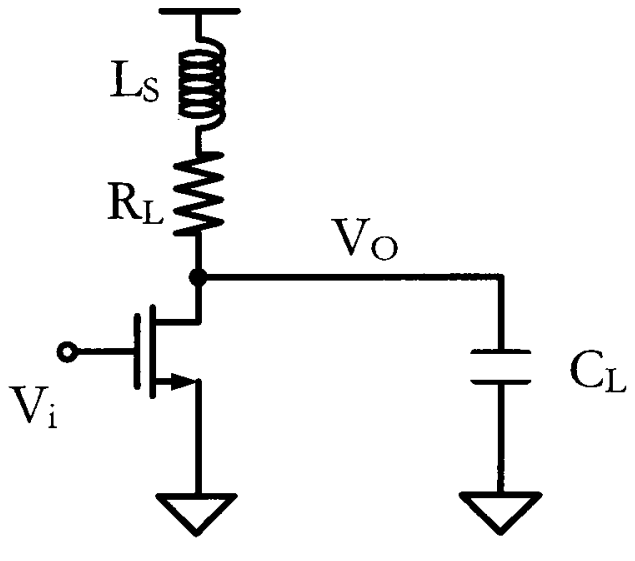

(b)

Figure 4.4: a. Two transistor squaring circuit. b. Common source amplifier.

$C_{L}$ in Figure 4.4 represents the load from next stage and also includes the parasitic drain-source capacitance of the transistor. $R_{L}$ is used to convert current to voltage and $L_{S}$ is used for inductive peaking to extend the output bandwidth. The total output impedance is

$$
Z_{O}=\frac{1}{\frac{1}{R_{L}+j \omega L_{S}}+j \omega C_{L}}
$$

For a maximally flat amplitude response,

$$
\left.\frac{\partial^{2}\left|Z_{O}\right|}{\partial \omega^{2}}\right|_{\omega=0}=0
$$

Solving (4.1.8), we have

$$
L_{S}=(\sqrt{2}-1) C_{L} R_{L}^{2} \approx 0.414 C_{L} R_{L}^{2}
$$


where $C_{L}$ is determined by the next stage and $R_{L}$ is determined by the dc gain. Similarly, for a maximally flat group delay response,

$$
\left.\frac{\partial^{3} \angle Z_{O}}{\partial \omega^{3}}\right|_{\omega=0}=0
$$

Solving (4.1.10), we have

$$
L_{S} \approx 0.322 C_{L} R_{L}^{2}
$$

Therefore, $L_{S}$ is chosen between $0.414 C_{L} R_{L}^{2}$ and $0.322 C_{L} R_{L}^{2}$ for a reasonable amplitude and group delay performance. If the bandwidth is not sufficient, we will have to either reduce dc gain (by reducing $R_{L}$ ) or scale down the transistor sizes of the next stage to reduce $C_{L}$.

\subsection{2 $\quad X^{3}$ Component and Tunable Gain Cell}

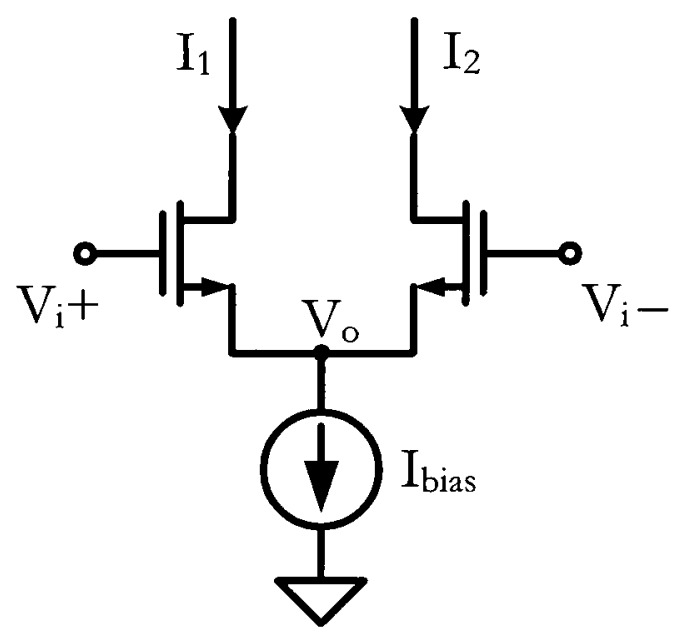

Figure 4.5: A differential pair.

Figure 4.5 shows a common source biased differential pair. We have

$$
\begin{aligned}
& I_{1}=\frac{K}{2}\left(V_{i+}-V_{o}-V t h\right)^{2} \\
& I_{2}=\frac{K}{2}\left(V_{i-}-V_{o}-V t h\right)^{2} .
\end{aligned}
$$

The differential output current and the differential input voltage are defined as

$$
\begin{aligned}
& I_{d}=I_{1}-I_{2} \\
& V_{d}=V_{i+}-V_{i-} .
\end{aligned}
$$


According to KCL,

$$
I_{1}+I_{2}=I_{b i a s}
$$

Using (4.1.12), (4.1.13) and (4.1.14), it can be obtained that

$$
I_{d}=\frac{1}{2} V_{d} \sqrt{4 I_{b i a s} K-K^{2} V_{d}^{2}}
$$

Rewrite (4.1.15) in Taylor series expansion and neglect terms higher than third order, we have

$$
I_{d}=\sqrt{I_{\text {bias }} K} V_{d}-\frac{1}{8} \sqrt{\frac{K^{3}}{I_{\text {bias }}}} V_{d}^{3} .
$$

From (4.1.16), we see the gain of the cubic term decreases with the increase of bias current. On the other hand, reducing bias current gradually turns off the transistor, thus decreasing the cubic term. Therefore, absolute value of the cubic term coefficient has a maximum when plotted versus bias current, as shown in Figure 4.6.

From this figure, it can be seen that tuning the bias current changes both the linear gain and the cubic gain, but does not change signs. In order to provide both positive and negative gain, the MOS version of Gilbert cell [61] is used to drive the LC tank circuit, as shown in Figure 4.7. Transistors M1, M2 and M3, M4 are two differential pairs crosscoupled. One pair is to provide positive gain and the other is to provide negative gain. The bottom differential pair by transistor M5 and M6 controls the bias current of the crosscoupled two pairs. By steering current from one differential pair to the other, positive or negative gain can be achieved.

Figure 4.8 illustrates how the bias current affects the third order distortion in the Gilbert cell. In the illustration, the overall cubic coefficient is the difference between the cubic coefficients of the two differential pairs. The tuning voltage $\left(V_{\text {tune }}\right)$ increases the bias current of one differential pair while reducing that of the other differential pair. The total bias current $\left(I_{\text {bias }}\right)$ determines how far apart the bias current of each pair with the maximum cubic term $\left(c_{3}\right)$ are. As demonstrated in figure $4.8(\mathrm{a})$, if $I_{b i a s}>2 I_{c 3 @ \max }\left(I_{c 3 @ \max }\right.$ is the bias current where $c_{3}$ is at peak), due to the curve shape of the cubic term versus tuning voltage, the overall cubic coefficient does not monotonically follow the tuning voltage. Reducing 


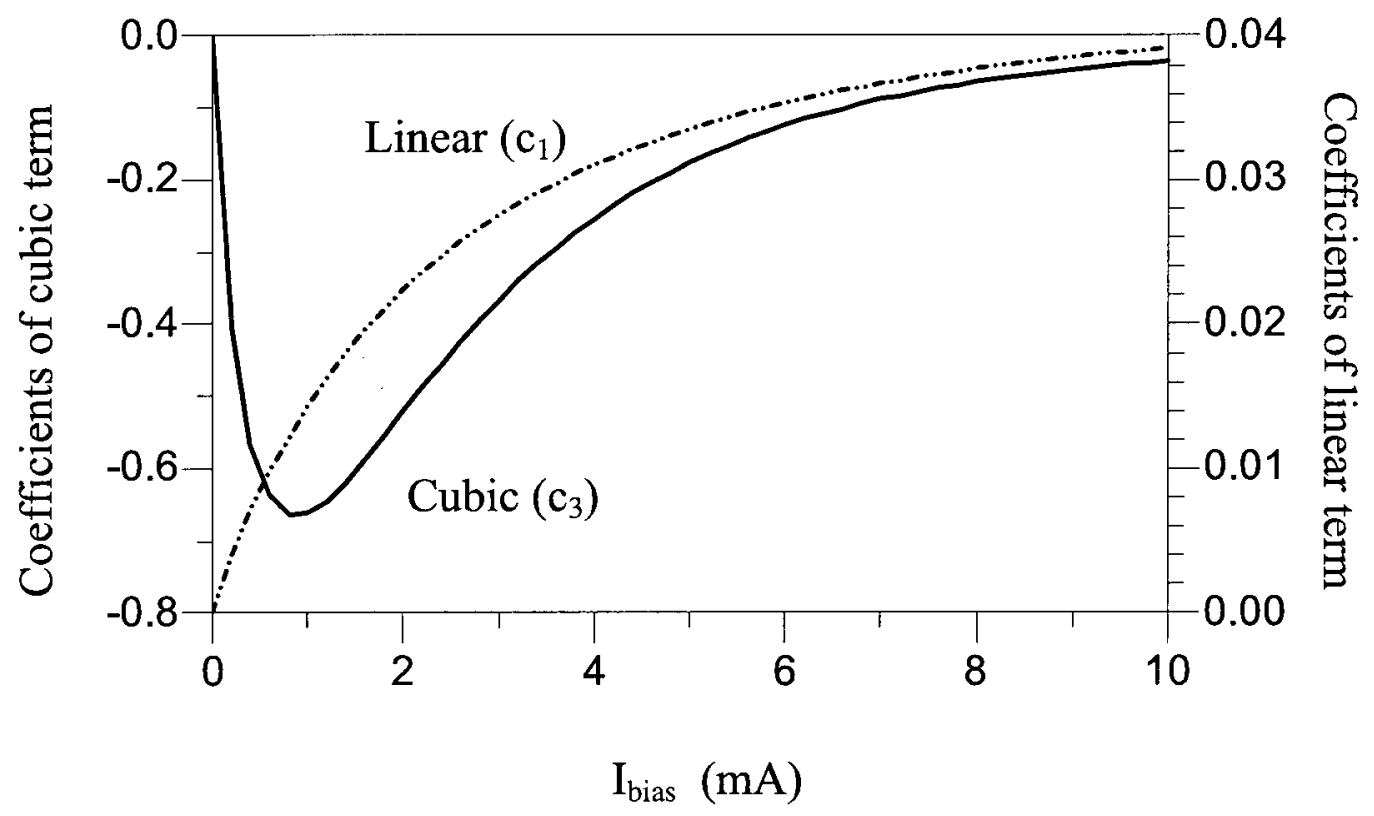

Figure 4.6: Linear and cubic coefficients of the differential pair (with the transistor size of $\mathrm{W}=40 \mu \mathrm{m}, \mathrm{L}=0.18 \mu \mathrm{m}$ and 4 fingers). 


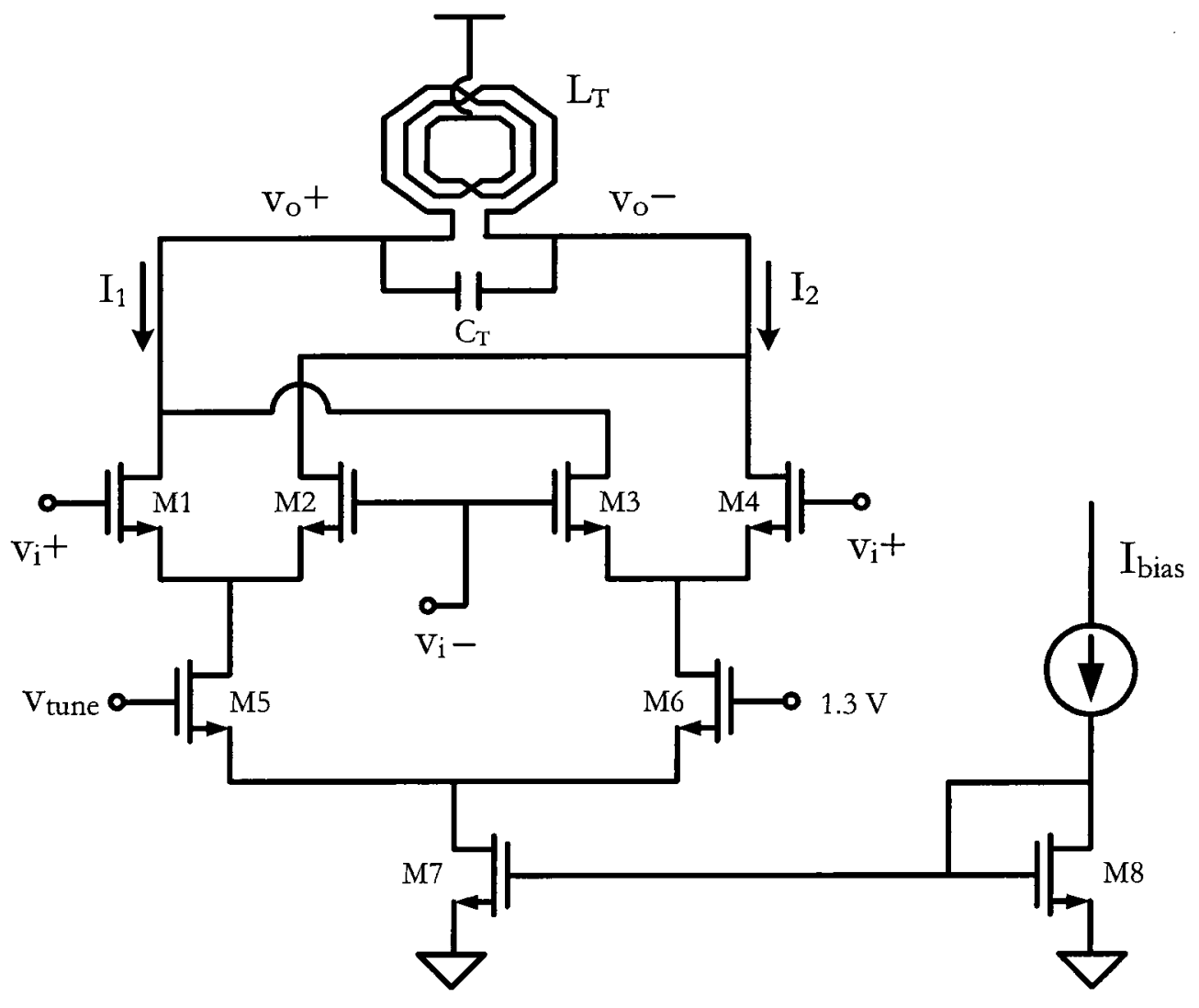

Figure 4.7: Tunable linear and cubic gain cell. 

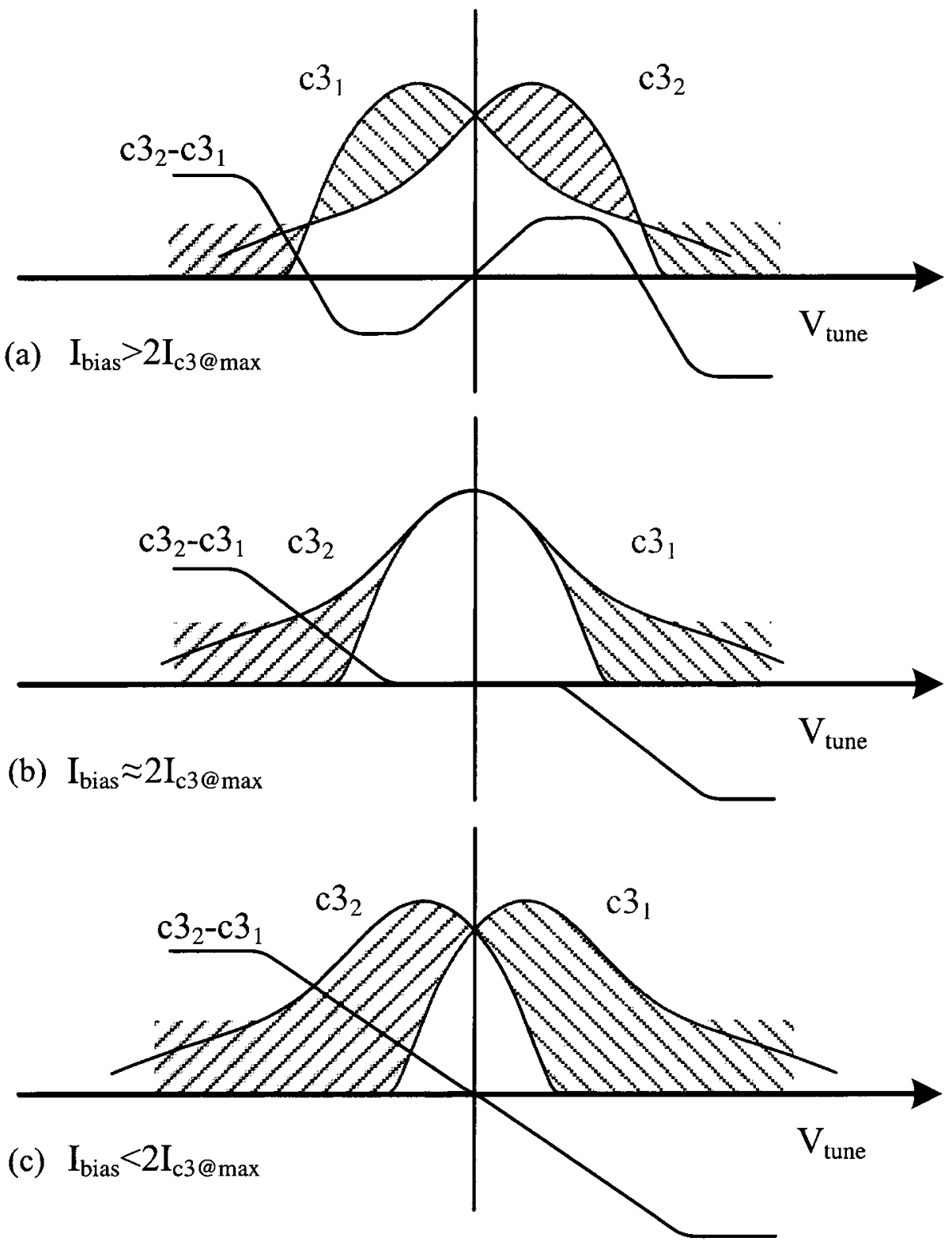

Figure 4.8: Third order distortion cancelation using Gilbert cell. 


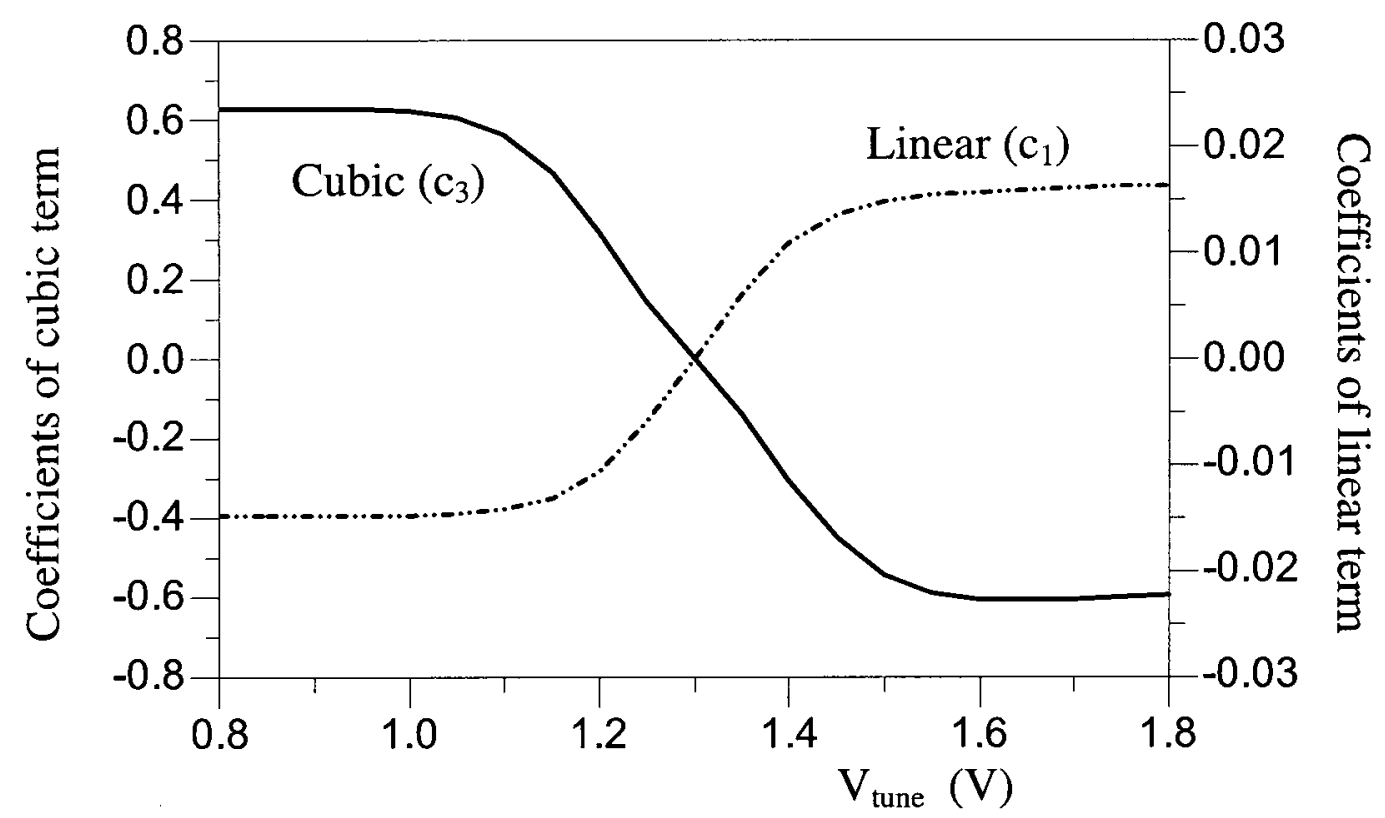

(a)

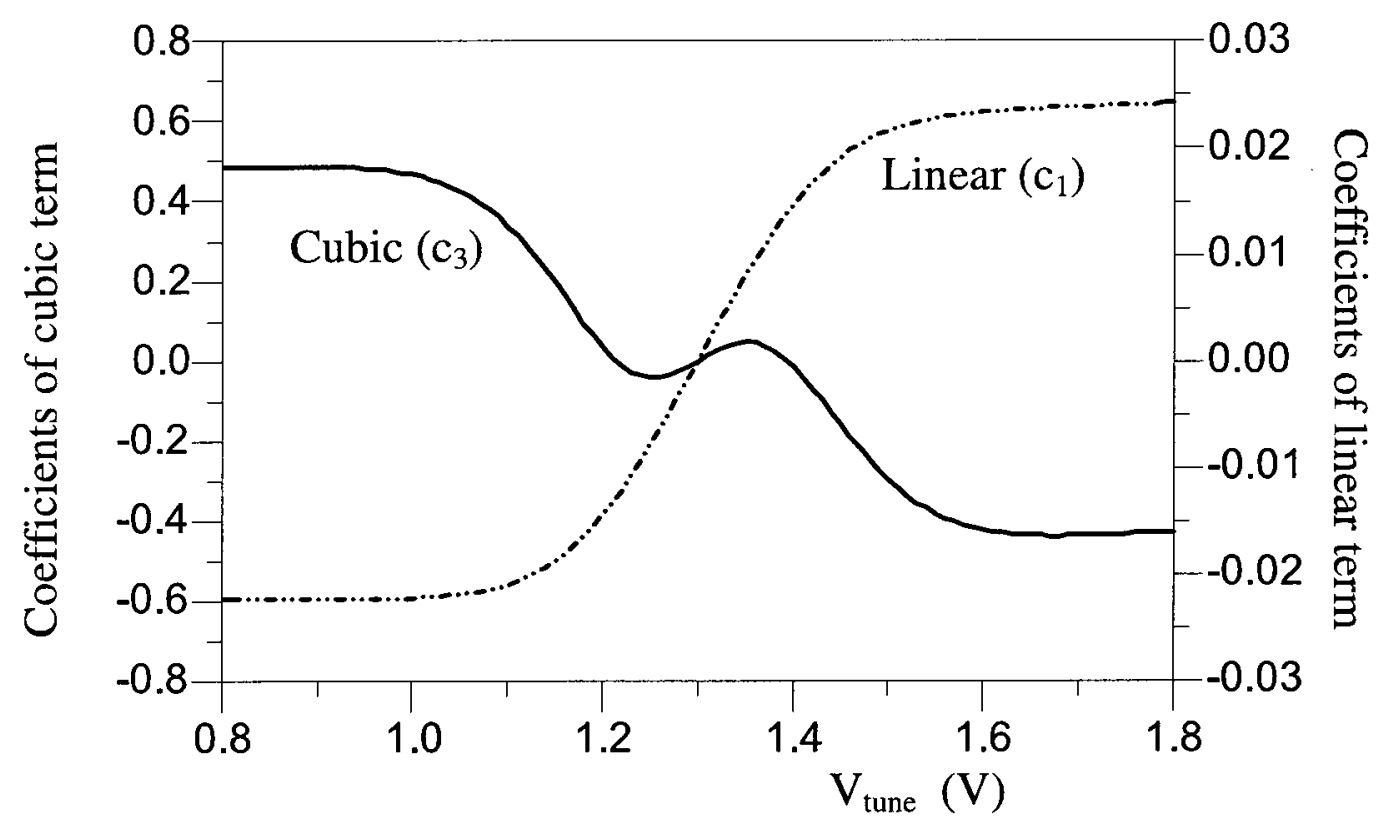

(b)

Figure 4.9: Linear and cubic coefficients of the tunable gain cell. (a) $I_{\text {bias }}=1 \mathrm{~mA}$. (b) $I_{\text {bias }}=2 \mathrm{~mA}$. 
the bias current so that the $c_{3}$ peak overlaps creates a flat region where the overall cubic term is zero as illustrated in figure 4.8 (b). A monotonic relationship between the cubic term and the tuning voltage can be achieved by reducing the bias current, as illustrated in figure 4.8 (c). Therefore, the total bias current $I_{\text {bias }}$ is set to $1 \mathrm{~mA}$ in the cubic circuit for a monotonic relationship and a small linear gain $\left(c_{1}\right)$. For linear circuit, the bias current is set to $2 \mathrm{~mA}$ to achieve a larger linear gain and small cubic gain. Figure 4.9 (a) and (b) shows the simulated results for $1 \mathrm{~mA}$ and $2 \mathrm{~mA}$ bias currents respectively.

The tunable gain cell described in this section is used as a tunable cubic term for the $X^{3}$ path, and provides tunable linear gain for the $X^{2}$ path. Cross-coupled differential pair described in [58] can be applied to remove the unwanted term in the output. However, it is not quite necessary and complicates the circuit design. For the unwanted $X^{3}$ signal created by the tunable gain cell in the $X^{2}$ path, because they are not in the same band as the $X^{2}$ signal, they will be mostly removed by the bandpass LC tank. The unwanted linear signal created by the tunable gain cell in the $X^{3}$ path, compared with the signal in the linear path, is small and can be simply neglected. Furthermore, the predistorter topology and the design algorithm described in Section 3.3 of Chapter 3 allow for the use of coarse $X^{2}$ and $X^{3}$ distortion generators.

The LC tank circuit is driven by the output current of the tunable gain cell to produce an output voltage. The inductors in the tank circuits are generally not required for high quality factor in the design, their layout area can be minimized using thin metal trace. Center tapped spiral configuration can be applied to further reduce the chip area.

The tunable gain should be digitally controlled due to the fact that analog value can not sustain by itself for a long time in the presence of leakage and noise. This digital control can be realized using a digital to analog converter (DAC). Since the accuracy is not a major concern of this DAC, a simple circuit will do the job. Figure 4.10 shows a tunable gain cell with digitally controlled gain. The DAC function is done by switching the current one way or the other. This circuit is still a Gilbert cell but in a different configuration where the bottom differential pair using $n$-type transistor is mapped to p-type by using current mirrors. This configuration reduces the number of stacked transistors between the 


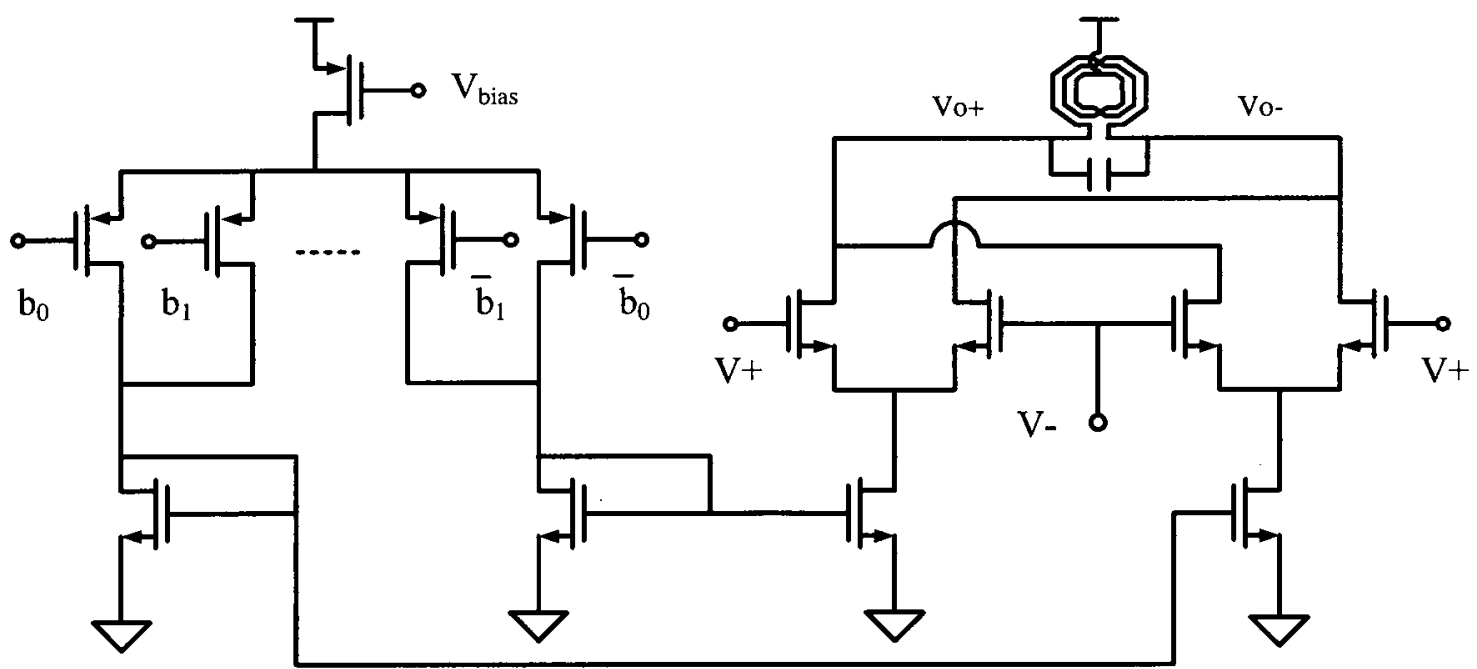

Figure 4.10: Circuit of a digitally tunable gain cell.

power supply and ground, therefore increase large signal operating range. It also separates the DAC function and the gain cell, which reduces the routing difficulty when doing layout.

\subsubsection{Laser Driver and Summing}

The laser drivers convert the drive voltages from the tunable gain cells to currents. The summing function is realized by connecting the laser driver outputs together, so the output current is the sum of the currents from each driver.

Figure 4.11 shows the circuits of a multi-tanh linearized differential pair and a simple differential pair. Figure 4.12 portrays the basic principle of multi-tanh linearization [62]. $g m_{1}$ and $g m_{2}$ are the transconductances of the two differential pairs in the multi-tanh configuration ( $c_{1}$ in their Tyler series expansion of V-I relationship). The offset voltage shifts the center of the two $g m s$ apart, so that the total $g m$ has a wider flat region. The derivative of $g m$ is $c_{2}$, which stays constant at near 0 for a wider range. Remember that the cubic term is the derivative of $c_{2}$, hence the cubic term becomes near zero for a certain range, as illustrated in Figure 4.13 (a).

Figure 4.13 (b) shows the linear and cubic coefficients of the simple differential pair configuration. Comparing Figure 4.13 (a) and (b), we can see that even though multi-tanh 


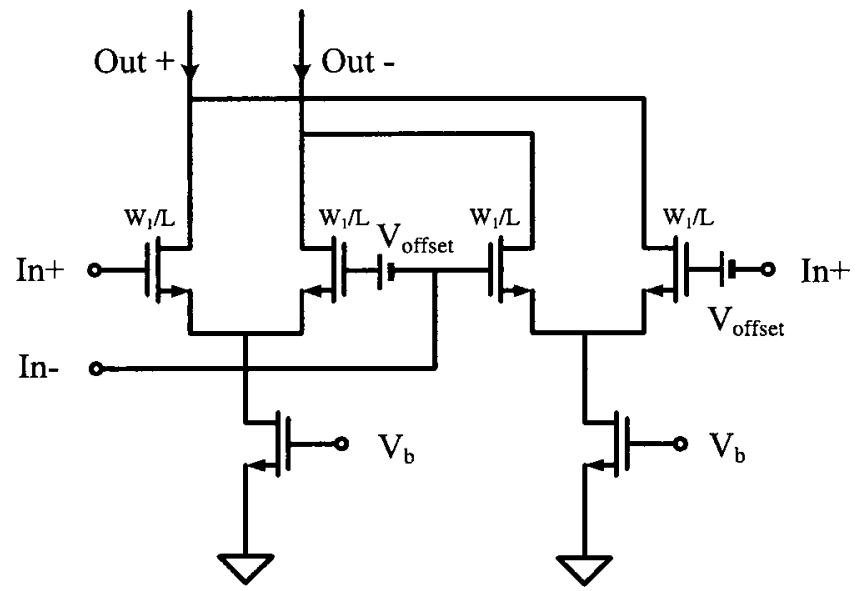

(a)

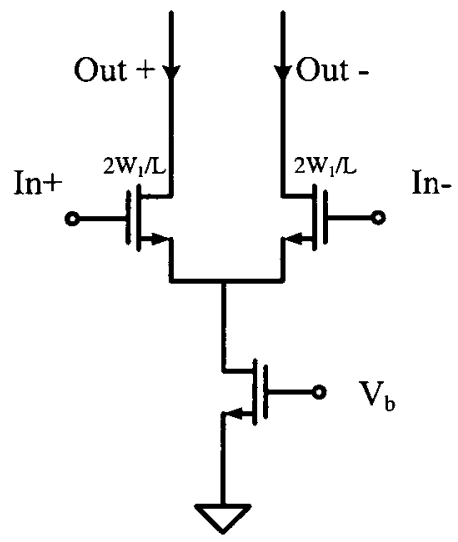

(b)

Figure 4.11: Laser driver (a) multi-tanh linearization (b) simple differential pair.

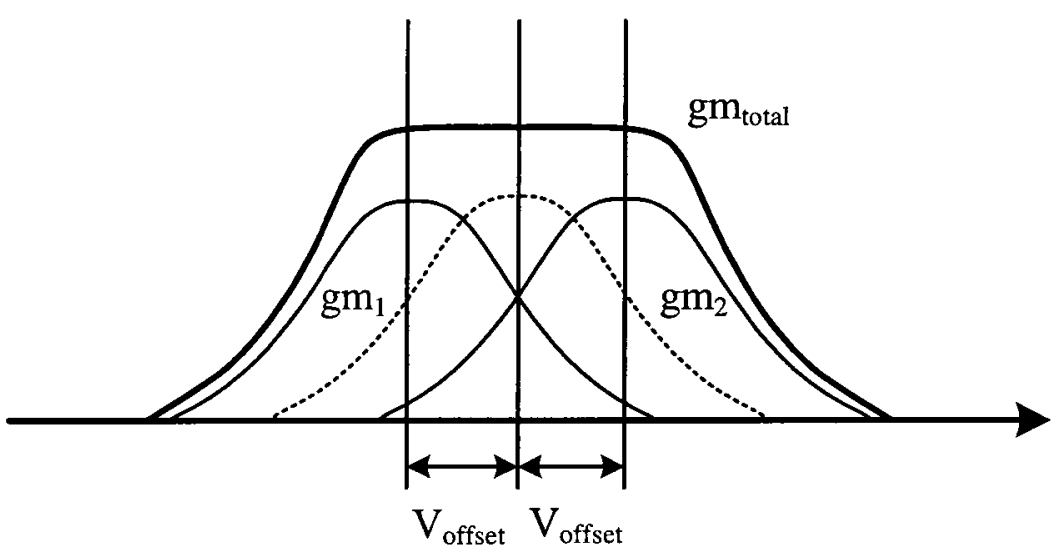

Figure 4.12: Multi-tanh linearization principle 
provides better linearity, but the linear gain reduced almost by half. The gain can increase by scaling up the transistor sizes. However, it will also increase the output parasitic capacitance, which reduces the operating bandwidth of the circuit. The linearity of the simple differential pair can be improved by increasing the bias current, as illustrated in Figure 4.14 (b). Increasing bias current also increases the gain for the simple differential pair configuration. The predistorter structure, even though derived from the laser inverse model, is a general structure which should be able to compensate up to third order distortion introduced by the laser driver. Also considering that the multi-tanh configuration needs an extra DC bias to provide the offset voltage, the simple differential pair configuration is chosen in the prototype design to save chip area and power consumption.

The outputs of laser drivers can be connected directly or in a distributed fashion as illustrated in Figure 4.15. The group delay introduced by the distributed network can be compensated by adjusting the frequency profile shaping filters. The price to pay is the use of many inductors. Again, to save the chip area, direct connection is chosen in the prototype design. 


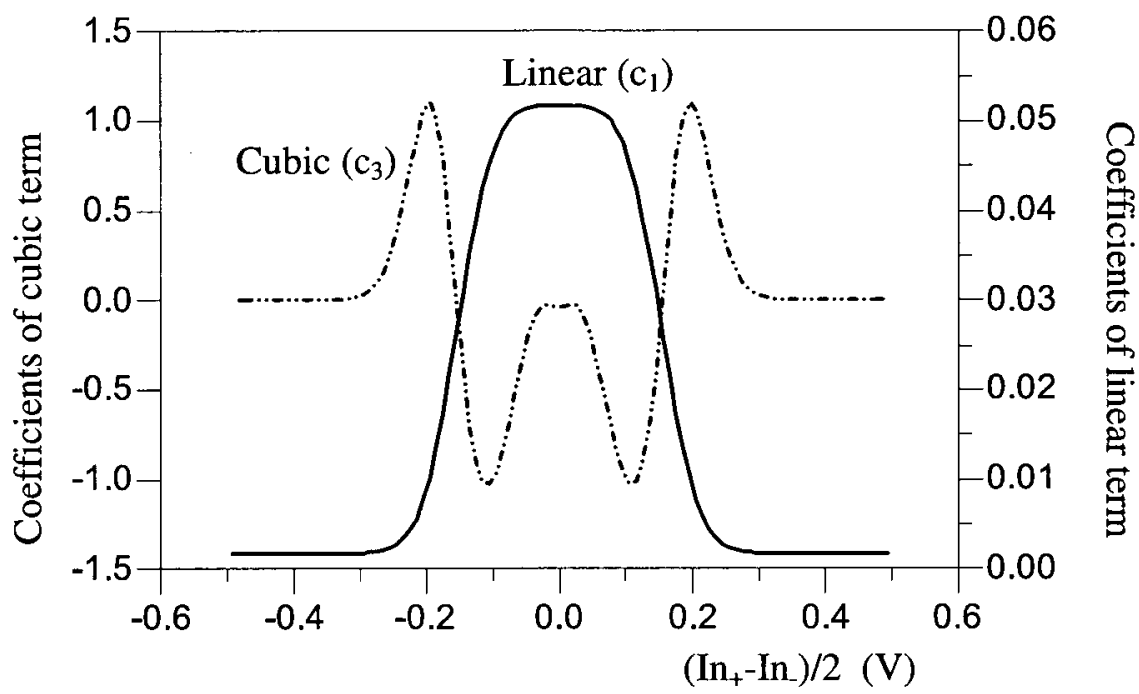

(a)

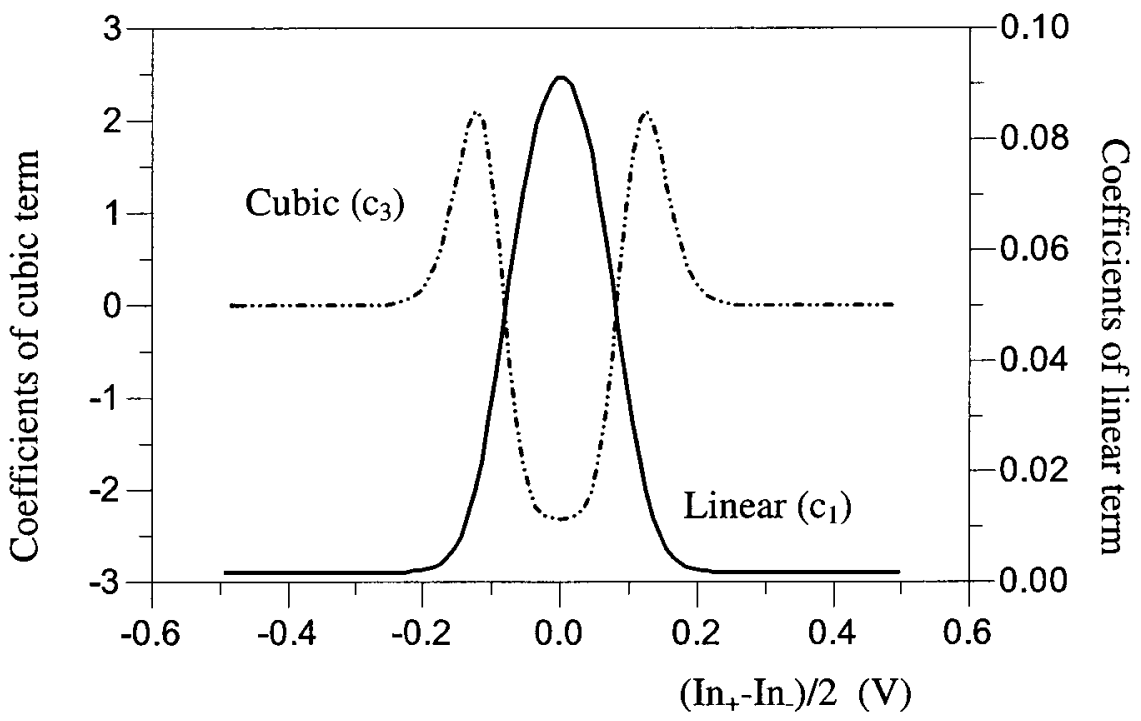

(b)

Figure 4.13: Linear and cubic coefficients of (a) multi-tanh linearized pair and (b) simple differential pair $(\mathrm{W} 1=200 \mu \mathrm{m}, \mathrm{L}=0.18 \mu \mathrm{m})$. 


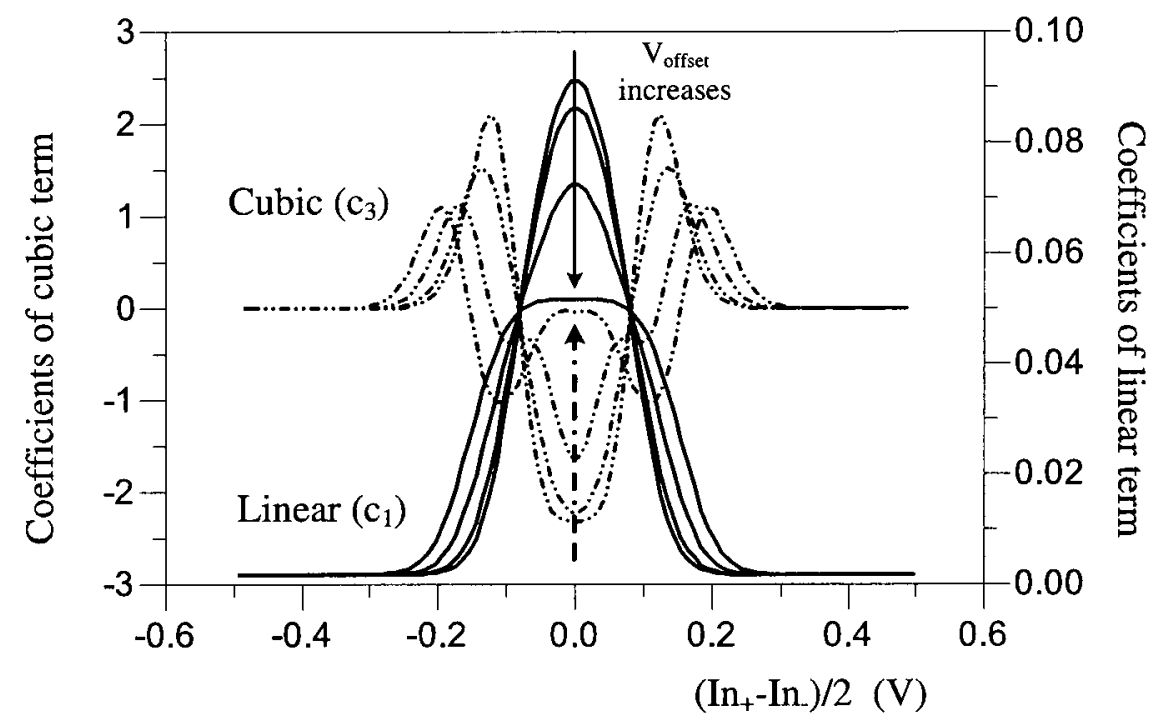

(a)

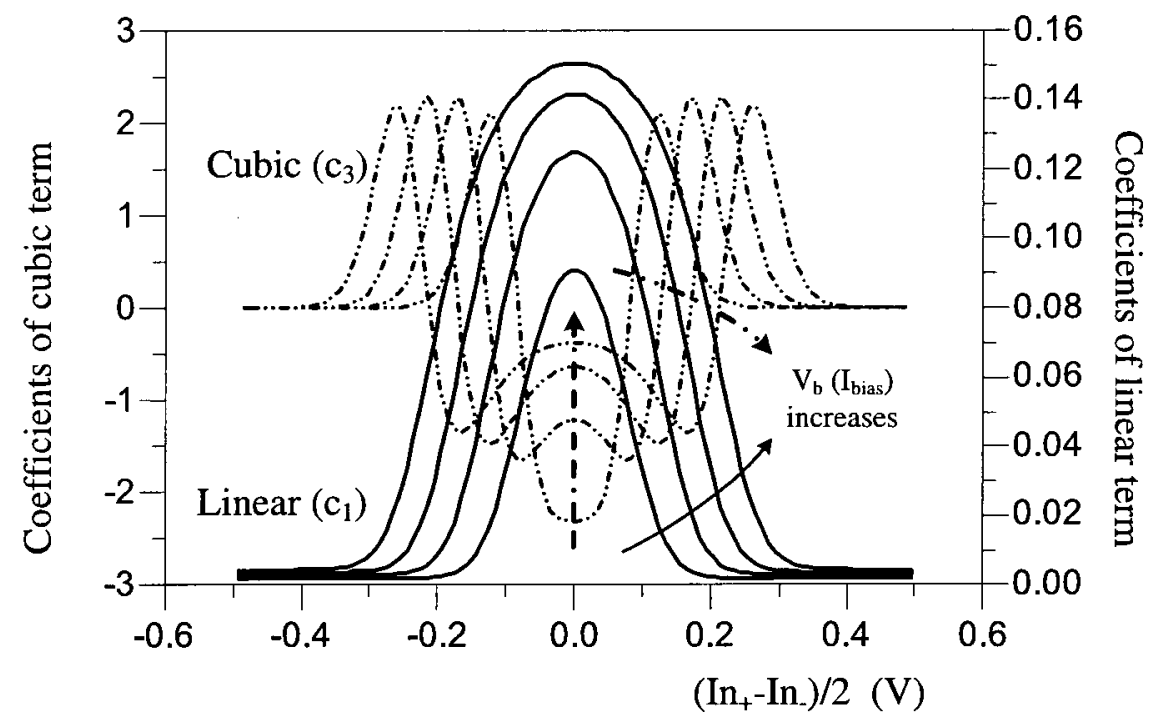

(b)

Figure 4.14: a) Effects of offset voltage on multi-tanh linearized pair and (b) effects of bias current on simple differential pair. 
(a)
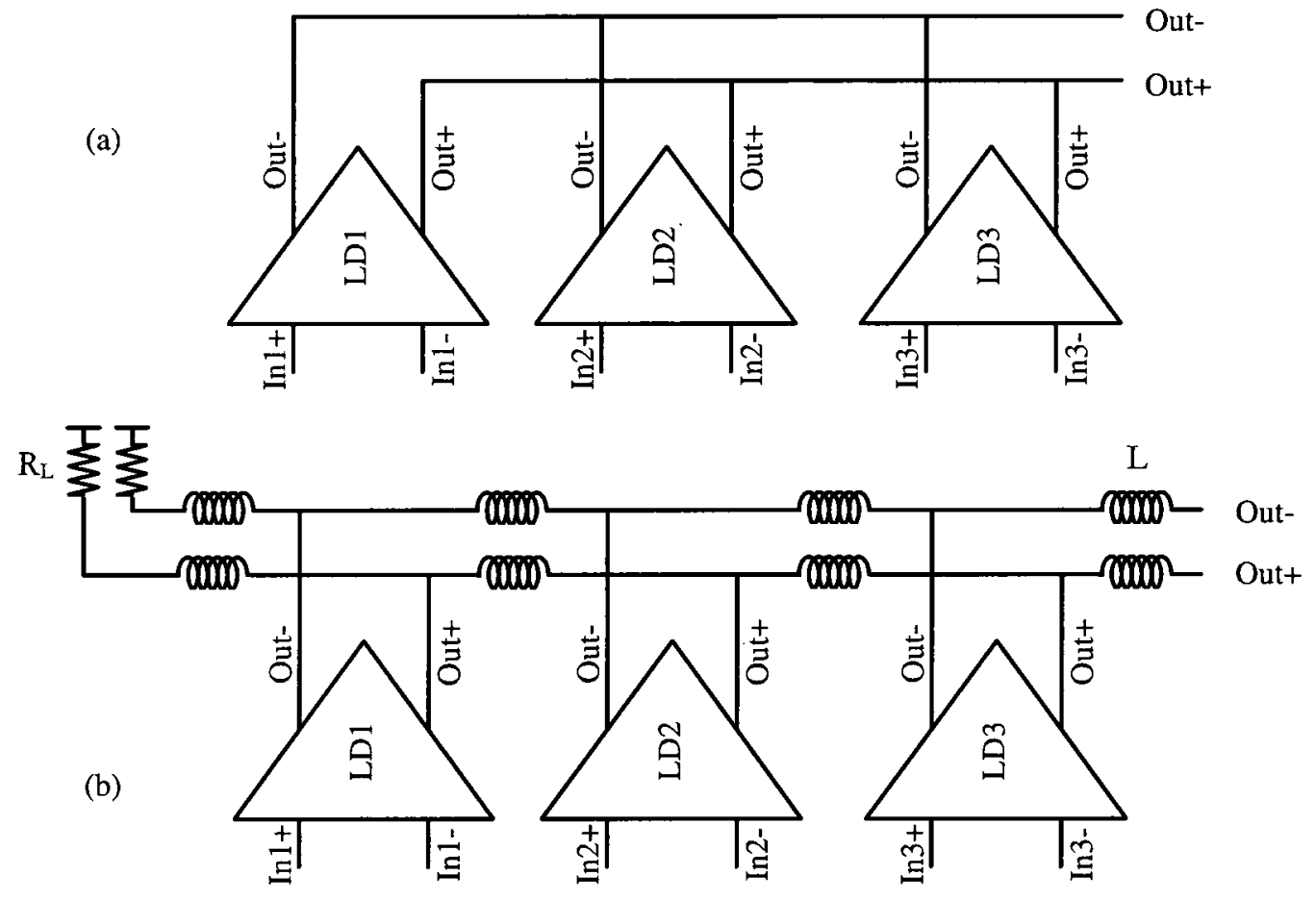

Figure 4.15: (a) direct (b) distributed current combining. 


\subsection{Design Example and Simulation Results}

Based on the proposed design approach, the design of a pre-distorter using standard 0.18 $\mu \mathrm{m}$ CMOS technology will be demonstrated in the section. Working frequency of $2 \mathrm{GHz}$ is chosen to demonstrate the capability for $\mathrm{GHz}$ range applications.

\subsubsection{Predistorter Model Parameters}

For the laser diode biased with $30 \mathrm{~mA}$ DC current, using the calculation procedure described in Chapter 3 Section 3.1 and the laser parameters listed in Table 2.1, the predistorter model can be obtained and the predistorter parameters are listed in Table 4.1.

\begin{tabular}{|c|c|c|c|}
\hline Shaping filters & dc & Coefficient of $s$ & Coefficient of $s^{2}$ \\
\hline \hline$G_{1}(s)$ & 1 & $5.03 \times 10^{-12}$ & $3.67 \times 10^{-22}$ \\
\hline$G_{2}(s)$ & $-7.86 \times 10^{-4}$ & $-6.65 \times 10^{-12}$ & $-8.21 \times 10^{-21}$ \\
\hline$G_{3}(s)$ & $9.16 \times 10^{-3}$ & $1.79 \times 10^{-10}$ & $2.48 \times 10^{-19}$ \\
\hline
\end{tabular}

Table 4.1: Predistorter model parameters

Figure 4.16 shows the simulation results of using the ideal predistorter model to drive the laser diode. It demonstrates the predistorter model works well with more than $10 \mathrm{~dB}$ optical intensity reduction for second order and third order distortions.

The linear signal path can be designed using passive filters to utilize the linearity of the passive components. A delay line is used in the linear path to simplify the design $[16,25,53,58,63,64]$. The filter response of the linear path becomes

$$
G_{1}^{\prime}(s)=e^{-s \cdot t_{d}}
$$

where $t_{d}$ is the time delay of the linear path. Using this linear path as the reference, the compensation for the square path shaping filter is found to be:

$$
G_{2}^{\prime}(s)=\frac{G_{2}(s)}{G_{1}\left(\frac{s}{2}\right)^{2}} e^{-s \cdot t_{d}}
$$

The output of the cubic path has two frequency bands: one is around third order harmonic components (HD3) and the other is around third order intermodulation components (IM3). 
1-tone test

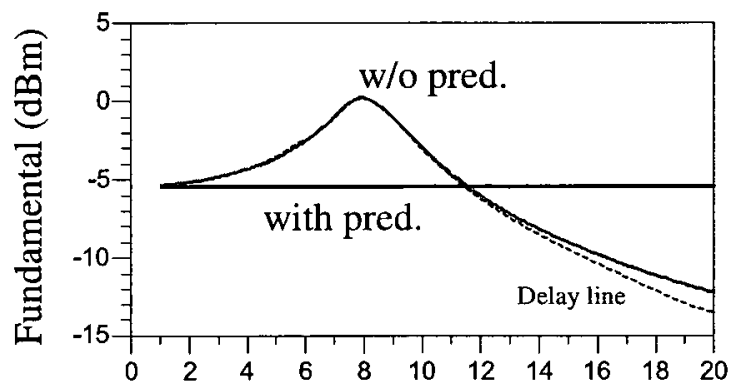

(a) Freq $(\mathrm{GHz})$

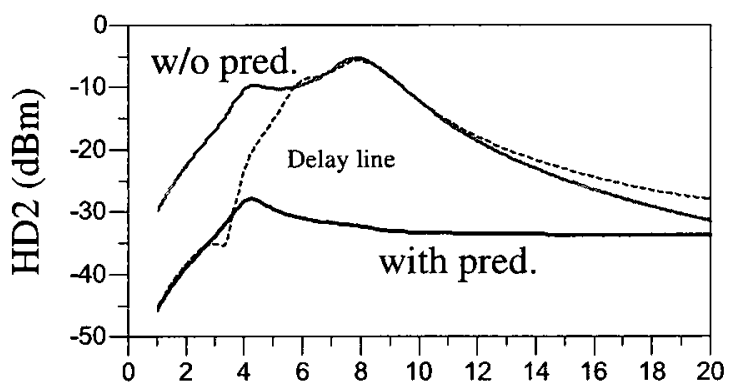

(c) Freq $(\mathrm{GHz})$

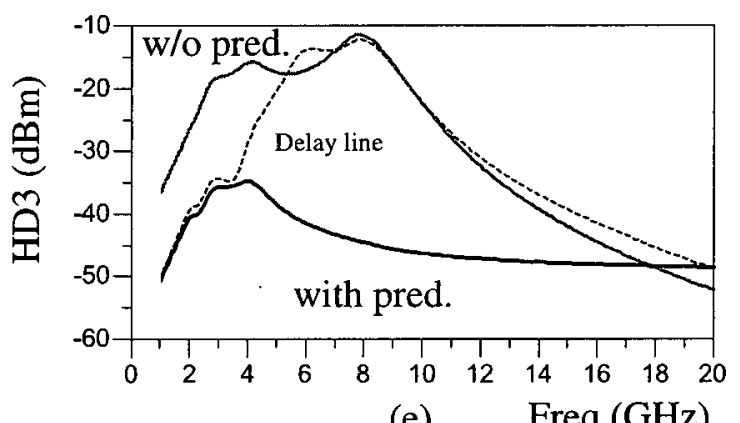

(e) Freq $(\mathrm{GHz})$ 2-tone test

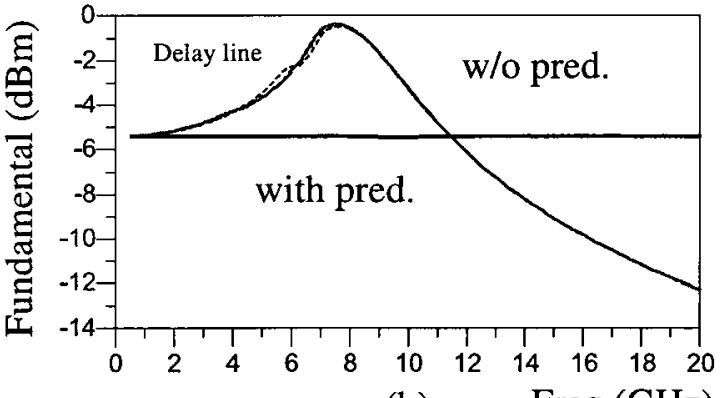

(b) Freq $(\mathrm{GHz})$

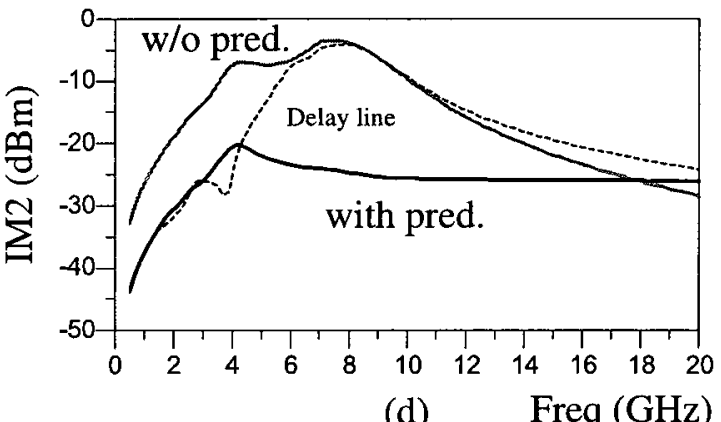

(d) Freq $(\mathrm{GHz})$

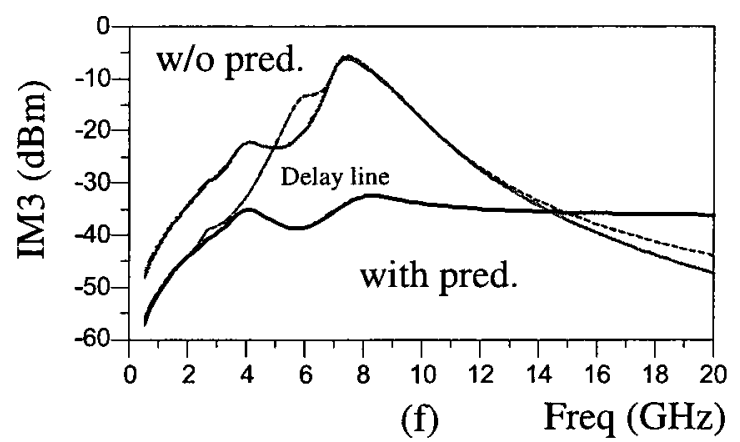

Figure 4.16: Ideal predistortion results by 1-tone simulation and 2-tone simulation; (a) (b) linear components optical intensity; (c) second order harmonic distortion optical intensity (d) second order intermodulation distortion at $f_{1}+f_{2}$; (e) third order harmonic distortion optical intensity (f) third order intermodulation distortion at $2 f_{1}-f_{2}$ (solid line) and $2 f_{2}-f_{1}$ (dotted line); $\left(I_{\text {bias }}=30 \mathrm{~mA}, I_{R F}=5 \mathrm{~mA}\right)$. The short dash lines are the results of using a delay line in the linear path. 
The compensation for the HD3 band filter response is found to be

$$
G_{3}^{\prime}(s)=\frac{G_{3}(s)}{G_{1}\left(\frac{s}{3}\right)^{3}} e^{-s \cdot t_{d}} .
$$

The compensation for the IM3 band filter response is found to be

$$
G_{3}^{\prime}(s)=\frac{G_{3}(s)}{G_{1}(s)^{2} G_{1}(-s)} e^{-s \cdot t_{d}} .
$$

The simulation results are shown in Figure 4.16 by short dash lines. It demonstrates that the predistorter works well from $\mathrm{DC}$ to $4 \mathrm{GHz}$ using a linear delay line.

\subsubsection{Frequency Band Allocation}

The targeted operating bandwidth is about $300 \mathrm{MHz}$, which will be enough for most of the wideband wireless standards, such as UMTS, WCDMA, Wireless LANs and WiMax. The predistorter output frequency range is $1.85-2.15 \mathrm{GHz}$ for the linear path, dc-150 MHz and 3.7-4.3 GHz for the quadratic path and 1.55-2.45 GHz and 5.55-6.45 GHz for the cubic path. The dc-150 MHz frequency band is neglected since the laser nonlinearity is quite low around DC and increases at high frequency, which represents as the high-pass feature of the profile shaping filter as shown in the Table 4.1. For the $X^{2}$ path, $3.7-4.3 \mathrm{GHz}$ is the frequency band of interest and HD2 component and IM2 product at $f_{1}+f_{2}$ fall in this band. For the $X^{3}$ path, IM3 product at $2 f_{1}-f_{2}$ or $2 f_{2}-f_{1}$ falls in $1.55-2.45 \mathrm{GHz}$ band. HD3 component and IM3 component at $2 f_{1}+f_{2}$ or $2 f_{2}+f_{1}$ fall in $5.55-6.45 \mathrm{GHz}$ band. Hence, there are two bands for the $X^{3}$ path. Considering the third order output of a cascaded two stages in (3.1.9),

$$
\begin{aligned}
Q_{3}\left(s_{1}, s_{2}, s_{3}\right)= & H_{3}\left(s_{1}, s_{2}, s_{3}\right) F_{1}\left(s_{1}+s_{2}+s_{3}\right)+H_{1}\left(s_{1}\right) H_{1}\left(s_{2}\right) H_{1}\left(s_{3}\right) F_{3}\left(s_{1}, s_{2}, s_{3}\right) \\
& +\frac{2}{3} H_{1}\left(s_{1}\right) H_{2}\left(s_{2}, s_{3}\right) F_{2}\left(s_{1}, s_{2}+s_{3}\right) \\
& +\frac{2}{3} H_{1}\left(s_{2}\right) H_{2}\left(s_{1}, s_{3}\right) F_{2}\left(s_{2}, s_{1}+s_{3}\right) \\
& +\frac{2}{3} H_{1}\left(s_{3}\right) H_{2}\left(s_{1}, s_{2}\right) F_{2}\left(s_{3}, s_{1}+s_{2}\right)
\end{aligned}
$$

where $H_{3}\left(s_{1}, s_{2}, s_{3}\right) F_{1}\left(s_{1}+s_{2}+s_{3}\right)$ is the third order term contributed by the predistorter third order output $\left(H_{3}\left(s_{1}, s_{2}, s_{3}\right)\right)$. The predistorter third order output simply passes 
through a linear filter $\left(F_{\mathbf{1}}(s)\right)$ to get to the laser output. The $5.55-6.45 \mathrm{GHz}$ band of the predistorter output goes directly to $5.55-6.45 \mathrm{GHz}$ band at the laser output. Since there is no frequency conversion for linear filters, signals in $5.55-6.45 \mathrm{GHz}$ band will not affect signals in $1.55-2.45 \mathrm{GHz}$ band. Thus, the $5.55-6.45 \mathrm{GHz}$ band in the $X^{3}$ path is neglected as the distortion in this band can be easily removed by a lowpass filter after the laser output. Therefore, the predistorter's frequency profile shaping filters will be approximated in the range of $3.7-4.3 \mathrm{GHz}$ for $X^{2}$ path and in the range of $1.55-2.45 \mathrm{GHz}$ for $X^{3}$ path using the proposed multiple tank technique.

\subsubsection{Distortion Generator Calculation}

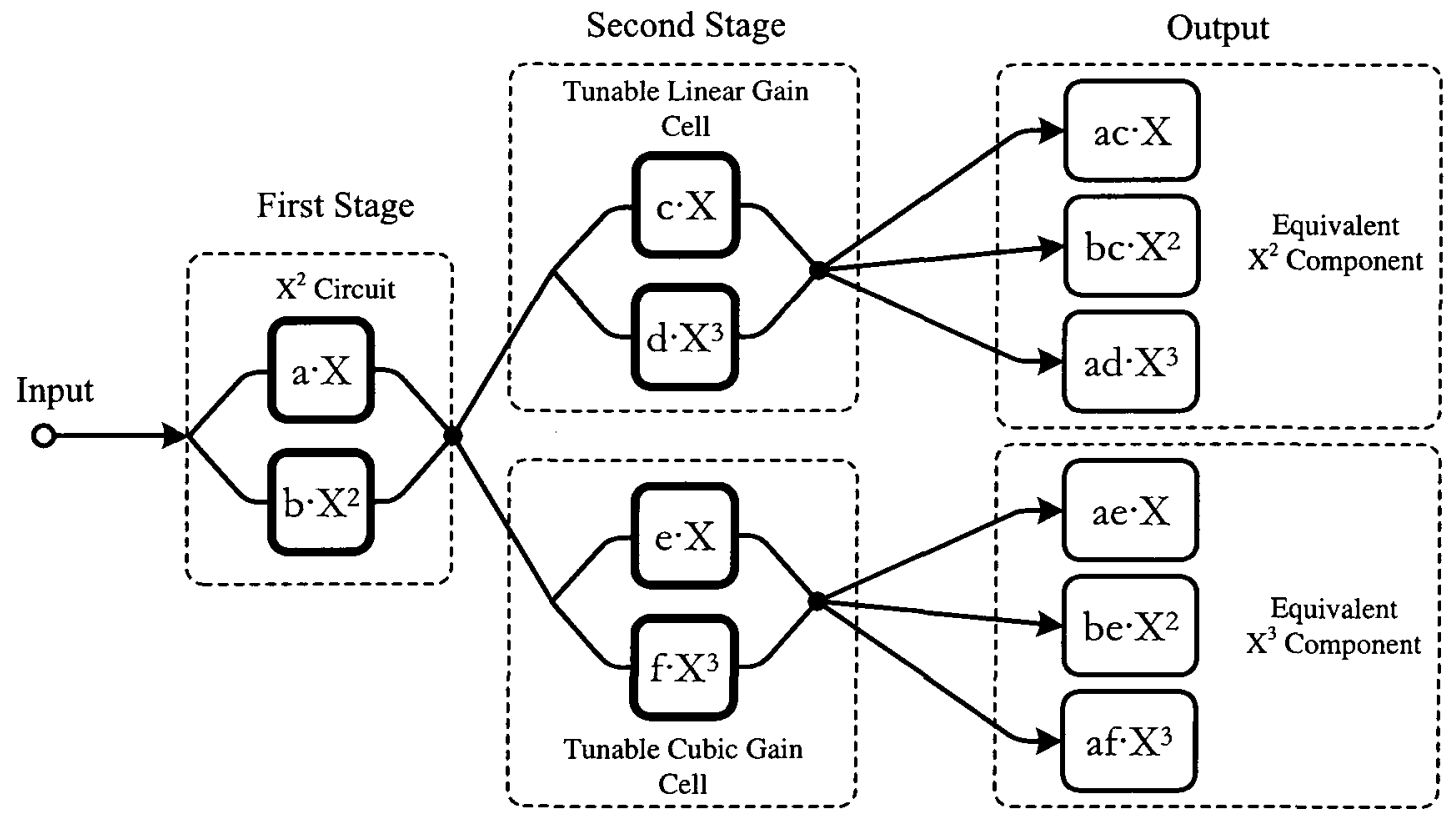

Figure 4.17: Obtaining the coefficients of distortion generators.

The circuit building blocks - $X^{2}$ circuit and the Gilbert cell described in previous section form the first stage and second stage of the predistorter. They function as $X^{2}$ and $X^{3}$ distortion generators and provide tunability on square and cubic coefficients. The output currents of the second stages can be represented as tunable distortion generators. Figure 
4.17 demonstrates the calculation of obtaining the equivalent $X^{2}$ and $X^{3}$ components, where

$$
\begin{array}{ll}
a=0.045 R_{L} & b=0.2 R_{L} \\
c=0.15\left(V_{\text {tune }}-1.3\right) & d=0.67\left(V_{\text {tune }}-1.3\right) \\
e=0.11\left(V_{\text {tune }}-1.3\right) & f=-3.23\left(V_{\text {tune }}-1.3\right)
\end{array}
$$

based on the simulation results of each circuit block in section 3. Therefore, neglecting higher order terms, the $X^{2}\left(D_{2}\right)$ and $X^{3}\left(D_{3}\right)$ components can be formulated as

$$
\begin{aligned}
& D_{2}=R_{L}\left(6.75 \times 10^{-3} x+3.0 \times 10^{-2} x^{2}+3.02 \times 10^{-2} x^{3}\right) \\
& D_{3}=R_{L}\left(4.95 \times 10^{-3} x+2.2 \times 10^{-2} x^{2}-1.45 \times 10^{-1} x^{3}\right)
\end{aligned}
$$

and the tunable gain can be formulated as

$$
a_{i}=\left(V_{\text {tune }_{i}}-1.3\right)
$$

$D_{2}$ has not only the square term, but also a small cubic term. The tank circuits connected to $D_{2}$ will be tuned to $3.7-4.3 \mathrm{GHz}$ band. The signals generated by the cubic term will be mostly removed by the tank circuit. Similarly, $D_{3}$ has not only the cubic term, but also the square term. The tank circuits connected to $D_{3}$ will be tuned to $1.55-2.45 \mathrm{GHz}$ band. The signals generated by the square term will be mostly removed.

Figure 4.18 shows the $\mathrm{AC}$ response of the $X^{2}$ component. It can be seen that the group delay variation in the $2-4 \mathrm{GHz}$ range is less than 2 ps. Compared with the period of a $2 \mathrm{GHz}$ signal, $0.5 \mathrm{~ns}$, the group delay variation is four hundredth of this signal period. Thus, the linear and square signals arrive at the second stage at approximately the same time. Therefore, the distortion generator formulation of (4.2.7), which is based on DC simulation, will be valid for a wide frequency range.

\subsubsection{Laser Driver Modeling}

Figure 4.19 shows the simulated $\mathrm{AC}$ response of the laser driver circuit. In the simulation, the laser diode is represented as a $50 \Omega$ load to match the impedance of the measurement equipment. The outputs of eight laser drivers will be connected together to the $50 \Omega$ load. The total output impedance has been included in the simulation of a single laser 


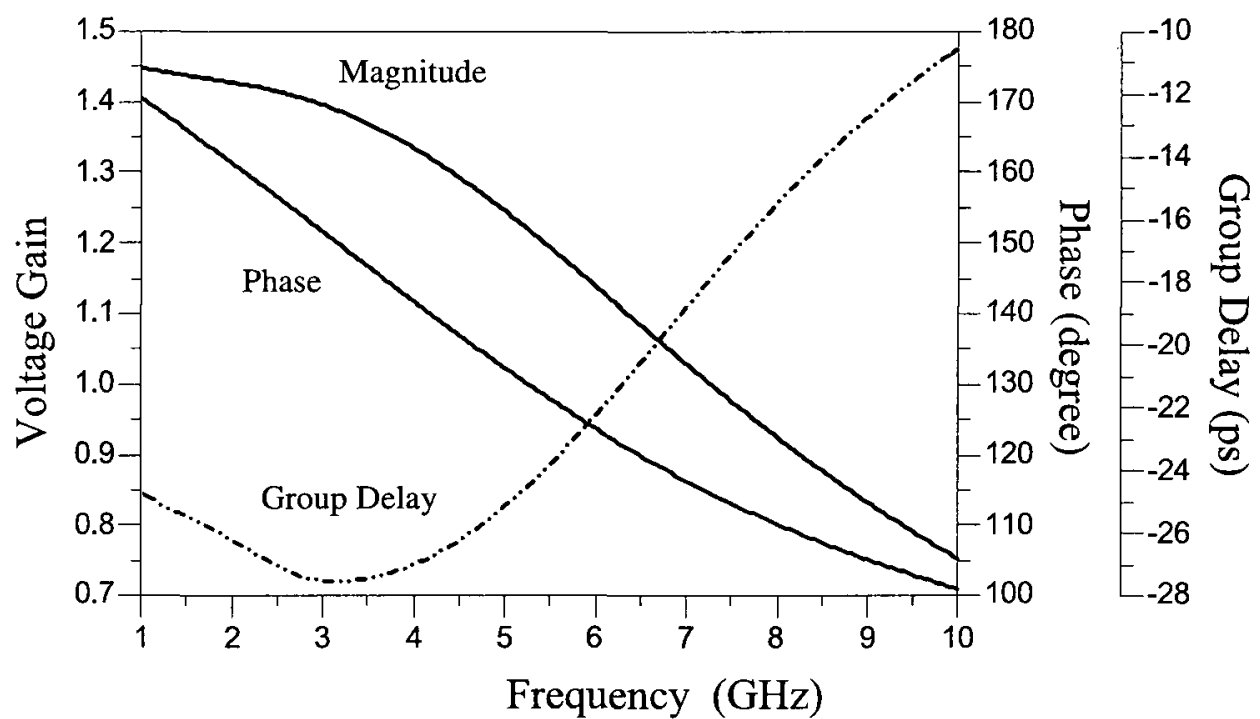

Figure 4.18: AC response of the $X^{2}$ circuit.

driver. For purpose of optimizing the tank circuits, the laser driver circuit is modeled by polynomial-fitting in the form of

$$
T_{L D}(s)=\frac{1}{\sum_{n=0}^{N} b_{n} s^{n}}
$$

to approximate the simulated frequency response. In Figure 4.19, the solid lines are the magnitude, phase and group delay of the simulated transconductance of the laser driver. The dotted line is the polynomial-fit results, where 10 terms are used in the fitting polynomial in order to reduce the group delay error to $2 \mathrm{ps}$ in $2-4 \mathrm{GHz}$ range. Table 4.2 lists the denominator polynomial coefficients obtained by curve-fitting using least-square method [65]. 


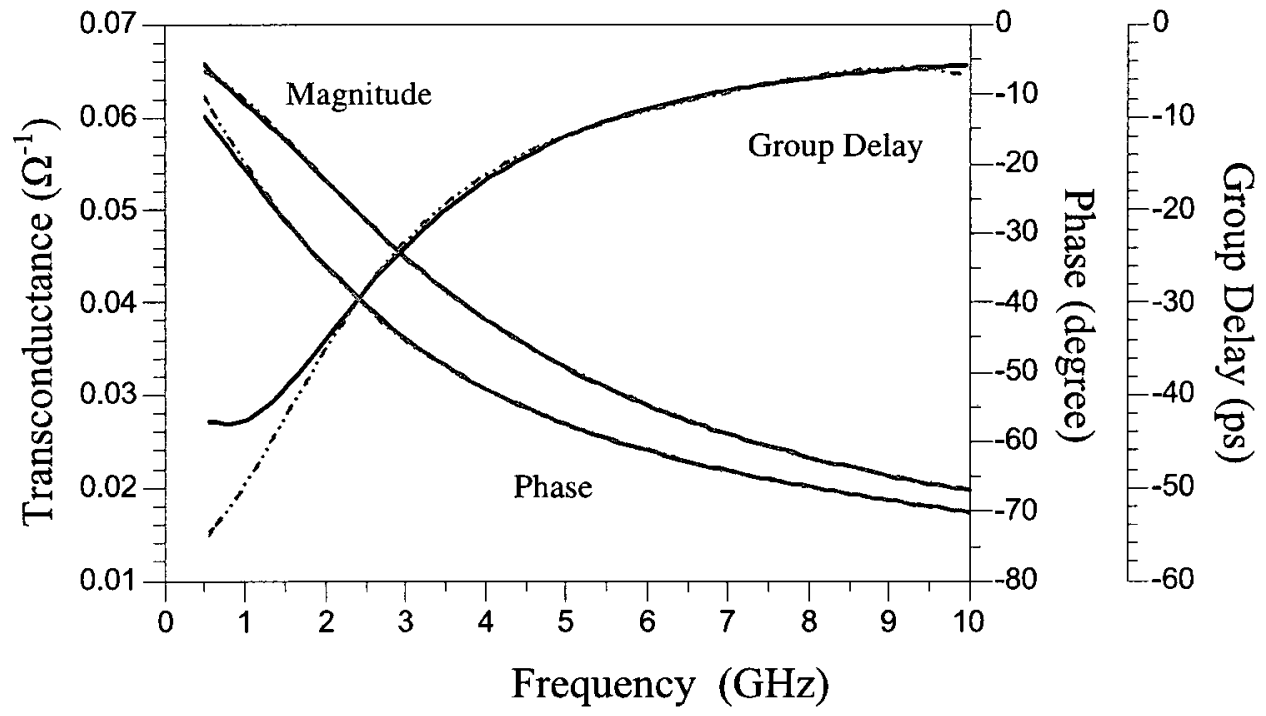

Figure 4.19: AC response of the laser driver circuit(simulation results: solid line, polynomial fit: dotted line.

\begin{tabular}{|c|c|c|c|c|}
\hline$b_{0}$ & $b_{2}$ & $b_{4}$ & $b_{6}$ & $b_{8}$ \\
\hline \hline-15.11 & $1.83 \times 10^{-21}$ & $8.03 \times 10^{-43}$ & $2.09 \times 10^{-64}$ & $2.26 \times 10^{-86}$ \\
\hline \hline$b_{1}$ & $b_{3}$ & $b_{5}$ & $b_{7}$ & $b_{9}$ \\
\hline \hline$-8.73 \times 10^{-10}$ & $-1.02 \times 10^{-31}$ & $-5.72 \times 10^{-53}$ & $-1.65 \times 10^{-74}$ & $-1.72 \times 10^{-96}$ \\
\hline
\end{tabular}

Table 4.2: Laser driver frequency response polynomial fit parameters. 


\subsubsection{Tank Circuits Optimization}

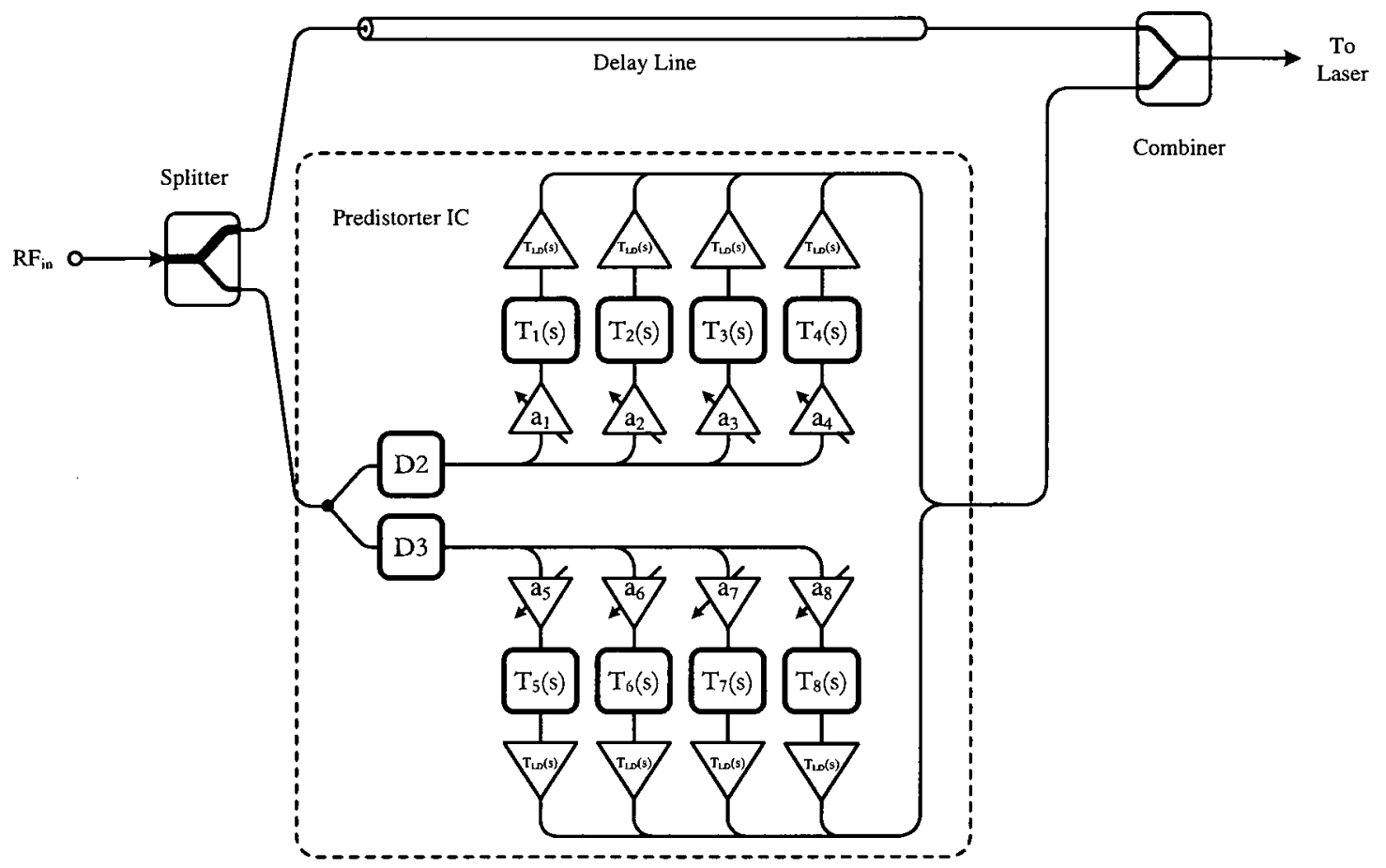

Figure 4.20: Block diagram of the predistortion system. The multitank predistorter IC is within the dash-line.

Figure 4.20 is the detailed block diagram of the predistortion system and the predistorter IC. Through the procedure described in the previous sections, $D_{2}, D_{3}$ and $T_{L D}(s)$ have been determined. The tuning coefficients $a_{i}$ can be obtained by solving the equations defined in (3.3.12) in Section 3.3 of Chapter 3. Therefore, different tank circuit bandwidth $\beta$ and center frequency spacing $d \omega$ can be exercised to minimized the mismatch between the profiles shaping filters and their multi-tank approximation. Including the frequency response of the laser driver stage, transfer functions of the tank circuits are modified as

$$
T_{m}(s)_{i}=T(s)_{i} \cdot T_{L D}(s)
$$

Heuristically, the time delay $t_{d}$ of the delay line should roughly match to that of the tank circuits in order to utilize the group delay of the tank circuit and to maximize the 
tunability. The group delay around the center frequency is related to the $3-\mathrm{dB}$ bandwidth $\beta$ of a tank circuit by

$$
t_{t a n k}=\frac{\partial \phi}{\partial \omega} \approx-\frac{2}{\beta}
$$

Therefore, the time delay of the linear path delay line should be

$$
t_{d}=t_{t a n k}+t_{L D}
$$

where $t_{L D}$ is the delay of the laser driver circuit. For a $300 \mathrm{MHz}$ operating bandwidth, the total delay obtained is about $1.1 \mathrm{~ns}$. However, this $1.1 \mathrm{~ns}$ only gives a starting point, different values of $t_{d}$ should be exercised to find the optimum time delay.

Using the calculation method described in Section 3.3 of Chapter 3 , the weighting coefficients $a_{i}$ can be obtained for each tank circuit. Therefore, different tank circuit parameters can be exercised to minimize the mismatch induced distortion using the procedure described in Section 3.4 of Chapter 3. The mismatch induced second order harmonic distortion is calculated by

$$
H D_{2}=\frac{(5 m A)^{2}}{2}\left(\frac{\int_{3.7 G H z}^{4.3 G H z}\left|\frac{\sum a_{i} T_{i}(s)-L(s)}{G_{1}(s)}\right|^{2} d f}{4.3 G H z-3.7 G H z}\right)^{1 / 2}=f_{1}(\beta, d \omega) .
$$

Similarly, the mismatch induced third order intermodulation distortion can be calculated by

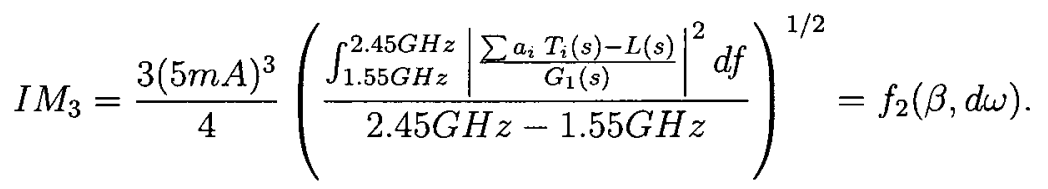

However, it is found that four tank circuits are not enough to cover this $900 \mathrm{MHz}$ IM3 band for a good distortion reduction. We reduce the integration range to $1.7-2.3 \mathrm{GHz}$ instead of using more tank circuits in the prototype design. $f_{1}(\beta, d \omega)$ and $f_{2}(\beta, d \omega)$ are both functions of tank circuit parameters $(\beta, d \omega)$. In this design example, four tank circuits are used for each path (square path and cubic path). The linear path time delay has been exercised from $0.5 \mathrm{~ns}$ to $1.5 \mathrm{~ns}$ with $0.05 \mathrm{~ns}$ step. Contour plots are used to search the optimum tank parameters for each time delay value, which are attached in Appendix B. The linear path 
time delay of $1 \mathrm{~ns}$ is chosen based on the contour plot results, as it produces acceptable mismatch induced distortions. The contour plot for $1 \mathrm{~ns}$ time delay is reprinted in Figure 4.21 .

As demonstrated in Figure 4.16 and Figure 4.22, the residual distortion is about -30 $\mathrm{dBm}$ for the second order harmonic distortion and about $-44 \mathrm{dBm}$ for the third order intermodulation distortion when using an ideal predistorter. In order to keep the performance degradation less than $3 \mathrm{~dB}$ in distortion reduction, the mismatch induced distortion should be less than the residual distortion. This gives the range for choosing the tank circuit parameters, roughly with $\beta>250 \mathrm{MHz}$ and $340>d \omega>160 \mathrm{MHz}$. Considering

$$
\beta=\frac{1}{R C}=\frac{\omega_{0}}{Q}
$$

(where $\omega_{0}$ is the center frequency and $Q$ is the quality factor of the tank circuit), a large bandwidth means a relatively low tank impedance and reducing the bandwidth needs to improve the quality factor of the on-chip inductor. The trade-off is between the necessary voltage gain produced through the tank circuit impedance and the chip area used used for high quality inductor.

In the design example, $2.4 \mathrm{nH}$ inductance is chosen for the IM3 band of the cubic path and $0.65 \mathrm{nH}$ is chosen for the HD2 band of the square path. The bandwidth $\beta$ of $600 \mathrm{MHz}$ and the frequency spacing $d \omega$ of $240 \mathrm{MHz}$ are chosen for the eight tank circuits in the design. Figure 4.22 is the simulation results using the circuits behavioral models defined in Section 4 of this chapter and ideal RLC tank circuits, where $d B c$ is defined as

$$
20 \log \left(\frac{\text { Optical Intensity of Distortion Component }}{\text { Optical Intensity of Fundamental Component }}\right) \text {. }
$$

The simulation results demonstrate a reduction of $18 \mathrm{~dB}$ for IM3 distortion using multitank approximation, whose performance is close to that of an ideal predistorter with about $1.5 \mathrm{~dB}$ difference. The reduction for HD2 distortion is $18 \mathrm{~dB}$ and for IM2 distortion is 20 $\mathrm{dB}$, which are about $3 \mathrm{~dB}$ more than these of an ideal predistorter. 


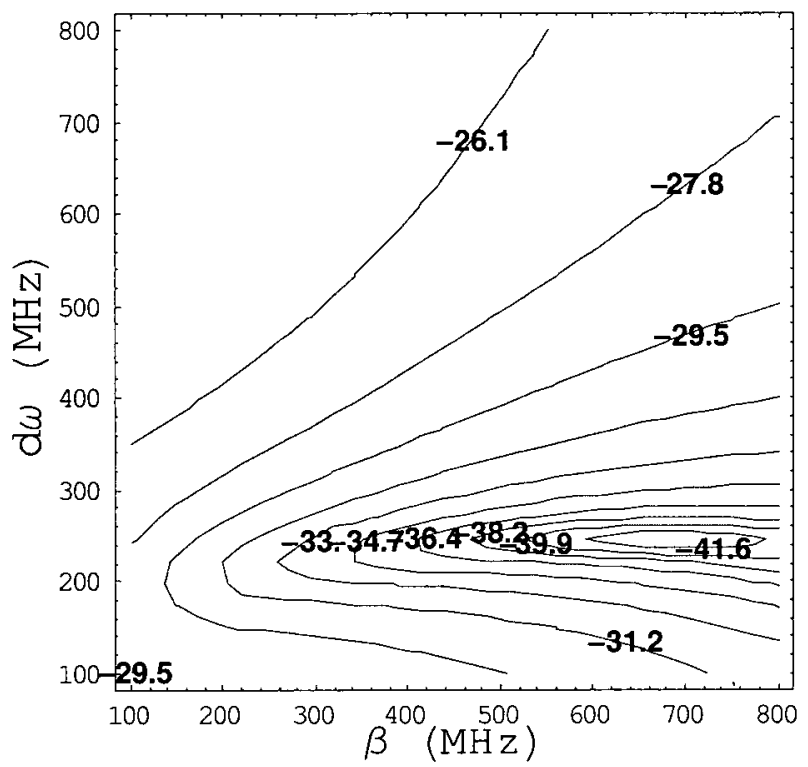

(a)

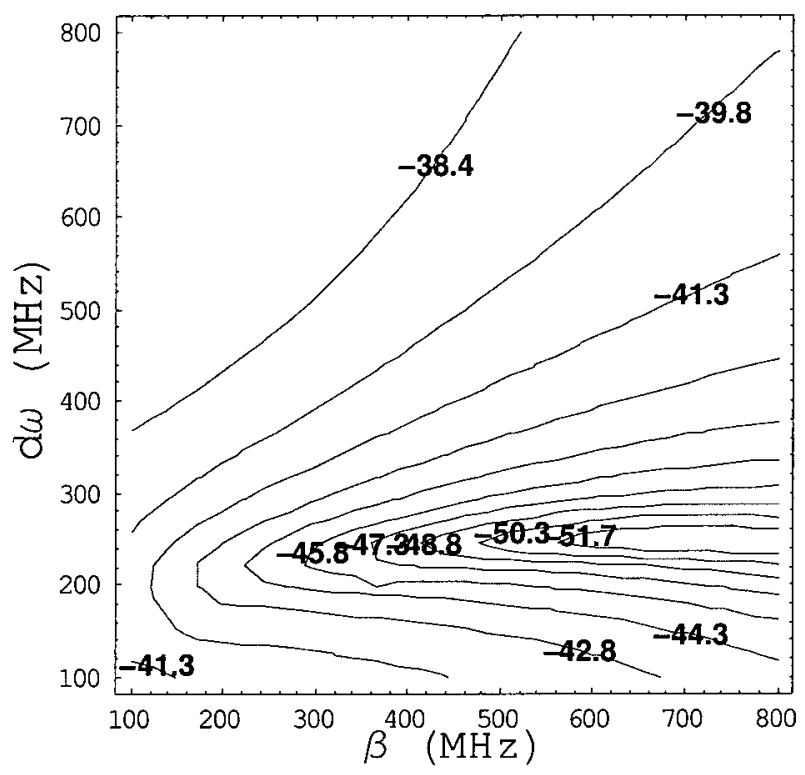

(b)

Figure 4.21: Contour plot of mismatch induced distortion using 1 ns delay: (a) Optical intensity of the $\mathrm{HD}_{2}$ distortion and (b) the IM3 distortion in $\mathrm{dBm}$. 
$\mathrm{HD} 2(\mathrm{dBc})$

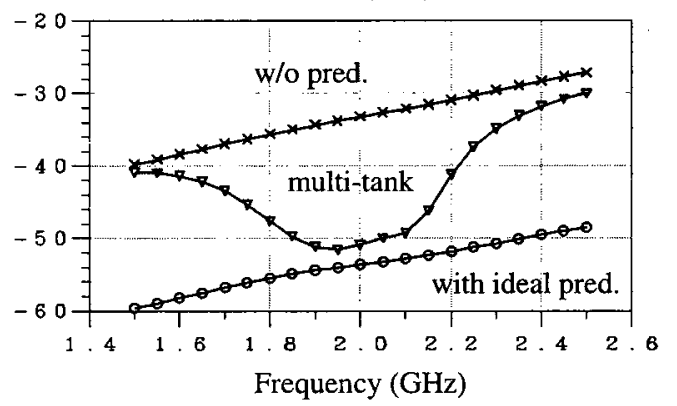

(a)

IM2 $(\mathrm{dBc})$

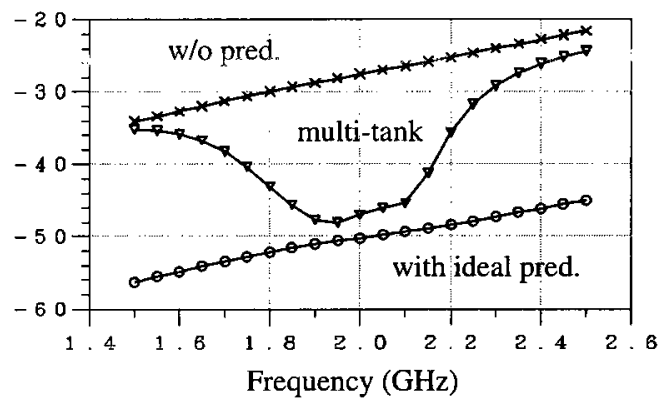

(c)

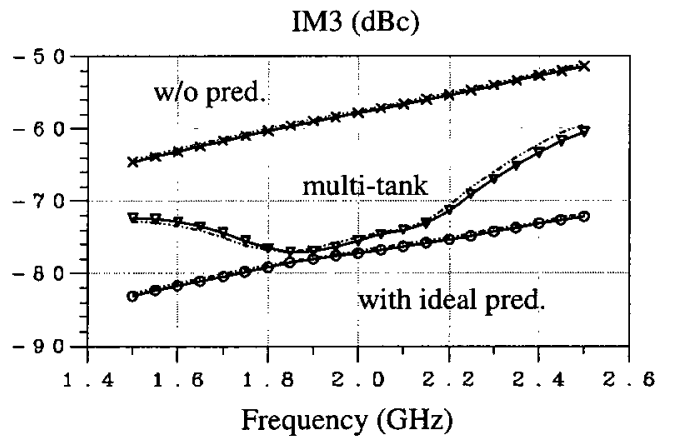

(e)
$\mathrm{HD} 2(\mathrm{dBm})$

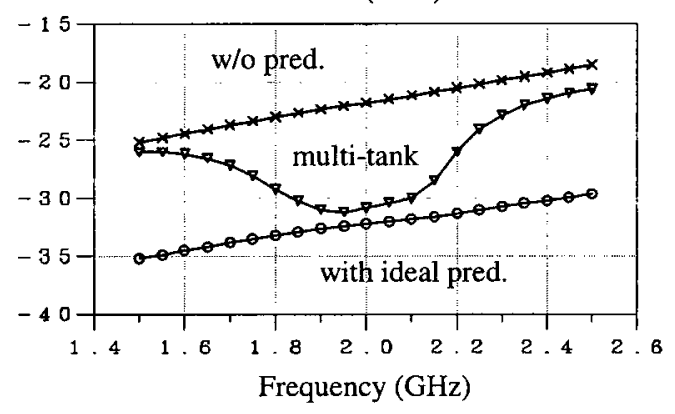

(b)

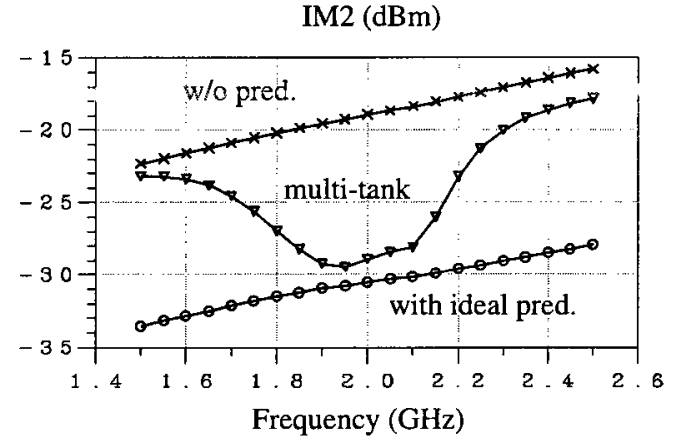

(d)

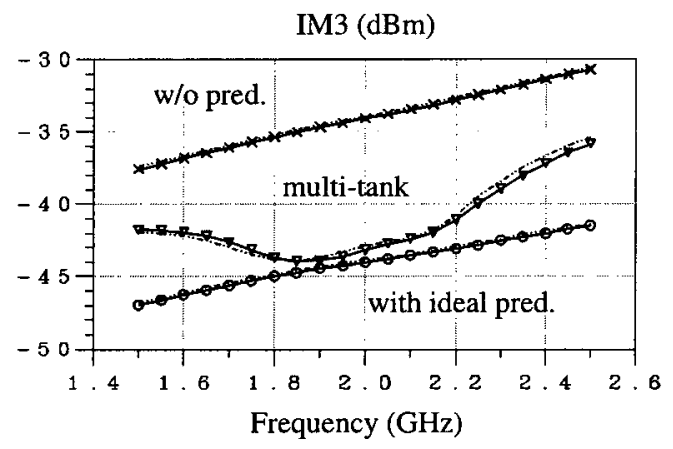

(f)

Figure 4.22: Simulation results of using ideal tank circuits compared with the results of using an ideal predistorter. 


\subsubsection{Overall Circuit and Simulation Results}

The complete circuit of the designed predistorter is shown in Figure 4.23. Four tanks are used for each frequency band (HD2 and IM3 band). The predistorter circuit is implemented in Agilent Advanced Design System (ADS). The first stage is the $X^{2}$ circuit, which is basically a common-source configuration with resistive load and inductive peaking. An extra common-source circuit without the peaking inductor is also placed in the first stage to provide the reference voltage for the next stage. The output voltage of the $X^{2}$ circuit and the reference voltage will drive the differential circuits of the second stage, which are Gilbert cells with LC tanks as the load. Depending on the bias current, the second stage serves as either tunable linear gain cell or tunable cubic cell. Each output of the second stage drive a laser driver. The differential outputs from the laser drivers are connected together to combine the currents. The bias-T networks are used to provide the power supply of the laser drivers. The RF differential driving current from the predistorter is converted to a single-ended output by a wideband balun. The functionality of the balun is to combine the power of the differential outputs, hence canceling the even-mode distortions. It provides $50 \Omega$ for both differential-mode and common-mode signals. The single-ended output of the balun will drive a $50 \Omega$ laser module or connect to a $50 \Omega$ measurement system.

The tuning voltages of the Gilbert cells are obtained using the simulation tool's builtin optimization routine in a two-tone test setup. Figure 4.24 a shows the simulated HD2 distortions $\left(2 f_{2}\right.$ and $\left.2 f_{2}\right)$ of pre-distortion compensated laser (triangle) compared with those of the uncompensated laser (cross). In the frequency range from 1.85-2.15 GHz, more than $15 \mathrm{~dB}$ distortion reduction can be observed for the $2^{\text {nd }}$ order harmonic distortion. By sweeping the input power, HD2 distortion versus output optical intensity from $-14 \mathrm{dBm}$ to $-2 \mathrm{dBm}$ is obtained and shown in Figure $4.24 \mathrm{~b}$. Figure $4.24 \mathrm{c}$ shows the simulated IM2 distortion $\left(f_{1}+f_{2}\right)$ of pre-distortion compensated laser (triangle) compared with those of the uncompensated laser (cross). In the frequency range from $1.85-2.15 \mathrm{GHz}$, more than $10 \mathrm{~dB}$ distortion reduction can be observed. The IM2 distortion versus the output optical intensity is shown in Figure $4.24 \mathrm{~d}$. Figure 4.24 e shows the simulated IM3 distortion 


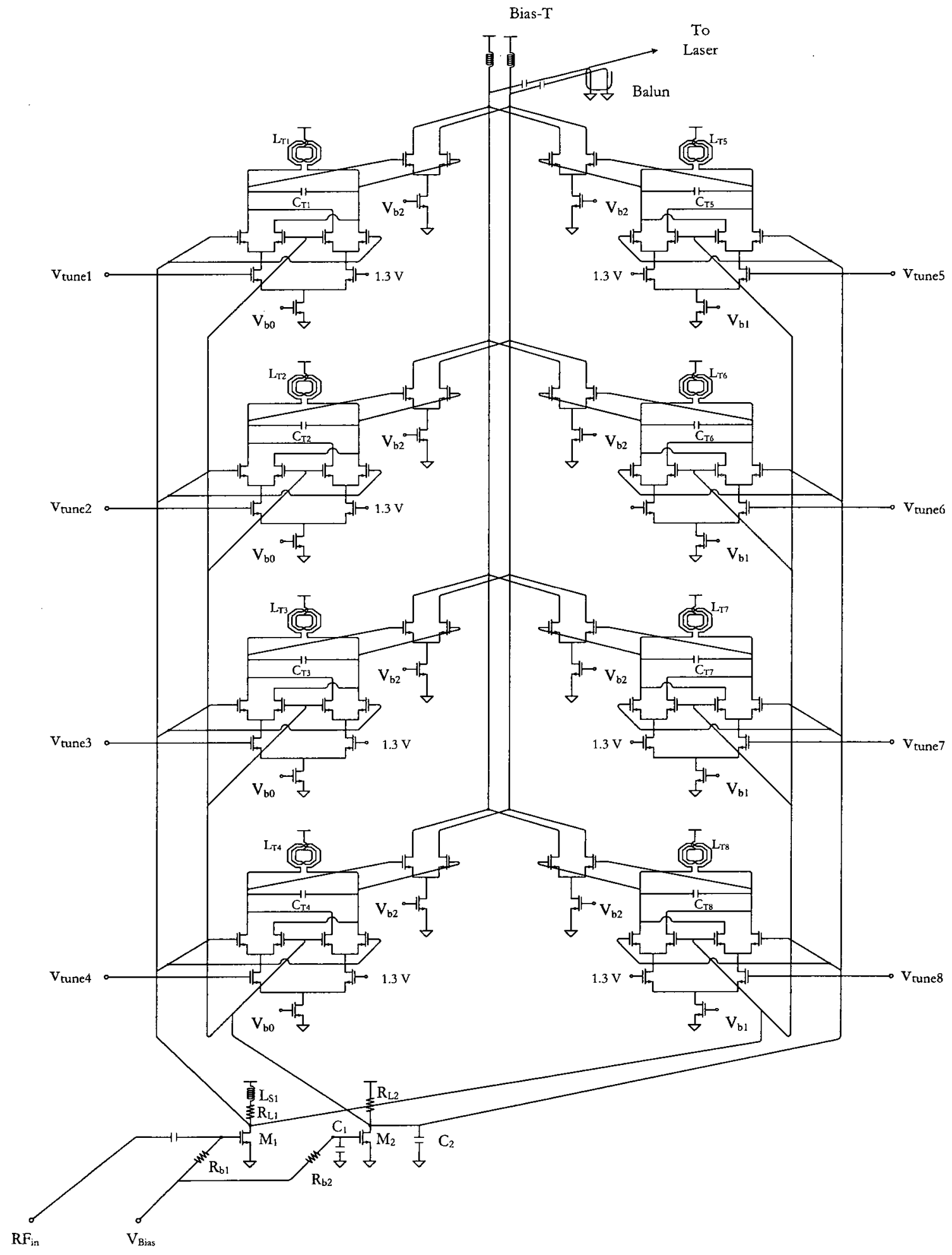

Figure 4.23: Overall circuit (with 4 tank circuits in the IM3 band and 4 tank circuits in the HD2 band). 
$\left(2 f_{1}-f_{2}\right.$ and $\left.2 f_{2}-f_{1}\right)$ of pre-distortion compensated laser (triangle) compared with those of the uncompensated laser (cross). In the frequency range from $1.85-2.15 \mathrm{GHz}$, more than $15 \mathrm{~dB}$ distortion reduction can be observed. The IM3 distortion versus the output optical intensity is shown in Figure $4.24 \mathrm{f}$. 
$\mathrm{HD} 2(\mathrm{dBc})$

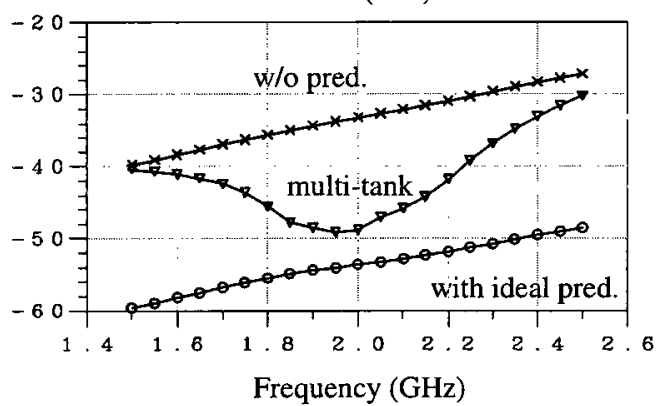

(a)

IM2 (dBc)

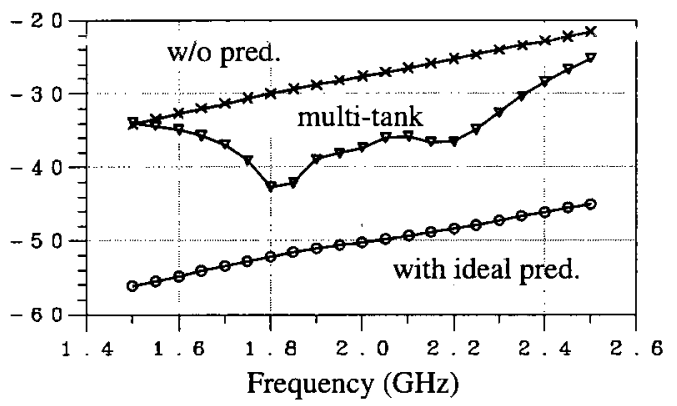

(c)

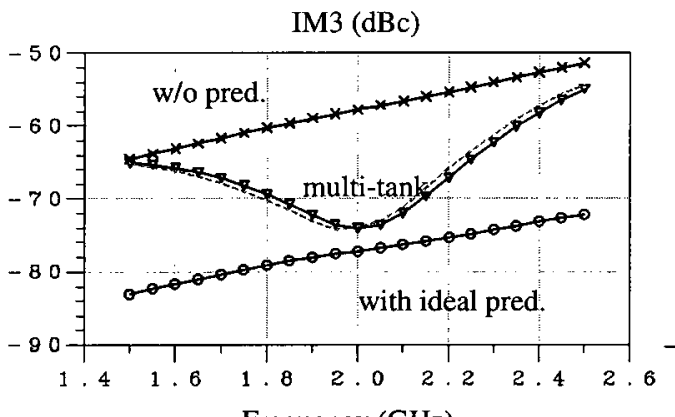

Frequency $(\mathrm{GHz})$

(e)
HD2 (dBc)

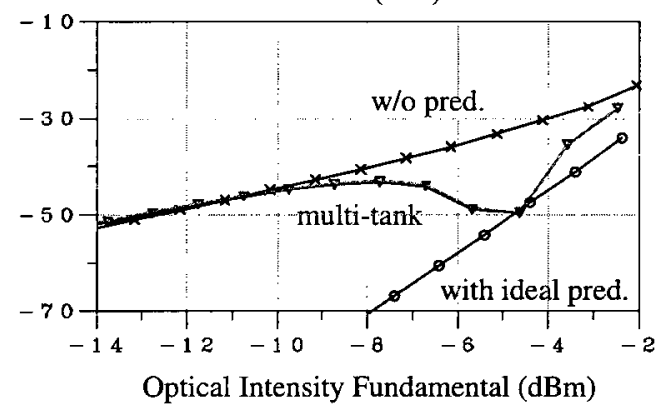

(b)

$\mathrm{IM} 2(\mathrm{dBc})$

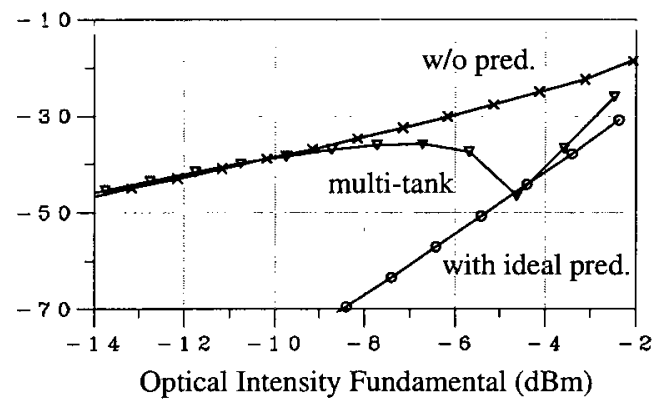

(d)

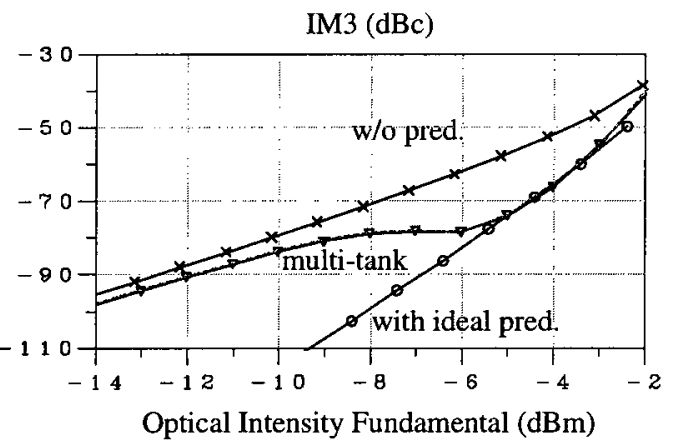

(f)

Figure 4.24: Circuit simulation results. 


\subsection{Design Methodology}

In this section, the systematic approach of designing a multi-tank predistorter will be summarized. Figure 4.25 shows the design flow chart, following which a step-by-step design procedure can be described as follows:

Step 1: Predistorter Characterization

- Laser model parameters extraction from measurements.

- Based on the laser model parameters, calculate to get the predistorter model.

- Using the predistorter model, perform a behavioral level simulation.

If the distortion specifications can not be met by using the ideal predistorter, either increasing laser bias current to lower down the distortion level or relaxing the requirement of maximum output power (in another word, back-off) to reduce the signal-to-distortion ratio.

Step2: Multiple Tank Approximation

- Identifying the frequency band of interest and using multiple tank circuits to approximate the frequency profile shaping filters.

- Designing circuit building blocks. From the behavioral simulation results of Step 1 , for a given linear output, the outputs in each frequency band can be obtained. Given the input signal swing, the total gain for each path and each frequency band can be obtained. This total gain should be properly arranged among the distortion generators, the tank circuit impedances and the gain of the laser driver. The gain arrangement among this stages involves the trad-offs among the circuits' operating bandwidth, tank circuit implementation and the size and the bias current of the laser drivers.

- Obtaining behavioral model of each circuit building block.

- Based on the behavioral models of the circuit building blocks, weighting coefficients of tank circuits can be obtained. Therefore, tank circuits' parameters ( $3 \mathrm{~dB}$ bandwidth and center frequency spacing or inductor and capacitor values) can optimized, for a given linear path delay, to maximize the reduction of distortions. 


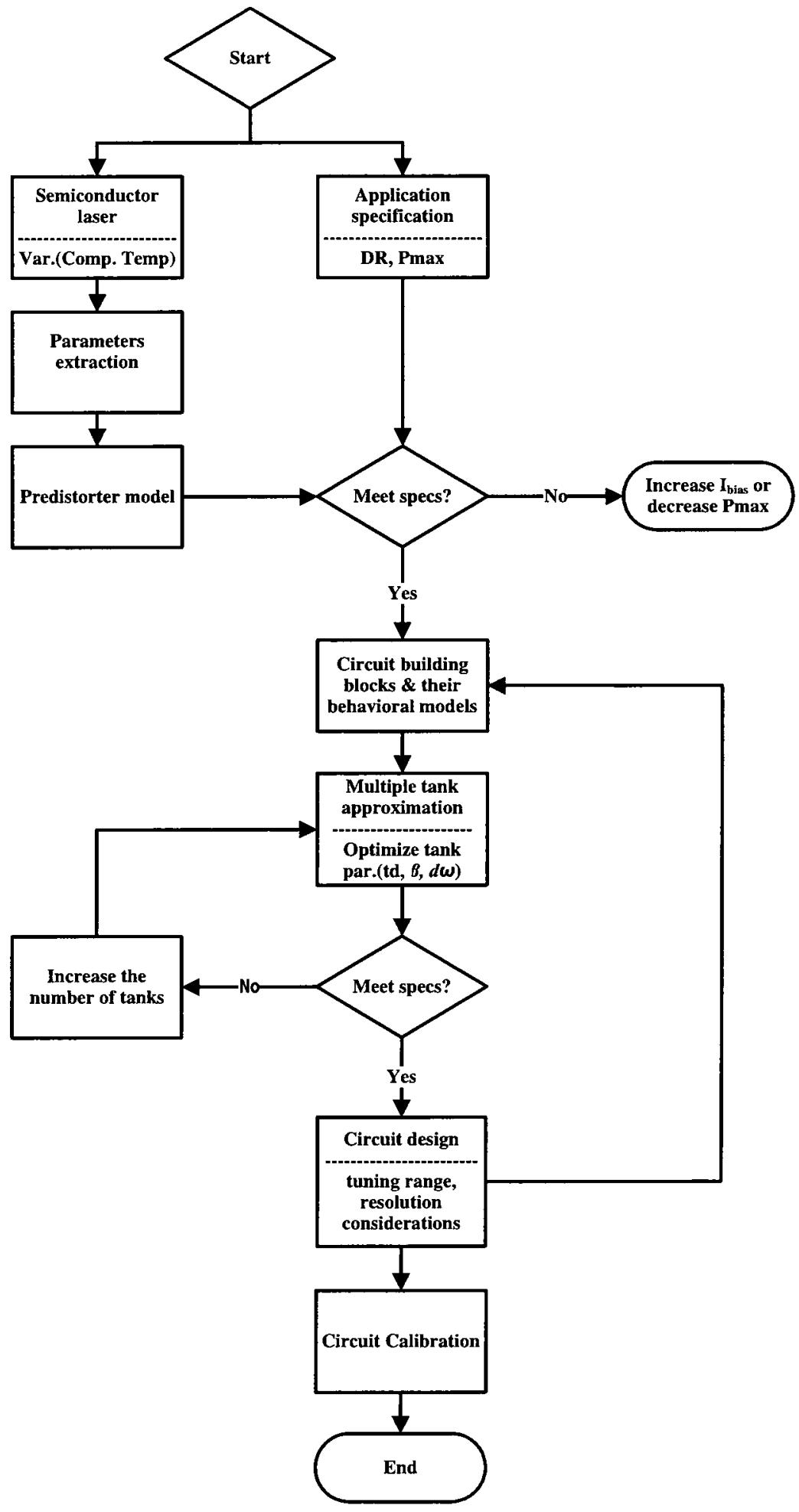

Figure 4.25: Design flow chart. 
If the distortion specifications can not be met for the required frequency range, increase the number of tank circuits.

- Using the multi-tank approximated model, perform a behavioral level simulation.

If the distortion specifications can not be met for the required frequency range, increase the number of tank circuits.

\section{Step3: Complete Circuit Design}

- Assigning gain to each circuit block. The gain tuning resolution corresponds to the specification for the minimum distortion. The maximum gain corresponds to the tuning range (in another word, the maximum distortion to be corrected).

\section{Step4: Circuit Calibration}

- The pre-distortion circuit is by nature sensitive, a good match to the laser is required. Therefore, post-design calibration is necessary. In simulation, it is simply done by using the simulation tool's built-in optimization routine. During measurement, similar optimization routine can be performed by using analog to digital convertors and a Digital Signal Processing (DSP) unit. However, this method is too complicated for circuit implementation. An adaptive calibration using multiple variable feedback is proposed and will be discussed in Chapter 6. This adaptive calibration uses direct feedback, not optimization methods. Therefore, it does not require analogueto-digital converters, data storing memories and DSP unit. The calibration circuit will be a simple analogue implementation.

\subsection{Summary}

In this chapter, circuit level implementation of each building block of the proposed predistorter has been described. A systematic design approach is summarized. Following the step-by-step procedure, a predistorter example has been designed and a satisfactory performance has been obtained in simulation. 


\section{Chapter 5}

\section{Wideband Balun for Even-mode Distortion Cancelation}

The previous chapter describes the design of an prototype integrated CMOS predistortion circuit, which is a device with single-ended input and differential output. However, the laser diode is a single-ended device. A balun is required to convert the differential output of the predistorter to the single-ended input of the laser diode. The usage of a balun offers advantages: 1) increases the output to the laser by combining powers from both of the differential terminals; 2) improves the linearity by reducing even-mode distortions and improving common-mode interference rejection.

As discussed earlier, the predistorter's output spectrum contains several frequency bands. This chapter presents the design technique of a wideband balun to cover the frequency bands of the predistortion signal. The proposed balun structure is based on the basic Marchand balun, for its excellent performance on opposite-phase and equalamplitude.

\subsection{Basic Marchand Balun}

The upper part of Figure 5.1 shows the basic Marchand balun structures in the coaxial form $[1,66]$. The Marchand baluns basically consist of an unbalanced $\left(Z_{a}\right)$ and a shortcircuited $\left(Z_{a b}\right)$ section in the uncompensated balun and an extra open-circuited section $\left(Z_{b}\right)$ in the compensated balun. Their equivalent transmission-line models are shown at 

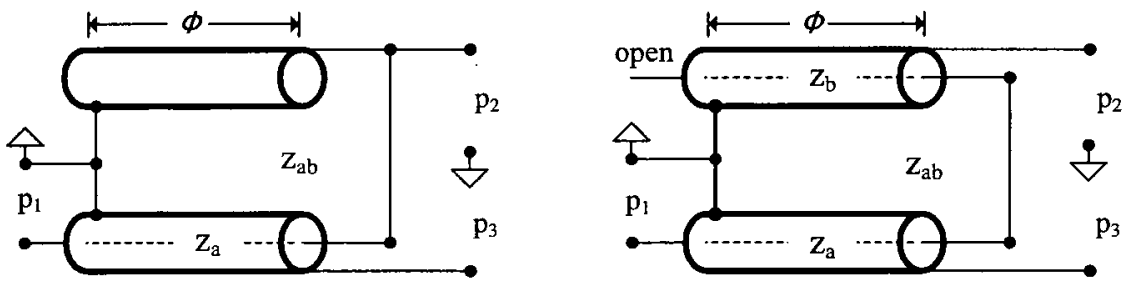

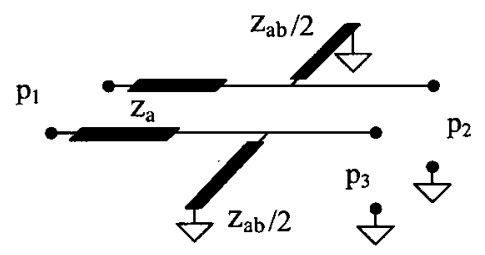

(a)

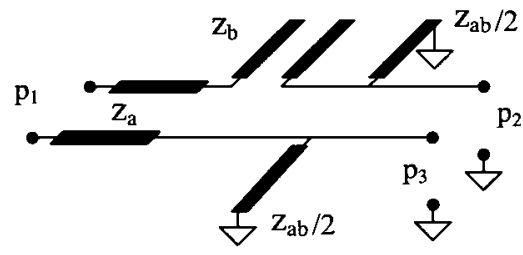

(b)

Figure 5.1: Basic (a) uncompensated and (b) compensated Marchand balun and their equivalent circuits.

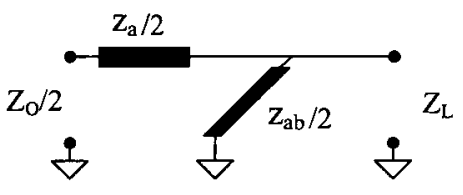

(a)

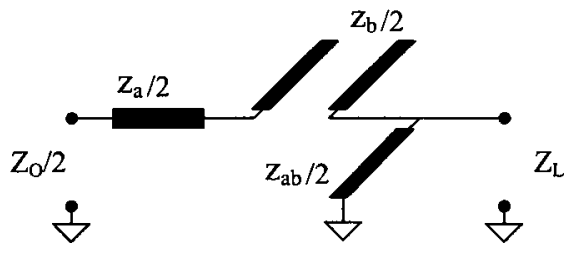

(b)

Figure 5.2: Bisected equivalent circuits of (a) uncompensated and (b) compensated Marchand balun for differential mode operation. 
the lower part of Figure 5.1. The short stubs $\left(Z_{a b} / 2\right)$ represent the impedance of the outer shield with respect to the ground.

There are two short-stubs $\left(Z_{a b} / 2\right)$, one is associated with $Z_{a}$, the other is purposely introduced to symmetrize the equivalent circuit. It is this symmetry gives Marchand baluns superior performance on opposite-phase and equal-amplitude of the balanced output. The bisected equivalent circuits of the Marchand baluns for the odd-mode operation are shown in Figure 5.2. Referring to this, the equivalent impedance looking into the balun from the unbalanced port is

$$
Z_{\text {in }}=\frac{Z_{a}}{2} \frac{Z_{T}+\frac{Z_{a}}{2} S}{\frac{Z_{a}}{2}+Z_{T} S}
$$

where, for the uncompensated structure

$$
Z_{T}=\frac{1}{2} Z_{a b} S / / Z_{L}
$$

and for the compensated structure

$$
Z_{T}=\frac{1}{2} Z_{b} / S+\frac{1}{2} Z_{a b} S / / Z_{L}
$$

and $\mathrm{S}$ is the Richard's transformation of

$$
S=j \cdot \operatorname{Tan}(\phi)=j \cdot \operatorname{Tan}\left(\frac{\pi}{2} \frac{f}{f_{0}}\right) .
$$

$\phi$ is the electrical length of the transmission-line elements, $f_{0}$ is the frequency for which the transmission-lines are a quarter wavelength long. For a wideband matching with maximum flat property,

$$
\left.Z_{i n}\right|_{f=f_{0}}=\frac{Z_{o}}{2}
$$

and

$$
\left.\frac{\partial Z_{i n}}{\partial f}\right|_{f=f_{0}}=0
$$

should be satisfied for the uncompensated case, and the second order derivative is obtained as

$$
\left.\frac{\partial^{2} Z_{i n}}{\partial f^{2}}\right|_{f=f_{0}}=4 \frac{Z_{L}^{2}}{Z_{a b}}\left(\frac{Z_{L}}{Z_{a b}}+\sqrt{1+\frac{Z_{L}^{2}}{Z_{a b}}}\right)^{3}
$$


and

$$
\begin{aligned}
& \left.Z_{i n}\right|_{f=f_{0}}=\frac{Z_{o}}{2} \\
& \left.\frac{\partial Z_{i n}}{\partial f}\right|_{f=f_{0}}=0 \\
& \left.\frac{\partial^{2} Z_{i n}}{\partial f^{2}}\right|_{f=f_{0}}=0
\end{aligned}
$$

should be satisfied for the compensated case, and the third order derivative is obtained as

$$
\left.\frac{\partial^{3} Z_{i n}}{\partial f^{3}}\right|_{f=f_{0}}=-12 j\left(\frac{1}{\frac{Z_{a b}}{Z_{L}^{2}} \sqrt{\frac{Z_{a b}^{2}}{4 Z_{L}^{2}}+1}}+\frac{1}{\frac{Z_{a b}}{Z_{L}^{2}}\left(\frac{Z_{a b}^{2}}{4 Z_{L}^{2}}+1\right)}\right)
$$

Equations (5.1.7) and (5.1.11) describe the deviation from the ideal flat response, therefore, they should be kept close to zero for a wideband flat response. It can be seen from the expressions, in order to minimize the value, a small value of $Z_{L}$ and a large value of $Z_{a b}$ are desired for a wideband impedance matching. While $Z_{a b}$ is usually limited in a fabrication process due to the large transmission-line width used for $Z_{a b}$ and the radiation resistance of the transmission-line, a natural step is to incorporate impedance transformers into the existing balun structures in order to transform the high load impedance to a desired low impedance and to generate a higher-order filter response.

\subsection{Wideband Marchand Balun}

In order to achieve a higher order filter response, the balun structures incorporated with stepped impedance transformers are expanded into ladder-network configurations. All the possibilities are listed in Figure 5.3. Instead of allowing the series-open element to float, it would be more practical to have its shielding connected to the ground. In this way, there is always a shunt-short element associated with the series-open element. Figure 5.3 (a) is the uncompensated balun with the stepped impedance transformer. Figure 5.3 (c) is the compensated balun with the stepped impedance transformer. Figure 5.3 (e) can be viewed as a cascade of multiple compensated structures with the step transformer. Figure 5.3 (b) and (d) are structures with redundancy, which are not recommended. Even though 

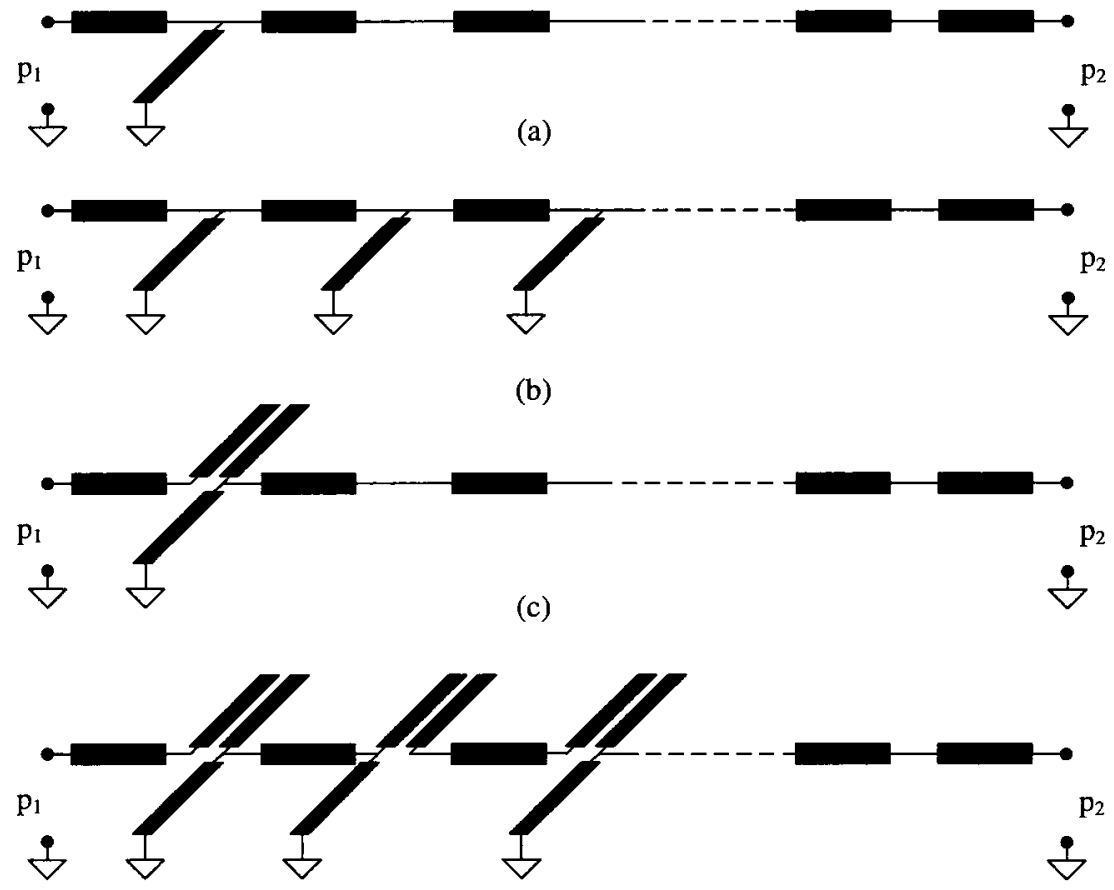

(d)

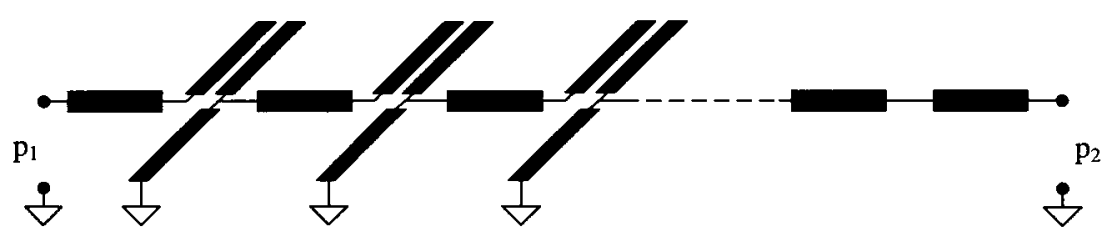

(e)

Figure 5.3: Possible balun configurations using open or short stubs and unit elements (b and $d$ are topologies with redundancy). 
Figure 5.3 (b) is a commonly used structure for a bandpass filter, but for wideband baluns the structure will potentially reduce the available bandwidth. As illustrated in Figure 5.4, through the equivalency of Kuroda identities [67], a shunt-short stub can be transferred to the location of the first short stub. This leaves a transformer at the original place. As an ideal transformer is independent of frequency, the transformer will not affect the order of the filter response. As the transferred short stub will be combined with the first short stub, the overall impedance is effectively reduced. As concluded in the previous section, a large impedance is preferred for a wide bandwidth design. This redundant short stub will apparently reduce the available bandwidth. Similar reasoning applies to structure "d". Therefore, the series-open and the shunt-short should be placed alternatively as in Figure 5.3 (e) to avoid redundancy.

Figure 5.5 lists the topologies of the proposed multiple stage Marchand balun in the ascending order of the number of short or open stubs used. To generalize, the stepped impedance transformer is included as the case of zero order. The uncompensated balun is the case of the first order. The compensated balun is the case of the second order. Then an uncompensated structure followed by a compensated structure is the third order, and so on.
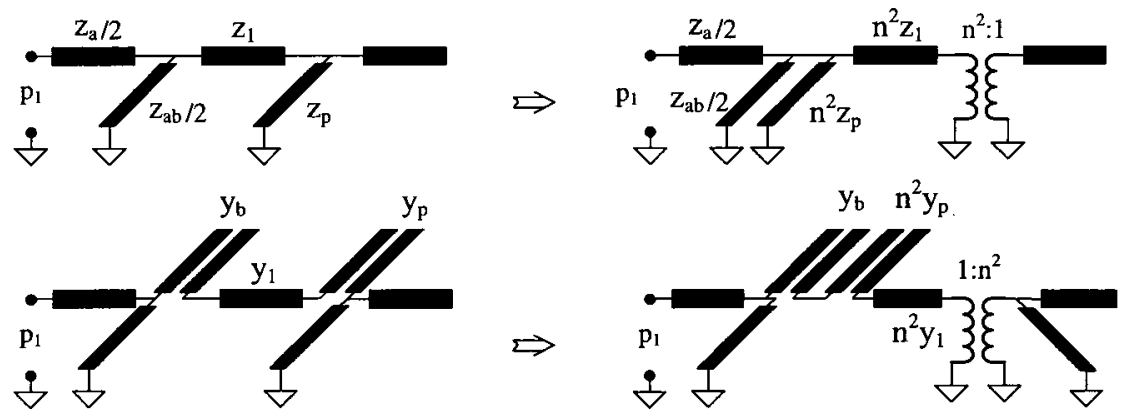

Figure 5.4: Redundancy illustrated by the equivalency of Kuroda identities.

\subsubsection{Derivation of Design Equations}

To obtain the circuit transfer function, chain-scattering matrices are used. The overall chain-scattering matrix can be obtained by multiplying the chain-scattering matrices of 

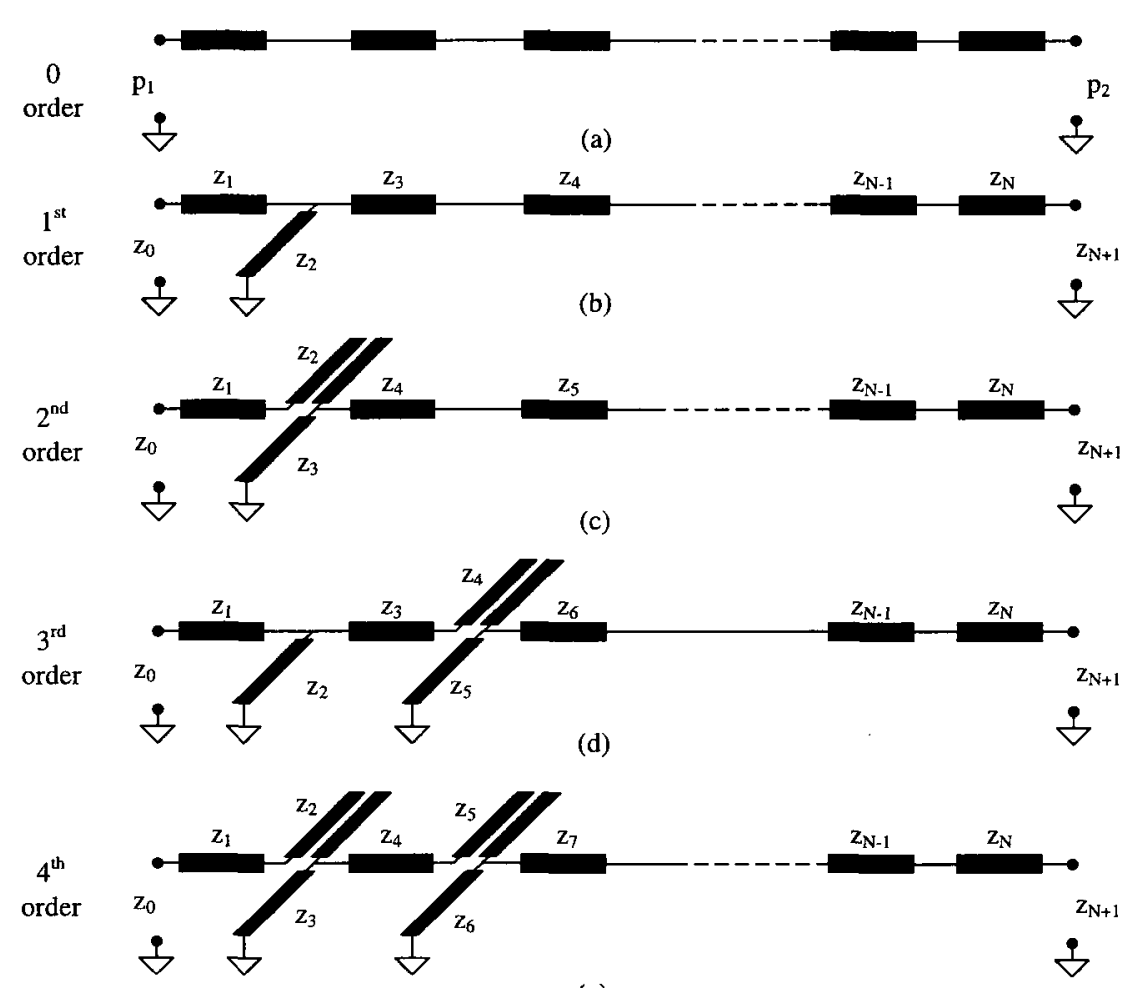

(e)

Figure 5.5: Topologies of the proposed multiple stage Marchand balun in the ascending sequence of warping orders (number of short or open stubs). 
the individual elements in the balun ladder network

$$
\left[\begin{array}{ll}
t_{11} & t_{12} \\
t_{21} & t_{22}
\end{array}\right]_{\text {overall }}=\prod_{i=1}^{N}\left[\begin{array}{ll}
t_{11}^{i} & t_{12}^{i} \\
t_{21}^{i} & t_{22}^{i}
\end{array}\right]
$$

where the chain scattering parameters $t_{11}$ and $t_{21}$ are related to the reflection coefficient $S_{11}$ and the transmission coefficient $S_{21}$ by

$$
\begin{aligned}
& S_{11}=\frac{t_{21}}{t_{11}} \\
& S_{21}=\frac{1}{t_{11}}
\end{aligned}
$$

For a lossless reciprocal network, we have the property of

$$
\left|t_{11}\right|^{2}-\left|t_{21}\right|^{2}=1
$$

With (5.2.2), (5.2.3) and (5.2.4), we obtain

$$
\begin{aligned}
& \left|S_{11}\right|^{2}=\frac{1}{1+\frac{1}{\left|t_{21}\right|^{2}}} \\
& \left|S_{21}\right|^{2}=\frac{1}{1+\left|t_{21}\right|^{2}}
\end{aligned}
$$

From (5.2.5) and (5.2.6), it can be seen that, when $t_{21}$ is made equal-ripple, $S_{11}$ and $S_{21}$ are also equal-ripple.

The basic quarter-wave elements used for Marchand balun include series-unit, shuntshort and series-open transmission-line sections. Their chain scattering matrices are listed in Table 5.1. $\phi$ in these matrices is the electrical length of the quarter-wave elements and is defined as

$$
\phi=\frac{\pi f}{2 f_{o}}
$$

where $f_{o}$ is the frequency for which the transmission lines are a quarter wavelength long. In these matrices, $t_{11}, t_{12}, t_{21}$, and $t_{22}$ are fractions of rational polynomials of $e^{2 j \phi}$ with the numerator in the form of

$$
c_{0}^{(l, m)}+c_{1}^{(l, m)} e^{2 j \phi}
$$


Table 5.1: Basic quarter-wave transmission line elements and their chain scattering parameters

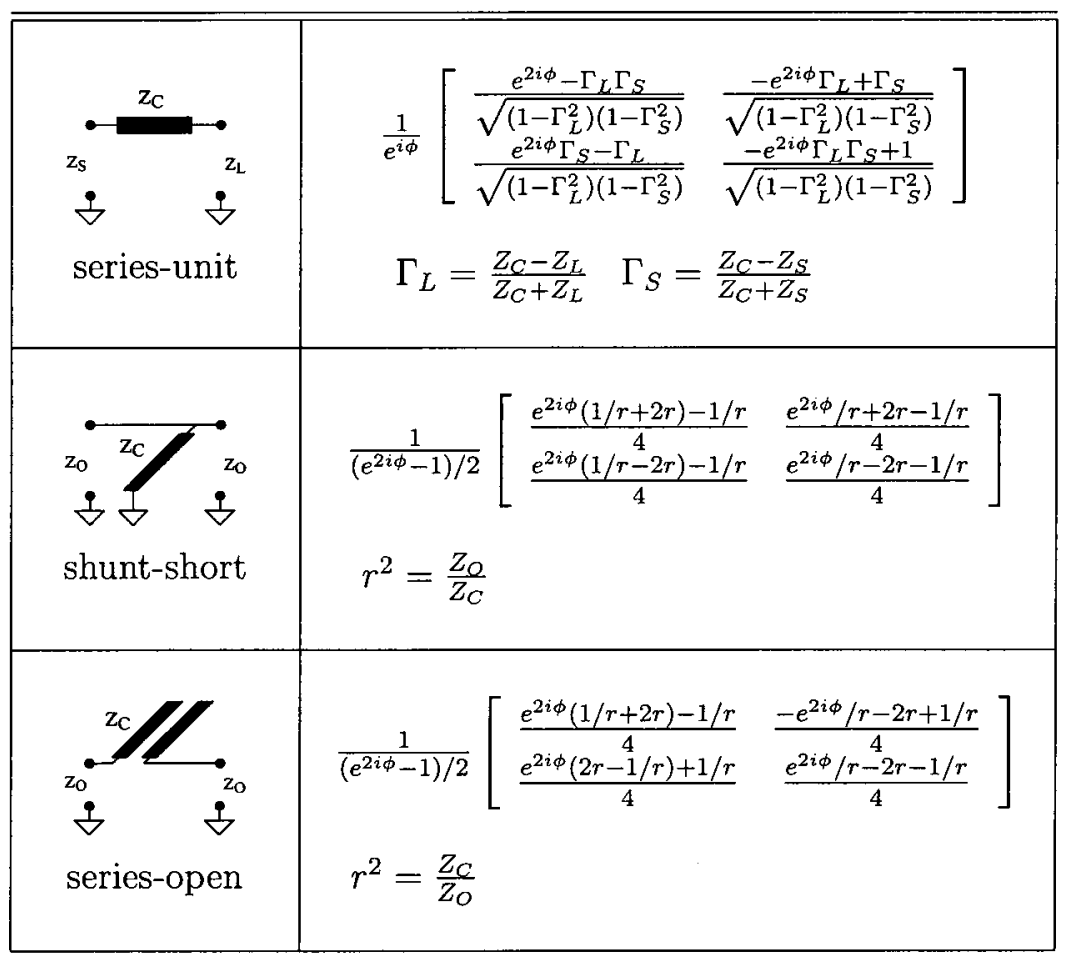


where the superscript $(l, m)$ is used to identify $t_{11}, t_{12}, t_{21}$ or $t_{22}$. The denominator of the series-unit element is a time-delay unit $\left(e^{j \phi}\right)$. The denominators of the shunt-short and the series-open elements are the same, and represent a dc pole in the form of $\frac{1}{2}\left(e^{2 j \phi}-1\right)$.

The overall T-matrix of the first order Marchand balun using $N$ quarter-wave elements, can be represented by a sequential multiplication

$$
T_{\text {overall }}^{1 \text { st }}=\left.\left.\left.\left.\left.T_{\text {unit }}^{(1)}\right|_{\substack{\Gamma_{S}=\Gamma_{1} \\ \Gamma_{L}=0}} \cdot T_{\text {short }}^{(2)}\right|_{r=r s_{2}} \cdot T_{\text {unit }}^{(3)}\right|_{\substack{\Gamma_{S}=\Gamma_{3} \\ \Gamma_{L}=0}} \cdot T_{\text {unit }}^{(4)}\right|_{\Gamma_{L}=\Gamma_{4}=0} \cdot \ldots \cdot T_{\text {unit }}^{(N)}\right|_{\substack{\Gamma_{S}=\Gamma_{N}=-\Gamma_{N+1} \\ \Gamma_{N}}}
$$

where

$$
\Gamma_{i}=\frac{z_{i}-z_{i-1}}{z_{i}+z_{i-1}} \quad \text { and } \quad r s_{i}^{2}=\frac{z_{i-1}}{z_{i}} .
$$

Similarly, the overall T-matrix of the second order Marchand balun can be obtained as

$$
T_{\text {overall }}^{2 n d}=T_{\text {unit }}^{(1)} \cdot T_{\text {open }}^{(2)} \cdot T_{\text {short }}^{(3)} \cdot T_{\text {unit }}^{(4)} \cdot \ldots \cdot T_{\text {unit }}^{(N)}
$$

and the overall T-matrix of the third order Marchand balun is

$$
T_{\text {overall }}^{3 r d}=T_{\text {unit }}^{(1)} \cdot T_{\text {short }}^{(2)} \cdot T_{\text {unit }}^{(3)} \cdot T_{\text {open }}^{(4)} \cdot T_{\text {short }}^{(5)} \ldots T_{\text {unit }}^{(N)}
$$

and so on.

Considering (5.3.6) (5.3.7) and (5.3.8), the elements in the overall T-matrix for a $k^{\text {th }}$ order $N$-element Marchand balun would be in the form of

$$
T_{\text {overall }}^{k_{t h}}=\left[\begin{array}{cc}
t_{11}^{k_{t h}} & t_{12}^{k_{t h}} \\
t_{21}^{k_{t h}} & t_{22}^{k_{t h}}
\end{array}\right]
$$

where,

$$
t_{(l, m)}^{k_{t h}}=\frac{\sum_{n=0}^{N} A_{n}(l, m) \cdot e^{2 n j \phi}}{e^{j(N-k) \phi}\left(\frac{e^{2 j \phi}-1}{2}\right)^{k}}=\frac{\sum_{n=0}^{N} A_{n}(l, m) \cdot e^{2 n j \phi}}{e^{j N \phi} \cdot \sin (\phi)^{k}}
$$

In order to make $t_{21}$ equal-ripple, $k$ dc poles in the denominator of (5.3.11) must be considered. A simple way would be generating the same number of dc zeros to cancel these poles. However, redundancy is produced in this way due to the reduction of the transfer function order. Therefore, the optimal performance out of this balun structure would not be obtained. From equation (5.3.11), it can be seen that any shape of frequency 
response obtained by the numerator polynomial will be warped by the inverse of $\sin (\phi)^{k}$. Therefore, if we "pre-warp" the numerator's frequency response into a ripple shape with an envelope of the $k^{\text {th }}$ power of the sinusoidal, we would have the desired equal-ripple result, as illustrated in Figure 5.6.
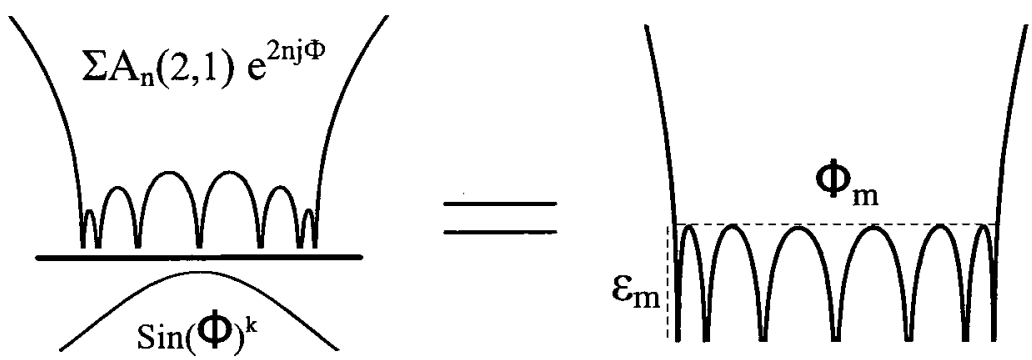

Figure 5.6: Prewarping to obtain an equal-ripple response.

The design polynomials for these warped ripple shapes are defined as

$$
p_{N}(x)= \begin{cases}\sum_{n=0}^{N / 2} c_{n} \cdot x^{2 n}, & N \in \text { even } \\ \sum_{n=1}^{(N+1) / 2} c_{n} \cdot x^{2 n-1}, & N \in \text { odd }\end{cases}
$$

The coefficients $c_{n}$ can be obtained by solving the equations of

$$
p_{N}\left(x_{i}\right)=(-1)^{i} \sin \left(\cos ^{-1}\left(x_{i} q\right)\right)^{k}
$$

where $i$ is from 1 to $N / 2$ for an even number $N$ or from 1 to $(N-1) / 2$ for an odd number $N$, and $x_{i} s$ are \pm 1 and the roots of

$$
\partial_{x}\left(\frac{p_{N}(x)}{\sin \left(\cos ^{-1}(x q)\right)^{k}}\right)=0 .
$$

To save the computation effort, a closed form of (5.3.4)

$$
\partial_{x} p_{N}(x)=0
$$

is used instead, where $q$ is the bandwidth index and related to the equal-ripple bandwidth $\phi_{m}$ by

$$
\phi_{m}=\pi-2 \cos ^{-1}(q)
$$


$q$ can be obtained through the balun's bandwidth ratio $B_{r}$ by

$$
q=\cos \left(\frac{\pi}{1+B_{r}}\right)
$$

The calculated coefficients of the design polynomials are listed in Table 5.2, 5.3, 5.4, and 5.5 for $k$ equal to $1,2,3$ and 4 respectively, where coefficients $c_{n}$ are functions of the bandwidth index $q$ and are represented as the power series expansion of $q$ for up to a fifth order

$$
c_{i}=e_{0}+e_{1} q^{2}+e_{2} q^{4}+e_{3} q^{6}+e_{4} q^{8}+e_{5} q^{10} .
$$

Once the design polynomial is obtained, it will be expanded as a polynomial of $e^{2 n j \phi}$

Table 5.2: Design polynomials for $1^{\text {st }}$ order pre-warping.

\begin{tabular}{|r|r|r|r|r|r|r|r|}
\hline $\mathrm{N}$ & $1^{\text {st }}$ & $e_{0}$ & $e_{1}$ & $e_{2}$ & $e_{3}$ & $e_{4}$ & $e_{5}$ \\
\hline \hline \multirow{4}{*}{4} & $c_{0}$ & 1 & & & & & \\
\cline { 2 - 8 } & $c_{1}$ & -8 & 1.5 & 0.3438 & 0.1641 & 0.0997 & 0.0686 \\
\cline { 2 - 8 } & $c_{2}$ & 8 & -2 & -0.4688 & -0.2266 & -0.1388 & -0.0959 \\
\hline \multirow{4}{*}{5} & $c_{1}$ & 5 & -0.5 & -0.115 & -0.0569 & -0.035 & -0.0241 \\
\cline { 2 - 9 } & $c_{2}$ & -20 & 4 & 0.95 & 0.4644 & 0.2842 & 0.1955 \\
\cline { 2 - 8 } & $c_{3}$ & 16 & -4 & -0.96 & -0.47 & -0.2883 & -0.1988 \\
\hline \multirow{4}{*}{6} & $c_{0}$ & -1 & & & & & \\
\cline { 2 - 8 } & $c_{1}$ & 18 & -2.5 & -0.5972 & -0.2944 & -0.1818 & -0.1258 \\
\cline { 2 - 8 } & $c_{2}$ & -48 & 10 & 2.4167 & 1.1902 & 0.734 & 0.5074 \\
\cline { 2 - 8 } & $c_{3}$ & 32 & -8 & -1.9444 & -0.9583 & -0.5912 & -0.409 \\
\hline \multirow{4}{*}{8} & $c_{1}$ & -7 & 0.5 & 0.1199 & 0.0597 & 0.037 & 0.0257 \\
\cline { 2 - 8 } & $c_{2}$ & 56 & -9 & -2.1837 & -1.0803 & -0.6688 & -0.4641 \\
\cline { 2 - 8 } & $c_{3}$ & -112 & 24 & 5.8571 & 2.8969 & 1.793 & 1.2439 \\
\cline { 2 - 8 } & $c_{4}$ & 64 & -16 & -3.9184 & -1.9388 & -1.2002 & -0.8328 \\
\hline \multirow{4}{*}{8} & $c_{0}$ & 1 & & & & & \\
\cline { 2 - 8 } & $c_{1}$ & -32 & 3.5 & 0.8516 & 0.4231 & 0.2627 & 0.1827 \\
\cline { 2 - 8 } & $c_{2}$ & 160 & -28 & -6.8516 & -3.3982 & -2.1083 & -1.4659 \\
\cline { 2 - 8 } & $c_{3}$ & -256 & 56 & 13.75 & 6.8189 & 4.2301 & 2.941 \\
\cline { 2 - 8 } & $c_{4}$ & 128 & -32 & -7.875 & -3.9063 & -2.4236 & -1.6852 \\
\hline
\end{tabular}

$$
\varepsilon_{m} \cdot p_{N}\left(\frac{\cos (\phi)}{q}\right)=e^{-j N \phi} \sum_{n=0}^{N} a_{n} e^{2 n j \phi}
$$

where $\varepsilon_{m}$ is the ripple amplitude which is roughly equal to the passband reflection coefficient. Compare (5.2.22) with (5.3.11) and set the corresponding coefficients equal for 
Table 5.3: Design polynomials for $2^{\text {nd }}$ order pre-warping.

\begin{tabular}{|r|r|r|r|r|r|r|r|}
\hline $\mathrm{N}$ & $2^{\text {nd }}$ & $e_{0}$ & $e_{1}$ & $e_{2}$ & $e_{3}$ & $e_{4}$ & $e_{5}$ \\
\hline \hline \multirow{4}{*}{4} & $c_{0}$ & 1 & & & & & \\
\cline { 2 - 8 } & $c_{1}$ & -8 & 3 & 0.3750 & 0.1875 & 0.1113 & 0.0732 \\
\cline { 2 - 8 } & $c_{2}$ & 8 & -4 & -0.3750 & -0.1875 & -0.1113 & -0.0732 \\
\hline \multirow{4}{*}{5} & $c_{1}$ & 5 & -1 & -0.21 & -0.1002 & -0.0603 & -0.0407 \\
\cline { 2 - 8 } & $c_{2}$ & -20 & 8 & 1.05 & 0.5202 & 0.3179 & 0.2166 \\
\cline { 2 - 8 } & $c_{3}$ & 16 & -8 & -0.84 & -0.42 & -0.2576 & -0.1758 \\
\hline \multirow{4}{*}{7} & $c_{0}$ & -1 & & & & & \\
\cline { 2 - 8 } & $c_{1}$ & 18 & -5 & -0.8889 & -0.4383 & -0.2678 & -0.1835 \\
\hline & $c_{2}$ & -48 & 20 & 2.6667 & 1.3272 & 0.8158 & 0.5611 \\
\hline & $c_{3}$ & 32 & -16 & -1.7778 & -0.8889 & -0.548 & -0.3776 \\
\hline \multirow{4}{*}{8} & $c_{1}$ & -7 & 1 & 0.2296 & 0.1127 & 0.0692 & 0.0476 \\
\cline { 2 - 8 } & $c_{2}$ & 56 & -18 & -2.9847 & -1.4819 & -0.9129 & -0.6296 \\
\cline { 2 - 8 } & $c_{3}$ & -112 & 48 & 6.4286 & 3.206 & 1.9797 & 1.3678 \\
\cline { 2 - 8 } & $c_{4}$ & 64 & -32 & -3.6735 & -1.8367 & -1.136 & -0.7858 \\
\hline \multirow{4}{*}{8} & $c_{0}$ & 1 & & & & & \\
\cline { 2 - 8 } & $c_{1}$ & -32 & 7 & 1.4063 & 0.6973 & 0.4304 & 0.2976 \\
\cline { 2 - 8 } & $c_{2}$ & 160 & -56 & -8.9063 & -4.4356 & -2.743 & -1.8990 \\
\hline & $c_{3}$ & -256 & 112 & 15 & 7.4883 & 4.6369 & 3.2134 \\
\cline { 2 - 8 } & $c_{4}$ & 128 & -64 & -7.5 & -3.75 & -2.3243 & -1.6119 \\
\hline
\end{tabular}

Table 5.4: Design polynomials for $3^{\text {rd }}$ order pre-warping.

\begin{tabular}{|r|r|r|r|r|r|r|r|}
\hline $\mathrm{N}$ & $3^{\text {rd }}$ & $e_{0}$ & $e_{1}$ & $e_{2}$ & $e_{3}$ & $e_{4}$ & $e_{5}$ \\
\hline \hline \multirow{4}{*}{5} & $c_{1}$ & 5 & -1.5 & -0.285 & -0.1263 & -0.073 & -0.0481 \\
\cline { 2 - 8 } & $c_{2}$ & -20 & 12 & 0.3 & 0.3488 & 0.2285 & 0.16 \\
\cline { 2 - 8 } & $c_{3}$ & 16 & -12 & 0.36 & -0.16 & -0.1321 & -0.1003 \\
\hline \multirow{4}{*}{6} & $c_{0}$ & -1 & & & & & \\
\cline { 2 - 8 } & $c_{1}$ & 18 & -7.5 & -0.875 & -0.4479 & -0.2739 & -0.1869 \\
\cline { 2 - 8 } & $c_{2}$ & -48 & 30 & 0.75 & 0.8854 & 0.5950 & 0.4224 \\
\cline { 2 - 8 } & $c_{3}$ & 32 & -24 & -0.5 & -0.375 & -0.2977 & -0.2238 \\
\hline \multirow{4}{*}{7} & $c_{1}$ & -7 & 1.5 & 0.3291 & 0.1575 & 0.0954 & 0.0650 \\
\cline { 2 - 8 } & $c_{2}$ & 56 & -27 & -2.4031 & -1.3832 & -0.8680 & -0.602 \\
\hline & $c_{3}$ & -112 & 72 & 1.7143 & 2.1045 & 1.4315 & 1.0256 \\
\hline & $c_{4}$ & 64 & -48 & 0.7347 & -0.8163 & -0.6354 & -0.4768 \\
\hline \multirow{4}{*}{8} & $c_{0}$ & 1 & & & & & \\
\cline { 2 - 8 } & $c_{1}$ & -32 & 10.5 & 1.6641 & 0.8298 & 0.5104 & 0.3515 \\
\hline & $c_{2}$ & 160 & -84 & -6.1641 & -3.8762 & -2.4772 & -1.7356 \\
\cline { 2 - 7 } & $c_{3}$ & -256 & 168 & 3.75 & 4.8276 & 3.3129 & 2.3873 \\
\cline { 2 - 7 } & $c_{4}$ & 128 & -96 & 1.125 & -1.7188 & -1.3226 & -0.9915 \\
\hline
\end{tabular}


Table 5.5: Design polynomials for $4^{\text {th }}$ order pre-warping.

\begin{tabular}{|r|r|r|r|r|r|r|r|}
\hline $\mathrm{N}$ & $4^{\text {th }}$ & $e_{0}$ & $e_{1}$ & $e_{2}$ & $e_{3}$ & $e_{4}$ & $e_{5}$ \\
\hline \hline \multirow{4}{*}{6} & $c_{0}$ & -1 & & & & & \\
\cline { 2 - 8 } & $c_{1}$ & 18 & -10 & -0.5556 & -0.3395 & -0.2182 & -0.1515 \\
\cline { 2 - 8 } & $c_{2}$ & -48 & 40 & -3.3333 & 0.3395 & 0.3151 & 0.2484 \\
\cline { 2 - 8 } & $c_{3}$ & 32 & -32 & 4.8889 & 0 & -0.0969 & -0.0969 \\
\hline \multirow{4}{*}{7} & $c_{1}$ & -7 & 2 & 0.4184 & 0.1925 & 0.1139 & 0.0764 \\
\cline { 2 - 8 } & $c_{2}$ & 56 & -36 & -0.4388 & -0.9626 & -0.6568 & -0.4701 \\
\cline { 2 - 8 } & $c_{3}$ & -112 & 96 & -8.2857 & 0.7701 & 0.7535 & 0.6043 \\
\cline { 2 - 8 } 8 & $c_{4}$ & 64 & -64 & 9.3061 & 0 & -0.2105 & -0.2106 \\
\hline \multirow{4}{*}{8} & $c_{0}$ & 1 & & & & & \\
\cline { 2 - 8 } & $c_{1}$ & -32 & 14 & 1.625 & 0.8281 & 0.5112 & 0.352 \\
\cline { 2 - 8 } & $c_{2}$ & 160 & -112 & 1.375 & -2.4844 & -1.7788 & -1.3014 \\
\cline { 2 - 8 } & $c_{3}$ & -256 & 224 & -20 & 1.6563 & 1.707 & 1.389 \\
\cline { 2 - 8 } & $c_{4}$ & 128 & -128 & 18 & 0 & -0.4394 & -0.4397 \\
\hline
\end{tabular}

$t_{21}$

$$
A_{n}(2,1)=a_{n}, \quad n: 0 \sim N
$$

With the constraint of the impedance transformation

$$
z_{N+1}=r z_{0}
$$

and given the desired bandwidth ratio $B_{r}$, these $N+2$ equations can be used to solve for $\varepsilon_{m}$, and $z_{1}, z_{2}, z_{3} \ldots$ up to $z_{N+1}$. Generally, obtaining these $N+2$ equations involves a great deal of work regrading matrix operation and coefficient extraction. However, using available mathematical software packages (such as Mathematica, Matlab or Maple), these equations can be easily extracted through symbolic operations. Then these equations can be solved numerically using the software's built-in numerical functions.

\subsubsection{Comparison of Higher Order Marchand Baluns}

The feasibility of a wideband balun in a specific fabrication process is for the most part determined by the coupled-line structure. Two impedances are associated with this structure, the impedance between the coupled lines and the impedance of the outer shield with respect to the ground. They are $Z_{1}$ and $Z_{2}$ in the $1^{\text {st }}$ order balun, $Z_{2}$ and $Z_{3}$ in the $2^{\text {nd }}$ 


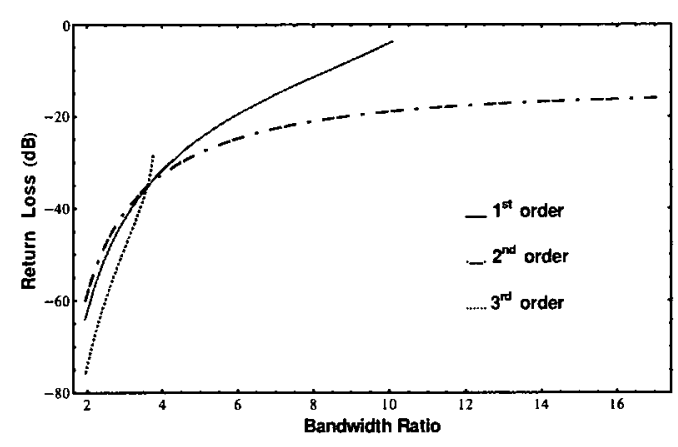

(a) Comparison of reflection coefficient.

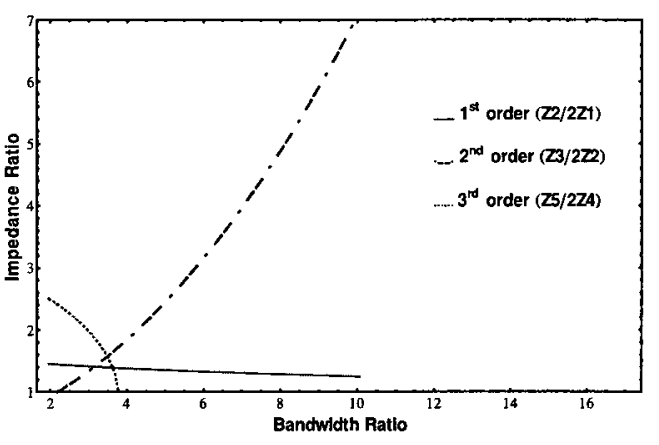

(b) Comparison of impedance ratios.

Figure 5.7: Comparison of 5-element baluns with $1^{\text {st }}, 2^{\text {nd }}$, and $3^{\text {rd }}$ order prewarping.

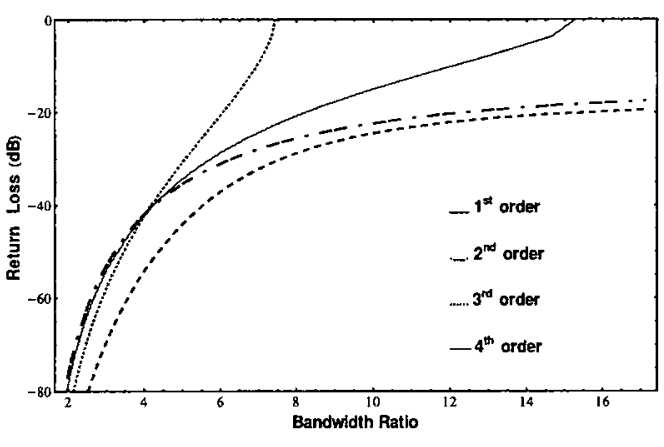

(a) Comparison of reflection coefficient.

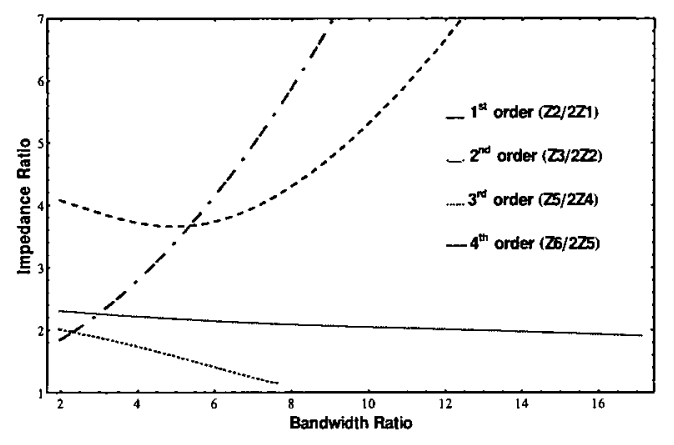

(b) Comparison of impedance ratios.

Figure 5.8: Comparison of 6-element baluns with $1^{\text {st }}, 2^{\text {nd }}, 3^{\text {rd }}$, and $4^{\text {th }}$ order prewarping.

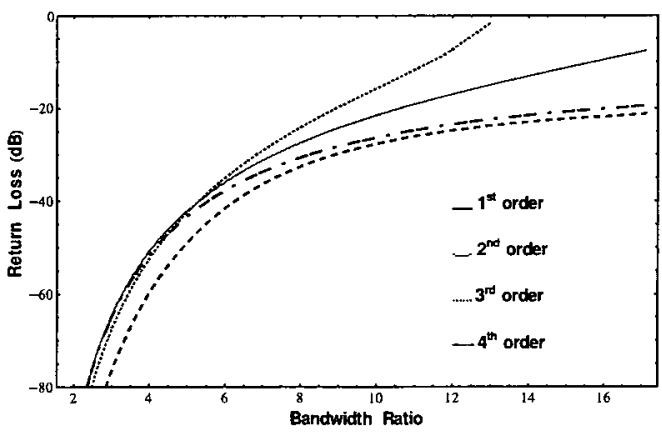

(a) Comparison of reflection coefficient.

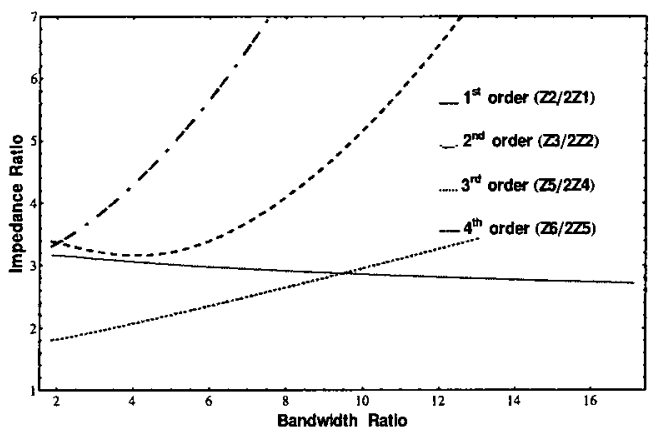

(b) Comparison of impedance ratios.

Figure 5.9: Comparison of 7-element baluns with $1^{\text {st }}, 2^{\text {nd }}, 3^{\text {rd }}$, and $4^{\text {th }}$ order prewarping. 
order balun, $Z_{4}$ and $Z_{5}$ in the $3^{r d}$ order balun, and finally $Z_{5}$ and $Z_{6}$ in the $4^{\text {th }}$ order balun as shown in Figure 5.5.

In a coaxial coupled-line structure or its equivalent planar version [68], the outer conductor needs to somehow enclose the inner conductor in order to achieve tight coupling. This physical structure makes it difficult to achieve a very low impedance between the coupled lines while maintaining a very high impedance of the outer shield with respect to ground. Similar difficulties can be found when using symmetrical coupled microstrip lines, where high even-mode impedance and low odd-mode impedance are required [69].

Based on the described design approach and the generalized topology of Marchand baluns described in previous sections, the $1^{\text {st }}-4^{\text {th }}$ order Marchand baluns using $5-7$ quarter-wave transmission-line elements are studied. An impedance transformation ratio of 2 is assumed, as is the usual case where all three ports are matched to the same impedance. Impedance ratios of the two coupled-line impedances are used to compare the difficulty of implementation among different balun configurations.

Figure 5.7 shows the comparison of the reflection coefficient and the impedance ratios for the $1^{\text {st }}, 2^{\text {nd }}$, and $3^{\text {rd }}$ order Marchand balun using 5 quarter-wave elements. It can be seen that, the maximum bandwidth ratio for a $3^{\text {rd }}$ order balun is approximately 3.5 having the best return loss performance. For a larger bandwidth ratio, the $2^{\text {nd }}$ order balun has the best return loss. However, the impedance ratio associated with the open-series coupledline structure goes up rapidly to 7 for a bandwidth ratio of 10 . The $1^{\text {st }}$ order balun would be the optimal configuration for a bandwidth ratio of $3.5 \sim 6$ as it achieves a reflection coefficient of $-20 \mathrm{~dB}$ with a relatively low impedance ratio.

Figure 5.8 shows the comparison of the reflection coefficient and the impedance ratios for the $1^{\text {st }}, 2^{\text {nd }}, 3^{\text {rd }}$, and $4^{\text {th }}$ order Marchand balun using 6 quarter-wave elements. It can be seen that, for a maximum reflection coefficient of $-20 \mathrm{~dB}$, the $3^{\text {rd }}$ order balun would be the optimal configuration for a bandwidth ratio of $2 \sim 6$. The $1^{\text {st }}$ order balun would be the optimal configuration for a bandwidth ratio of $6 \sim 8.5$. The $4^{\text {th }}$ order balun has the best return loss performance. However, the impedance ratio is generally large and it would be difficult to implement with an ordinary fabrication process. The $2^{\text {nd }}$ order balun would 


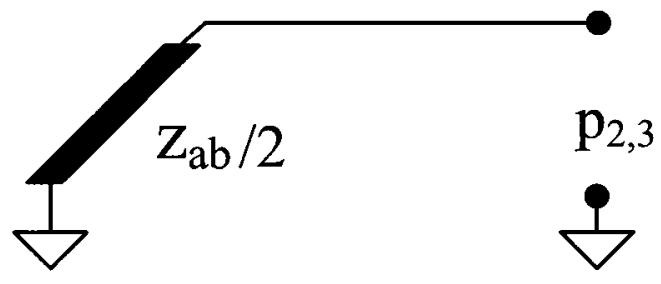

Figure 5.10: Marchand balun bisected equivalent circuit for even-mode excitations.

be suitable and practical for a bandwidth ratio less than 2 without resorting to a specific fabrication process.

Figure 5.9 shows the comparison of the reflection coefficient and the impedance ratios for the $1^{\text {st }}, 2^{\text {nd }}, 3^{\text {rd }}$, and $4^{\text {th }}$ order Marchand balun using 7 quarter-wave elements. From Figures 5.7-5.9, it can be observed that, with more elements used, the performance of the odd order baluns (with uncompensated structure as the first stage) approaches that of the even order baluns (with compensated structure as the first stage); however, the impedance ratio also increases.

In general, for a large bandwidth ratio, topologies using the compensated structure as the first stage achieve better return loss performance. However, a specific fabrication process is required for the large impedance ratio, such as in [70] where a customized coaxial structure is used for an impedance ratio of $13: 1$, and in [71] where polyimide layers stacked on GaAs wafer is used for an impedance ratio of $6: 1$. On the other hand, topologies using the uncompensated structure as the first stage have degraded performance. However, they are more feasible to implement using an ordinary fabrication process.

\subsection{Fully Matched Wideband Marchand Balun}

One key feature of Marchand balun structures is the introduction of an extra short-ended transmission-line section which enables the circuit to be symmetrical while looking in from the balanced port. This symmetry allows its equivalent circuit to be simplified for evenand odd-mode operations, and is shown in Figure 5.10 and Figure 5.2 respectively. For even-mode excitation, the simplified circuit is a short stub. Therefore, the even-mode 


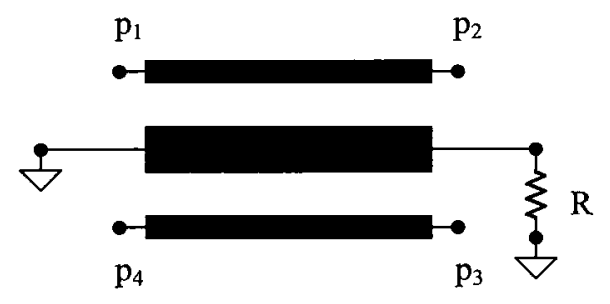

(a)

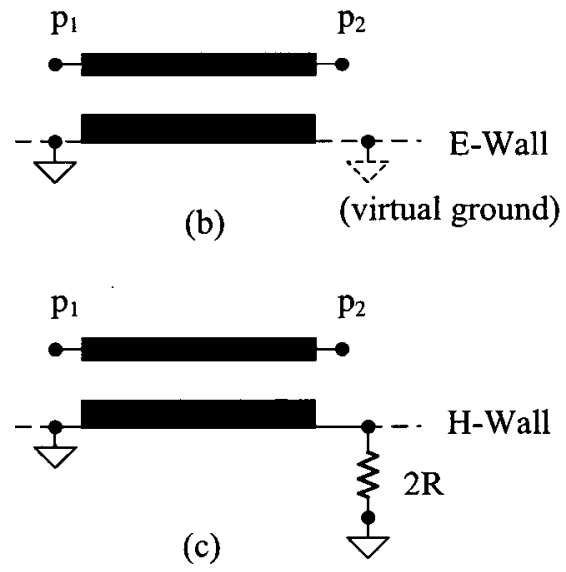

Figure 5.11: (a) Proposed coupled-line configuration for even-mode matching and its bisected equivalent circuit for (b) odd-mode and (c) even-mode excitations.

signal that is fed into the balanced ports will be reflected back. The signal fed into one of the two ports can be decomposed into even-mode and odd-mode portions, hence the even-mode portion leaks into the secondary port due to this reflection. This leakage causes poor isolation between the balanced ports of the Marchand balun.

In differential circuits, even-mode signal can be generated due to harmonic distortion, common mode interference and device mismatch. If the even-mode signal is not properly terminated, instability can be a problem, for high power amplifiers for instance, where large devices are usually used [72]. Inter-symbol-interference can occur for high data-rate and wideband applications due to the signal propagation between the balanced ports. Propagating even-mode signal also limits an active circuit's dynamic range.

\subsubsection{Proposed Even Mode Matching}

To solve the problem of isolation, multiple coupled-line sections will be inserted into the balanced line to provide even-mode matching. Each section consists of a coupled-threeline structure shown in Figure 5.11, where one terminal of the center line connects to ground and the other to a termination resistor. With odd-mode excitation, a virtual ground is formed along the center line, which effectively disables the termination resistor. 


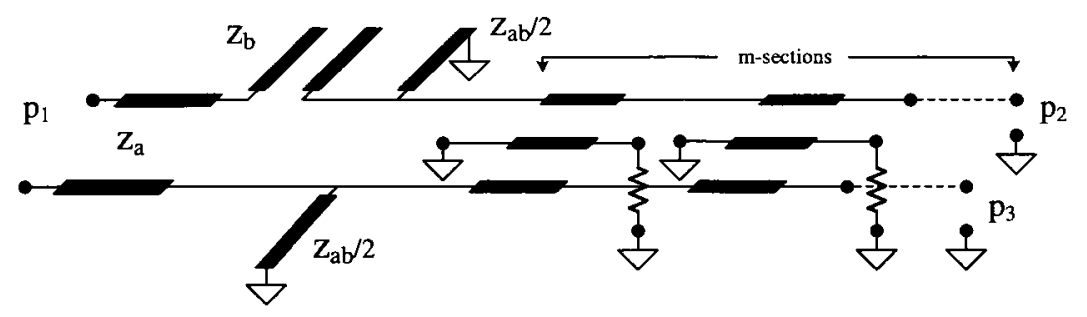

(a)

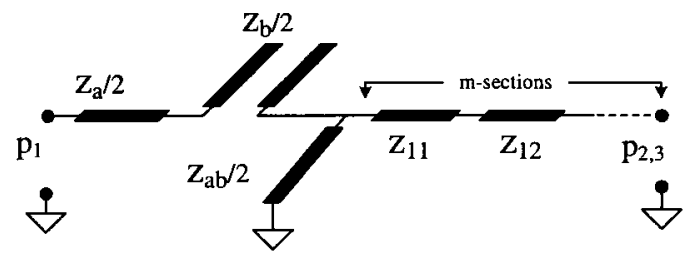

(b)

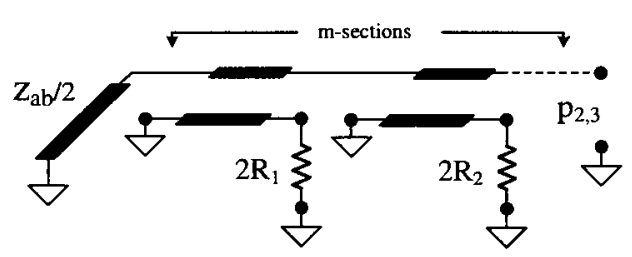

(c)

Figure 5.12: (a) Proposed fully matched Marchand balun and its bisected equivalent circuit for (b) odd-mode and (c) even-mode excitations.

With even-mode excitation, a magnetic wall is formed along the center line, which enables the termination resistor. By properly choosing the number of sections and the design parameters, adequate matching for even-mode excitation can be achieved over a wide bandwidth. Figure 5.12a shows the general circuit of the fully matched Marchand balun. It consists of a conventional Marchand balun followed by $N$ sections of even-mode matching. Its bisected equivalent circuits for odd-mode and even-mode excitations are shown in Figure $5.12 \mathrm{~b}$ and c respectively.

The synthesis steps for the odd-mode equivalent circuit was described in Section 5.2 of this chapter. With the characteristic impedances of the odd-mode circuit determined, the remaining problem is to compute the parameters for the even-mode circuit. The evenmode circuit involves a coupled-line structure, whose network model for this structure is derived based on the parameters of a general coupled-line structure and is discussed in the following sections. 


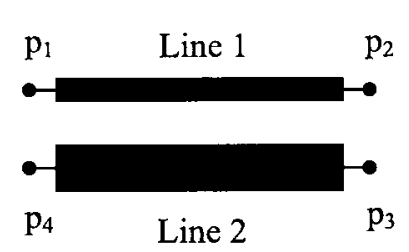

(a)

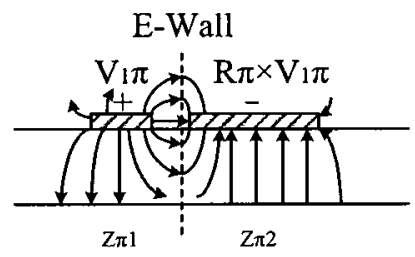

(b)

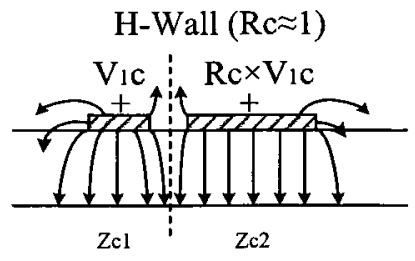

(c)

Figure 5.13: (a) A general uniformly coupled line (b) $\pi$-mode and (c) c-mode propagation.

\subsubsection{Coupled Line Models}

The quasi-TEM model of a general uniformly coupled-line structure (Figure 5.13a) can be represented by the lumped equivalent circuit of the coupled-line's differential length $[1,73]$ and shown in Figure 5.14. $V_{i}(z)$ and $I_{i}(z)$ denote the voltage and current on line $i$ as a function of distance $z$ along the transmission line. $L_{i}$ and $C_{i}$ denote the per-unit selfinductance and-capacitance of line $i . L_{m}$ and $C_{m}$ denote the per-unit mutual inductance and mutual capacitance between the lines. In frequency domain, the differential equations describing the coupled-line equivalent circuit are given as

$$
\begin{aligned}
& \frac{\partial V_{1}(z)}{\partial z}+j \omega L_{1} I_{1}(z)+j \omega L_{m} I_{2}(z)=0 \\
& \frac{\partial I_{1}(z)}{\partial z}+j \omega C_{1} V_{1}(z)-j \omega C_{m} V_{2}(z)=0 \\
& \frac{\partial V_{2}(z)}{\partial z}+j \omega L_{2} I_{2}(z)+j \omega L_{m} I_{1}(z)=0 \\
& \frac{\partial I_{2}(z)}{\partial z}+j \omega C_{2} V_{2}(z)-j \omega C_{m} V_{1}(z)=0
\end{aligned}
$$

By solving the differential equations, the characteristic parameters of the coupled-line can be obtained. The detailed results are listed in Appendix C. The coupled-line structure supports two normal modes: $\pi$-mode and $c$-mode [73]. These two modes propagate with phase velocities $v_{\pi}$ and $v_{c}$. Their associated line impedances are $Z_{\pi 1}, Z_{\pi 2}, Z_{c 1}$ and $Z_{c 2}$ (Fig.5.13 b and c). Along the coupled-line, the voltage ratios $\left(R_{c}\right.$ and $\left.R_{\pi}\right)$ of the two lines stay constant for each mode. The Z-matrix for the general coupled-line section in terms of normal-mode parameters has been summarized in [73] and [74] and is shown in Table 5.6, where $\phi_{c}$ and $\phi_{\pi}$ are the electrical lengths for the two normal modes of the coupled-line. 


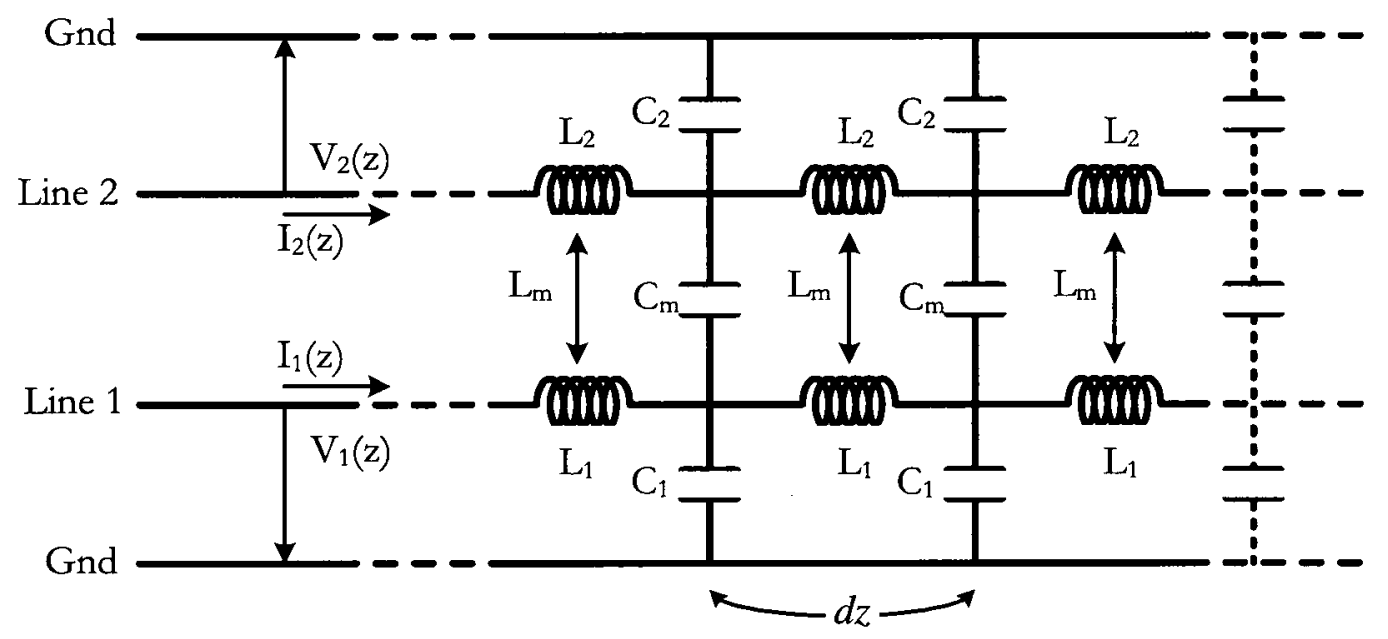

Figure 5.14: Lumped equivalent circuit of coupled asymmetrical transmission lines of differential length $d z[1]$.

Table 5.6: Z-matrix for a general coupled line section.

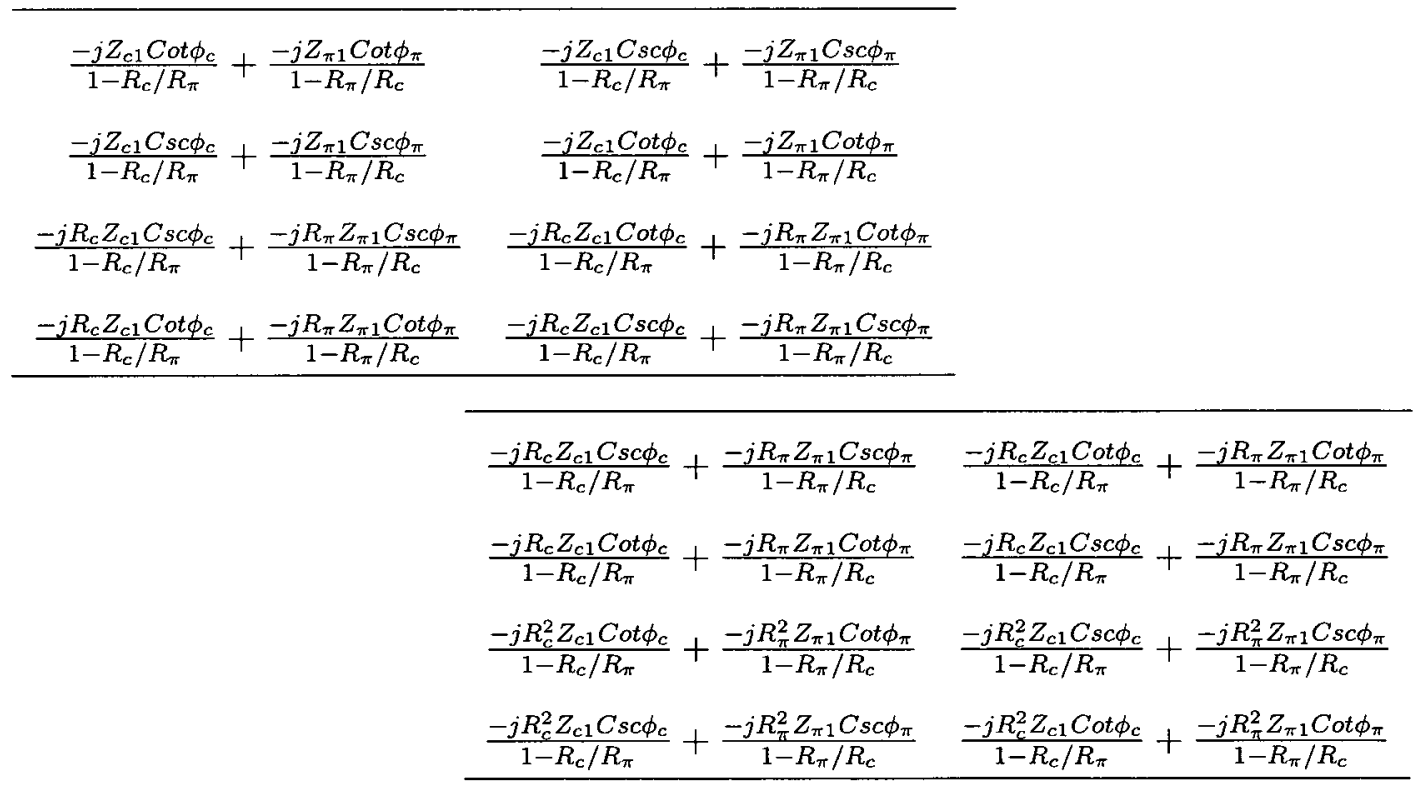


Table 5.7: Simplified Z-matrix for uniplanar case (assume $\phi_{c} \approx \phi_{\pi} \approx \phi$ ).

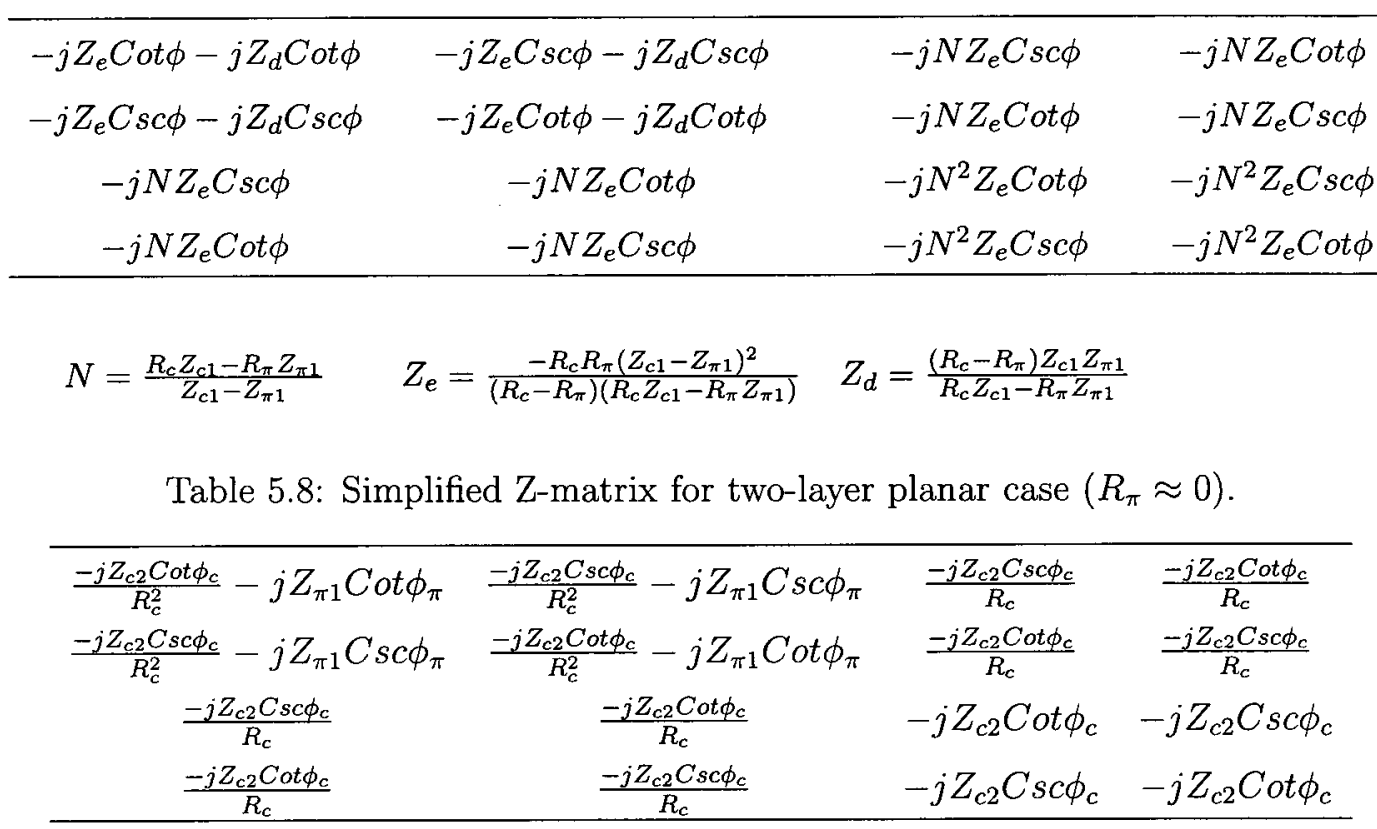

There are two ways to realize the coupled-line configuration of Figure 5.11c on a twoside PCB: 1) the coupled-lines reside on the same plane as shown in Figure $5.15 \mathrm{a} ; 2$ ) the coupled-lines reside on different layers as shown in Figure 5.15 b. For case 1, to simplify the model for circuit analysis, $\phi_{c} \approx \phi_{\pi} \approx \phi$ is assumed and the simplified Z-matrix is shown in Table 5.7. For case 2, the electrical field of line 1 is primarily confined between lines 1 and 2 , therefore $R_{\pi} \approx 0$. Its simplified Z-matrix is shown in Table 5.8. Following a similar procedure as described in [74], the network models can be obtained and are shown in Figure 5.15 (c) and (d) for case 1 (uniplanar) and case 2 (two-layer planar) respectively.

For even-mode operation, port 4 connects to ground and port 3 connects to the termination resistor. These network models can be further simplified to a two-port network as shown in Figure 5.16, where

Case 1: $Z_{1}=Z_{d}, Z_{2}=Z_{e}, n=N$

Case $2: Z_{1}=Z_{\pi 1}, Z_{2}=Z_{c 2}, n=R_{c}=1$. 


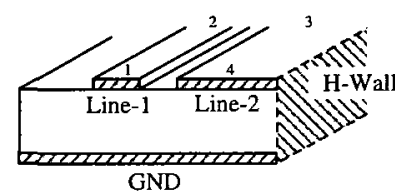

(a)

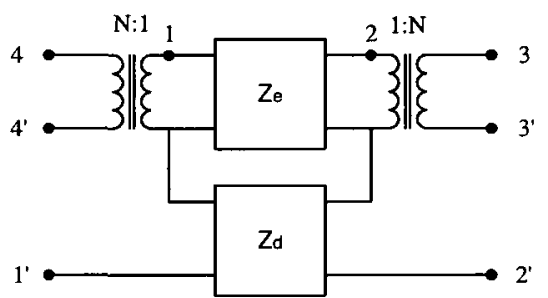

(c)

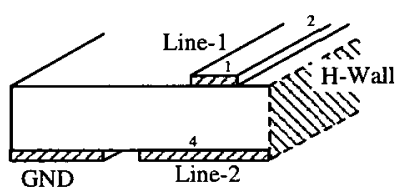

(b)

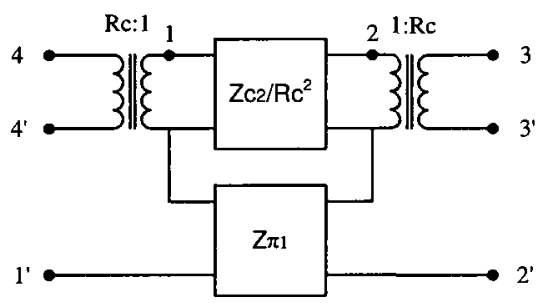

(d)

Figure 5.15: Coupled-line structures on a two-side substrate and their simplified models: (a) and (c) for uniplanar case; (b) and (d) for two-layer planar case.

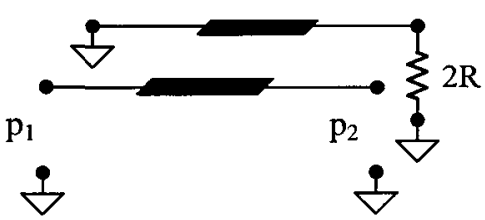

(a)

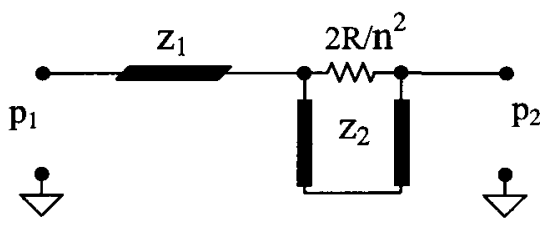

(b)

Figure 5.16: Two-port model of the even-mode matching section for even-mode excitation. 


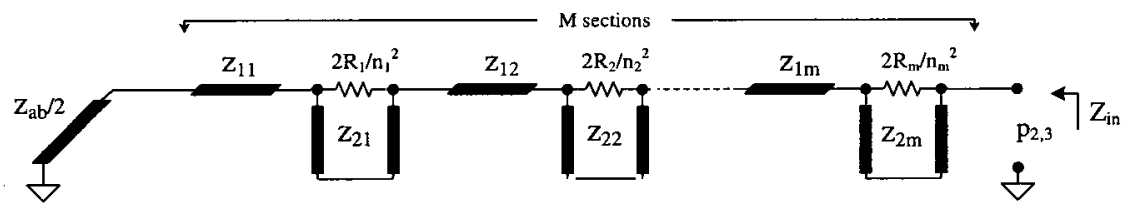

Figure 5.17: Even-mode network model of a m-section balun.

$Z_{1}$ is primarily determined by the odd-mode operation. Due to the existence of a magneticwall in even-mode operation and an electric-wall in odd-mode operation, $Z_{1}$ will be larger than the corresponding impedance of the odd-mode equivalent circuit. By applying the two-port model to the equivalent circuit in Figure $5.12 \mathrm{c}$, the even-mode network model of a Marchand balun with M-section even-mode matching can be obtained and is shown in Figure 5.17.

\subsubsection{Design Formulas, One Section $(\mathrm{M}=1)$}

With one section, the input impedance $Z_{i n}$ and the reflection coefficient $\Gamma_{\text {even }}$ are determined as

$$
\begin{gathered}
Z_{i n}=Z_{11} \frac{\frac{1}{2} Z_{a b} S+Z_{11} S}{Z_{11}+\frac{1}{2} Z_{a b} S^{2}}+\frac{\frac{2 R_{1}}{n_{1}^{2}} Z_{21} S}{\frac{2 R_{1}}{n_{1}^{2}}+Z_{21} S} \\
\Gamma_{\text {even }}=\frac{Z_{L}-Z_{i n}}{Z_{L}+Z_{\text {in }}}
\end{gathered}
$$

where $S$ is the Richard's transformation

$$
S=j \tan \phi=j \tan \frac{\pi f}{2 f_{o}} .
$$

$\phi$ is the electrical length of the transmission-line elements, $f_{o}$ is the frequency for which the transmission-lines are a quarter wavelength. $\Gamma_{\text {even }}$ of equation (5.3.7) can be expressed as a fraction of polynomials in $S$

$$
\Gamma_{e v e n}=\frac{c_{3} S^{3}+c_{2} S^{2}+c_{1} S+c_{0}}{d_{3} S^{3}+d_{2} S^{2}+d_{1} S+d_{0}}
$$

where the coefficients $c_{n}$ and $d_{n}$ are determined by $Z_{11}, Z_{21}, Z_{a b}$ and $R_{1}$. As $c$ s and $d \mathrm{~s}$ are all real numbers, $\Gamma_{\text {even }}$ has three zeros, one real zero and one pair of conjugate zeros. It 
can be proven that coefficient $c_{1}$ and $c_{3}$ can not both be zero simultaneously. Therefore, $Z_{\text {in }}$ can perfectly match $Z_{L}$ for at most two frequency points $\phi_{1}$ and $-\phi_{1}$

$$
\begin{aligned}
\Gamma_{\text {even }} & =\frac{c_{3}\left(S+r_{0}\right)\left(S+j \tan \phi_{1}\right)\left(S-j \tan \phi_{1}\right)}{d_{3} S^{3}+d_{2} S^{2}+d_{1} S+d_{0}} \\
& =\frac{c_{3}\left(S^{3}+r_{0} S^{2}+\tan ^{2} \phi_{1} S+r_{0} \tan ^{2} \phi_{1}\right)}{d_{3} S^{3}+d_{2} S^{2}+d_{1} S+d_{0}} .
\end{aligned}
$$

Comparing the numerators of equation (5.3.9) and (5.3.11), we have

$$
\begin{aligned}
& c_{2}=c_{3} r_{0} \\
& c_{1}=c_{3} \tan ^{2} \phi_{1} \\
& c_{0}=c_{3} r_{0} \tan ^{2} \phi_{1} .
\end{aligned}
$$

By solving this set of equations, design parameters $R_{1}$ and $Z_{21}$ can be formulated as in (5.3.13) and (5.3.14) in terms of $Z_{11}$ and $Z_{a b}$

$$
\begin{aligned}
& R_{1}=\frac{\left(4 Z_{11}^{2} Z_{L}^{2}+\tan ^{2} \phi_{1} Z_{11}\left(4 Z_{11}^{3}+4 Z_{11}^{2} Z_{a b}+Z_{11} Z_{a b}^{2}-4 Z_{a b} Z_{L}^{2}\right)+\tan ^{4} \phi_{1} Z_{a b}^{2} Z_{L}^{2}\right) n^{2}}{2\left(2 Z_{11}-\tan ^{2} \phi_{1} Z_{a b}\right)^{2} Z_{L}} \\
& Z_{21}=\frac{\left(4 Z_{11}^{2} Z_{L}^{2}+\tan ^{2} \phi_{1} Z_{11}\left(4 Z_{11}^{3}+4 Z_{11}^{2} Z_{a b}+Z_{11} Z_{a b}^{2}-4 Z_{a b} Z_{L}^{2}\right)+\tan ^{4} \phi_{1} Z_{a b}^{2} Z_{L}^{2}\right)}{-\tan ^{2} \phi_{1} Z_{11}\left(2 Z_{11}+Z_{a b}\right)\left(2 Z_{11}-\tan ^{2} \phi_{1} Z_{a b}\right)}
\end{aligned}
$$

By varying the frequency $\phi_{1}$, the equal-ripple bandwidth $(B W)$ can be adjusted (Figure 5.18). It can be concluded from equation (5.3.13) and (5.3.14) that

$$
\left(2 Z_{11}-\tan ^{2} \phi_{1} Z_{a b}\right)<0
$$

should be satisfied to guarantee $R_{1}$ and $Z_{21}$ are positive. This condition produces the lower bound of the $\phi_{1}$ frequency as

$$
\phi_{1}>\phi_{L}=\tan ^{-1} \sqrt{\frac{Z_{11}}{\frac{1}{2} Z_{a b}}} .
$$

This is due to the resonance of $Z_{11}$ with the shorted-line $Z_{a b}$ at $\phi_{L}$, which increases the input impedance dramatically. Hence, a small $\phi_{L}$ is desired for a large bandwidth. 


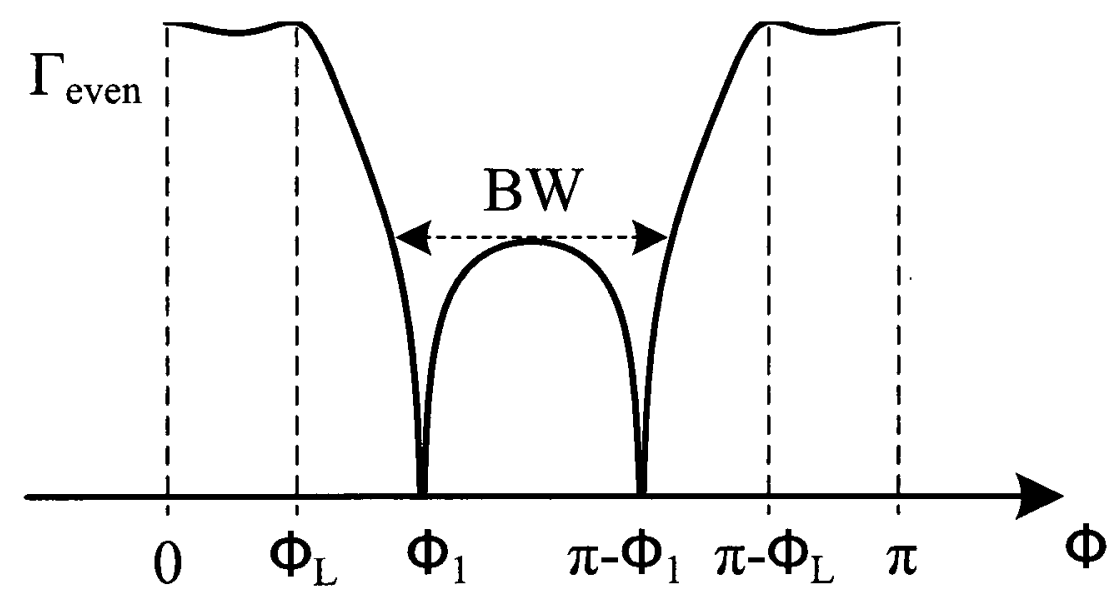

Figure 5.18: Common mode reflection coefficient.

\subsubsection{Design Procedure, Multiple Sections $(M \geq 2)$}

In order to decrease $\phi_{L}, Z_{11}$ should be made small when compared with $\frac{1}{2} Z_{a b}$ as can be seen in (5.3.16). Concluded from odd-mode analysis, it is desirable to decrease the equivalent impedance while looking towards the balanced load. Therefore, it offers benefits for both even- and odd-mode operations to incorporate multiple sections of the even-mode matching circuit to the existing balun structure.

The design procedure described in Section 5.3.3 can be generalized to the M-section circuit in Figure 5.17. First, by means of the fundamental transmission-line theory, the input impedance $Z_{\text {in }}$ can be determined. The reflection coefficient $\Gamma_{\text {even }}$ can be expressed as a fraction of the polynomials in $S$ of degree $2 M+1$

$$
\Gamma_{\text {even }}=\frac{\sum_{m=0}^{2 M+1} c_{m} S^{m}}{\sum_{m=0}^{2 M+1} d_{m} S^{m}} .
$$

The coefficients $c_{m}$ and $d_{m}$ are determined by $Z_{1 m}, Z_{2 m}, Z_{a b}$ and $R_{m} . Z_{1 m}$ and $Z_{a b}$ are parameters primarily determined by the odd-mode operation. $Z_{2 m}$ and $R_{m}$ are the design parameters to be determined for even-mode operation. Assuming perfect match at $\pm \phi_{1}$, 
$\pm \phi_{2}, \ldots$ and $\pm \phi_{M}$, the reflection coefficient $\left(\Gamma_{\text {even }}\right)$ can be expressed as

$$
\Gamma_{\text {even }}=\frac{c_{2 M+1}\left(S+r_{0}\right) \prod_{m=1}^{M}\left(S^{2}+\tan ^{2} \phi_{m}\right)}{\sum_{m=0}^{2 M+1} d_{m} S^{m}} .
$$

Expanding the numerator of (5.3.18), then comparing it to the numerator of (5.3.17) and letting the corresponding coefficients equal, we obtain $2 M+1$ equations. Finally, by solving these equations, design parameters $Z_{2 m}$ and $R_{m}$ can be determined.

Deriving symbolic formulas for $Z_{2 m}$ and $R_{m}$ in terms of $\phi_{m}, Z_{1 m}$ and $Z_{a b}$ becomes increasingly more difficult for $M \geq 2$. However, given the exact values of $\phi_{m}, Z_{1 m}$ and $Z_{a b}$, parameters $Z_{2 m}$ and $R_{m}$ can be obtained by numerical techniques. With the many available mathematic software packages such as Mathematica, Maple or MatLab, this job can be easily accomplished on a regular PC. The zero-frequencies $\phi_{1}, \phi_{2}, \ldots$ and $\phi_{M}$ are chosen to produce an equal-ripple response. Given the operation bandwidth, optimization techniques can be applied to obtain these frequencies. This process can be completed manually by following a simple rule: moving two neighbor zeros closer in frequency to decrease the ripple between the two zeros or moving them further apart in order to increase the ripple between them. Several iterations can make the frequency response approximately equal-ripple.

\subsection{Measurement Results}

A compensated Marchand balun with 2-section even-mode matching has been designed for combining the differential output of the predistorter. This balun is intended to work at a center frequency of $4 \mathrm{GHz}$ and cover the frequency range of 2-6 GHz. The calculated characteristic impedances of this balun are listed in Table 5.9. For even-mode operation, the calculated impedance of $Z_{21}$ is $192 \Omega$, which would be too large to be practical in a real environment. Without changing the equivalent circuit, the topology of the even-mode circuit is modified as shown in Figure 5.19. In this way, the impedance is effectively reduced by half.

This balun is designed on a substrate with a relative dielectric constant of 3.38 and 
Table 5.9: Characteristic impedances of the designed balun

\begin{tabular}{c}
\begin{tabular}{c|c|c|c|c|c|c|c|}
\hline \hline \multirow{2}{*}{$\begin{array}{c}\text { odd- } \\
\text { mode }\end{array}$} & $Z_{0}$ & $Z_{a}$ & $Z_{b}$ & $Z_{a b} / 2$ & $Z_{11}$ & $Z_{12}$ & $Z_{L}$ \\
\cline { 2 - 8 } & $50 \Omega$ & $55.4 \Omega$ & $28.4 \Omega$ & $52.6 \Omega$ & $35.4 \Omega$ & $45.1 \Omega$ & $50 \Omega$ \\
\hline \hline \multirow{2}{*}{ even } & $Z_{a b} / 2$ & $Z_{11}$ & $Z_{12}$ & $Z_{21}$ & $Z_{22}$ & $2 R_{1}$ & $2 R_{2}$ \\
\cline { 2 - 8 } & $105 \Omega$ & $35.4 \Omega$ & $45.1 \Omega$ & $192 \Omega$ & $36.0 \Omega$ & $180 \Omega$ & $52.3 \Omega$ \\
\hline
\end{tabular} \\
\hline
\end{tabular}

Figure 5.19: Modified even-mode topology.

a thickness of 20 (mils). Figure 5.20 illustrates the layout of the designed balun. The board dimension is $36(\mathrm{~mm}) \times 70(\mathrm{~mm})$. Quarter-wave elements $Z_{21}$ and $Z_{22}$ are made by cutting slots on the bottom plane. Figure 5.21 is a zoomed-in view of one section of the layout, which shows the transition where the top metal runs over a narrow slot on the bottom plane. Due to the existence of this narrow slot, the bottom edges inside the circle become floating in stead of being connected as ground. The line impedance increases at the transition area. To compensate this effect, the top metal traces are made wider and closer to reduce the impedance and enhance the coupling between the top traces. These narrow slots also affect the impedances of the short-circuited $Z_{21}$ and $Z_{22}$. To compensate, the lengths of the slots beside the top metal trace were adjusted for matching the responses of ideal quarter-wave elements. The bottom metal traces for $Z_{21}$ and $Z_{22}$ are designed to be wider than the top traces. In this way, top traces are shielded from the ground and $R_{\pi}=0$ can be assumed, hence the effect of the slot width on $Z_{11}$ and $Z_{12}$ can be neglected.

The balun's 3D model was constructed gradually in Ansoft HFSS EM simulation environment. It started with only $Z_{a}, Z_{b}$ and $Z_{a b}$ sections modeled in HFSS. In order to obtain the S-parameters of the entire balun, the rest of the circuits were represented as 


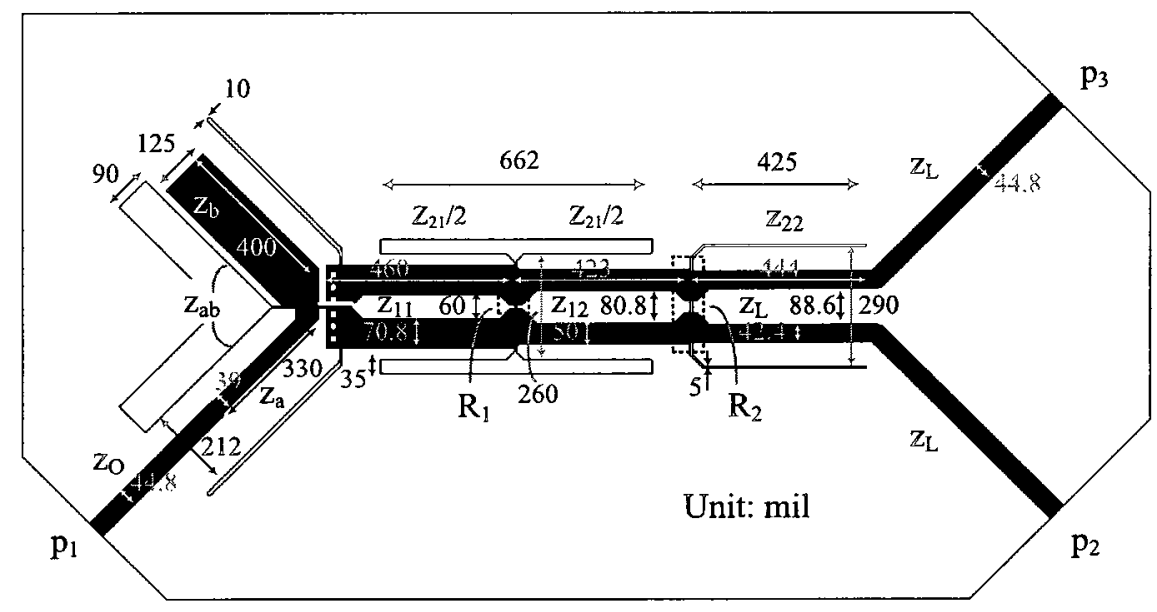

Figure 5.20: PCB layout of the desigmed balun.

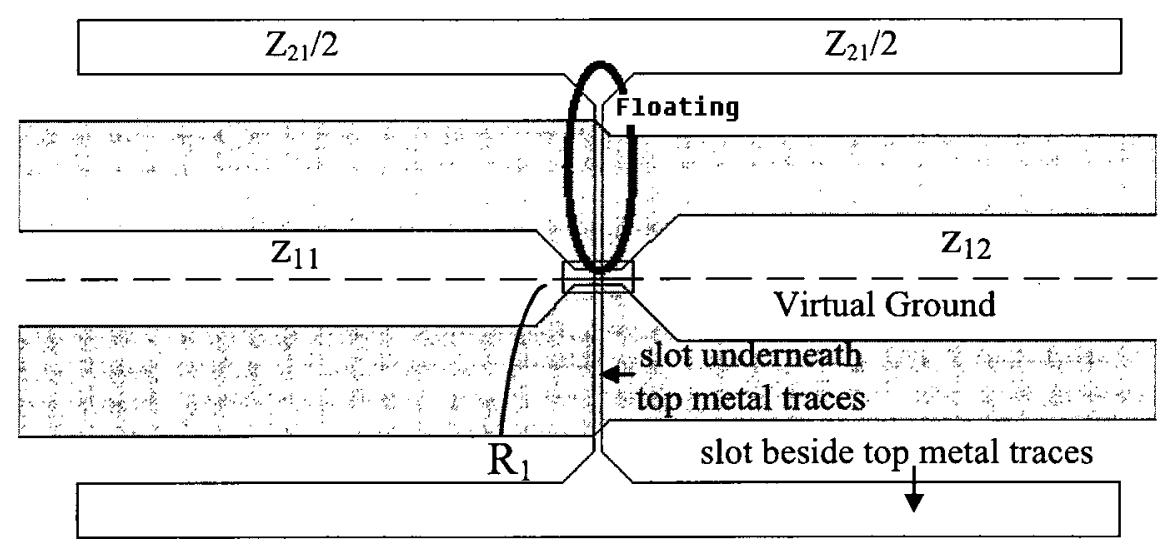

Figure 5.21: A zoomed-in view of the layout. 


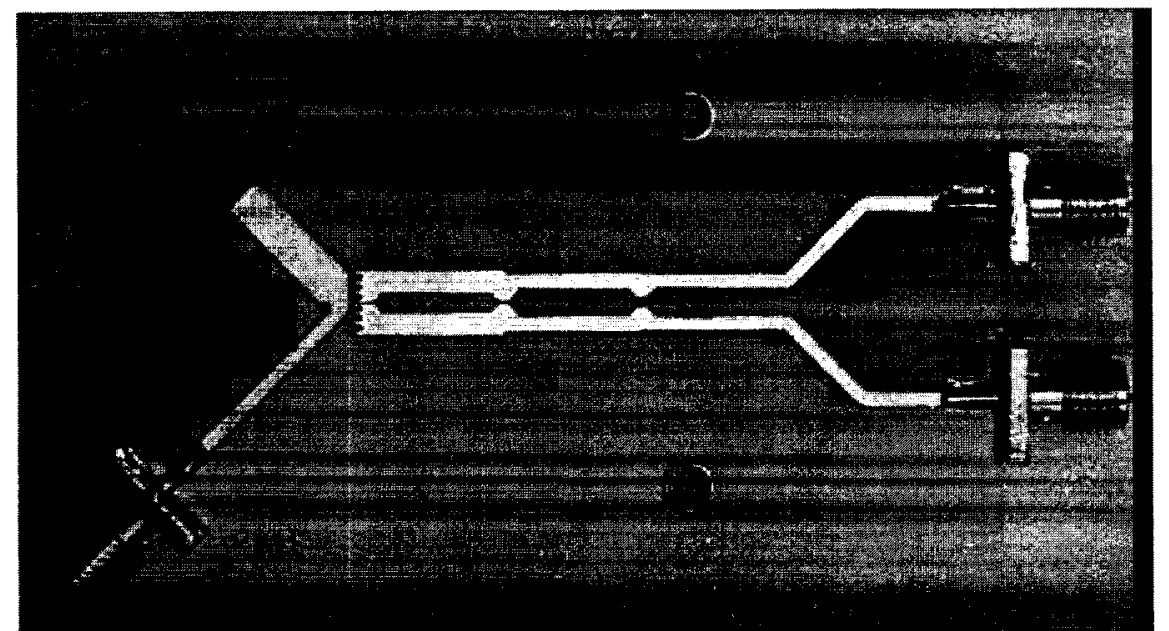

(a) Top

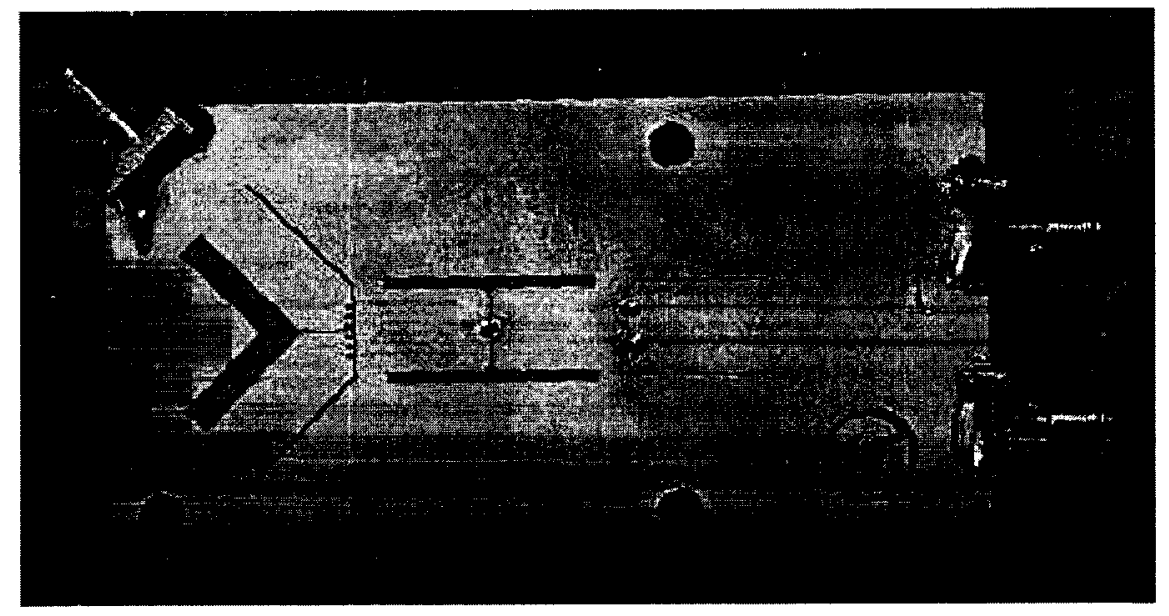

(b) Bottom

Figure 5.22: Photograph of the fabricated balun. 


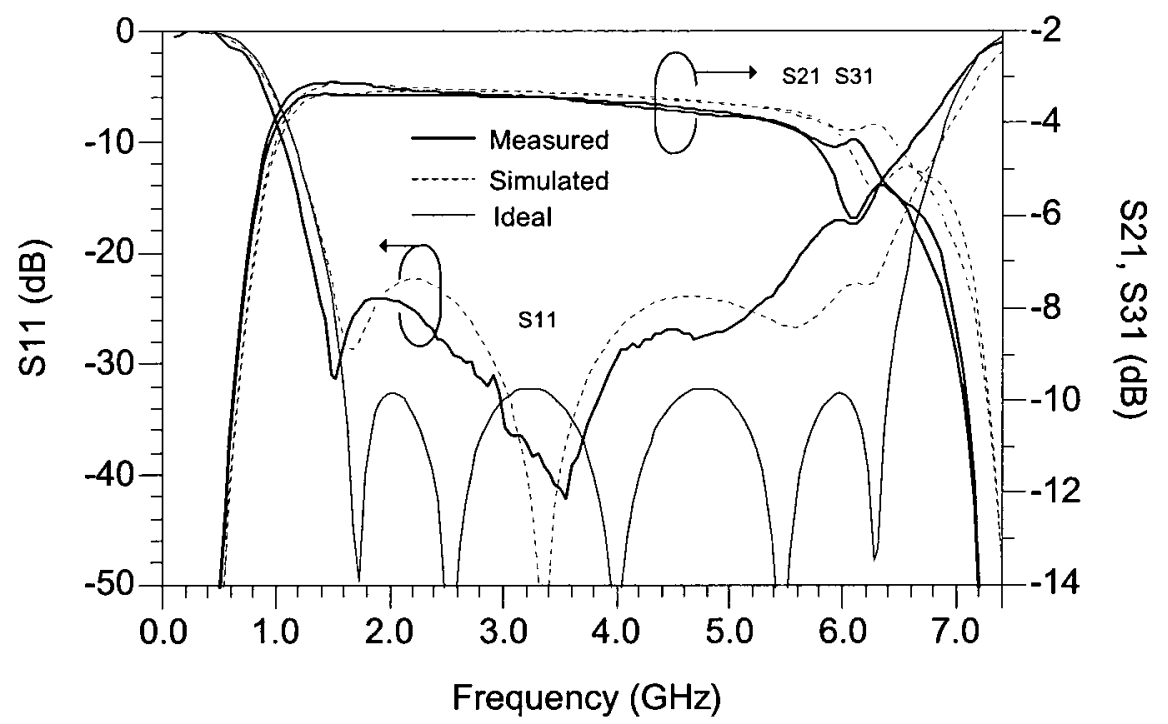

Figure 5.23: Measured $S_{11}, S_{21}$ and $S_{31}$ compared with EM simulated results.

ideal elements by a few equations. Then gradually add more sections in the model and remove the ideal ones in the equations. In this way, a fine tuning on physical dimensions of each section can be performed to explore their effects on the overall performance.

The simulated results of the balun modeled in HFSS and the reflection coefficients obtained by using ideal components are included in Figure 5.23 and 5.24. The difference between the two results is mainly due to the physical implementation of $Z_{21}$ and $Z_{22}$ whose frequency responses do not exactly match to those of ideal elements. Figure 5.22 shows the photograph of the fabricated balun. The balun is measured by Agilent's Vector Network Analyzer, two ports at a time, with the unused port terminated in a $50 \Omega$ load. Deembedding is then performed to remove the effects of the SMA connectors. The results obtained are shown in Figure 5.23 and 5.24. A good agreement can be observed between the measured and the simulated results. Compared with the theoretical results of baluns without even mode matching, the measured reflection coefficient $S_{22}$ and $S_{33}$ and isolation coefficient $S_{23}$ has improved from $-6 \mathrm{~dB}$ to better than $-15 \mathrm{~dB}$ for the frequency range from $1.5 \mathrm{GHz}$ to $6 \mathrm{GHz}$. Phase and amplitude imbalances between the balanced outputs are 


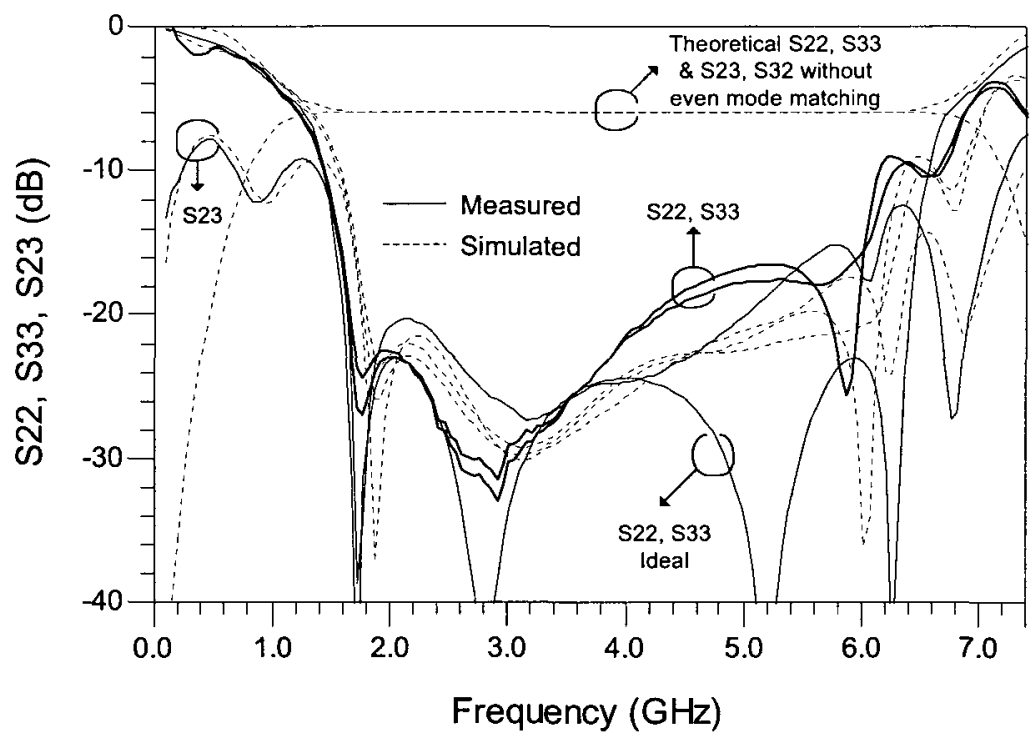

Figure 5.24: Measured $S_{22}, S_{33}$ and $S_{23}$ compared with EM simulated results.

also important properties to characterize the performance of a balun, and are defined as

$$
\begin{aligned}
& \text { phase imbalance : } \quad\left|\angle S_{21}-\angle S_{31}\right|-180^{\circ} \\
& \text { amplitude imbalance : } 20 \log \frac{\left|S_{21}\right|}{\left|S_{31}\right|}
\end{aligned}
$$

Better than $2^{\circ}$ phase imbalance can be observed from dc to $5.5 \mathrm{GHz}$ and better than 0.3 $\mathrm{dB}$ amplitude imbalance can be observed from dc to $5.8 \mathrm{GHz}$ as shown in Figure 5.25.

\subsection{Summary}

This chapter describes the design technique that is used for designing a wideband fully matched Marchand balun. A balun has been designed and the measured results demonstrates a satisfactory performance in the frequency range of 2-6 GHz. This operation bandwidth should be enough to cover the HD2 and IM3 band of the predistorter output. 


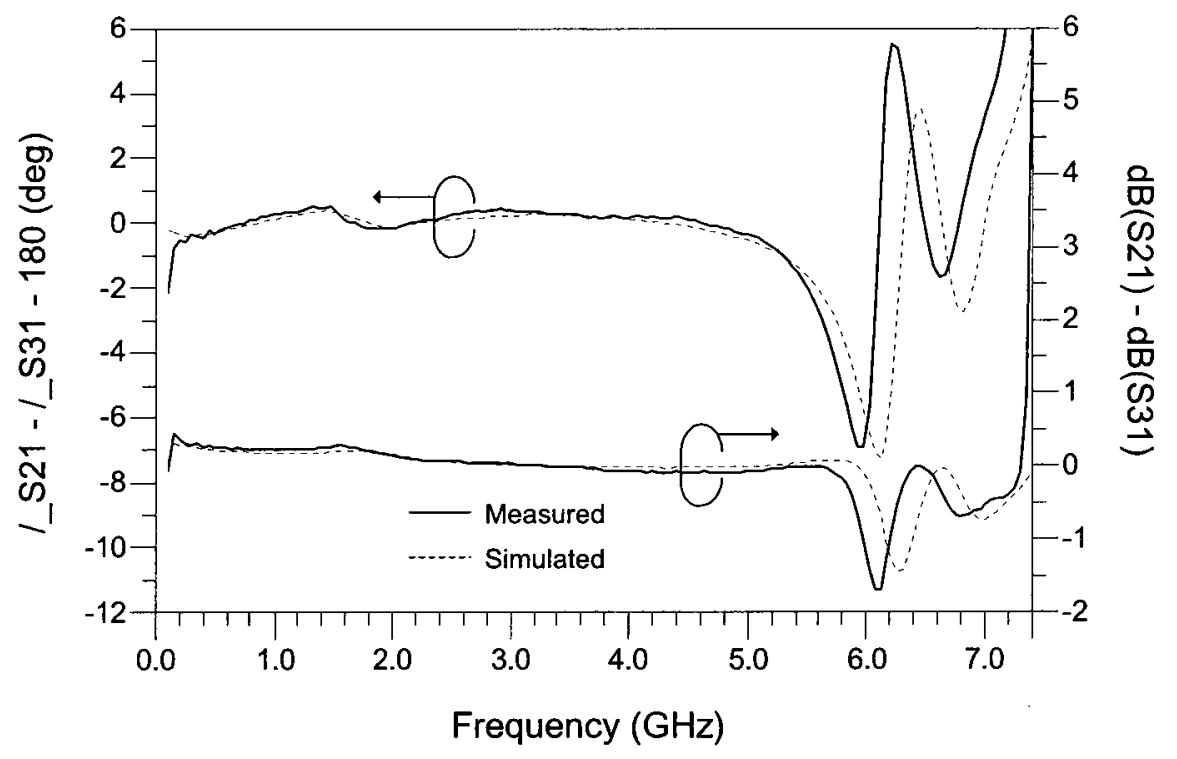

Figure 5.25: Measured phase and amplitude imbalance compared with EM simulated results. 


\section{Chapter 6}

\section{Adaptive Calibration and Measurement Results}

The semiconductor laser exhibits fluctuations in characteristics from component to component and changes in performance due to temperature change and aging effect. It is necessary to calibrate the predistortion circuit adaptively in order to counteract the distortion behavior of an individual laser diode and correct the drift errors that would occur over time and temperature.

This chapter presents in detail the calibration algorithm that is used to adjust the tuning coefficients of the implemented predistorter. This algorithm is a direct feedback algorithm, not an optimization algorithm, which suggests a simple solution for analog implementation.

\subsection{General Considerations}

The calibration is feasible in a discrete time system. A problem of using a continuous time analog system is that the analog value can not be held for a long time in integrated circuits due to current leakage and substrate noise. There are techniques to address this problem, but they add complexity. The calibration process of the predistorter can be described as follows: first, pilot tones are applied to the predistorter input; the laser outputs are detected by a photodiode; harmonic components and intermodulation components are extracted for estimating the distortion level; then the tuning voltages are adjusted according to an 
algorithm to reduce the distortion level. After calibration is done, the tuning voltages of the predistorter are maintained stable for transmitting information-bearing signals. This requires the tuning voltages to be stored in a digital form and a digital-analog converter to provide the tuning voltage.

The calibration problem in this proposed work can be described as either a case of optimization or a case of equations-solving, since the purpose of the calibration is to minimize the distortion (optimization problem) or match the frequency profile (equations solving problem). The following sections will discuss the potential solutions for both cases.

\subsubsection{Optimization}

Optimization to minimize distortion is to solve the problem of

$$
\frac{\partial D}{\partial a_{i}}=0 \quad\left(D>0, D^{\prime \prime}>0\right) .
$$

$D$ in the equation 6.1.1 is the cost function, which is, for example, the weighted sum of harmonic distortion and intermodulation distortion powers and $a_{i}$ is the tuning coefficient for each of the $i$ tank circuits. The benefit of optimization is that it only requires to detect the distortion power, which greatly simplifies the detection circuit. However, the calibration algorithm has to be realized by a digital system because of the requirement for memories. As can be seen from (6.1.1), derivatives or the equivalent need to be estimated for searching for the optimum. Memories are required to store the detected values of the cost function for different tuning coefficients. Then the derivative (6.1.1) can be estimated by comparing the cost function values stored in the memory.

Optimization methods can be deterministic or heuristic. Known methods are gradient search, Newton method and its modifications, simplex algorithm, simulated annealing and genetic algorithms $[75,76]$. In the simulation environment, the steepest-gradient method was used. No problems of convergence were encountered in the test cases and the distortion cost function $D$ was minimized after the optimization procedure. 


\subsubsection{Iteration (Feedback)}

As discussed in Chapter 3, the tuning coefficients can be obtained by matching the response of the multi-tank circuit to the required frequency profile at a few frequency points. Therefore, the calibration becomes the problem of solving

$$
\vec{D}_{n}\left(a_{1}, a_{2}, \ldots, a_{i}\right)=0
$$

where $\vec{D}_{n}$ are the distortion signals in the vector form. An iteration method can be applied to solve this set of equations. For a quick convergence, Newton-Raphson iteration is usually used in numerical analysis to solve linear or nonlinear equations. The idea of the method is as follows: one starts with an initial guess which is reasonably close to the true solution, then the function is approximated by its tangent line (derivative), and one computes the $\mathrm{x}$-intercept of this tangent line to obtain the solution for the equations. By repeating this process a few times, the solution will be updated successively to approximately satisfy equations (6.1.2), as illustrated in Figure 6.1. However, this method has the same drawback of calculating the derivatives. The alternative is using the fix-point iteration, which is illustrated in Figure 6.2. In fixed-point iteration, there is no need to accurately calculate the slops (derivatives) of the curve. A coarse estimation of the derivatives can be applied and the same derivatives are used for the following iteration procedure. Compared with the Newton-Raphson iteration, fixed-point iteration takes more step to converge to a desired error level. However, the fixed-point iteration does not require the calculation of derivatives, which greatly simplifies the circuit design and makes it possible for an analog implementation.

\subsection{Adaptive Calibration using Multiple Variable Feed- back}

As discussed in the previous section, the iteration method uses direct feedback to find the solutions of the targeted equations, which provides the advantage of implementation in the analog circuit, thus eliminating the use of expensive DSP unit and memories. 


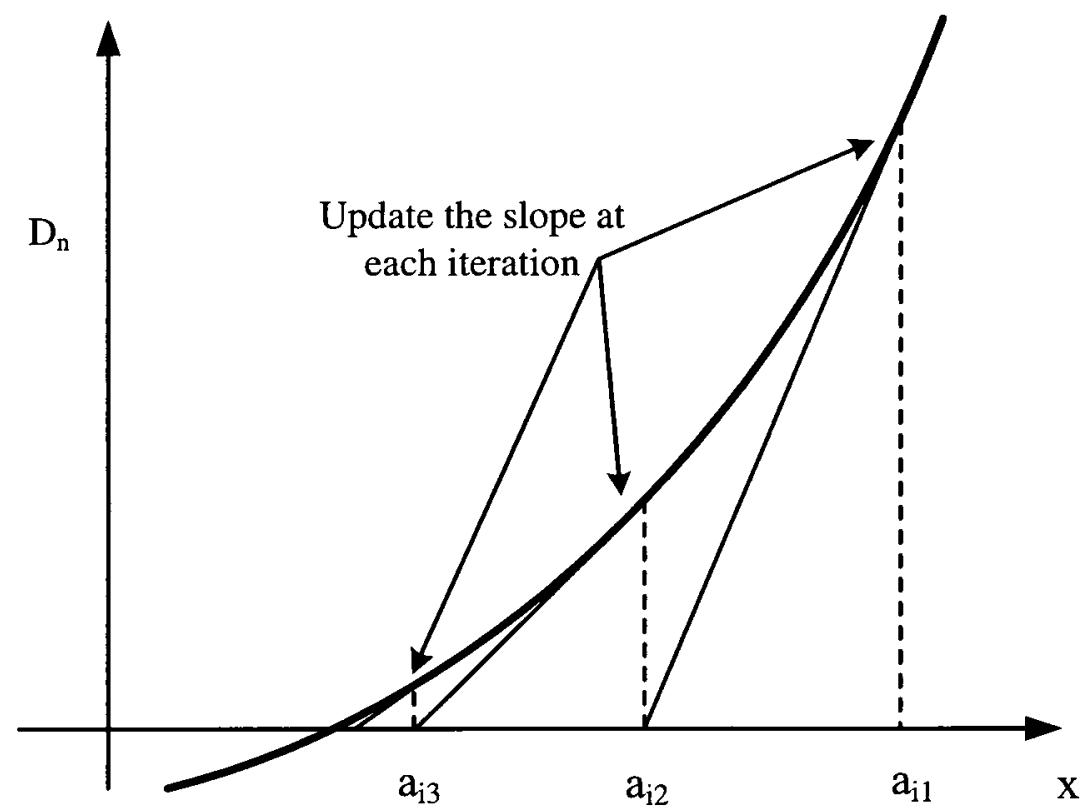

(a)

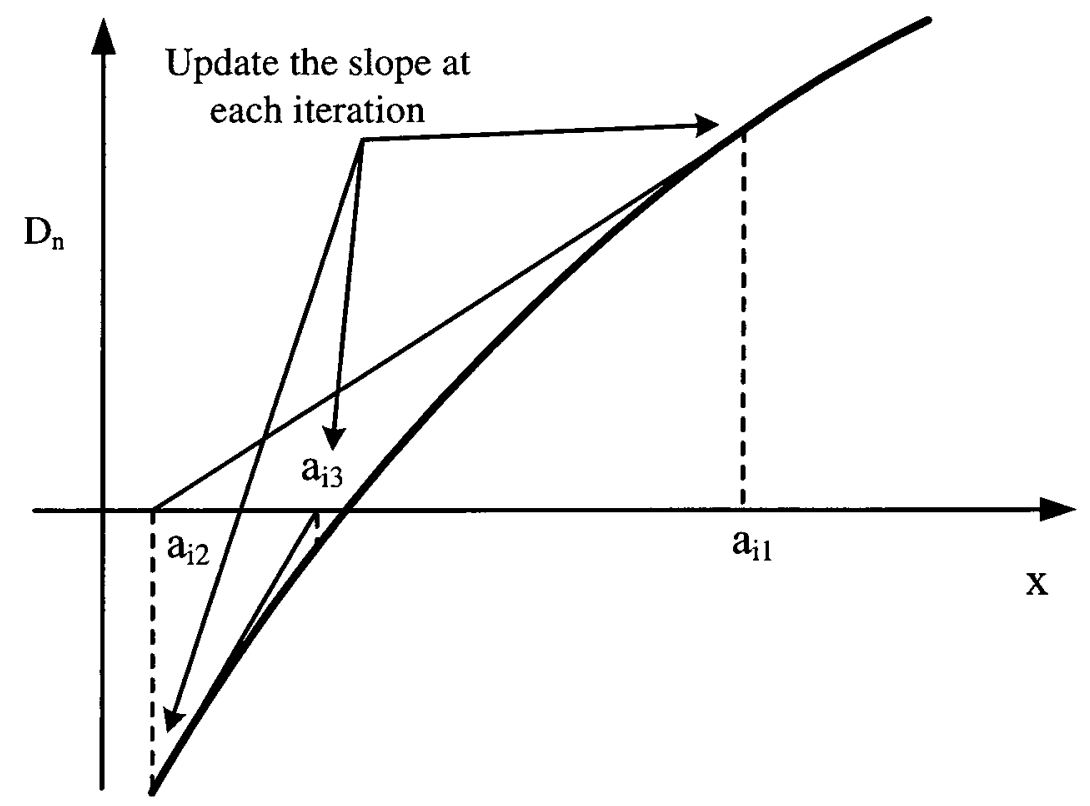

(b)

Figure 6.1: Newton-Raphson iteration for (a) expansive function and (b) compressive function. 


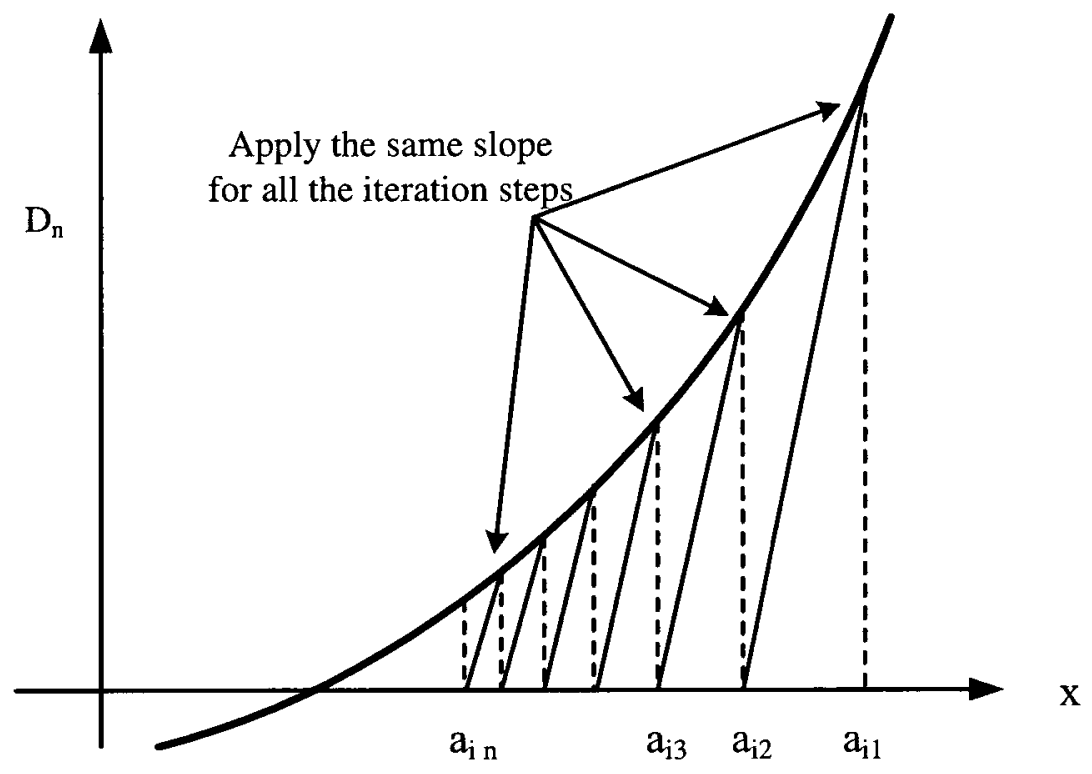

(a)

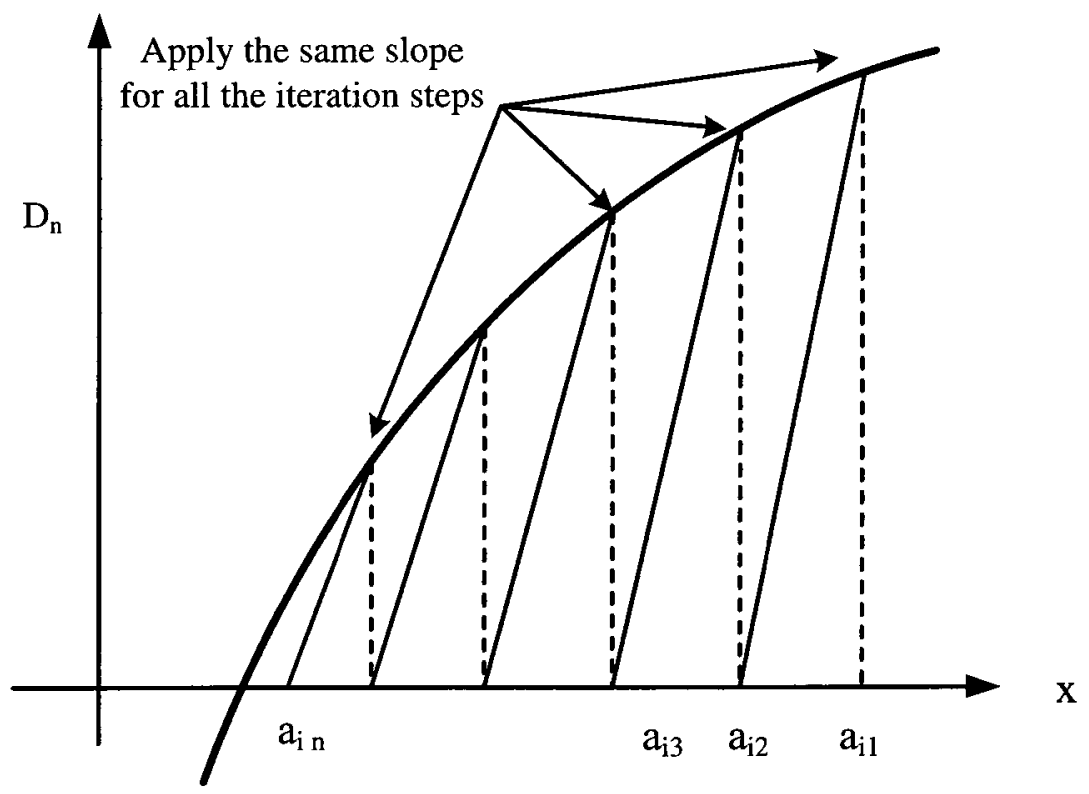

(b)

Figure 6.2: Fixed-point iteration for (a) expansive function and (b) compressive function. 


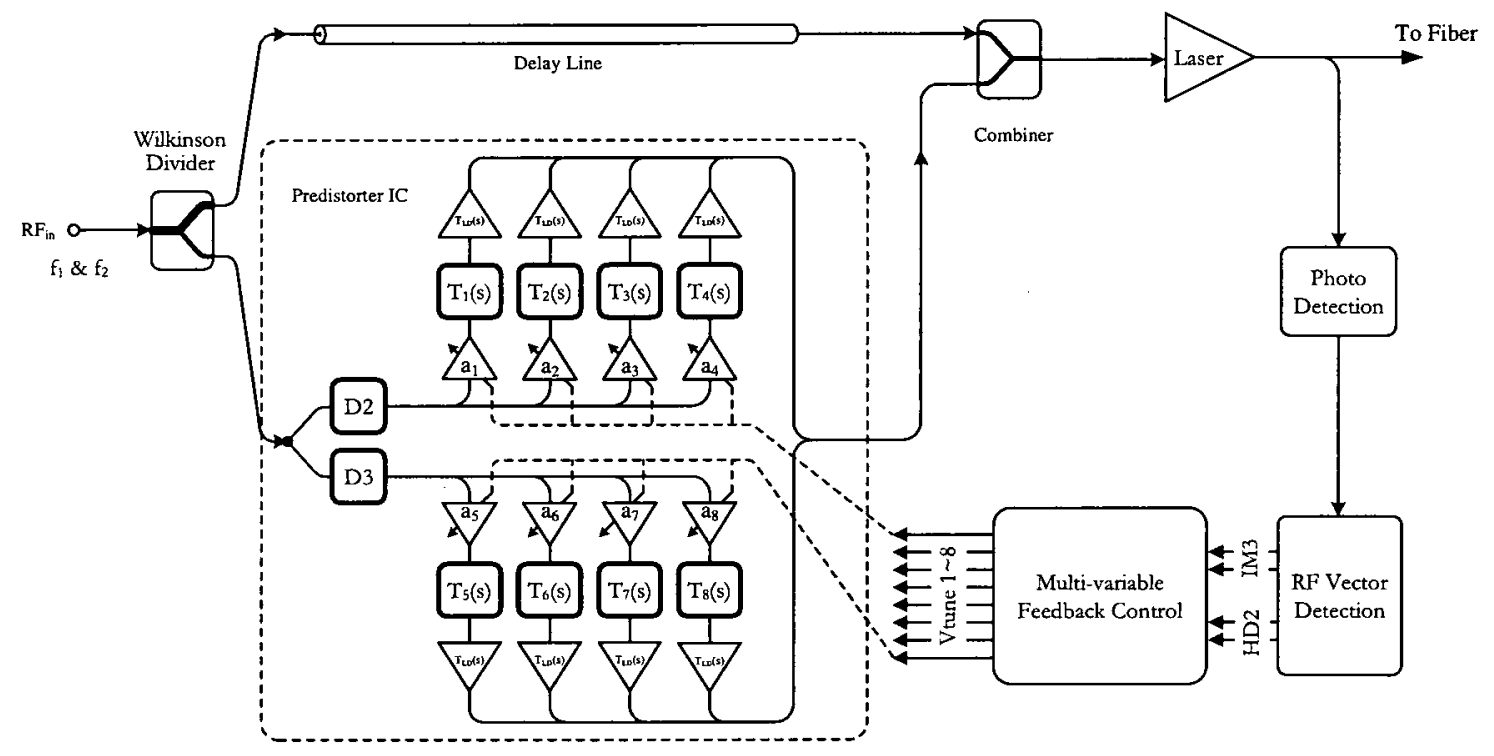

Figure 6.3: Block diagram of the predistorter IC and the feedback control.

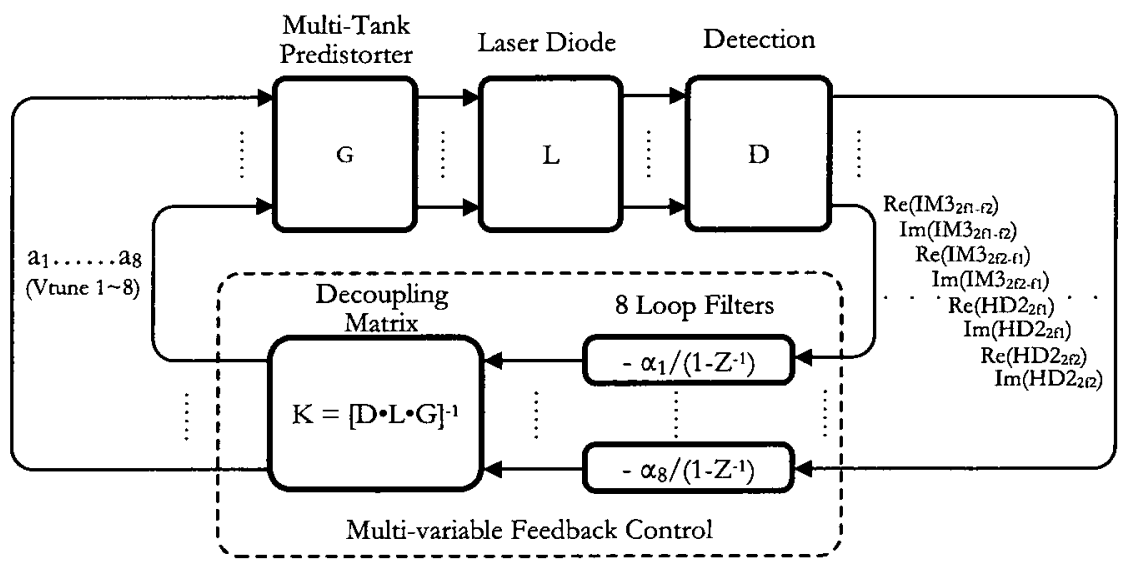

Figure 6.4: Multiple variable feedback. 
Figure 6.3 shows the the block diagram of the predistorter IC and the feedback system. There are eight tank circuits in the predistorter IC. Four tank circuits are used to approximate the frequency profile for the IM3 path and another 4 tank circuits for the HD2 path. The linear path is a delay line used to match the group delay in the predistortion paths. Two RF tones at frequency $f_{1}$ and $f_{2}$ will be fed to the input as the pilot tones for the calibration process. IM3 and HD2 components of the photo-detection output will be detected as vectors, then fed to the feedback control block for adjusting the tuning voltages, therefore minimizing the IM3 and HD2 distortion. This calibration setup is a multiple-input-multiple-output (MIMO) control system. The simplified block diagram of this control system is shown in Figure 6.4. In this block diagram, the predistorter is 
represented by an $8 \times 8$ matrix $-\mathrm{G}$,
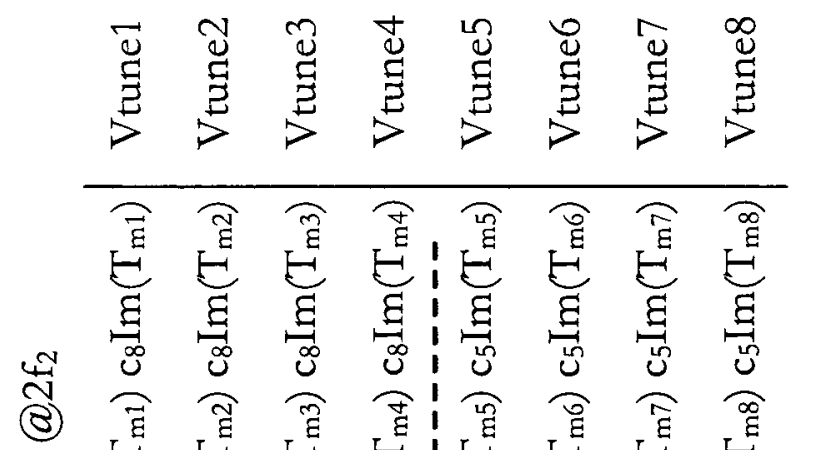

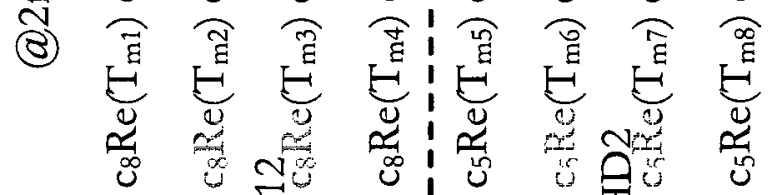

峲

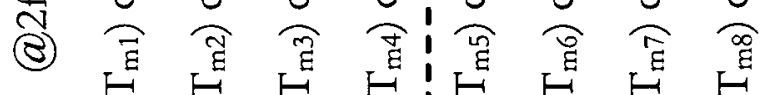

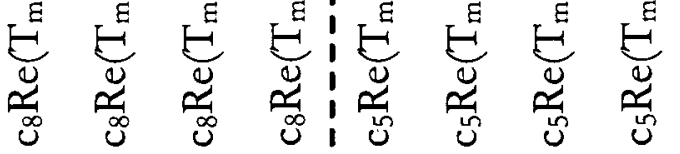

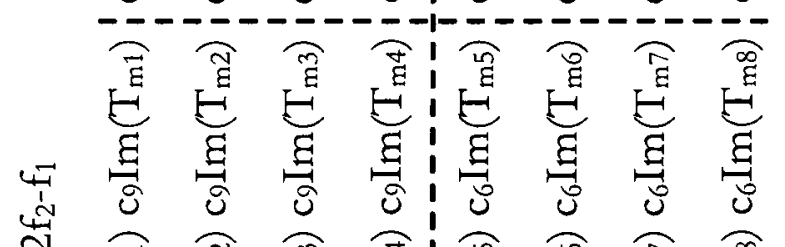

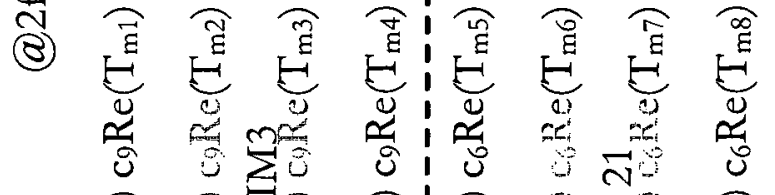

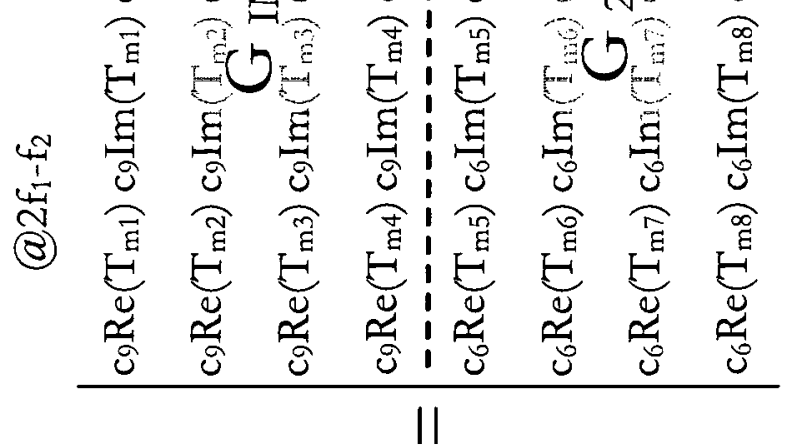

0 
Elements in $G$ are the slopes of the gain versus tuning voltage curve, where $T_{m 1}, T_{m 2}, .$. , $T_{m 8}$ are the responses of the tank circuits at HD2 and IM3 frequencies at the laser driver's output (detailed expression in (4.2.10)). $c_{5}$ and $c_{6}$ are the square and cubic coefficients of the square generator D2. $c_{8}$ and $c_{9}$ are the square and cubic coefficients of the cubic generator D3. The sub-matrices $G_{12}$ and $G_{21}$ of $G$ represent the coupling between the IM3 and the HD2 predistortion path. The matrix $D$ is a diagonal square matrix, used to include the attenuation and phase shift introduced by the detection circuits. The laser diode is represented by block $L$, which describes the nonlinear relationship between inputand output- IM3\&HD2 tones. Considering up to third order distortion, the output of the predistorter contains 12 tones at $f_{2}-f_{1}, 2 f_{1}-f_{2}, f_{1}, f_{2}, 2 f_{2}-f_{1}, 2 f_{1}, f_{1}+$ $f_{2}, 2 f_{2}, 3 f_{1}, 2 f_{1}+f_{2}, 2 f_{2}+f_{1}$ and $3 f_{2}$. At the operating point (given the amplitudes and 
phases of the predistorter output tones), block $L$ can be linearized to an $8 \times 8$ matrix,
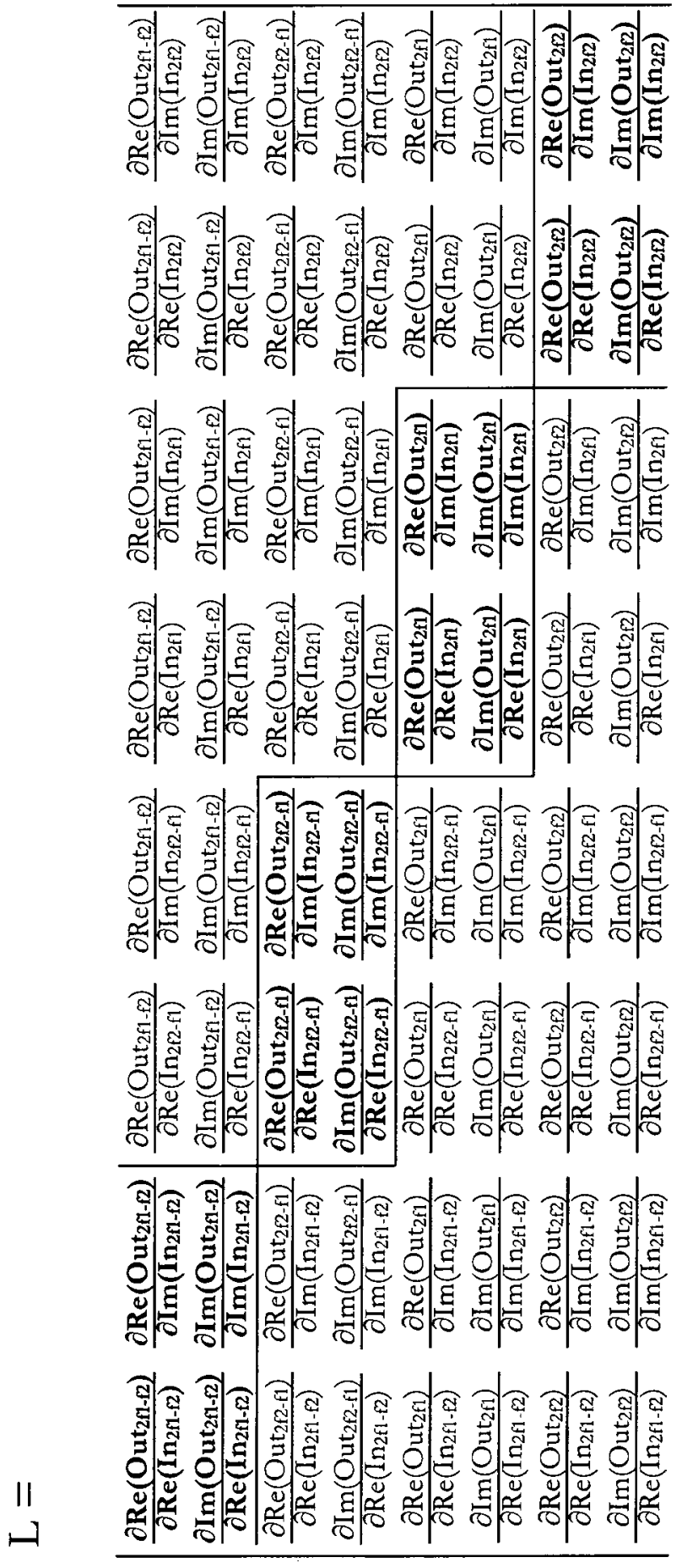
where the $2 \times 2$ sub-matrices along the diagonal line are mostly due to the linear term of the laser input-output function and other sub-matrices reflect the frequency conversion due to the laser nonlinearity. As the nonlinear term of the laser transfer function is much smaller while compared to the linear term, the linear transmission has the dominant control over the output IM3 and HD2 tones. Therefore, the output IM3 and HD2 tones follow the input IM3 and HD2 tones monotonically, which is important for the convergence of the iteration process. Neglecting the frequency conversion terms, the matrix $L$ can be simplified to

$$
\begin{aligned}
& \mathrm{L}=
\end{aligned}
$$

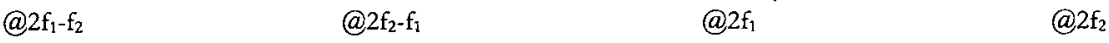

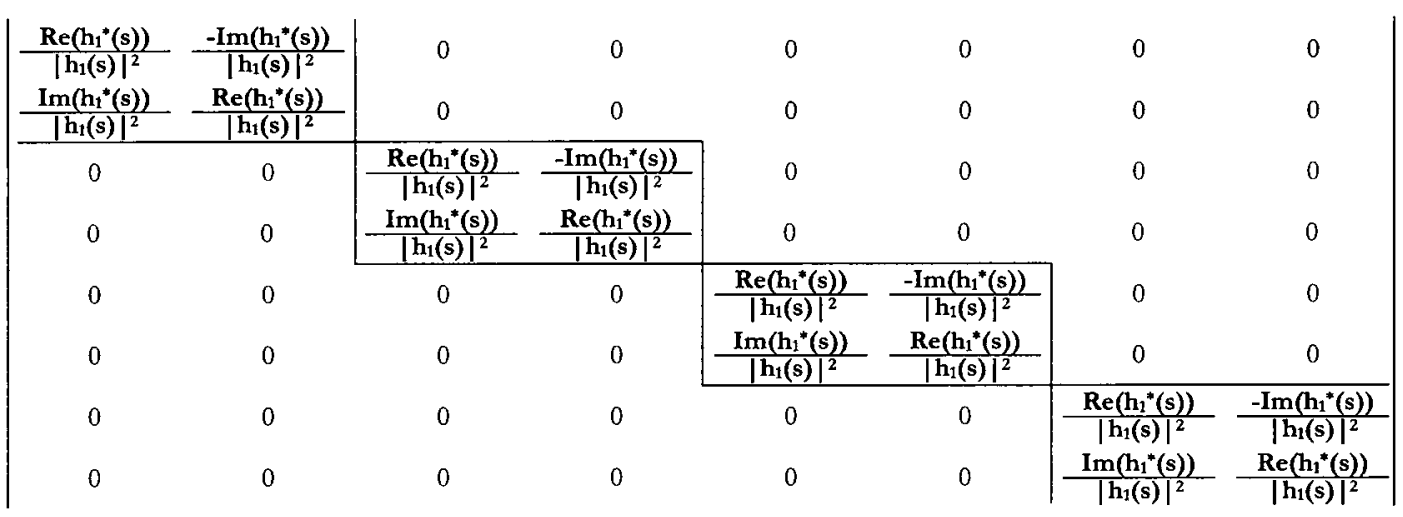

where $h_{1}(s)$ is the linear path transfer function of the laser inverse model (see equation (2.4.5) and (2.5.4)). Decoupling matrix $\mathrm{K}$ is designed as

$$
K=[D L G]^{-1}=\left|\begin{array}{cc}
K_{I M 3} & K_{12} \\
K_{21} & K_{H D 2}
\end{array}\right|
$$

to decouple the interactions among the multiple control variables. The overall system under control becomes

$$
D L G=I
$$

where $I$ is unit diagonal matrix. Therefore diagonal control [77] can be applied, which simplifies the loop filters into eight separate branches and design methods for the singleinput-single-output (SISO) system can be used. In this thesis, simple accumulators are used as the loop filters, which corresponds to the fixed-point iteration illustrated in Figure 
6.2. As the overall system under control is represented as a diagonal unit matrix, the slope of distortion versus input control curve is 1. Relaxation factors $\alpha_{i}$ are then applied as the fixed slope used in the iteration process for adjusting the overshoot of the control signal and assisting the convergence. The sub-matrices $K_{12}$ and $K_{21}$ of $K$ can be reset to zeros to achieve decentralized feedback control [77], therefore simplifying circuit implementation.

\subsection{IC Layout and Micrograph}

The silicon area used for the chip is $1.2 \mathrm{~mm} \times 1.2 \mathrm{~mm}$. While this seemed enough at first, it turns out to be a challenging task to fit the circuit within that space. This is mostly due to the number of pads and dc-decoupling capacitors required in the circuit.

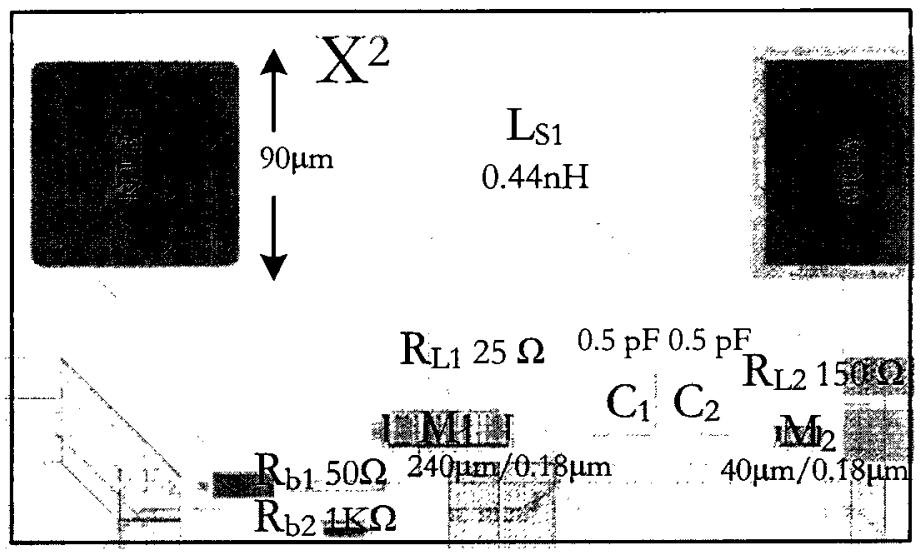

Figure 6.5: Layout of the $X^{2}$ component.

Figure 6.5 shows the layout of the $X^{2}$ component. The pad sizes are $90 \mu m \times 90 \mu m$. Due to the limited chip area, one pad was removed for placing the peaking inductor. The $\mathrm{RF}$ input pad directly connects to the gate of transistor $M_{1}$. The dc-blocking capacitor for the RF input is placed off-chip on the test board. $R b_{1}$ is the bias resistor for the RF input, which is $50 \Omega$ and also provides the input matching. $R b_{2}$ is the bias resistor for transistor $M_{2}$. To stabilize the bias voltage at the gate and the output voltage at the drain of $M_{2}$, dc-decoupling capacitors $C_{1}$ and $C_{2}$ were placed close to transistor $M_{2}$. Figure 6.6 shows the layout of the LC tank circuit, the tunable Gilbert cell and the laser driver. MIM 


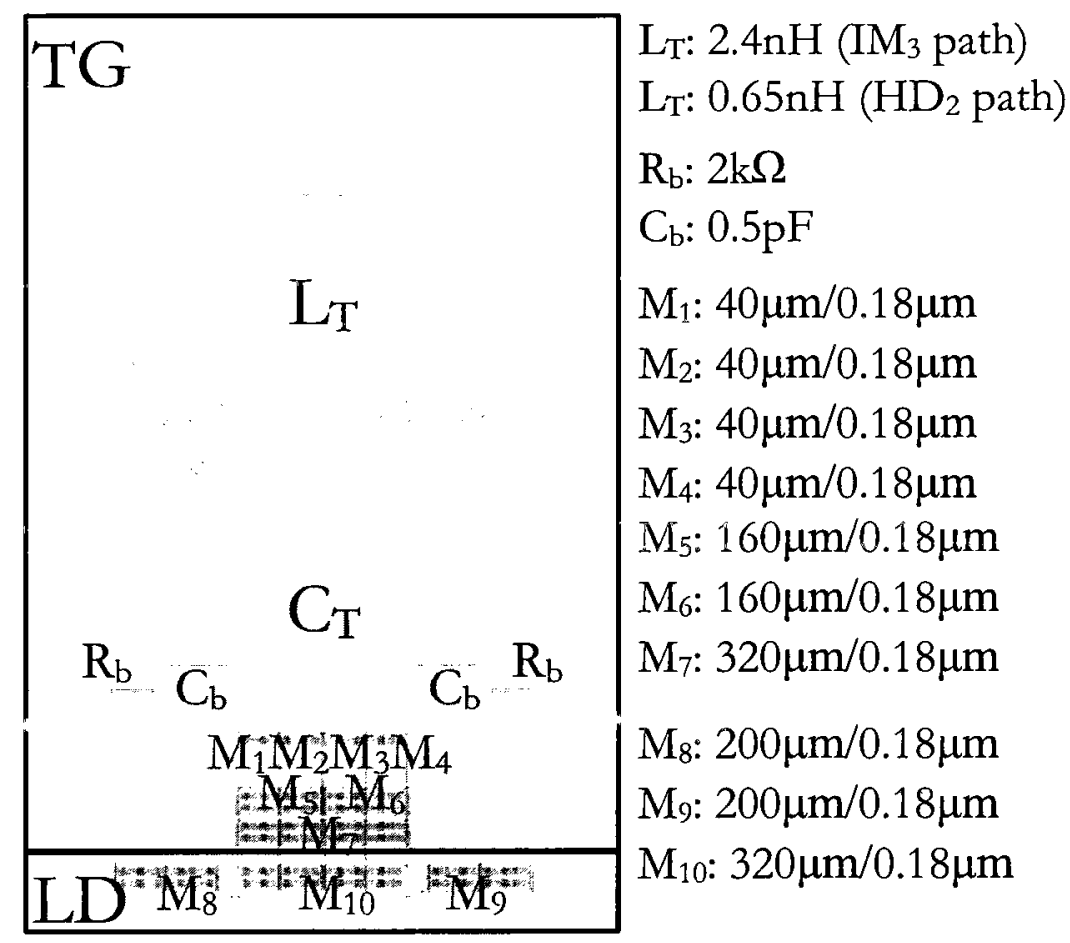

Figure 6.6: Layout of the tunable gain tank circuit and the laser driver.

capacitors $\left(C_{T}\right)$ were used in the $\mathrm{LC}$ tank because of the linearity requirement. Centertapped spiral inductors $\left(L_{T}\right)$ were used to maximize the symmetry and utilize the virtual ground at the center tap. The tunable gain tank circuit (TG) connects to the laser driver through coupling capacitors $\left(C_{b}\right)$. The gate bias voltages of the differential pair in the laser driver are provided through $R_{b}$. This gives the flexibility of adjusting the laser driver input bias independently. For tuning voltages and bias voltages provided off-chip, N-diodes were used for ESD protection and dc-decoupling. Differential signal lines were laid out in parallel to reduce common-mode interference. Figure 6.7 shows the wire connection of the laser driver outputs. The metal widths of the lines were properly designed according to the layout design rules for accommodating the large running current. The complete chip layout is depicted in Figure 6.8 and the micrograph of the fabricated chip is shown in Figure 6.9.

In this layout, there are a few potential problems. For example, dc-coupling capacitance 


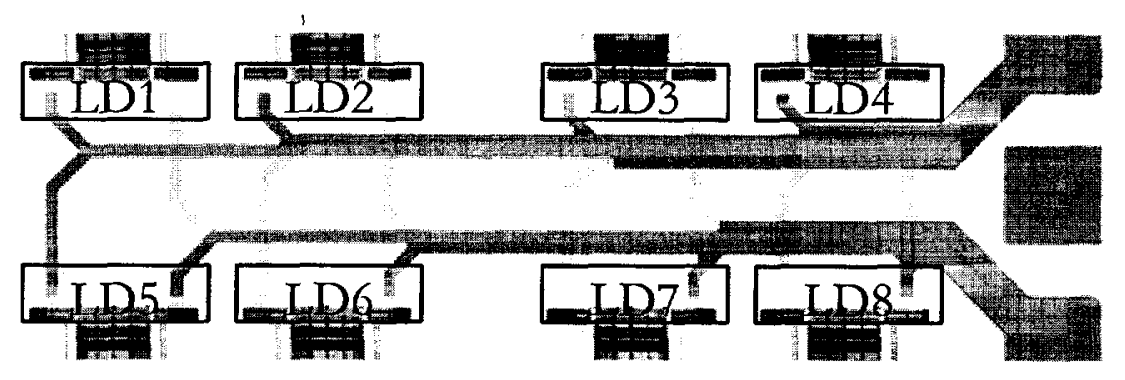

Figure 6.7: Layout of current summing circuit.

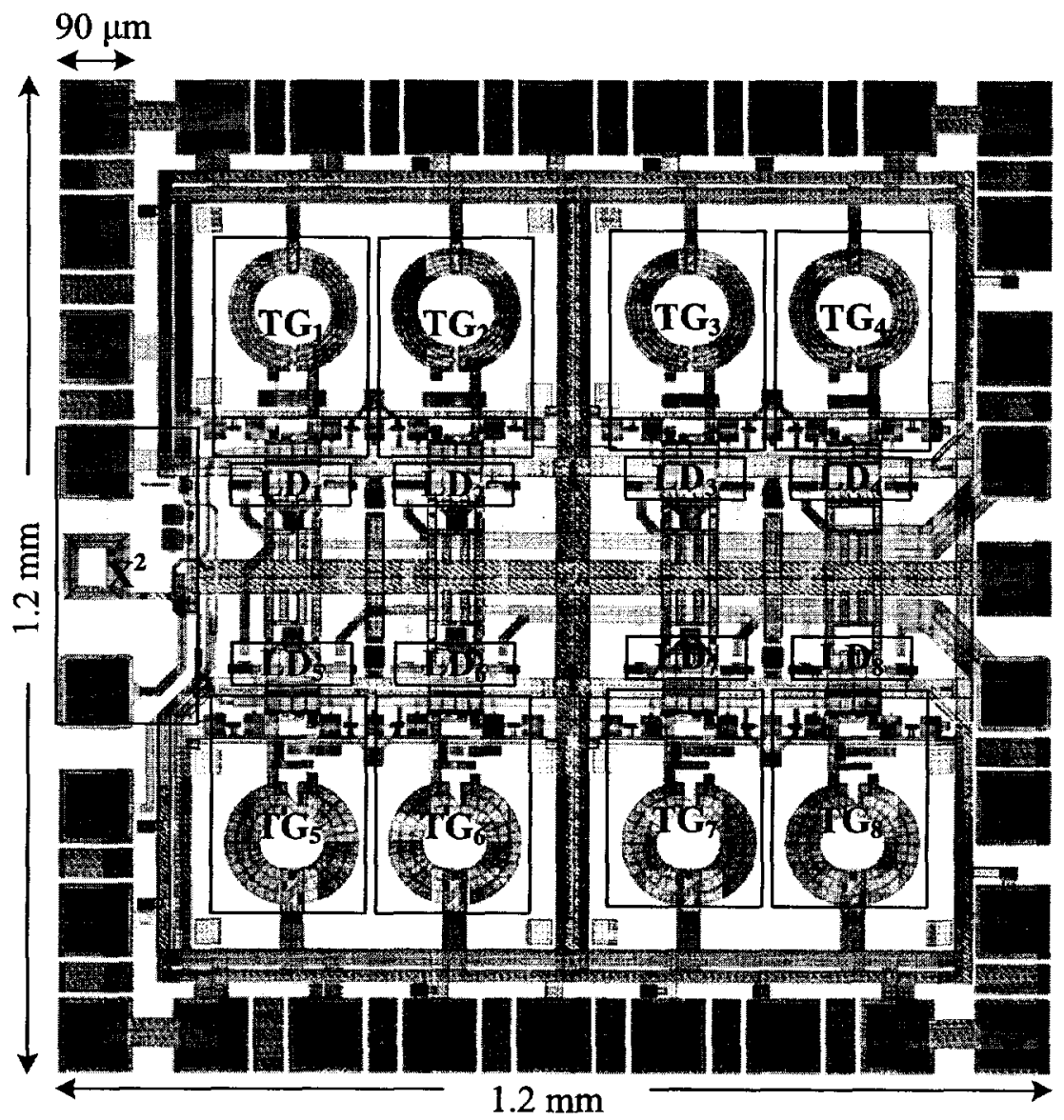

Figure 6.8: Complete layout of the predistorter IC. 


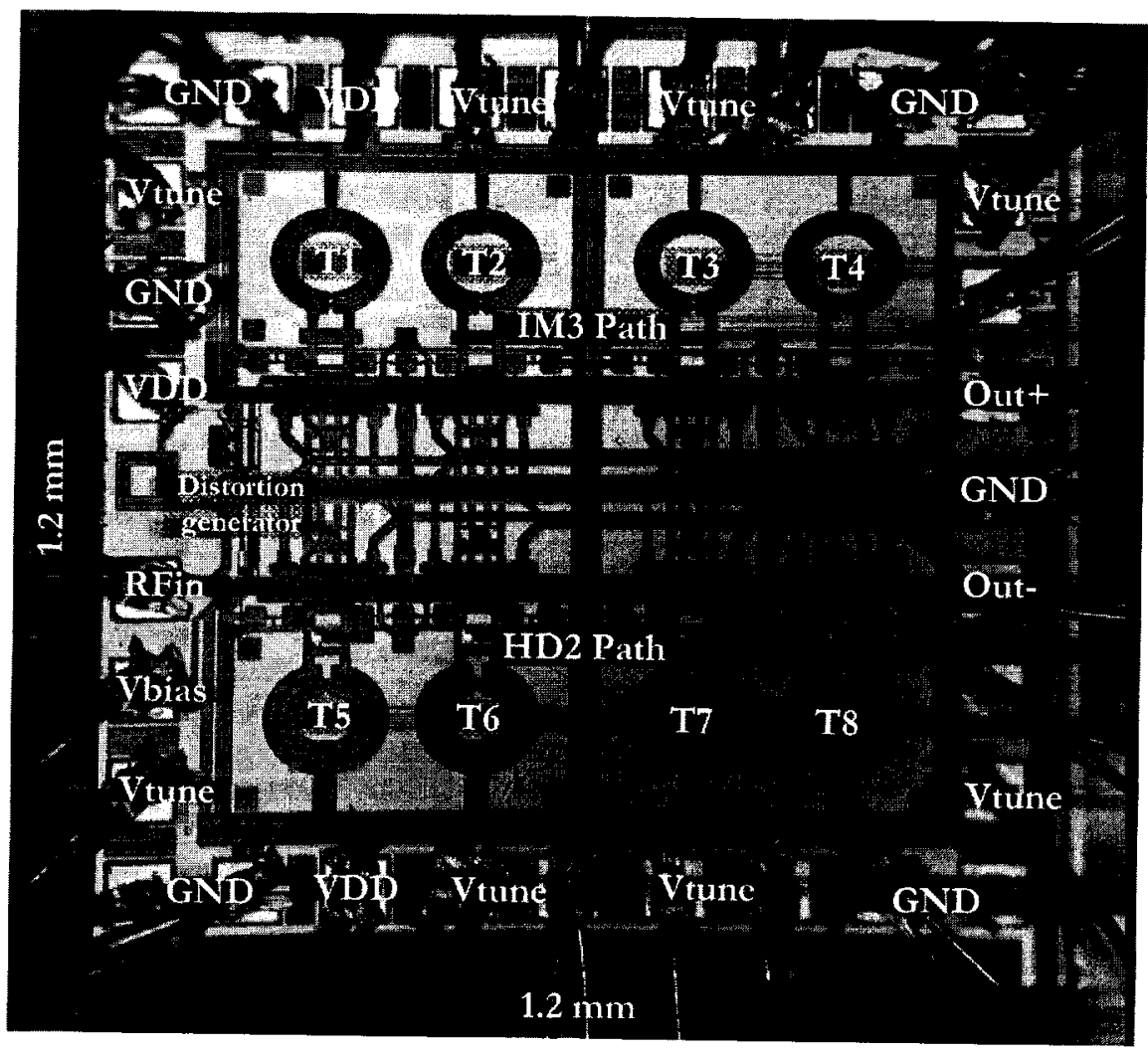

Figure 6.9: Micrograph of the fabricated predistorter IC. 
was not sufficiently provided due to the limited chip area. Also, the layout is crowded, the mutual coupling between the inductors was not considered in the design stage. Identical finger geometry was used to lay out all the transistors for a better transistor size matching. However, other matching techniques, such as common-centroid and interdigitated layout, were not used. This layout potentially suffers from gradient-induced mismatches due to diffusion and etch variation and thermal and stress effects.

\subsection{Chip Bonding and Test Board Design}

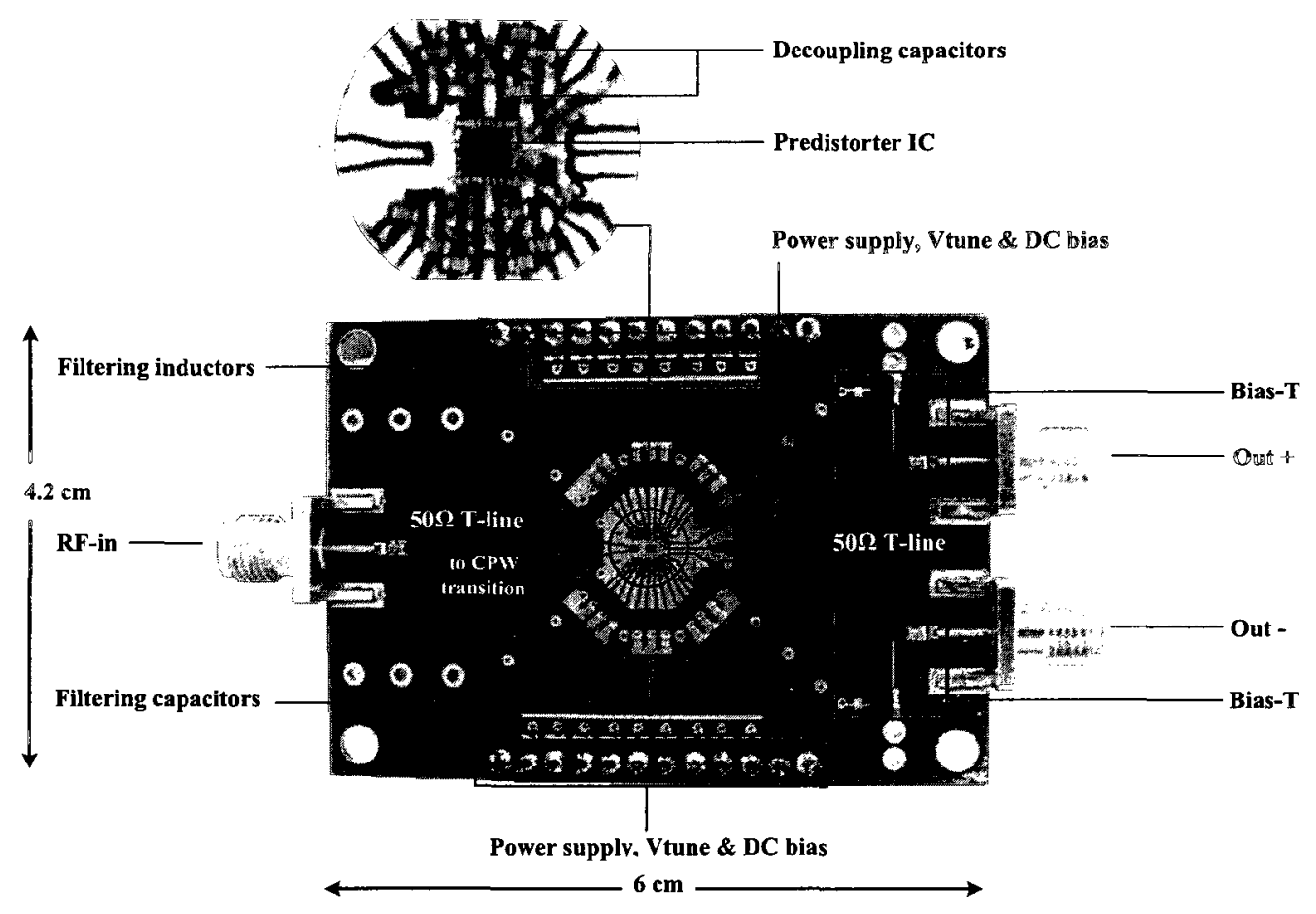

Figure 6.10: Photograph of the test board with a zoomed-in view of the chip bonding area.

In order to test the prototype predistorter circuit, a custom PCB was designed and is shown in Figure 6.10. The bonding leads on the PCB are 7 mil wide with 7 mil clearance. Gold wires were tightly bonded from the the chip to the PCB to reduce the bond-wire inductance. Decoupling capacitors were used for stabilizing the tuning voltages and the bias voltages. Surface mount capacitors with the smallest package (0201) were placed as close to the 
chip as possible to enhance the decoupling. Filtering inductors and capacitors were used to remove noise or any interference from the voltage sources. Both input and output RF signal lines were carefully designed for a wideband matching to the $50 \Omega$ measurement system. Bias-T circuits were implemented on the PCB to provide power supply for the laser driver stage on the chip. Both input and output RF ports use edge-launched SMA connectors for their wideband performance.

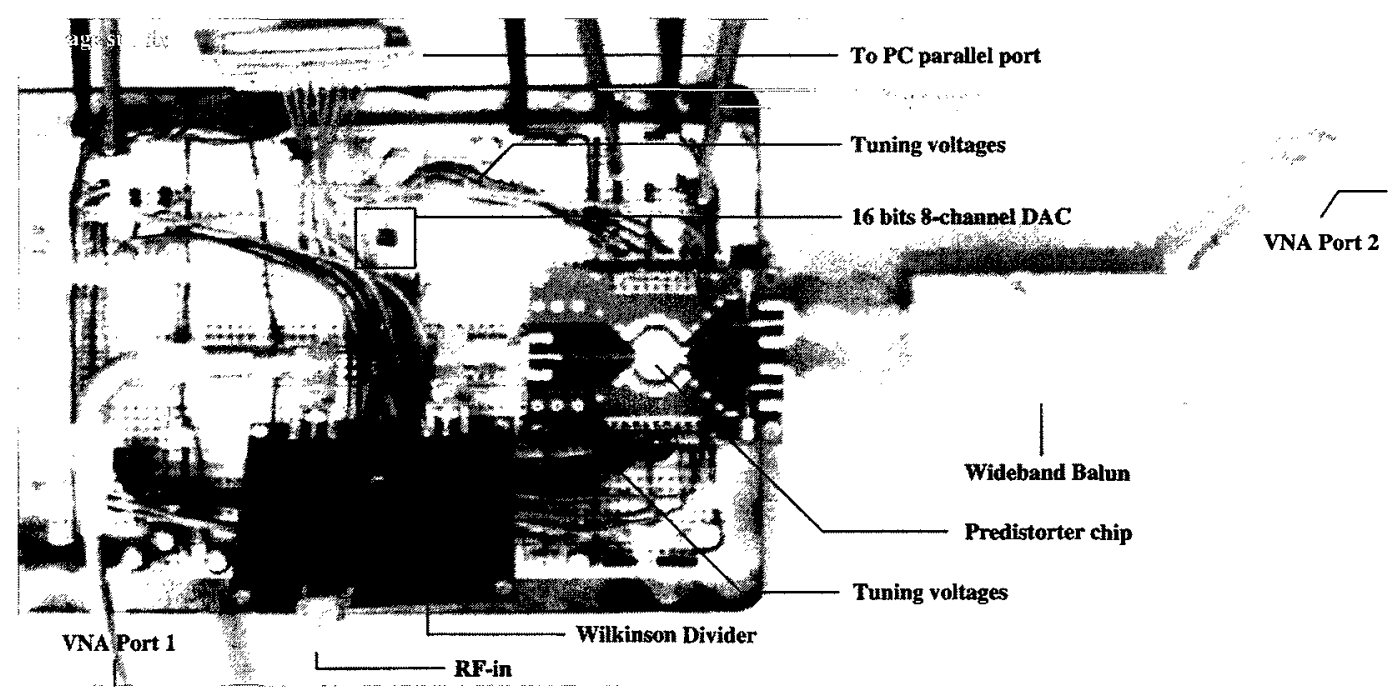

Figure 6.11: Picture of the test board mounted on a bread-board.

The differential outputs from the test board connect to a wideband balun (described in Chapter 5), which combines the outputs to a single-ended output. The test board was mounted on a bread-board with a 16-bits 8-channel DAC (Analog Device AD5668), which provides the eight tuning voltages of the predistorter. The DAC's clock and data input pins were connected to a PC's parallel port, which was programmed to mimic the control sequence of configuring the DAC. Trimmer potentiometers were used on the bread-board to provide a few bias voltages. 


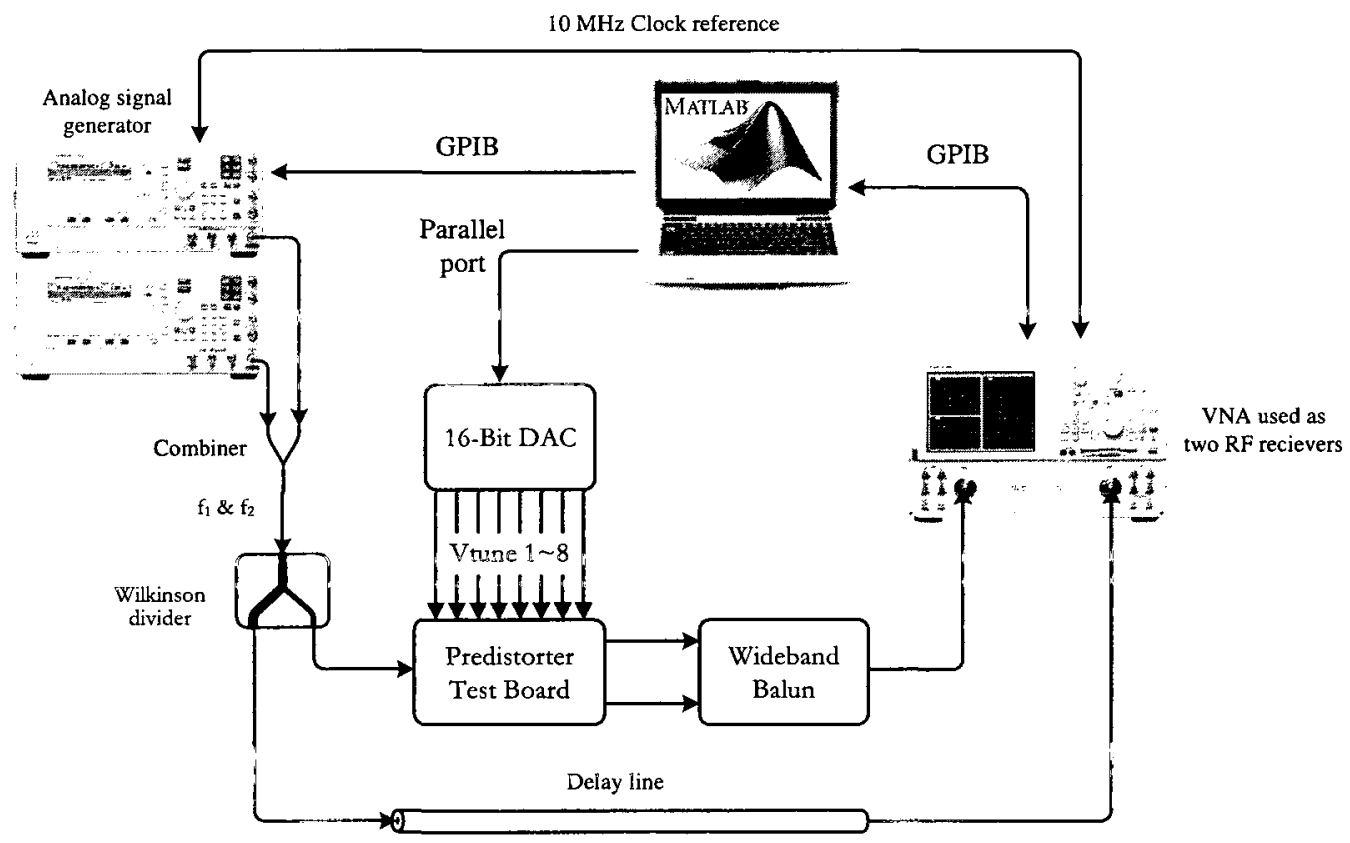

Figure 6.12: Test setup.

\subsection{Test Setup and Equipments}

The block diagram shown in Figure 6.12 provides an overview of the test setup. Two analog signal generators (Agilent PSG E8257D) were used to generate the two input RF tones. A Wilkinson divider was used to deliver the input signal to the linear branch and the predistortion branch. A Vector Network Analyzer (VNA) capable of harmonic and intermodulation measurements (Agilent PNA N5230A $[78,79]$ ) was used. Each of the VNA's two ports has a RF receiver, which was used to detect the outputs from the predistorter. In the linear branch, a coaxial cable was used as the delay line and connected to one port of the VNA. The predistorter IC's differential output was combined by a wideband balun and connected to the other port of the VNA. The signal generators are synchronized to the VNA through the $10 \mathrm{MHz}$ clock reference. Eleven output tones of the predistortion circuit at $2 f_{1}-f_{2}, f_{1}, f_{2}, 2 f_{2}-f_{1}, 2 f_{1}, f_{1}+f_{2}, 2 f_{2}, 3 f_{1}, 2 f_{1}+f_{2}, 2 f_{2}+$ $f_{1}$ and $3 f_{2}$ were measured by the VNA and acquired by a computer running MatLab through the GPIB port of the equipment. The laser model and the adaptive calibration 
algorithm were implemented in the MatLab environment. The tuning voltages of the predistorter circuit were provided by a 16-bit 8-channel DAC (Analog Device AD5668), which is controlled through the computer's parallel port and programmed in the MatLab environment. The computer also connects to the signal generators through the GPIB port for configuring the signal generators in the MatLab environment. Spectrum analyzer (Agilent PSA E4440A) was also used to observe the input and output signal spectrum.

\subsection{Measurement}

\subsubsection{Input Signal}

Figure 6.13 shows the spectrum of the input signal at the input of the predistorter IC. One tone is at $1.98 \mathrm{GHz}$ and the other tone is at $2.02 \mathrm{GHz}$. These two tones are chosen at the center of the frequency band with $40 \mathrm{MHz}$ separation and can be easily distinguished on the spectrum analyzer. They are used as the pilot tones for calibrating the predistorter. The calibration process will optimize the frequency profile around the band center. It relies on the interpolation by the tanks to extend the linearized bandwidth. The input signal power is $-16 \mathrm{dBm}$, which corresponds to the amplitude of $50 \mathrm{mV}$ in a $50 \Omega$ measurement system. It is near the region where the peak of the square term of the distortion generator is obtained. The signal to the noise floor is better than $85 \mathrm{~dB}$ and no spurious tones at $2 f_{1}-f_{2}$ and $2 f_{2}-f_{1}$ are observed. The two input tones were generated by two analog signal generators. One demonstrates very low harmonic distortion, the other one generates a second order harmonic tone which is $46 \mathrm{~dB}$ below the input signal. In theory, the predistorter will blindly try to remove the distortion even from the input. However, because the predistorter designed relies on the interpolation by the tank circuits, it is not possible to remove the one harmonic tone in the input, as this requires an abrupt change of the frequency profile between $2 f_{1}$ and $2 f_{2}$. This harmonic tone will have an effect on the convergence of the feedback control, as will be observed in the calibration shown in the following sections. 


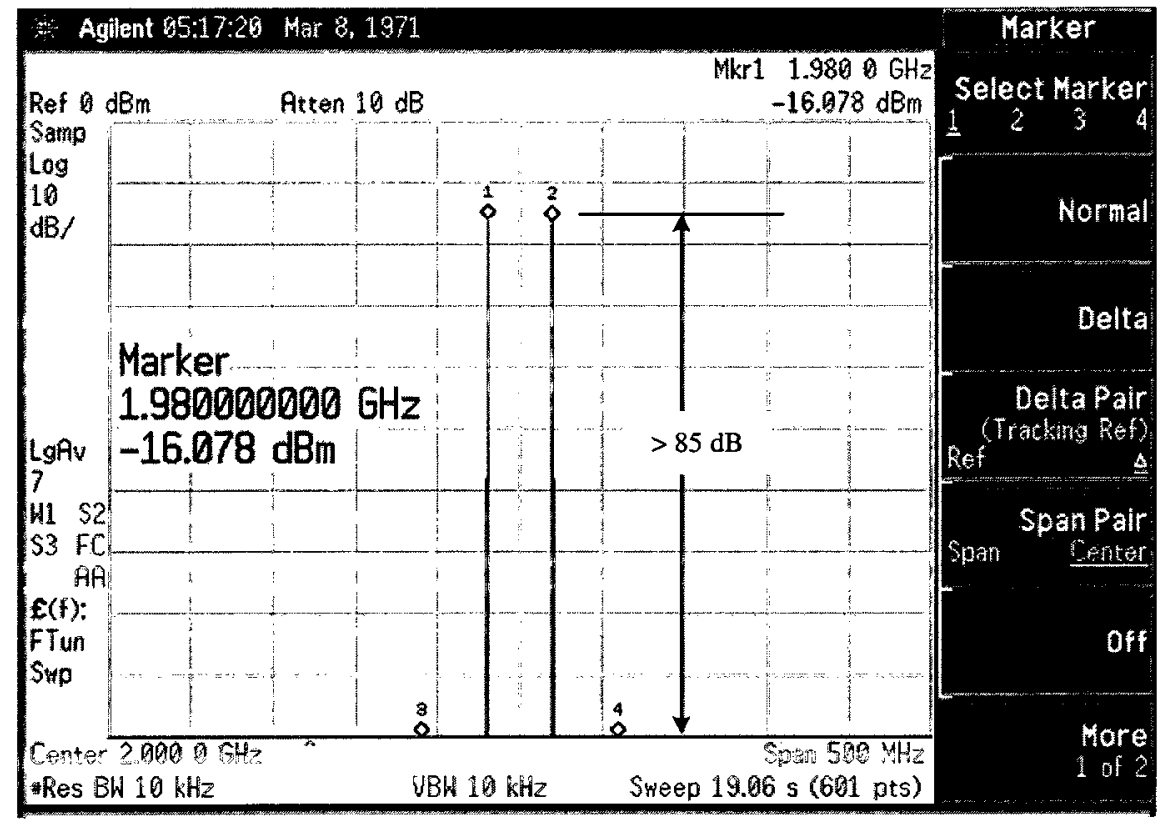

(a) $1.75-2.25 \mathrm{GHz}$

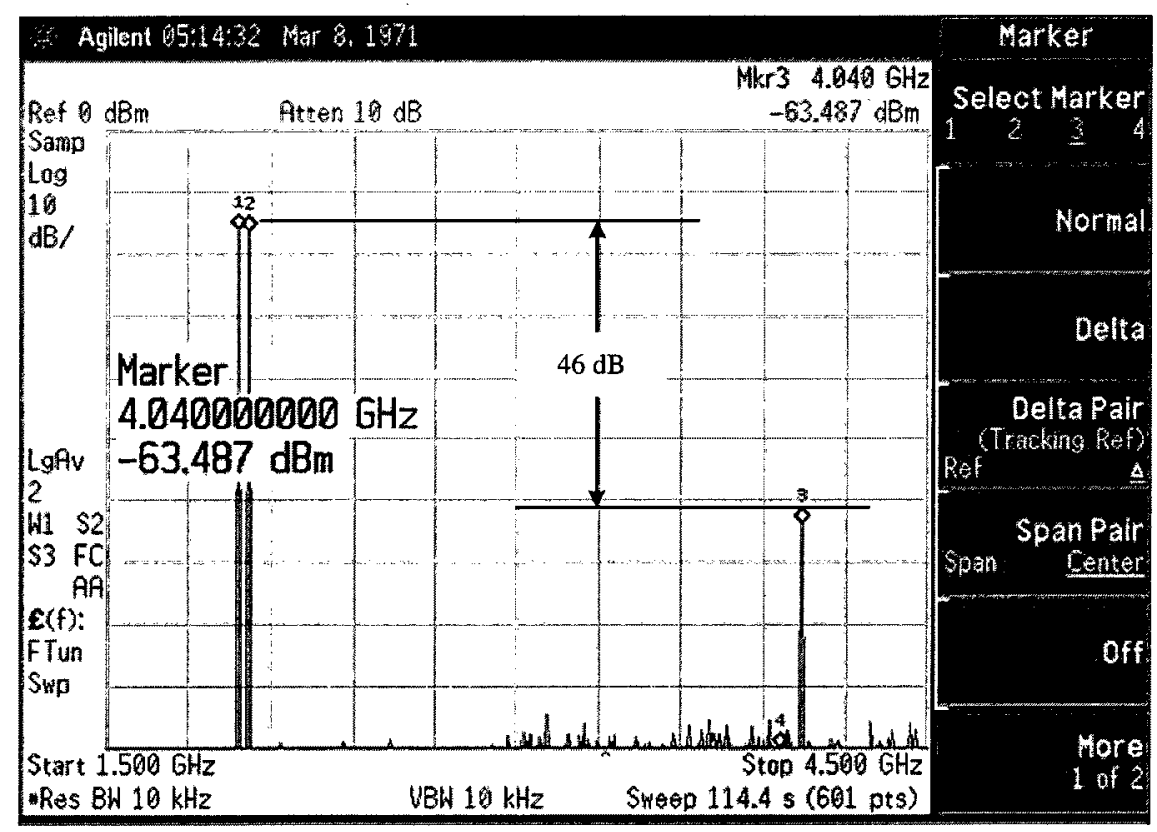

(b) $1.5-4.5 \mathrm{GHz}$

Figure 6.13: Spectrum analyzer screenshot showing the two-tone input signal. 


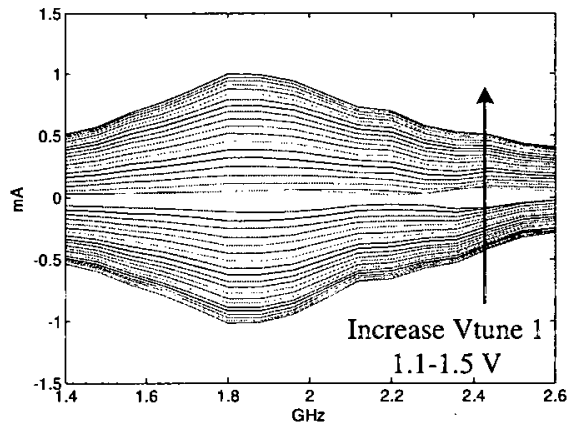

(a)

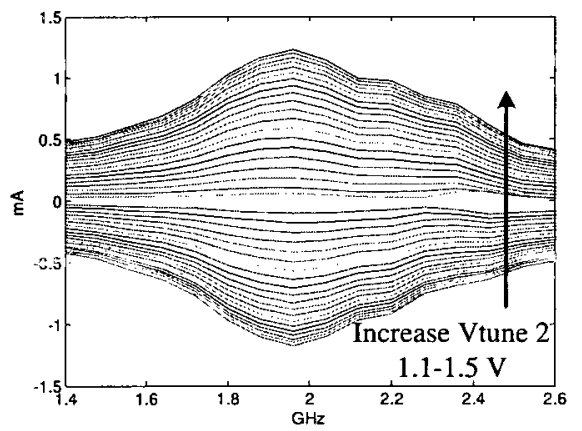

(b)

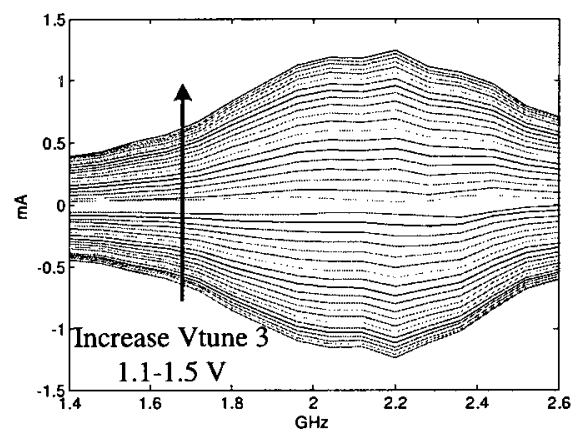

(c)

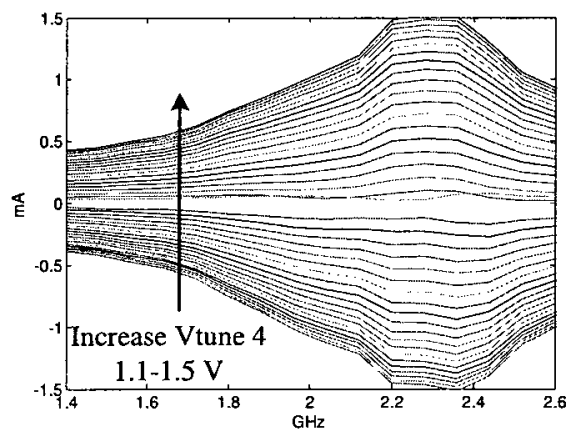

(d)

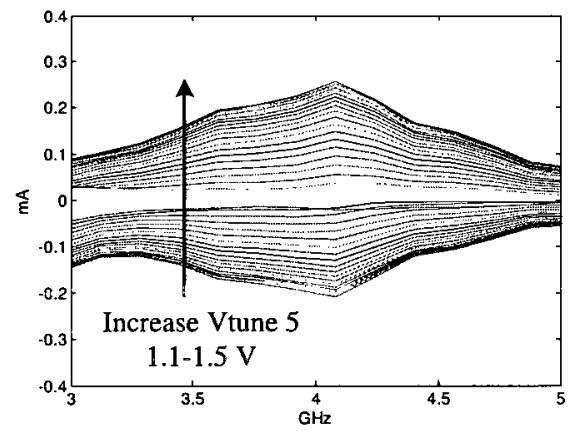

(e)

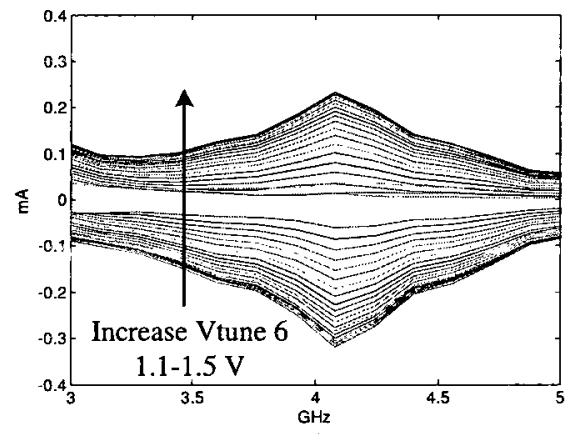

(f)

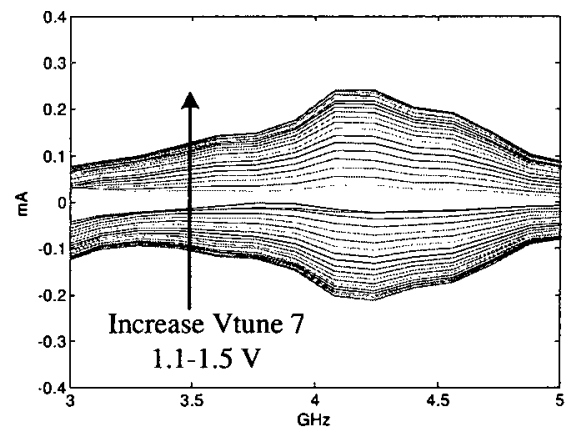

(g)

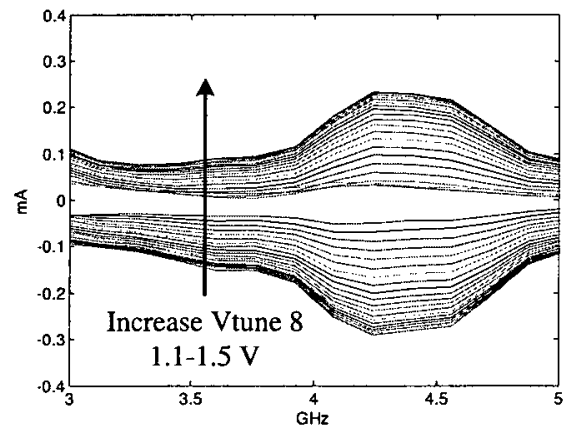

(h)

Figure 6.14: Measured results showing the tank circuit alignment and tuning. The tuning voltages were increased from 1.1 to $1.5 \mathrm{~V}$ in $0.01 \mathrm{~V}$ step to demonstrate the fine tuning capability. 


\subsubsection{Responses of the Tank Circuits}

To observe the responses of the tank circuits, a single-tone input was swept from 1.4-2.6 GHz and 3-5 GHz for IM3-band and HD2-band tank circuits respectively. The measured predistorter IC output magnitudes (in $\mathrm{mA}$ ) are shown in Figure 6.14. The tuning voltages were adjusted from 1.1 to $1.5 \mathrm{~V}$ in $0.01 \mathrm{~V}$ step to demonstrate the fine tuning capability. The results are plotted on the negative side of the graph for the tuning voltages from 0.9-1.3 $\mathrm{V}$ and on the positive side for the tuning voltages from 1.3-1.5 V. The tank circuits are functioning, but the shapes of the responses are not exactly what an ideal tank circuit should have. The bandwidth also varies for each tank circuit. The results seem quite far away from expectations. However, the results exhibit certain features, which leads to a good interpolation of the desired frequency profile for the predistortion purpose. The center frequencies of the tank circuits are spaced apart. Therefore, the tuning voltages are linearindependent to each other for frequency profile approximation by vector combination. The tuning voltages control the gains in a monotonic way, which is necessary in feedback control. The tank circuit center frequencies for the IM3-band are around $2 \mathrm{GHz}$ as expected. The tank circuits frequencies for the HD2-band should be around $4 \mathrm{GHz}$, however they are shifted towards higher frequencies. This is partially due to the mutual coupling between the inductors. In the layout, inductors are placed closely to each other. As the current rotations of the two neighbor inductors are in the same direction, the mutual inductance is negative, which reduces the inductance in effect. Therefore, the tank circuit center frequencies are shifted to higher frequencies.

\subsubsection{Adaptive Calibration}

\section{K Matrix and Gain Saturation}

In the design stage, the derivation of the decoupling matrix $\mathrm{K}$ can be obtained using the definition of 6.2.5. However, because the tank circuits' responses of the prototype IC are quite away from the expected ideal response, the $\mathrm{K}$ matrix to be used in the calibration process is estimated by measurements. First, the matrices multiplication $D L G$ is assessed 
at the starting point of the tuning voltages. The elements in matrix $D L G$ is the derivatives of the output HD2 and IM3 distortion with respect to the each tuning voltage. They are estimated by measuring the distortion changes with a small increment of each tuning voltage. The measured $D L G$ matrix is then inverted and decentralized to get the $\mathrm{K}$ matrix. The feedback algorithm is then applied using this $\mathrm{K}$ matrix.

Figure 6.15 shows the settling process of the adaptive calibration. The RF tones of $5 \mathrm{~mA}$ amplitude are used as the input of the laser model. It can be observed that the calibration process reduces the IM3 distortion successfully. However, the improvement on HD2 distortion is not great. This is because the HD2 distortion level is high and the predistorter's square path can not provide enough distortion cancelation. By observing the tuning voltages, it can be seen that the tuning voltages are more than 2 Volt, which is out of the linear region the gain cells (see the voltage range in Figure 4.9). To reduce the HD2 distortion level, the $\mathrm{RF}$ pilot tones was reduced to $3 \mathrm{~mA}$ amplitude and the settling process is shown in Figure 6.16. More than $10 \mathrm{~dB}$ improvement on HD2 distortion reduction was observed.

The purpose of the K Matrix is to decouple the interactions among each control loop, so that the feedback control works independently in each loop. However, the $\mathrm{K}$ matrix is estimated at the starting point, it will be mismatched to the predistortion system when the tuning voltages are away from the starting point. This is due to the nonlinearity of the tunable gain and the nonlinearity of the laser, especially when the tuning voltages are into the gain saturation region. Because of this mismatch, the control signal from one loop leaks to other loops and affects the convergence of their settling process. 


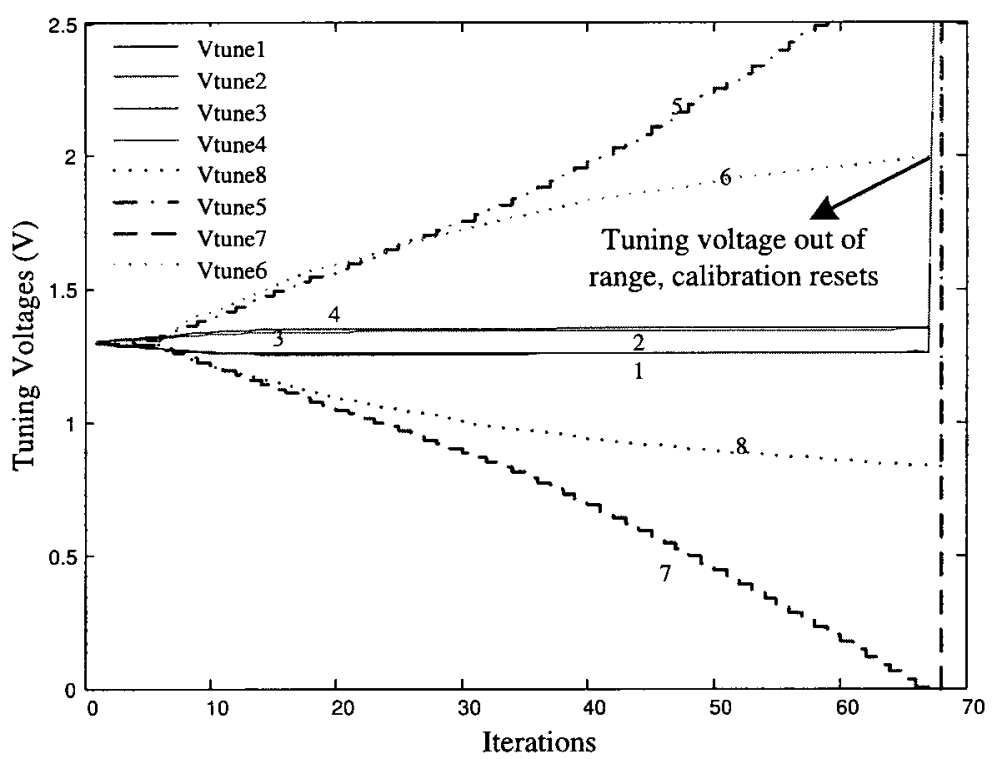

(a)

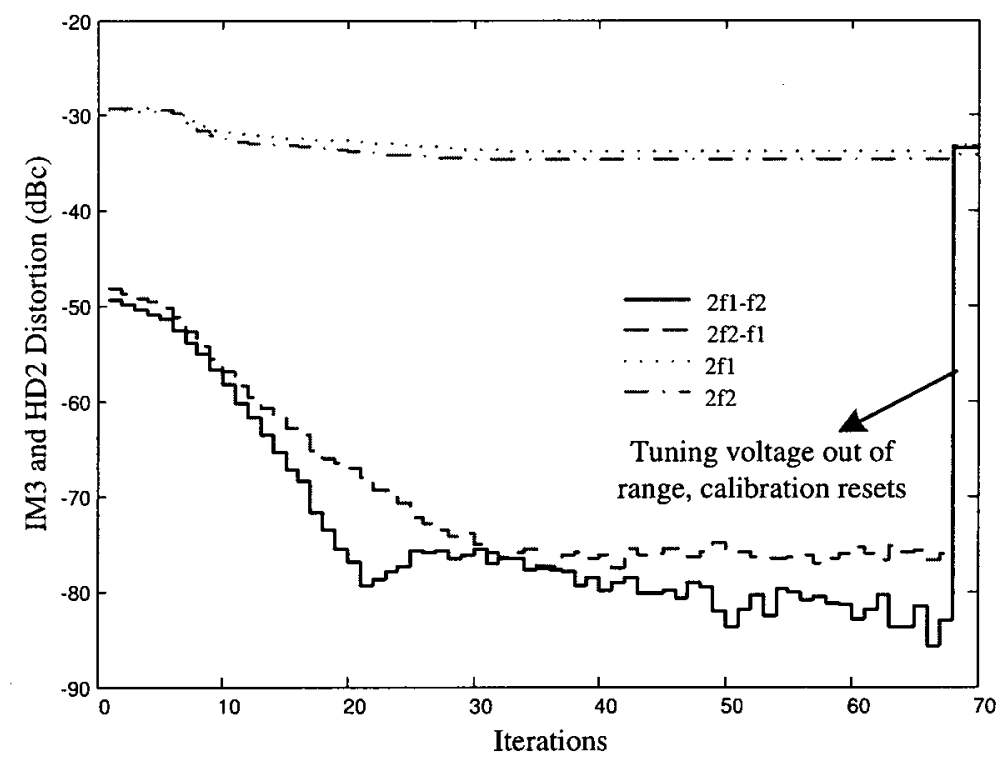

(b)

Figure 6.15: Measured results showing the calibration process with $5 \mathrm{~mA}$ RF input to the laser. 


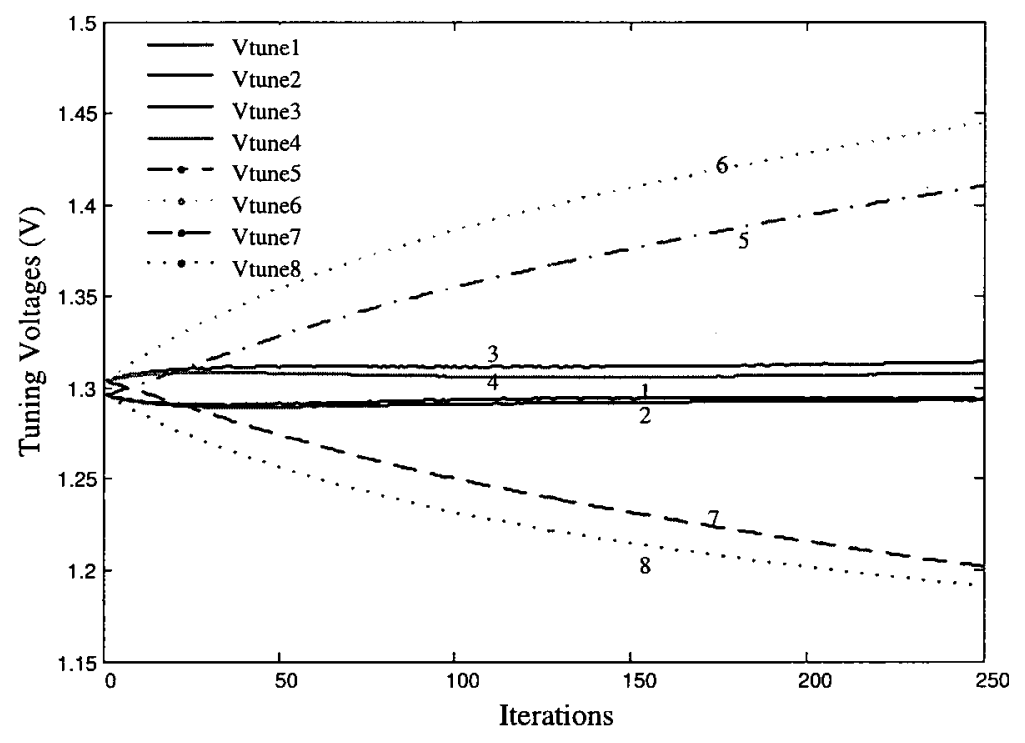

(a)

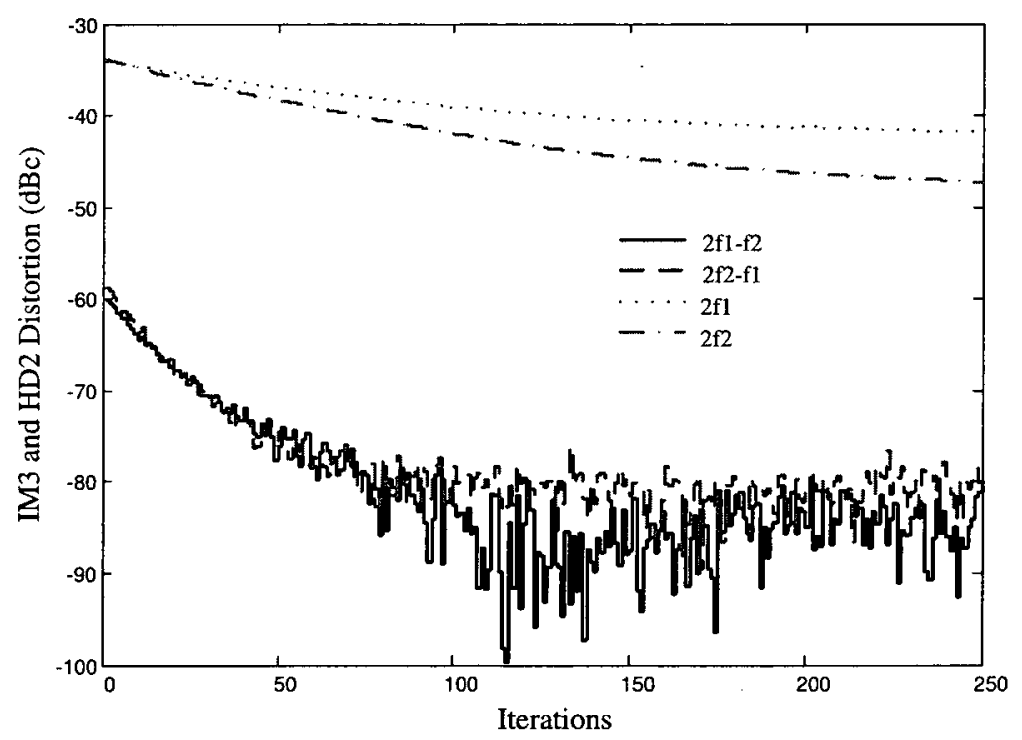

(b)

Figure 6.16: Measured results showing the calibration process with $3 \mathrm{~mA}$ RF input to the laser. 


\section{Relaxation Factor}

In the feedback control loops, simple accumulators are used as the loop filters. The relaxation factors $\alpha_{i}$ are used to adjust the convergence of the calibration process. Apparently, a large $\alpha$ speeds up the convergence. However, it brings a potential problem of overshooting the tuning voltages, which might be out of the convergence region and cause instability. These phenomenon is conceptually illustrated in Figure 6.17.

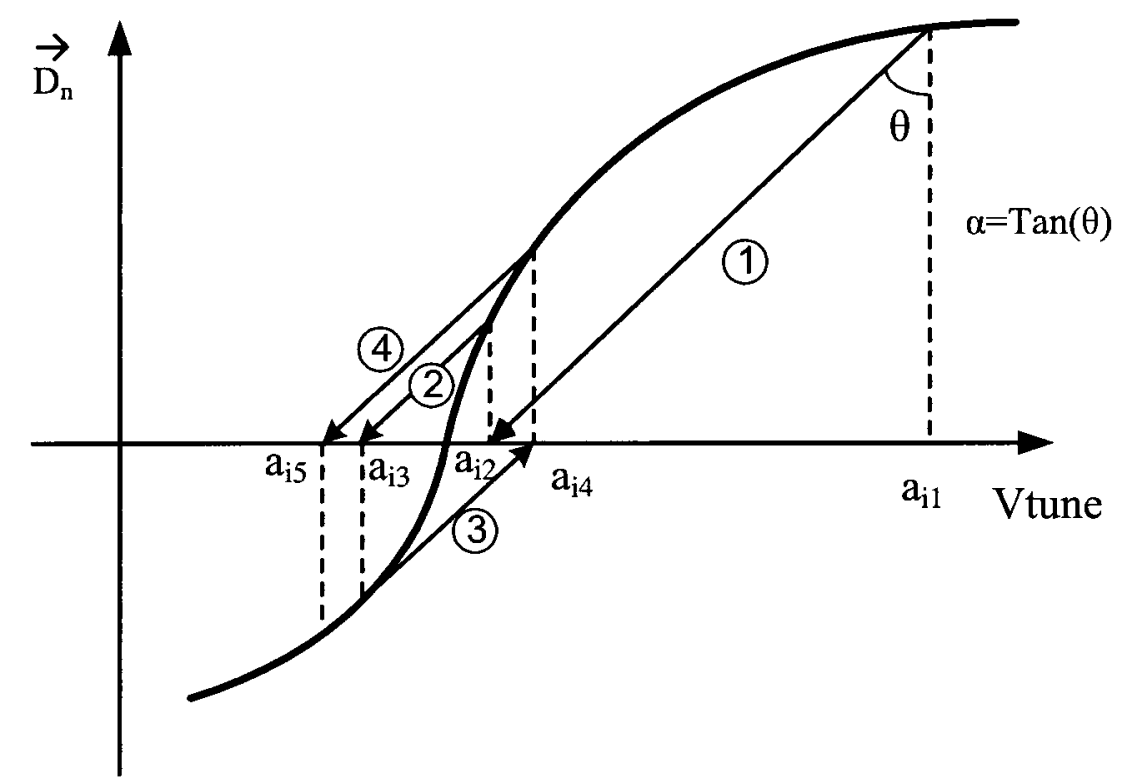

Figure 6.17: Conceptual illustration of the feedback instability due to a large relaxation factor.

When the tuning voltages are away from the reference voltage (defined as $1.3 \mathrm{~V}$ shown in Figure 4.7), the tunable gain cells enter the gain saturation region. This is represented by a compressive function of the distortion response. From Figure 6.17, it can be seen that if the relaxation factor is too large the tuning voltage during the iteration will pass the convergence point and then go back again creating an oscillation behavior. Depending on the shape of the response curve, this oscillation could be ever-lasting or even increasing. 
To avoid this problem, the relaxation factor should be less than the slope of the distortion response curve at the convergent point. By this way, the tuning voltages will not pass but gradually approach the convergent point.

As mentioned in the previous section, the $\mathrm{K}$ matrix is estimated at the starting point, it will not exactly match to the predistortion system when the tuning voltages are away from the starting point. Because of this $\mathrm{K}$ matrix mismatch, the control signal from one loop leaks to other loops and affects the convergence of other loops. The HD2 distortion is much higher than the IM3 distortion, the leakage of the HD2 control signal is much stronger than the leakage of the IM3 control signal. Therefore, it is preferable to reduce the relaxation factor of the HD2 control loop and let the HD2 distortion control settles after the IM3 distortion control.

To observe the effect of the relaxation factor, the relaxation factors $\alpha$ for the HD2 control are increased from 0.01 to 0.02 . The settling process is shown in Figure 6.18. Comparing to the settling process shown in Figure 6.16, the tuning voltages for the HD2 control changes faster and the distortion reduction has improved by about $7 \mathrm{~dB}$. Increasing the relaxation factor of the $\mathrm{HD} 2$ control affects the convergence of the IM3 control because of the signal leakage due to mismatch. Figure 6.19 shows the settling process of increasing the $\mathrm{HD} 2$ loop relaxation factors $\alpha$ to 0.06 . Due to the detection noise, the distortions settle to the noise level and change randomly around the noise level. Also, due to the existence of one second order harmonic tone at the input, the HD2 distortions are reduced to the level of the input HD2 component and fluctuate around this level. Due to this fluctuation, the tuning voltages of the HD2 control loops do not settle to fixed values but vary around. Due to the mismatch leakage, the tuning voltages of the IM3 control loops are also affected.

\section{Tracking}

The behavior of semiconductor laser changes from component to component and fluctuates due to temperature variation and aging effect. The calibration should be able to track the laser's behavior variation and provide distortion correction for the lasers over time and temperature fluctuation. 


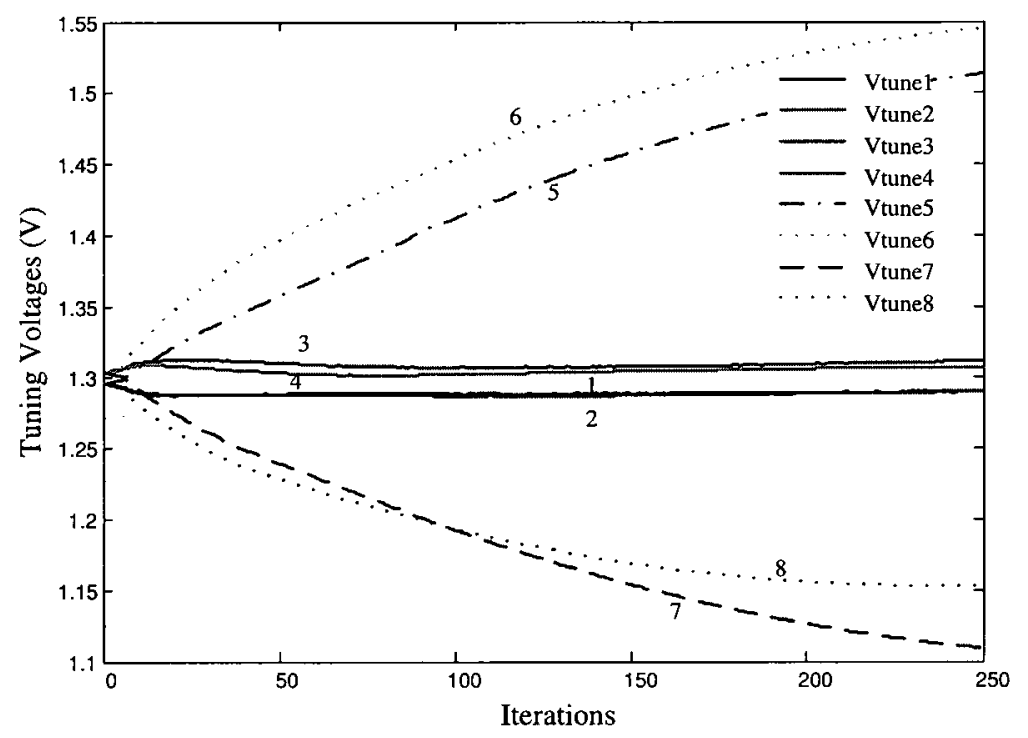

(a)

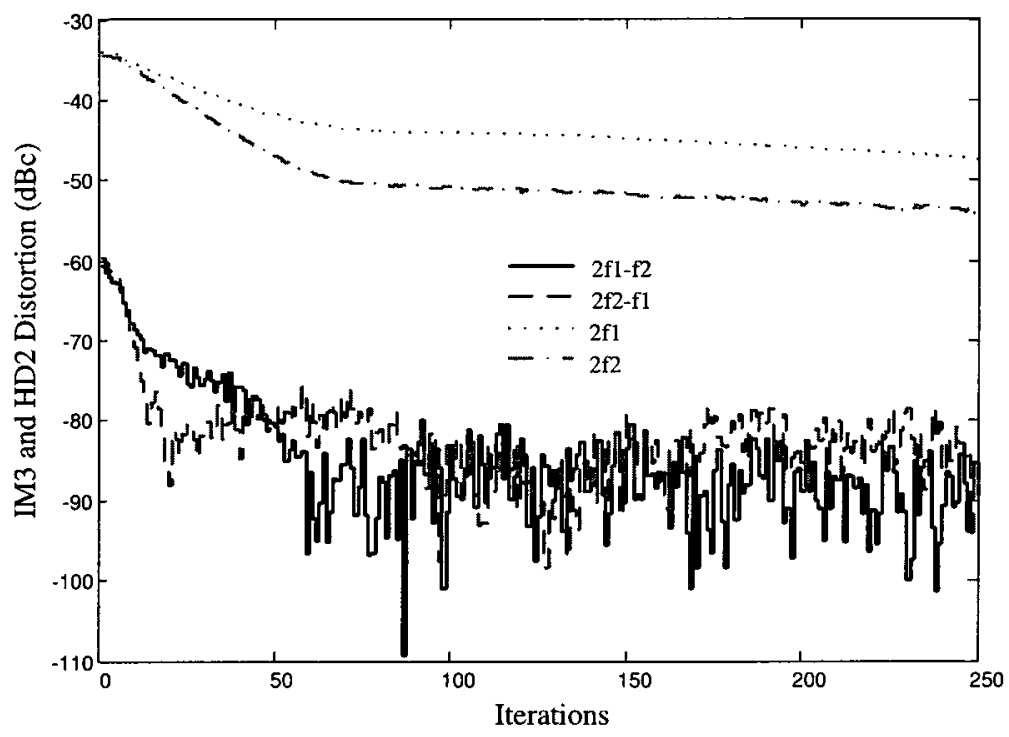

(b)

Figure 6.18: Measured results showing the calibration process with $\alpha_{1,2,3,4}=0.06$ and $\alpha_{5,6,7,8}=0.02$. 


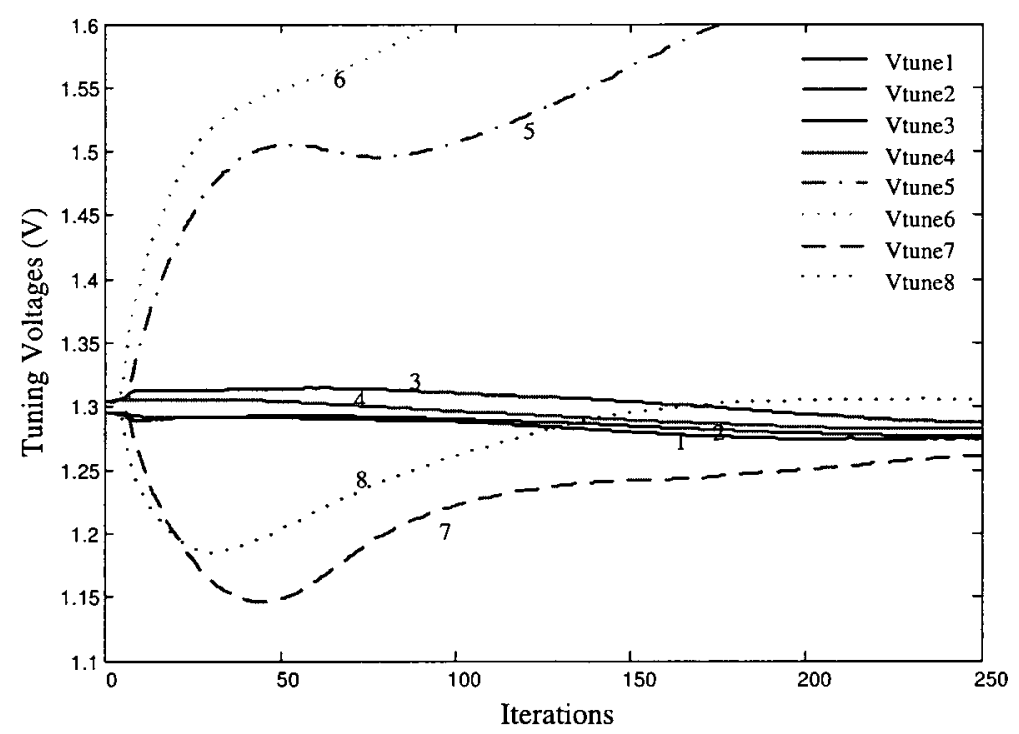

(a)

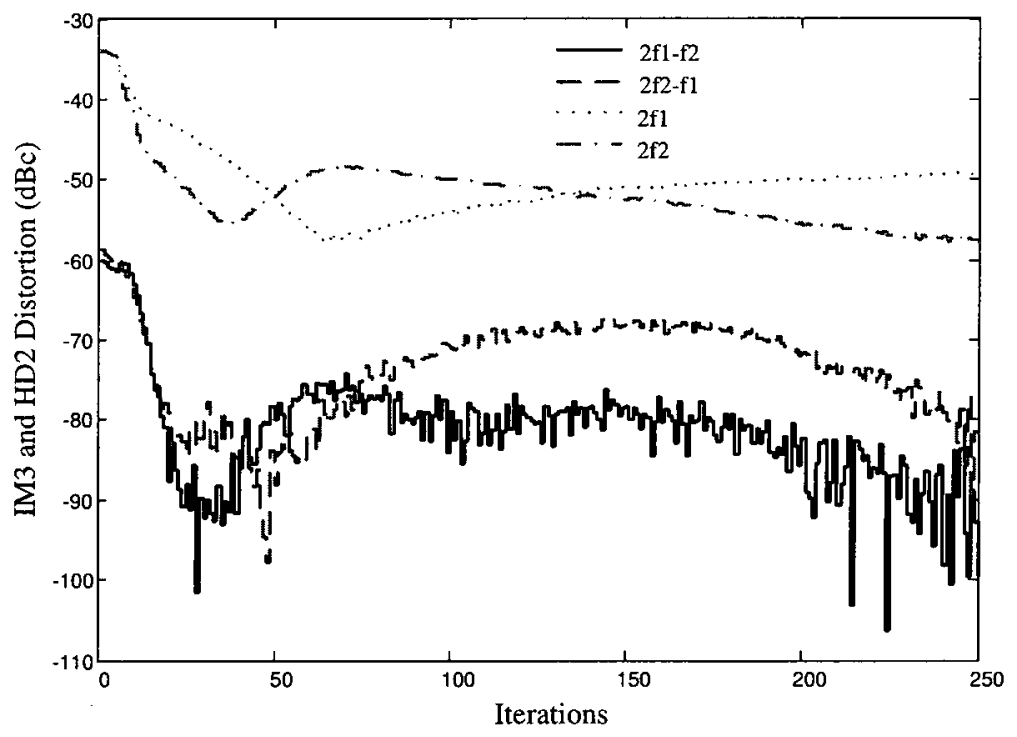

(b)

Figure 6.19: Measured results showing the calibration process with $\alpha_{1,2,3,4}=0.06$ and $\alpha_{5,6,7,8}=0.06$. 
The laser model is implemented in the MatLab software environment. We apply variations to the laser bias current to obtain the behavioral variations of the laser. This works as a substitute in the test plan for the behavioral variation of the laser due to temperature change and device aging.

To test this tracking capability, during the measurement, the laser bias current was purposely changed. It was first increased by $2 \mathrm{~mA}$. Then, after the calibration process settled, the bias current was then decrease by $2 \mathrm{~mA}$. The measured results are shown in Figure 6.20. It is demonstrated that the calibration is able to tracks to a low distortion level in about 30 feedback iterations for IM3 distortion.

It is observed that the IM3 distortion goes up gain. This is due to the K-matrix mismatch discussed in the previous section. The settling of the HD2 distortion influences the settling of the IM3 distortion. If we observe the settling for a longer time, the IM3 distortion will eventually settles down to the low distortion level as shown in Figure 6.21

This tracking test also demonstrates that the feedback algorithm is robust, and does not require a very accurate $\mathrm{K}$ matrix since the $\mathrm{K}$ matrix used in the feedback is estimated at the starting points and it would not exactly match to the laser with a different bias current. 


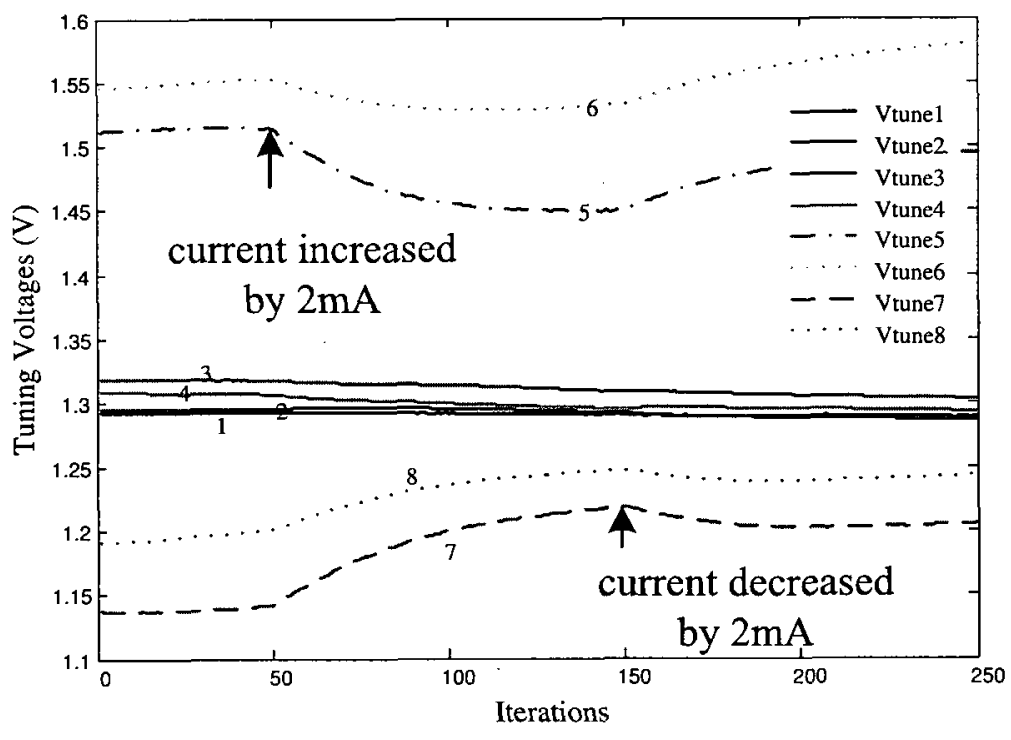

(a)

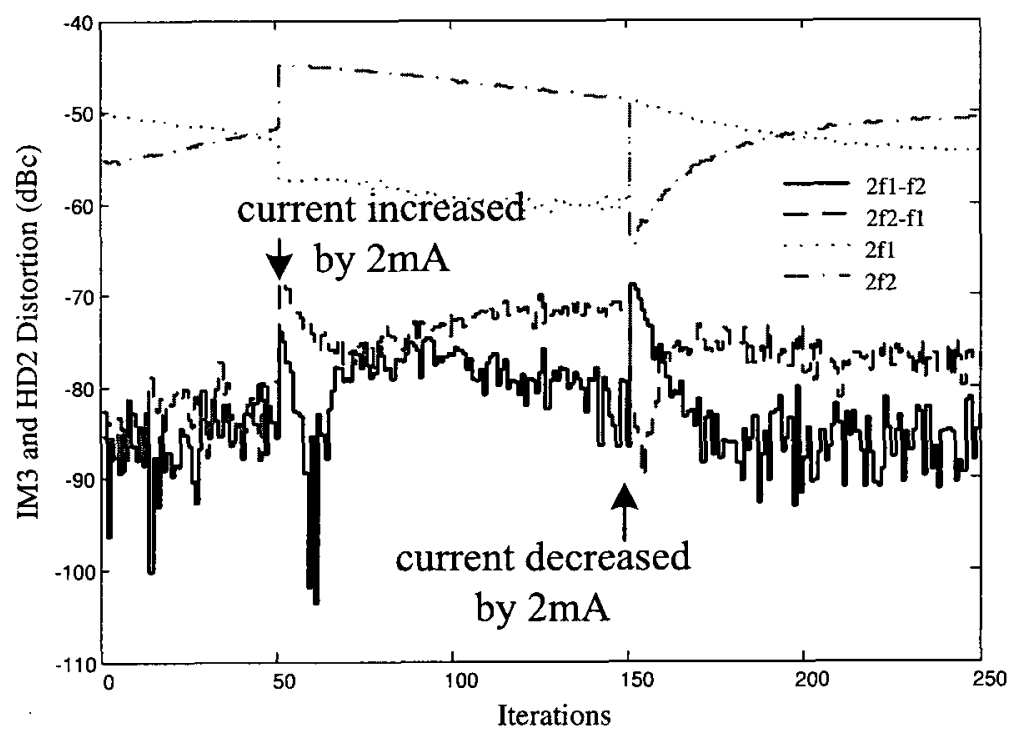

(b)

Figure 6.20: Measured results showing the capability of tracking to the change of bias current. 


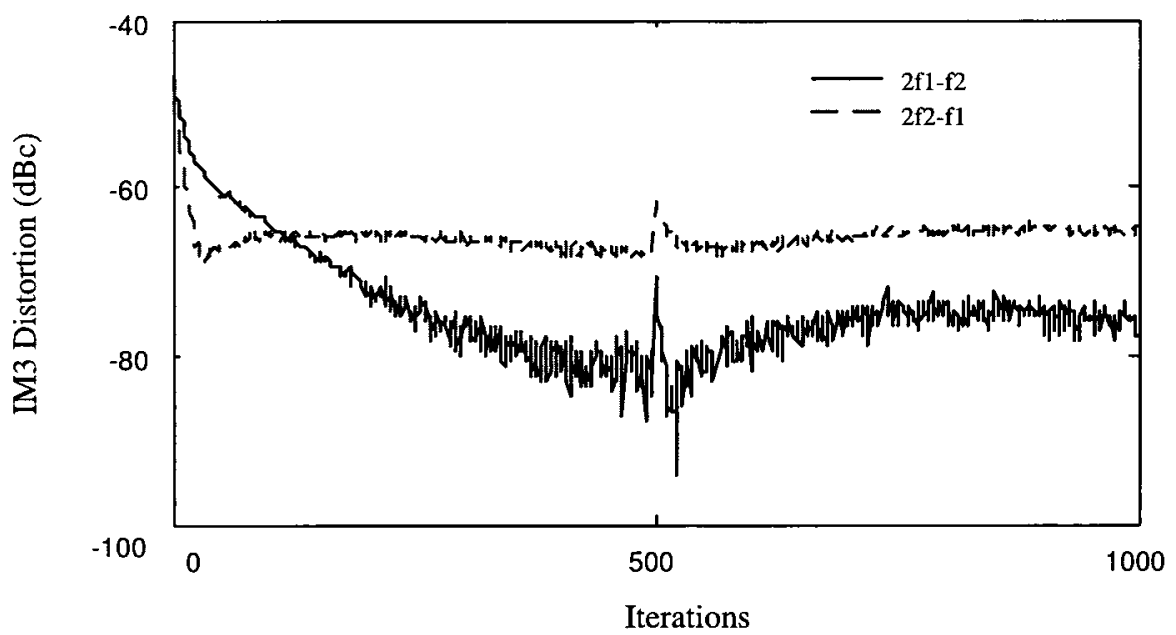

(a)

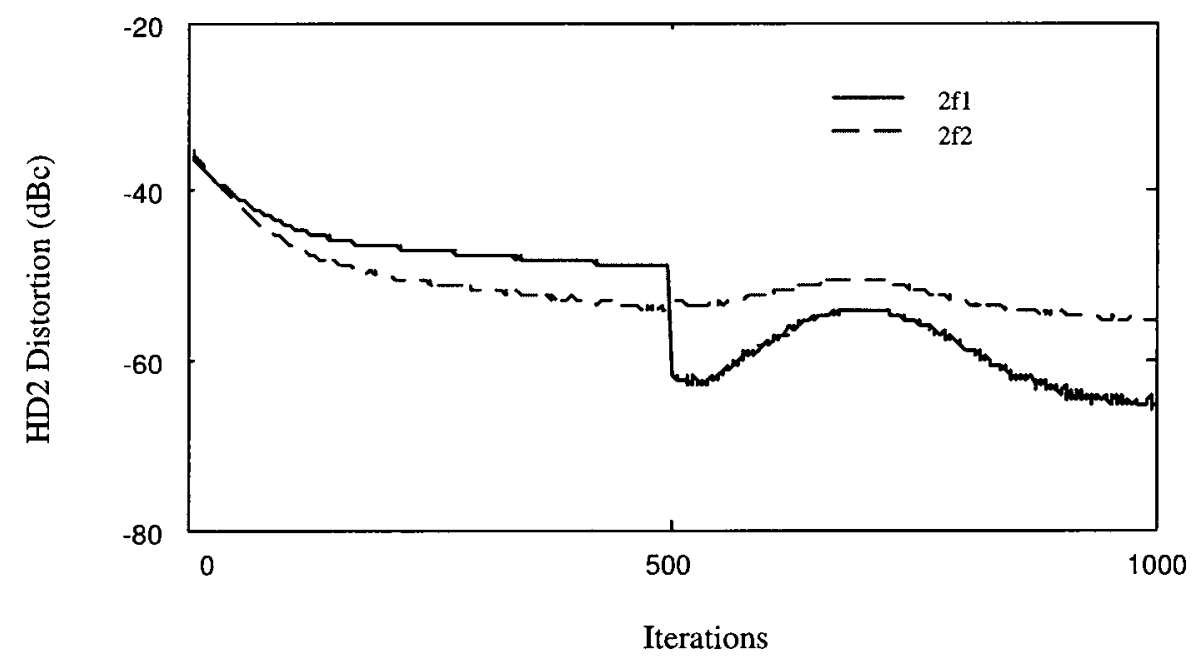

(b)

Figure 6.21: Long term observation of the settling after tracking for (a) IM3 distortion and (b) HD2 distortion. 


\section{Frequency and Power Sweep}

Figure 6.22 shows the HD2 and IM3 curves versus frequency of the predistortion-compensated laser compared with those of the uncompensated laser. A 5-15 dB reduction of IM3 distortion can be observed from $1.8 \mathrm{GHz}$ to $2.2 \mathrm{GHz}$ and a $5-15 \mathrm{~dB}$ reduction of $\mathrm{HD} 2$ distortion can be observed from $1.95 \mathrm{GHz}$ to $2.2 \mathrm{GHz}$. It can be seen that the linearized region for the HD2 distortion is shifted to a higher frequency. This is because the frequency responses of the tank circuits are shifted due to the couplings between the inductors.

The HD2 and IM3 component versus output optical intensity are shown in Figure 6.23. The improvement region for IM3 is between $-10 \mathrm{dBm}$ to $-7 \mathrm{dBm}$ optical intensity, which corresponds to a $6 \mathrm{~dB}$ increase of the output swing for IM3 distortion less than $-67 \mathrm{dBc}$. However, compared with the simulation results shown in Figure 4.24 of Chapter 4 , the measurement results are worse. During the measurement, we observed that, if the tuning voltages were set to $1.3 \mathrm{~V}$ which was supposed to let the predistorter "quiet", there were still some outputs detected from the predistorter. These outputs were probably some even-mode signals passing through the nonideal balun. The predistorter tends to compensate these undesired outputs, thereby degrading its capability to compensate the laser distortion. Applying circuit design techniques to increase the common-mode rejection and layout techniques to maximize the symmetry of the circuit might help to improve the performance.

To further verify the functionality of the predistorter, the IM2 distortion at $\left(f_{1}+f_{2}\right)$ was measured and the results are shown in Figure 6.24. It can be observed that IM2 distortion is reduced by $5-15 \mathrm{~dB}$ from $1.9 \mathrm{GHz}$ to $2.2 \mathrm{GHz}$. Even though the IM2 component at $\left(f_{1}+f_{2}\right)$ is not used in the calibration, it also get improved as the IM2 component resides in the interpolated HD2 band. This IM2 distortion reduction also exhibits the same problem as the HD2 reduction that the linearized region is shifted to higher frequency. 


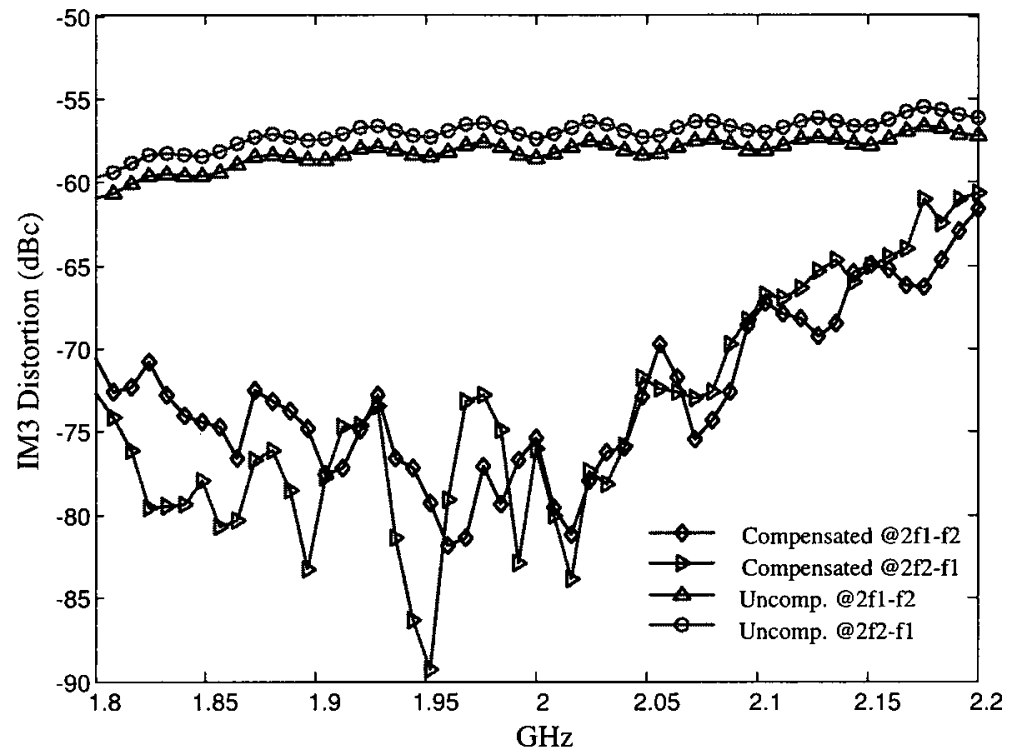

(a)

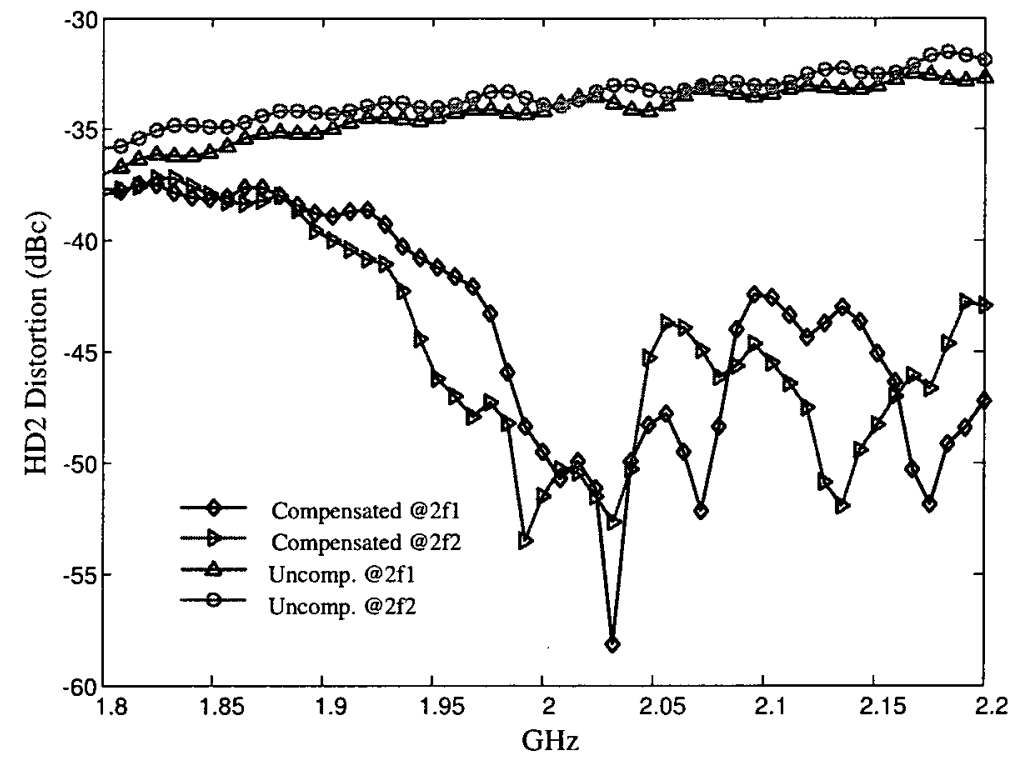

(b)

Figure 6.22: IM3 and HD2 distortion versus frequency. 


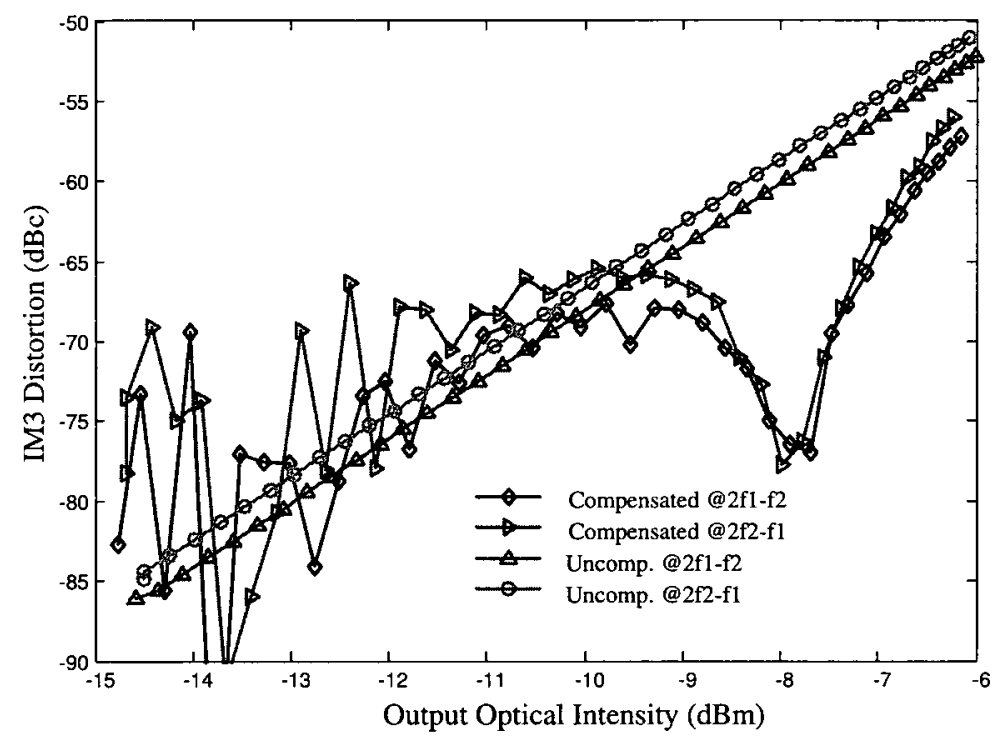

(a)

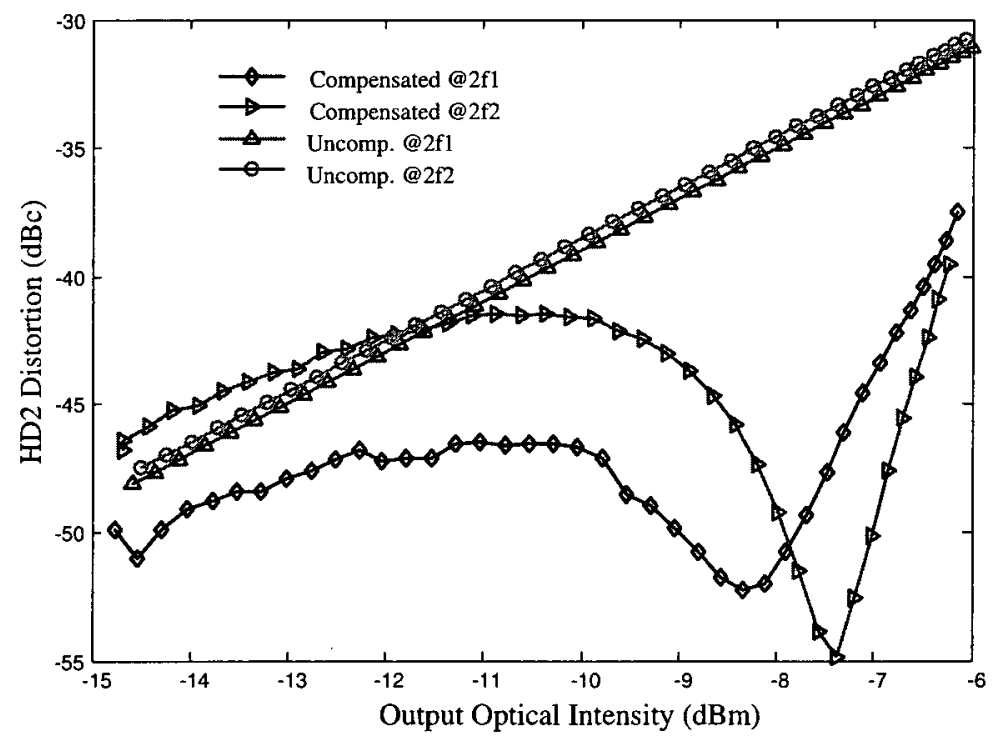

(b)

Figure 6.23: IM3 and HD2 distortion versus output optical intensity. 


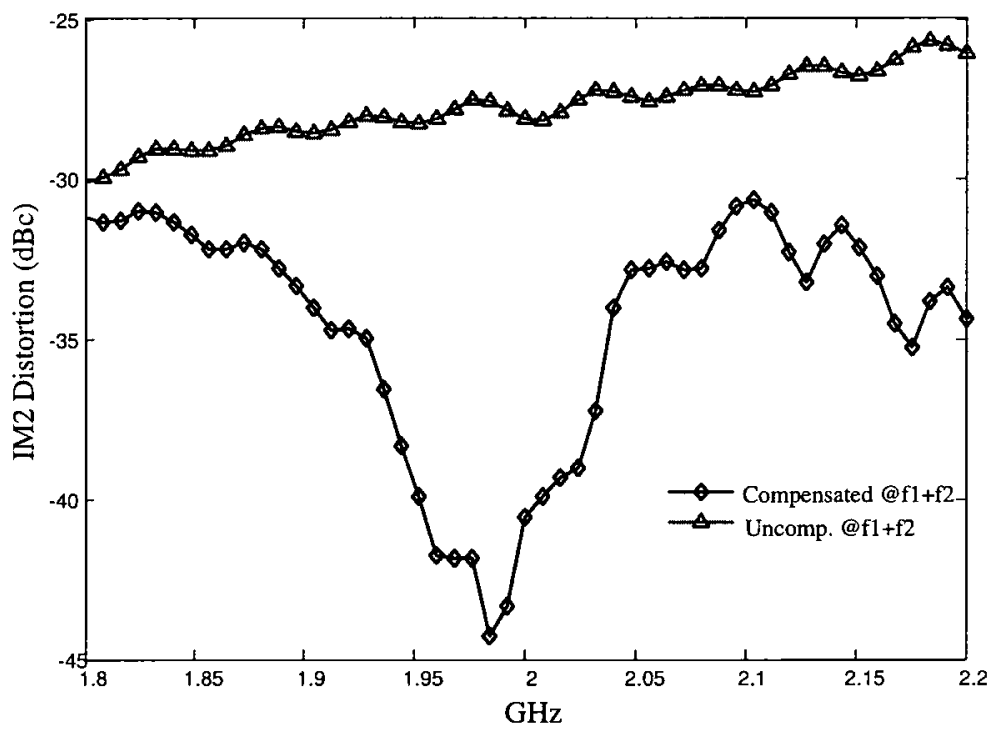

(a)

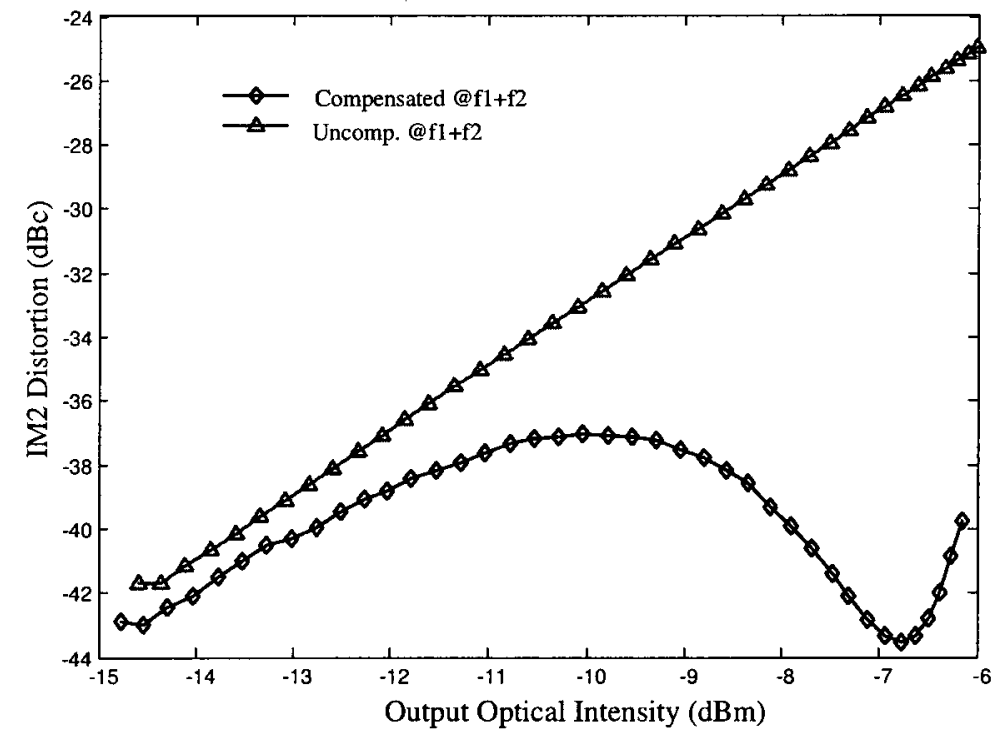

(b)

Figure 6.24: IM2 distortion measurement results. 


\subsection{Summary}

In this chapter, two methods for calibration were briefly discussed. One method is based on optimization and the other is based on iteration (feedback). While the first method has to be realized by digital circuits due to the needs for memories, the second method provides the possibility of an analog implementation.

The fabricated prototype predistorter was put to test in this chapter. The IC layout and test board design were discussed briefly. Test setup were explained in this chapter. An adaptive predistortion calibration technique based on iteration was presented. Applying the proposed calibration technique, the measurement results of the predistorter IC were extracted. It was demonstrated that the calibration control is capable of tracking the variations of the laser bias current. The measurement results demonstrated the wideband linearization performance of the predistorter, which justifies the validity of the proposed multiple tank predistortion circuit design technique. Certain problems of the designed prototype predistorter were discussed and potential solutions were suggested. 


\section{Chapter 7}

\section{Conclusions}

\subsection{Conclusions}

In this thesis, a laser predistorter design technique has been proposed. The technique uses multiple tank circuits to approximate the frequency profile shaping filters in the band of interest. The tank circuit in each tunable gain cell is driven by a Gilbert cell, where the tuning voltage is used to adjust the gain of each cell monotonically. By using multiple tank circuits and adjusting the gain of each cell, both phase and amplitude can be matched to the shaping filter for a wide bandwidth, to achieve wideband linearization. This technique has several desirable features: by working only at the band of interest, the gain-bandwidth trade-off is greatly relieved; it provides a simple access to control the phase and amplitude of the frequency profile. Taken together, these make it possible to design a integrated predistortion circuit.

This thesis, in particular, introduced a complete design procedure which formalized each design step for the proposed multi-tank predistorter. This thesis also proposed an adaptive calibration technique applied to the multi-tank predistortion circuit, by using a multi-variable feedback algorithm. Compared with the available adaptive predistortion techniques in $[50,58]$, this technique directly processes the RF signal and does not require digital signal processing but instead uses analog feedback to perform the calibration. It suggests a potential complete analog implementation with less circuit complexity.

For the purpose of demonstration, a prototype CMOS predistorter IC has been designed 
as an example, which works at $2 \mathrm{GHz}$ and has a $300 \mathrm{MHz}$ linearization bandwidth. The maximum output optical power is $-7 \mathrm{dBm}$. $5-15 \mathrm{~dB}$ reduction of the $2^{\text {nd }}$ harmonic distortion and $3^{\text {rd }}$ order intermodulation distortion can be obtained in the measurement. In terms of bandwidth, the predistortion circuit is able to work for most of the wireless standards, such as GSM, CDMA and WiMax.

Even though a narrow band example is given in this thesis, the multi-tank technique does not prevent much wider bandwidth, which can be extended by using more tank circuits to match the shaping curve for a wider range. The price to pay is to have more tuning terminals, higher power consumption, and greater complexity.

\subsection{Recommendations for Future Research}

The following list provides the ideas of continuing the research on laser predistortion:

- The predistorter circuit should be redesigned using circuit techniques to enhance the common-mode rejection. Layout techniques, such as common-centroid and interdigitated layout, should be applied to maximize the symmetry of the differential circuit, therefore, reducing the even-mode signal's leakage to the laser.

- Linear path time delay circuit implementation. In the current prototype design, one piece of coaxial cable was used to provide the required time delay. It is quite long and not suited for a practical usage. Using bandpass LC-ladder filter [80] to achieve the time delay function is a good option, as it preserves the linearity of passive networks and can be realized on the chip. Another option is to place a laser driver in the linear path for providing both gain and time delay for the linear path. The predistorter should be able to linearize both the laser driver and the laser. However, depending on the nonlinearity of the laser driver, the capability of linearizing the laser will be reduced.

- Miniaturization of the wideband balun. The wideband balun proposed in this thesis was realized on PCB. Its size is quite large. For miniaturization, there are many examples of implementing Marchand baluns on LTCC [81] and MMIC [71,82]. While 
the required balun is full-matched and wideband, it seems promising to obtain such a balun on LTCC or MMIC substrate if applying the design concept proposed in Chapter 5.

- Advanced loop filter design. Simple accumulators were used as loop filters for the calibration algorithm in this thesis, the convergence speed is quite slow due to the narrow loop bandwidth. Conventional loop filter design techniques for Phase Lock Loop (PLL) can be applied to achieve a fast settling of the calibration process.

- Hardware implementation of the feedback control algorithm. Figure 7.1 shows the possible circuit implementation of the feedback control algorithm. The frequency synthesizer is used to generate tones as HD2 and IM3 frequencies one at a time. The output of photodiode is amplified by the Transimpedance Amplifier (TIA) then multiplied with the output of the frequency synthesizer by a mixer. Then the real or imaginary part of the laser distortion can be extracted at DC by a lowpass filter. Depending on the output frequency of the synthesizer at the time, the output of the lowpass filter connects to loop1, loop $2, \ldots$ or loop 8 . The $\mathrm{K}$ matrix is realized by a group of operational amplifiers, where the resistors are used to define the elements of the $\mathrm{K}$ matrix. The outputs of the $\mathrm{K}$ matrix are the tuning voltages for the predistorter. For a predistorter using digitally controlled gain cell (see an example in Figure 4.10), an Analog to Digital Converter (ADC) is required. All the control loops can share the same ADC and use it one at a time alternatively. The registers will hold the outputs of the ADC to adjust the tunable gain cell. A potential problem of using one ADC is that the loop filters need to hold the analog values for a longer time and the current leakage of active device may reduce the loop gain significantly. In this case, each loop can have its own ADC to solve the problem. High performance and complicated ADCs are not necessary, simple ADC structures, such as Hopfield ADC [83], can be used to reduce the circuit complexity and save the silicon area.

- Fully integrated laser predistortion system. With all the components described above designed, the next task to accomplish is definitely to make a fully integrated laser 


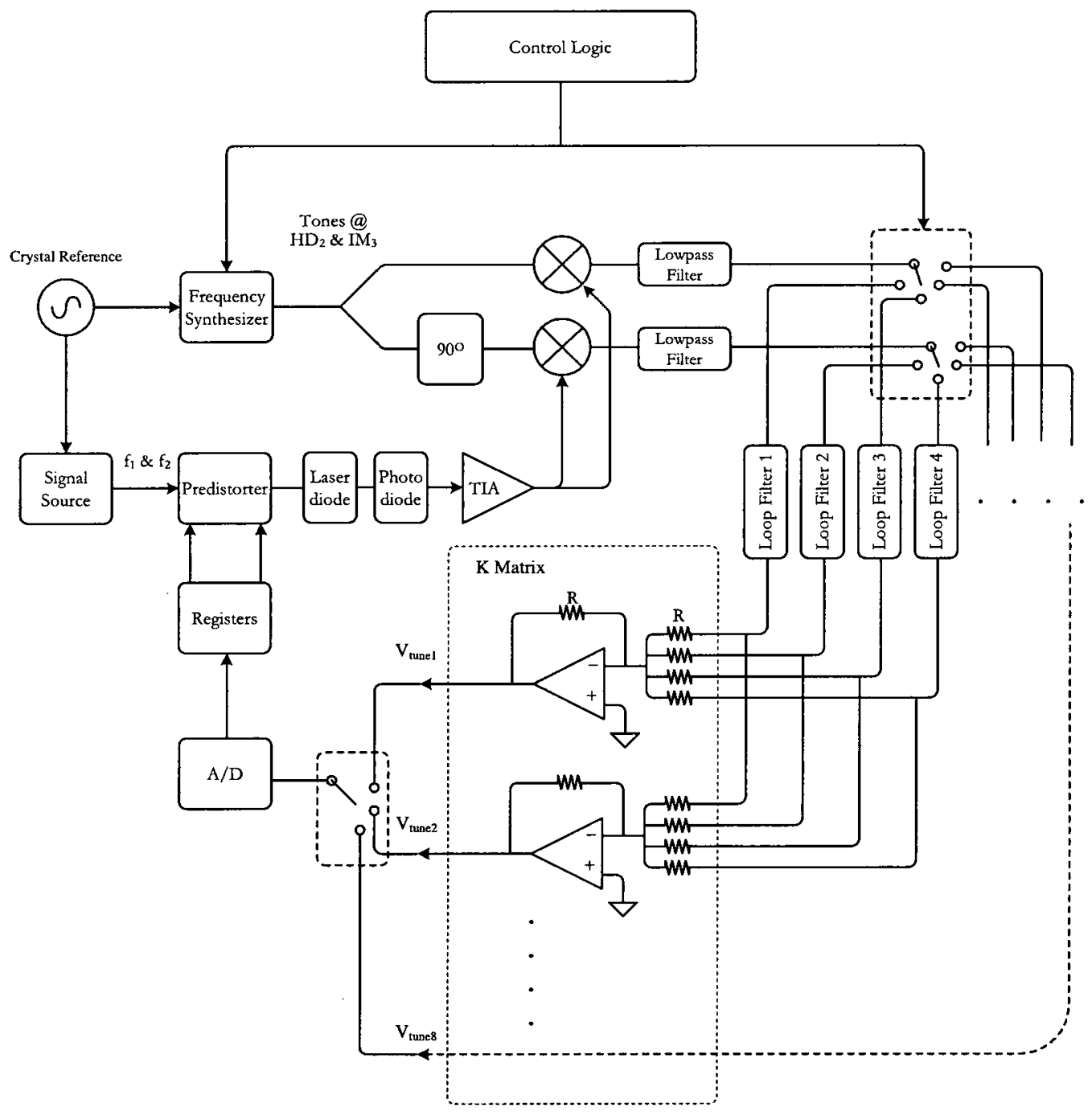

Figure 7.1: Possible circuit implementation of the feedback control algorithm. 
predistortion system.

- Applying the multi-tank circuit design technique and the multiple variable feedback technique to linearize other circuits, such as power amplifiers. The predistortion technique proposed in this thesis is a general purpose predistortion scheme, it should be able to linearize other circuits.

- Using more tank circuits in the design for a much larger linearization bandwidth. Multiple pilot tones might be required in order for calibrating the predistortion circuit. 


\section{Appendix A}

\section{Laser Inverse Model Constants}

The coefficients $m_{0} \ldots m_{8}$ in the laser inverse model are given by

$$
\begin{aligned}
m_{0}= & -C_{t r} g_{m} M_{0} q V_{c}-C_{t r} g_{m} M_{1} P_{d c} q V_{c}+g_{m} M_{0} q V_{c} Z_{0}+g_{m} M_{1} P_{d c} q V_{c} Z_{0} \\
& +g_{m} M_{0} P_{d c} q V_{c} Z_{1}+\frac{q V_{c} Z_{1}}{\tau_{e}} \\
m_{1}= & q V_{c} Z_{1}+g_{m} M_{0} P_{d c} q V_{c} Z_{2}+\frac{q V_{c} Z_{2}}{\tau_{e}} \\
m_{2}= & q V_{c} Z_{2} \\
m_{3}= & -C_{t r} g_{m} M_{1} q V_{c}-C_{t r} g_{m} M_{2} P_{d c} q V_{c}+g_{m} M_{1} q V_{c} Z_{0}+g_{m} M_{2} P_{d c} q V_{c} Z_{0} \\
& +g_{m} M_{0} q V_{c} Z_{1}+g_{m} M_{1} P_{d c} q V_{c} Z_{1}+g_{m} M_{0} P_{d c} q V_{c} Z_{3}+\frac{q V_{c} Z_{3}}{\tau_{e}} \\
m_{4}= & \left(g_{m} M_{0} q V_{c} Z_{2}+g_{m} M_{1} P_{d c} q V_{c} Z_{2}+2 q V_{c} Z_{3}+g_{m} M_{0} P_{d c} q V_{c} Z_{4}+\frac{q V_{c} Z_{4}}{\tau_{e}}\right) / 2 \\
m_{5}= & q V_{c} Z_{4} / 2 \\
m_{6}= & -C_{t r} g_{m} M_{2} q V_{c}-C_{t r} g_{m} M_{3} P_{d c} q V_{c}+g_{m} M_{2} q V_{c} Z_{0} \\
& +g_{m} M_{3} P_{d c} q V_{c} Z_{0}+g_{m} M_{1} q V_{c} Z_{1}+g_{m} M_{2} P_{d c} q V_{c} Z_{1} \\
& +g_{m} M_{0} q V_{c} Z_{3}+g_{m} M_{1} P_{d c} q V_{c} Z_{3} \\
& +g_{m} M_{0} P_{d c} q V_{c} Z_{5}+\frac{q V_{c} Z_{5}}{\tau_{e}} \\
m_{7}= & \left(g_{m} M_{1} q V_{c} Z_{2}+g_{m} M_{2} P_{d c} q V_{c} Z_{2}\right) \\
& +g_{m} M_{0} q V_{c} Z_{4}+g_{m} M_{1} P_{d c} q V_{c} Z_{4}+3 q V_{c} Z_{5} \\
& \left.+g_{m} M_{0} P_{d c} q V_{c} Z_{6}+\frac{q V_{c} Z_{6}}{\tau_{e}}\right) / 3 \\
m_{8}= & q V_{c} Z_{6} / 3 \\
g_{m}= & v_{g} g
\end{aligned}
$$


where

$$
\begin{aligned}
Z_{0}= & C_{t r} D_{0} G M_{0} P_{d c}+\frac{D_{0} P_{d c}}{\tau_{p h}} \\
Z_{1}= & C_{t r} D_{0} G M_{0}+C_{t r} D_{1} G M_{0} P_{d c}+C_{t r} D_{0} G M_{1} P_{d c}+\frac{D_{0}}{\tau_{p h}}+\frac{D_{1} P_{d c}}{\tau_{p h}} \\
Z_{2}= & D_{0} \\
Z_{3}= & C_{t r} D_{1} G M_{0}+C_{t r} D_{0} G M_{1}+C_{t r} D_{2} G M_{0} P_{d c}+C_{t r} D_{1} G M_{1} P_{d c} \\
& +C_{t r} D_{0} G M_{2} P_{d c}+\frac{D_{1}}{\tau_{p h}}+\frac{D_{2} P_{d c}}{\tau_{p h}} \\
Z_{4}= & D_{1} \\
Z_{5}= & C_{t r} D_{2} G M_{0}+C_{t r} D_{1} G M_{1}+C_{t r} D_{0} G M_{2}+C_{t r} D_{3} G M_{0} P_{d c} \\
& +C_{t r} D_{2} G M_{1} P_{d c}+C_{t r} D_{1} G M_{2} P_{d c}+C_{t r} D_{0} G M_{3} P_{d c}+\frac{D_{2}}{\tau_{p h}}+\frac{D_{3} P_{d c}}{\tau_{p h}} \\
Z_{6}= & D_{2} \\
Z_{7}= & D_{3} \\
G= & \Gamma v_{g} g
\end{aligned}
$$

and

$$
\begin{aligned}
& D_{0}=\frac{1}{c_{0}+G M_{0} P_{d c}} \\
& D_{1}=\frac{-G\left(M_{0}+M_{1} P_{d c}\right)}{\left(c_{0}+G M_{0} P_{d c}\right)^{2}} \\
& D_{2}=\frac{-G\left(M_{1}+M_{2} P_{d c}\right)}{\left(c_{0}+G M_{0} P_{d c}\right)^{2}}+\frac{G^{2}\left(M_{0}+M_{1} P_{d c}\right)^{2}}{\left(c_{0}+G M_{0} P_{d c}\right)^{3}} \\
& D_{3}=\left(-\frac{\left(G M_{0}+G M_{1} P_{d c}\right)^{3}}{\left(c_{0}+G M_{0} P_{d c}\right)^{4}}+\frac{2\left(G M_{0}+G M_{1} P_{d c}\right)\left(G M_{1}+G M_{2} P_{d c}\right)}{\left(c_{0}+G M_{0} P_{d c}\right)^{3}}-\frac{G M_{2}+G M_{3} P_{d c}}{\left(c_{0}+G M_{0} P_{d c}\right)^{2}}\right) \\
& c_{0}=\Gamma \beta_{s p} / \tau_{e} .
\end{aligned}
$$

At last,

$$
\begin{aligned}
& M_{0}=\frac{1}{\sqrt{1+\delta P_{d c}}} \\
& M_{1}=\frac{-\delta}{2\left(1+\delta P_{d c}\right)^{3 / 2}} \\
& M_{2}=\frac{3 \delta^{2}}{8\left(1+\delta P_{d c}\right)^{5 / 2}} \\
& M_{3}=\frac{-5 \delta^{3}}{16\left(1+\delta P_{d c}\right)^{7 / 2}} .
\end{aligned}
$$


Appendix B

Tank Circuit Optimization Contour Plots 

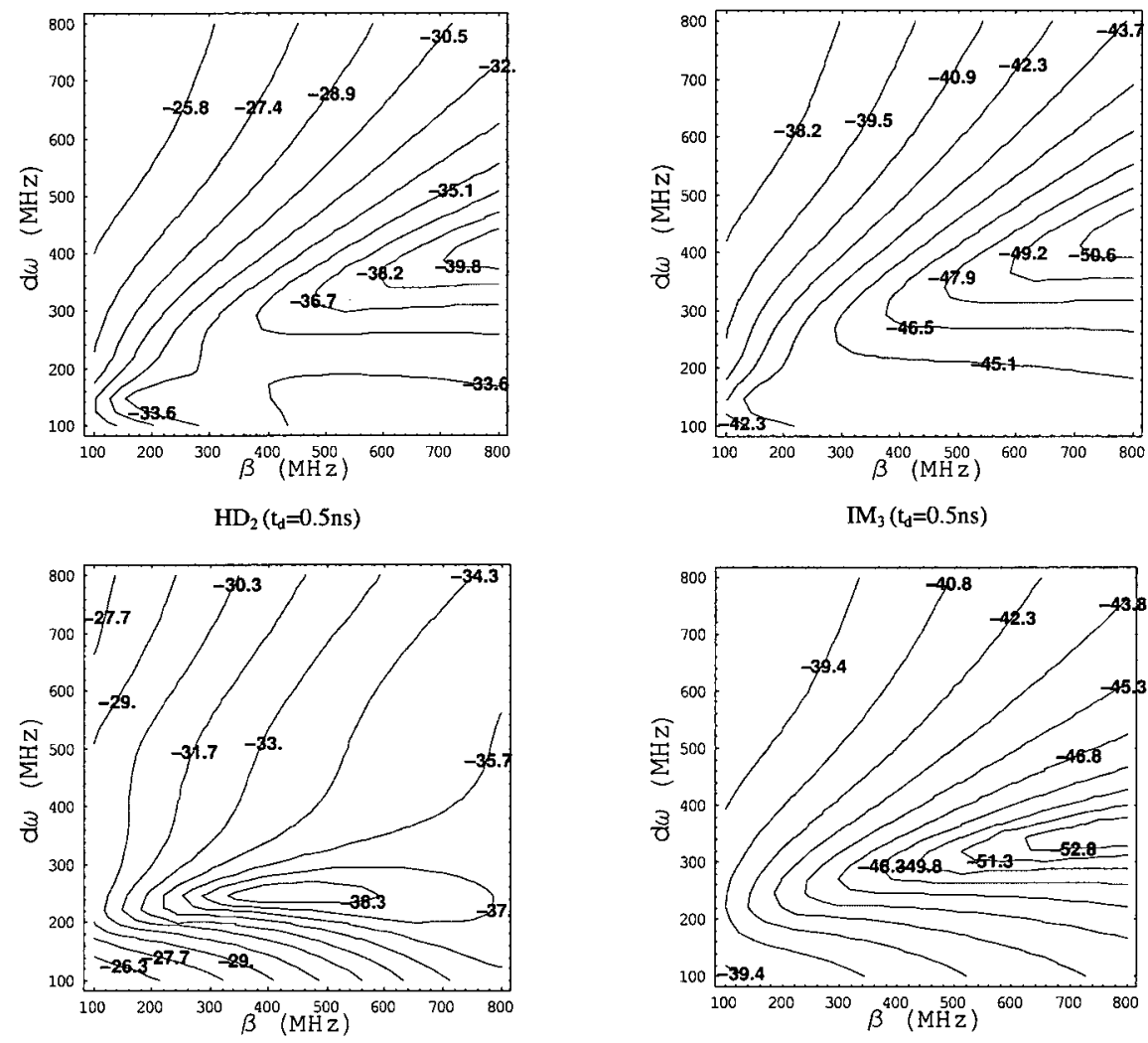

$\mathrm{HD}_{2}\left(\mathrm{t}_{\mathrm{d}}=0.55 \mathrm{~ns}\right)$

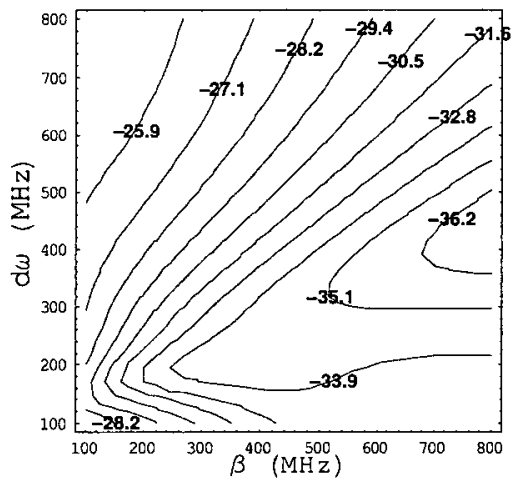

$\mathrm{HD}_{2}\left(\mathrm{t}_{\mathrm{d}}=0.6 \mathrm{~ns}\right)$

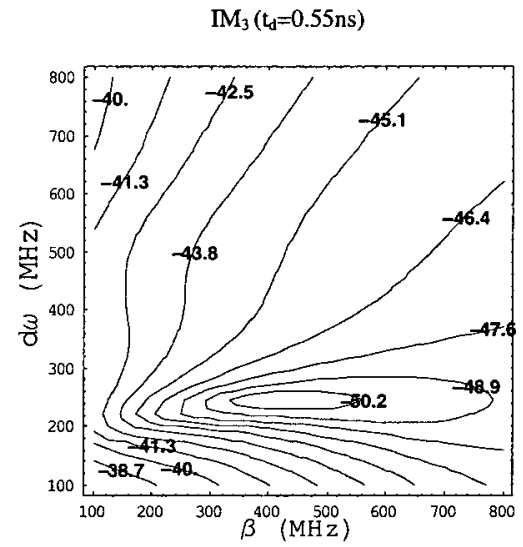

$\mathrm{IM}_{3}\left(\mathrm{t}_{\mathrm{d}}=0.6 \mathrm{~ns}\right)$

Figure B.1: Tank circuit parameters optimization for different linear path delays. 


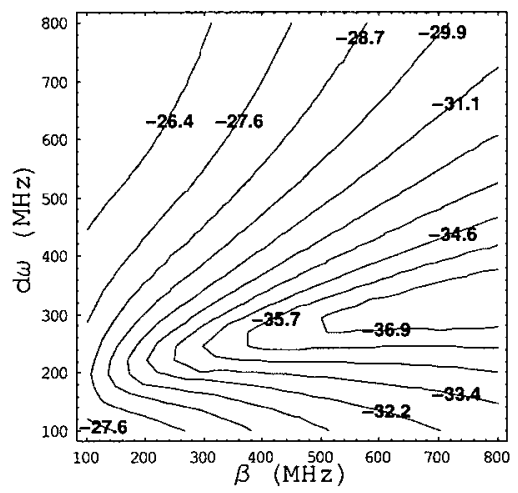

$\mathrm{HD}_{2}\left(\mathrm{t}_{\mathrm{d}}=0.65 \mathrm{~ns}\right)$

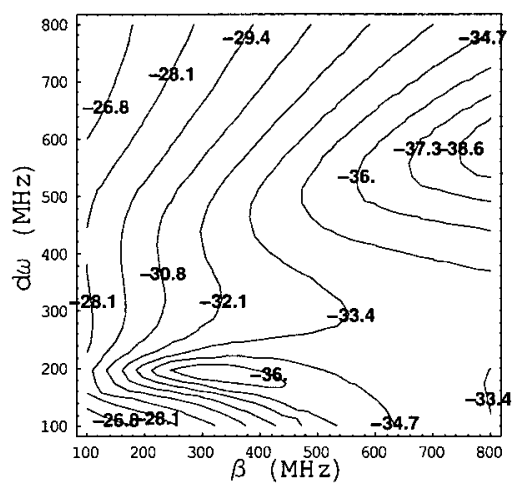

$\mathrm{HD}_{2}\left(\mathrm{t}_{\mathrm{d}}=0.7 \mathrm{~ns}\right)$

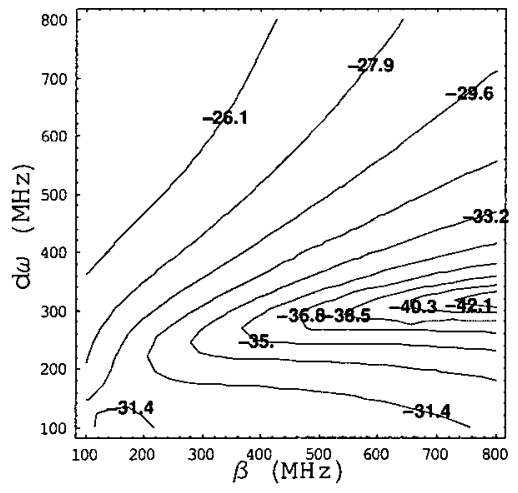

$\mathrm{HD}_{2}\left(\mathrm{t}_{\mathrm{d}}=0.75 \mathrm{~ns}\right)$

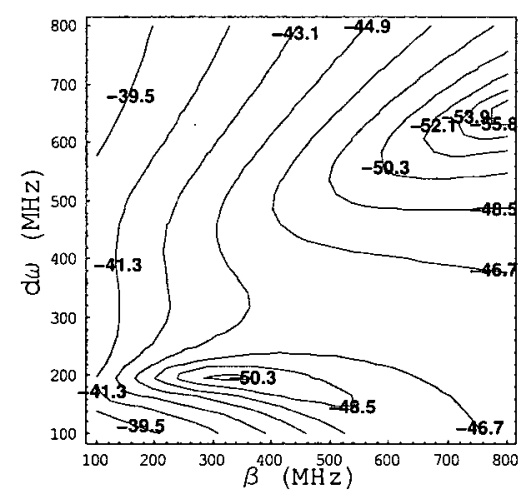

$\mathrm{MM}_{3}\left(\mathrm{t}_{\mathrm{d}}=0.65 \mathrm{~ns}\right)$

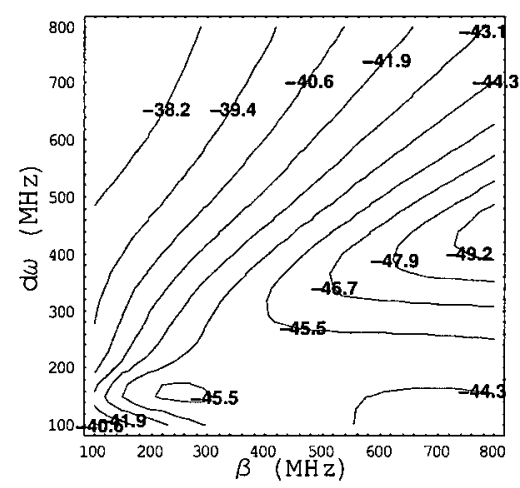

$\mathrm{IM}_{3}\left(\mathrm{t}_{\mathrm{d}}=0.7 \mathrm{~ns}\right)$

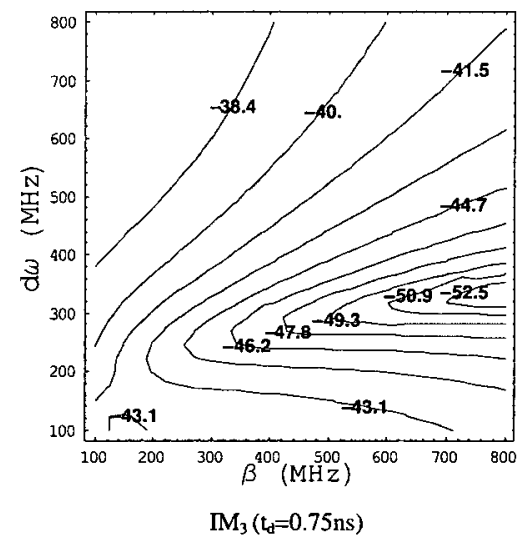

$\mathrm{IM}_{3}\left(\mathrm{c}_{\mathrm{d}}=0.75 \mathrm{~ns}\right)$

Figure B.2: Tank circuit parameters optimization for different linear path delays. 

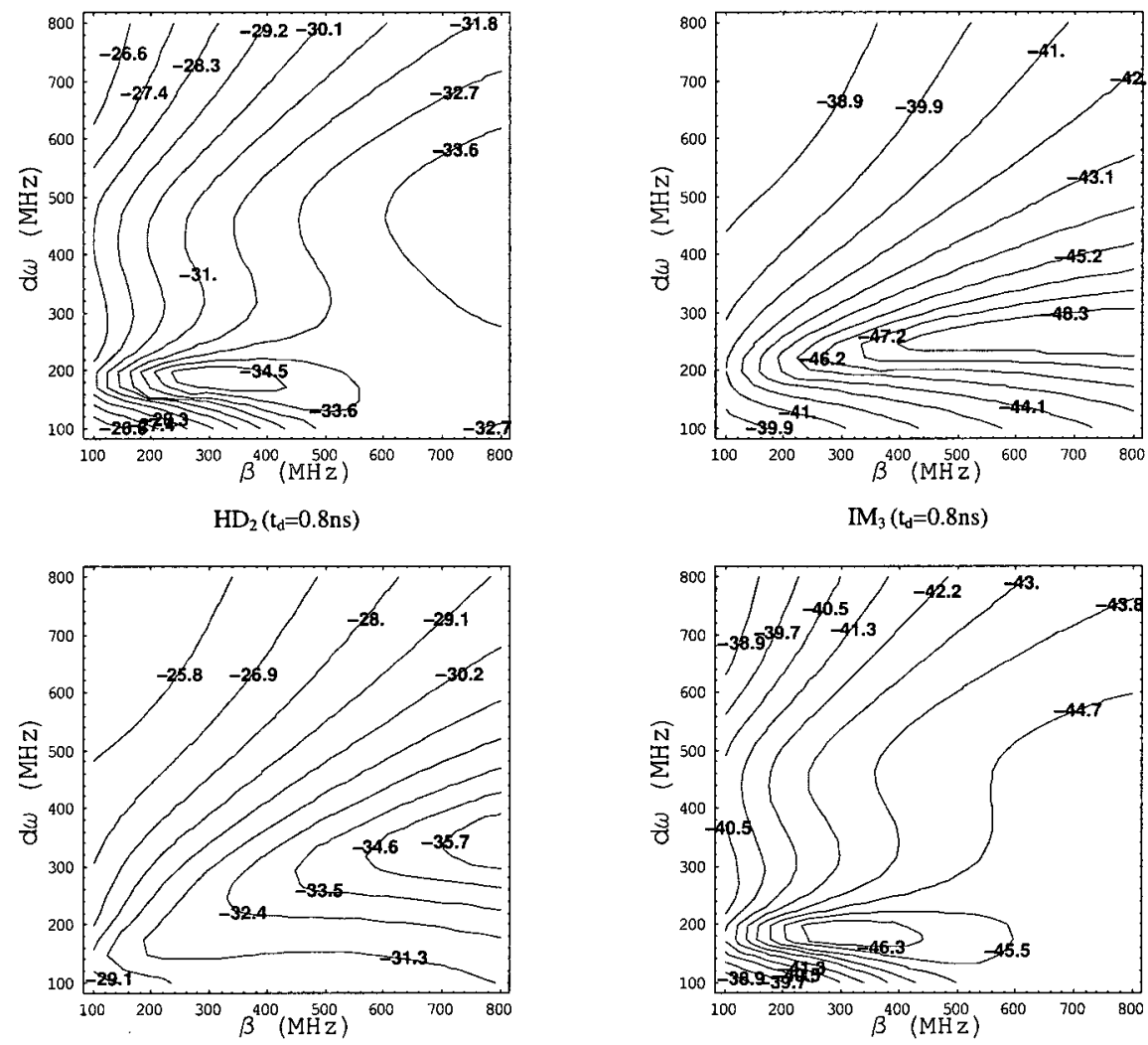

$\mathrm{HD}_{2}\left(\mathrm{t}_{\mathrm{d}}=0.85 \mathrm{~ns}\right)$

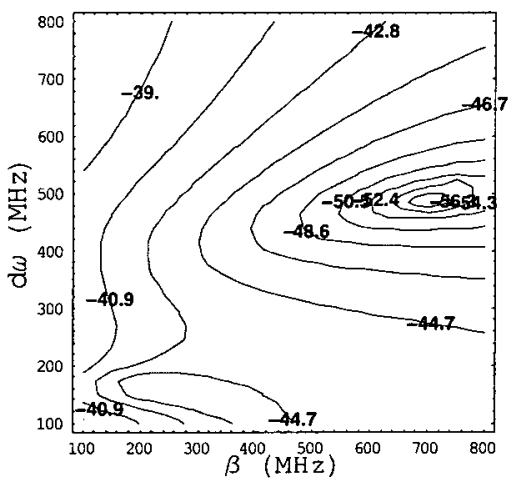

$\mathrm{HD}_{2}\left(\mathrm{t}_{\mathrm{d}}=0.9 \mathrm{~ns}\right)$

$\mathrm{IM}_{3}\left(\mathrm{t}_{\mathrm{d}}=0.9 \mathrm{~ns}\right)$

Figure B.3: Tank circuit parameters optimization for different linear path delays. 


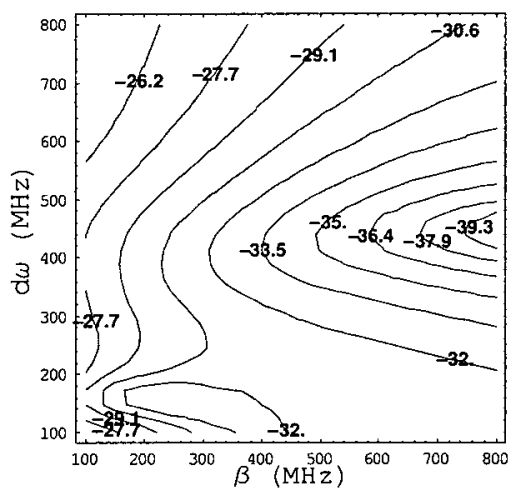

$\mathrm{HD}_{2}\left(\mathrm{t}_{\mathrm{d}}=0.95 \mathrm{~ns}\right)$

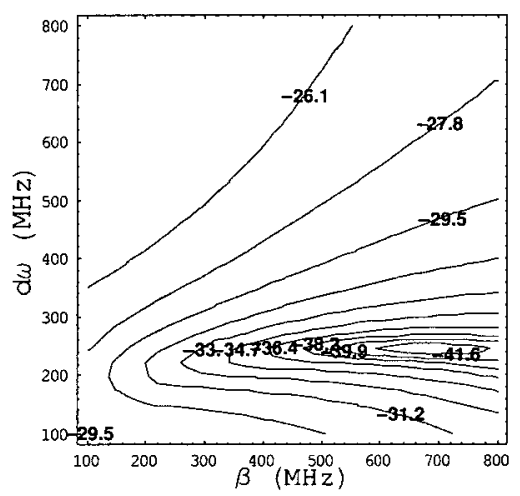

$\mathrm{HD}_{2}\left(\mathrm{t}_{\mathrm{d}}=\operatorname{lns}\right)$

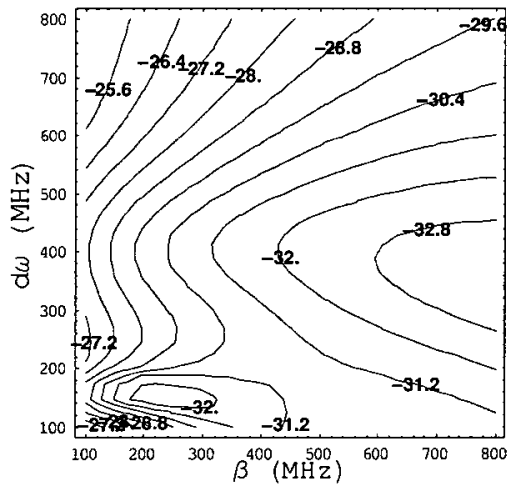

$\mathrm{HD}_{2}\left(\mathrm{t}_{\mathrm{d}}=1.05 \mathrm{~ns}\right)$
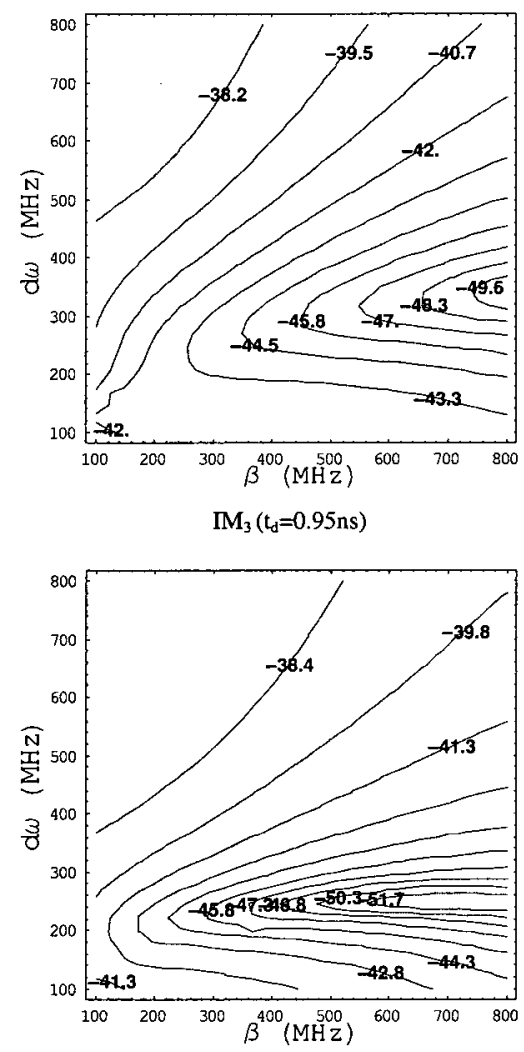

$\mathrm{IM}_{3}\left(\mathrm{t}_{\mathrm{d}}=1 \mathrm{~ns}\right)$

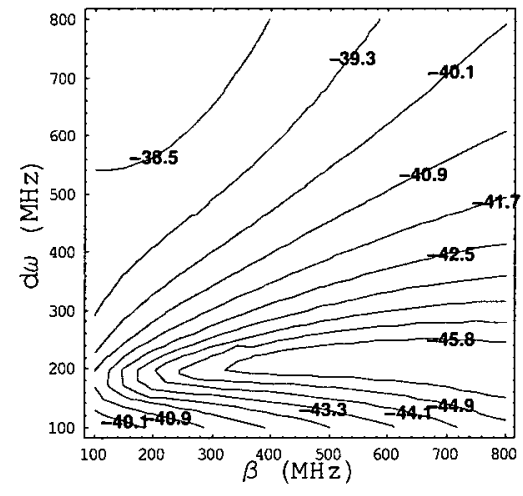

$\mathrm{IM}_{3}\left(\mathrm{t}_{\mathrm{d}}=1.05 \mathrm{~ns}\right)$

Figure B.4: 'Tank circuit parameters optimization for different linear path delays. 

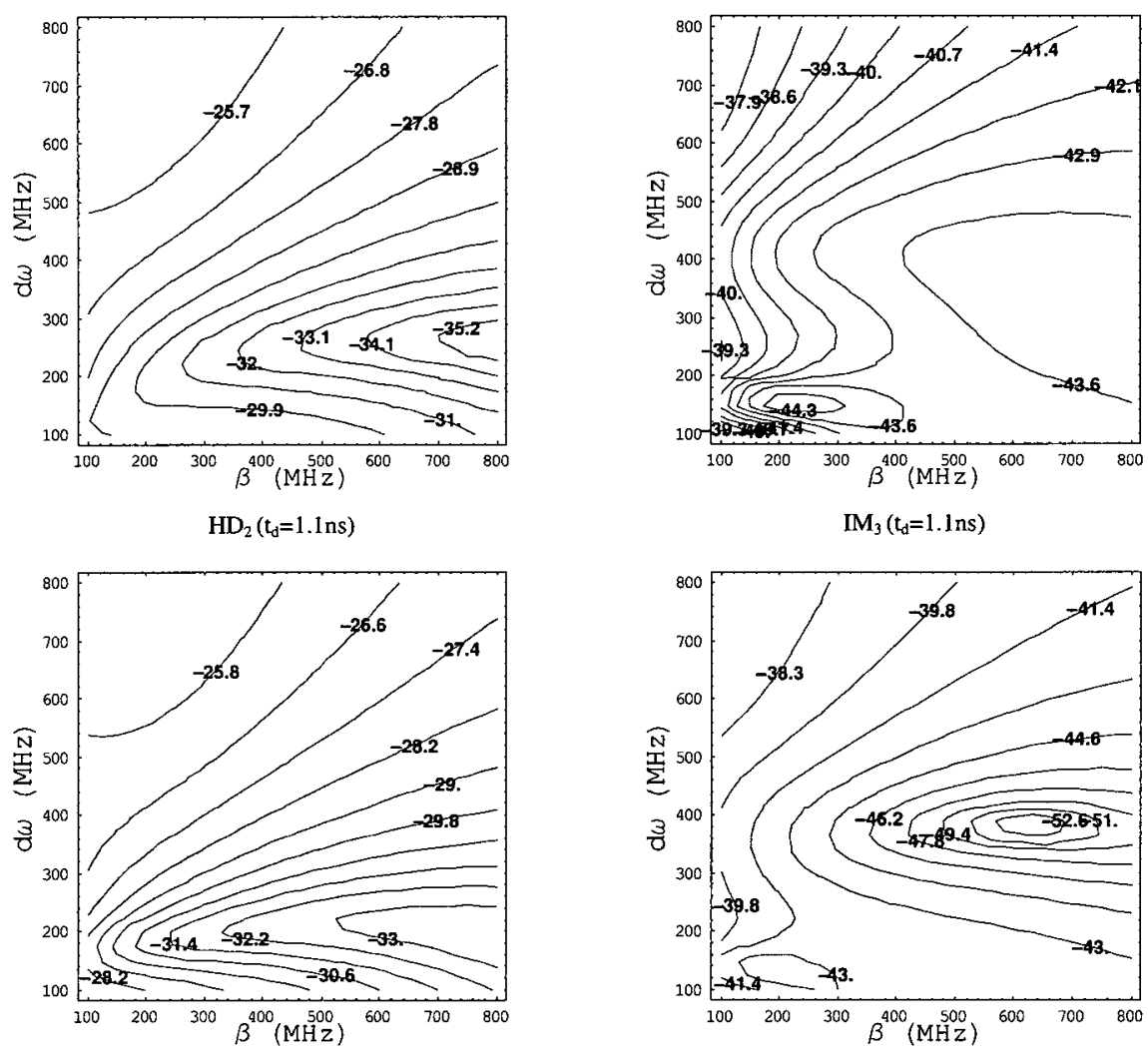

$\mathrm{HD}_{2}\left(\mathrm{t}_{\mathrm{d}}=1.15 \mathrm{~ns}\right)$
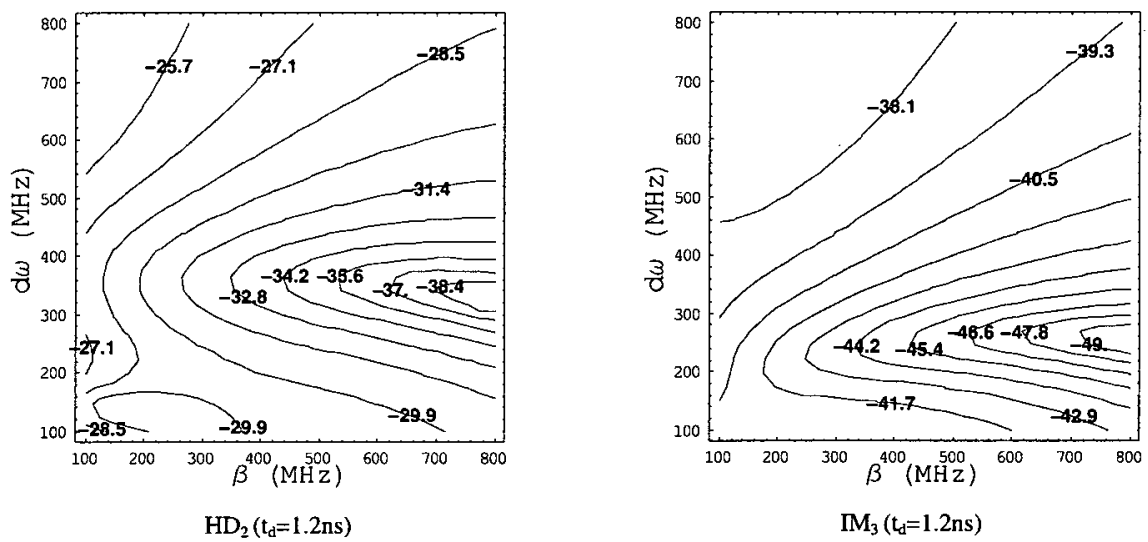

Figure B.5: Tank circuit parameters optimization for different linear path delays. 


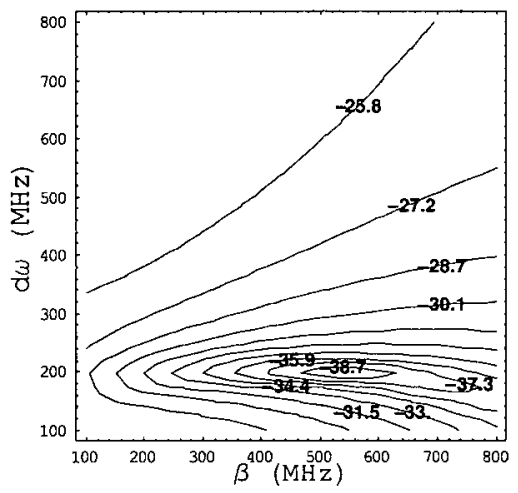

$\mathrm{HD}_{2}\left(\mathrm{t}_{\mathrm{d}}=1.25 \mathrm{~ns}\right)$

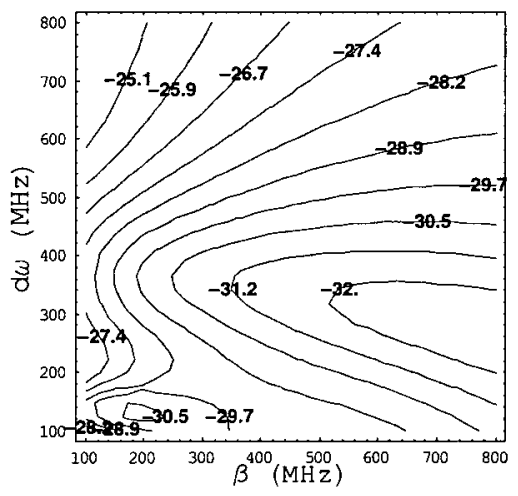

$\mathrm{HD}_{2}\left(\mathrm{t}_{\mathrm{d}}=1.3 \mathrm{~ns}\right)$

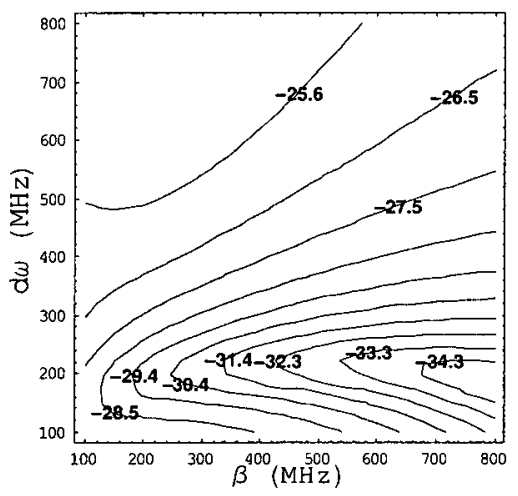

$\mathrm{HD}_{2}\left(\mathrm{t}_{\mathrm{d}}=1.35 \mathrm{~ns}\right)$

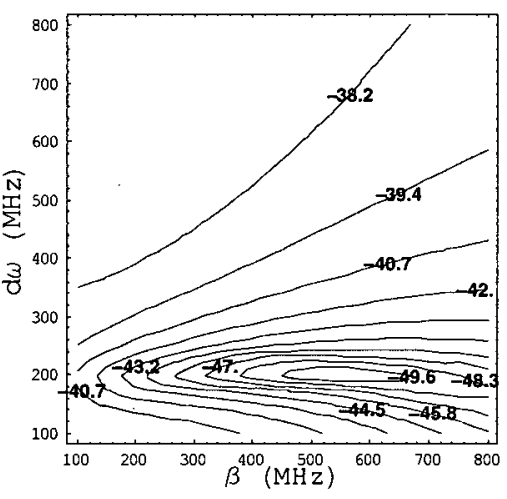

$\mathrm{IM}_{3}\left(\mathrm{c}_{\mathrm{d}}=1.25 \mathrm{~ns}\right)$

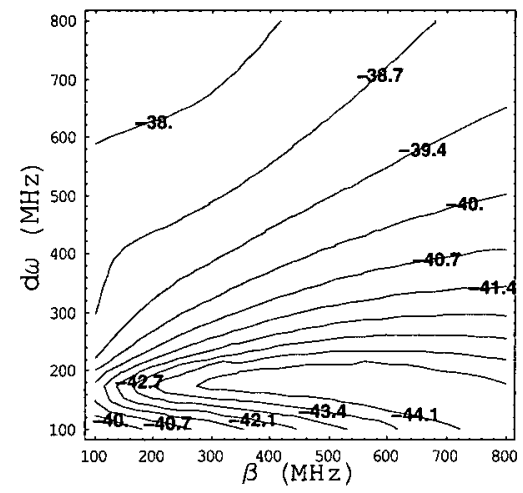

$\mathrm{IM}_{3}\left(\mathrm{t}_{\mathrm{d}}=1.3 \mathrm{~ns}\right)$

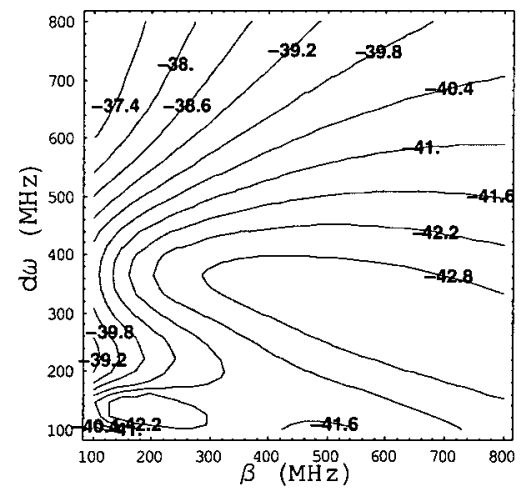

$\mathrm{IM}_{3}\left(\mathrm{t}_{\mathrm{d}}=1.35 \mathrm{~ns}\right)$

Figure B.6: Tank circuit parameters optimization for different linear path delays. 

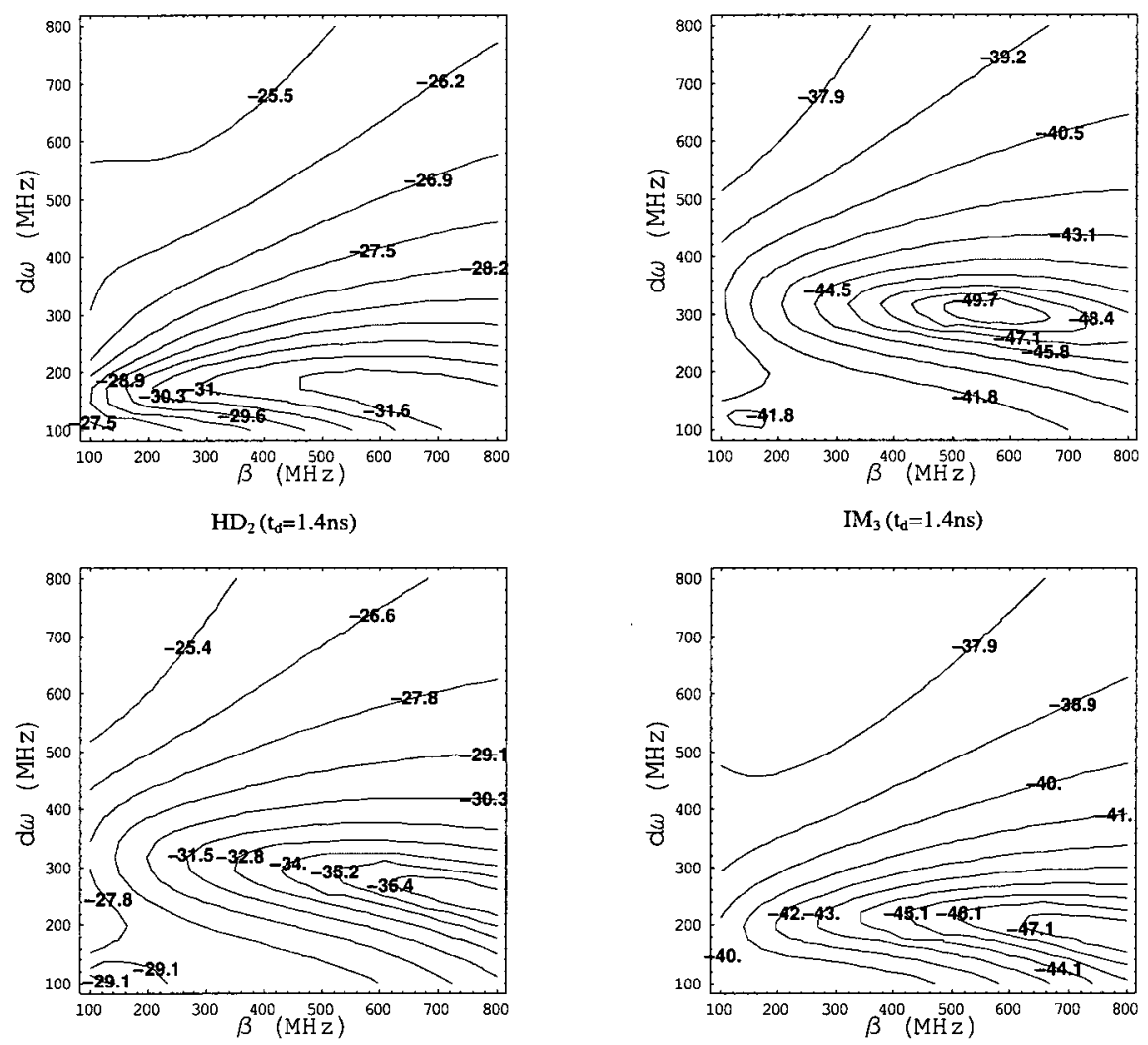

$\mathrm{HD}_{2}\left(\mathrm{t}_{\mathrm{d}}=1.45 \mathrm{~ns}\right)$

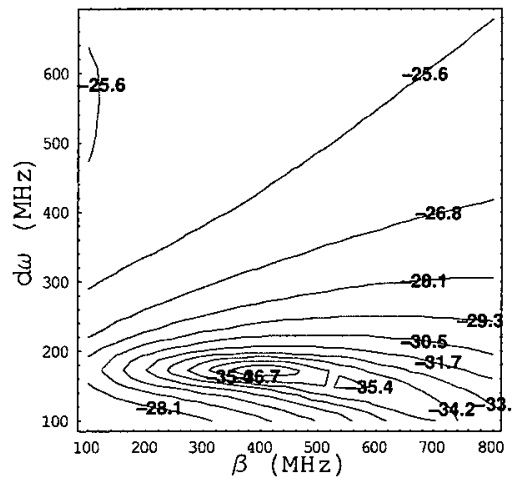

$\mathrm{HD}_{2}\left(\mathrm{t}_{\mathrm{d}}=1.5 \mathrm{~ns}\right)$

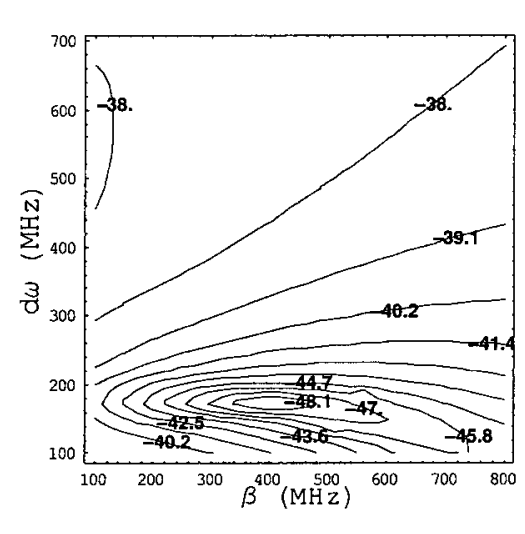

$\mathrm{IM}_{3}\left(\mathrm{t}_{\mathrm{d}}=1.5 \mathrm{~ns}\right)$

Figure B.7: Tank circuit parameters optimization for different linear path delays. 


\section{Appendix C}

\section{Relationship between the Normal Mode Parameters and the Distributed Parameters of the Coupled Transmission Line}

The characteristics of coupled transmission line has been studied in $[84,85]$. The normal mode parameters of an uniform coupled transmission line are related to the distributed parameters of the transmission line by

$$
\begin{aligned}
& Z_{c 1}=\left(\frac{1}{\gamma_{c}}\right) \frac{z_{1} z_{2}-z_{m}^{2}}{z_{2}-z_{m} R_{c}}=\frac{1}{Y_{c 1}} \\
& Z_{c 2}=\left(\frac{R_{c}}{\gamma_{c}}\right) \frac{z_{1} z_{2}-z_{m}^{2}}{z_{1} R_{c}-z_{m}}=\frac{1}{Y_{c 2}} \\
& Z_{\pi 1}=\left(\frac{1}{\gamma_{\pi}}\right) \frac{z_{1} z_{2}-z_{m}^{2}}{z_{2}-z_{m} R_{\pi}}=\frac{1}{Y_{\pi 1}} \\
& Z_{\pi 2}=\left(\frac{R_{c}}{\gamma_{\pi}}\right) \frac{z_{1} z_{2}-z_{m}{ }^{2}}{z_{1} R_{\pi}-z_{m}}=\frac{1}{Y_{\pi 2}}
\end{aligned}
$$

and

$$
\begin{aligned}
& \gamma_{c}^{2}=\frac{a_{1}+a_{2}}{2}+\frac{1}{2}\left(\left(a_{1}-a_{2}\right)^{2}+4 b_{1} b_{2}\right)^{1 / 2} \\
& \gamma_{\pi}^{2}=\frac{a_{1}+a_{2}}{2}-\frac{1}{2}\left(\left(a_{1}-a_{2}\right)^{2}+4 b_{1} b_{2}\right)^{1 / 2}
\end{aligned}
$$




$$
\begin{aligned}
R_{c} & =\frac{\left(a_{2}-a_{1}\right)+\left(\left(a_{2}-a_{1}\right)^{2}+4 b_{1} b_{2}\right)^{1 / 2}}{2 b_{1}} \\
R_{\pi} & =\frac{\left(a_{2}-a_{1}\right)-\left(\left(a_{2}-a_{1}\right)^{2}+4 b_{1} b_{2}\right)^{1 / 2}}{2 b_{1}}
\end{aligned}
$$

where

$$
\begin{aligned}
& a_{1}=y_{1} z_{1}+y_{m} z_{m} \\
& a_{2}=y_{2} z_{2}+y_{m} z_{m} \\
& b_{1}=z_{1} y_{m}+y_{2} z_{m} \\
& b_{2}=z_{2} y_{m}+y_{1} z_{m}
\end{aligned}
$$

and

$$
\begin{gathered}
z_{1}=j \omega L_{1} \\
z_{2}=j \omega L_{2} \\
z_{m}=j \omega L_{m} \\
y_{1}=j \omega C_{1} \\
y_{2}=j \omega C_{2} \\
y_{m}=-j \omega C_{m}
\end{gathered}
$$




\section{Appendix D}

\section{Using Volterra Transfer Function to Calculate the Multi-tone Response of a Nonlinear System}

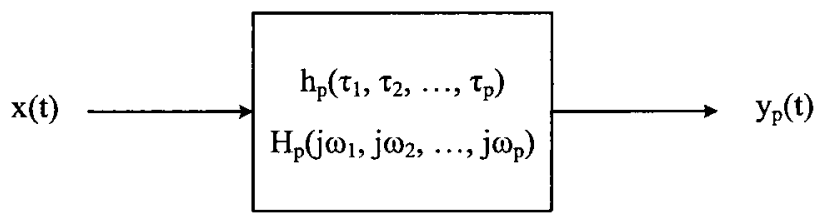

Figure D.1: A $p^{\text {th }}$ order nonlinear system

For a $p^{\text {th }}$ order nonlinear system, its output response can be expressed by

$$
y_{p}(t)=\int_{-\infty}^{\infty} \ldots \int_{-\infty}^{\infty} h_{p}\left(\tau_{1}, \tau_{2}, \ldots \tau_{p}\right) x\left(t-\tau_{1}\right) x\left(t-\tau_{2}\right) \ldots x\left(t-\tau_{p}\right) d \tau_{1} d \tau_{2} \ldots d \tau_{p}
$$

where $h_{p}\left(\tau_{1}, \tau_{2}, \ldots, \tau_{p}\right)$ is the $p^{t h}$ order Volterra kernel of the nonlinear system. The transform of the $p^{\text {th }}$ order Volterra kernel is

$$
H_{p}\left(j \omega_{1}, j \omega_{2}, \ldots j \omega_{p}\right)=\int_{-\infty}^{\infty} \ldots \int_{-\infty}^{\infty} h_{p}\left(\tau_{1}, \tau_{2}, \ldots \tau_{p}\right) e^{-j\left(\omega_{1} \tau_{1}+\omega_{2} \tau_{2}+\ldots+\omega_{p} \tau_{p}\right)} d \tau_{1} d \tau_{2} \ldots d \tau_{p}
$$

The multi-tone input can be expressed as

$$
x(t)=\left(A_{1} e^{j \omega_{1} t}+A_{1}^{*} e^{-j \omega_{1} t}\right)+\left(A_{2} e^{j \omega_{2} t}+A_{2}^{*} e^{-j \omega_{2} t}\right)+\ldots+\left(A_{n} e^{j \omega_{n} t}+A_{n}^{*} e^{-j \omega_{n} t}\right)
$$


Substituting (D.0.3) into (D.0.1) to obtain the output response,

$$
\begin{gathered}
y_{p}(t)=\int_{-\infty}^{\infty} \ldots \int_{-\infty}^{\infty} h_{p}\left(\tau_{1}, \tau_{2}, \ldots \tau_{p}\right) \times \\
\left(\left(A_{1} e^{j \omega_{1}\left(t-\tau_{1}\right)}+A_{1}^{*} e^{-j \omega_{1}\left(t-\tau_{1}\right)}\right)+\ldots+\left(A_{n} e^{j \omega_{n}\left(t-\tau_{1}\right)}+A_{n}^{*} e^{-j \omega_{n}\left(t-\tau_{1}\right)}\right)\right) \\
\left(\left(A_{1} e^{j \omega_{1}\left(t-\tau_{2}\right)}+A_{1}^{*} e^{-j \omega_{1}\left(t-\tau_{2}\right)}\right)+\ldots+\left(A_{n} e^{j \omega_{n}\left(t-\tau_{2}\right)}+A_{n}^{*} e^{-j \omega_{n}\left(t-\tau_{2}\right)}\right)\right) \\
\ldots \\
\left(\left(A_{1} e^{j \omega_{1}\left(t-\tau_{n}\right)}+A_{1}^{*} e^{-j \omega_{1}\left(t-\tau_{n}\right)}\right)+\ldots+\left(A_{n} e^{j \omega_{n}\left(t-\tau_{n}\right)}+A_{n}^{*} e^{-j \omega_{n}\left(t-\tau_{n}\right)}\right)\right) \\
d \tau_{1} d \tau_{2} \ldots d \tau_{p}
\end{gathered}
$$

The multiplication of $x\left(t-\tau_{x}\right)$ can be expanded and written in the form of

$$
\sum^{(2 n)^{p}}\left(A_{x_{1}} A_{x_{2}} \ldots A_{x_{p}}\right) e^{j\left(\omega_{x_{1}}+\omega_{x_{2}}+\ldots+\omega_{x_{p}}\right) t} e^{-j \omega_{x_{1}} \tau_{1}} e^{-j \omega_{x_{2}} \tau_{2}} \ldots e^{-j \omega_{x_{p}} \tau_{p}}
$$

where $x_{i}$ is among $1-n$. Moving $\Sigma$ outside, we have

$$
\begin{aligned}
y_{p}(t)= & \sum^{(2 n)^{p}}\left(A_{x_{1}} A_{x_{2}} \ldots A_{x_{p}}\right) \\
& \int_{-\infty}^{\infty} \ldots \int_{-\infty}^{\infty} h_{p}\left(\tau_{1}, \tau_{2}, \ldots \tau_{p}\right) e^{-j\left(\omega_{x_{1}} \tau_{1}+\omega_{x_{2}} \tau_{2}+. .+\omega_{x_{p}} \tau_{p}\right)} d \tau_{1} d \tau_{2} \ldots d \tau_{p} . \\
& e^{j\left(\omega_{x_{1}}+\omega_{x_{2}}+\ldots+\omega_{x_{p}}\right) t} \\
= & \sum^{(2 n)^{p}}\left(A_{x_{1}} A_{x_{2}} \ldots A_{x_{p}}\right) H_{p}\left(j \omega_{x_{1}}, j \omega_{x_{2}}, \ldots j \omega_{x_{p}}\right) \cdot e^{j\left(\omega_{x_{1}}+\omega_{x_{2}}+\ldots+\omega_{x_{p}}\right) t}
\end{aligned}
$$

which is the sum of output tones at frequencies $\left(\omega_{x_{1}}+\omega_{x_{2}}+\ldots+\omega_{x_{p}}\right)$. Therefore, the output phaser for a given $\left(\omega_{x_{1}}+\omega_{x_{2}}+\ldots+\omega_{x_{p}}\right)$ can be directly obtained as

$$
c \cdot\left(A_{x_{1}} A_{x_{2}} \ldots A_{x_{p}}\right) H_{p}\left(j \omega_{x_{1}}, j \omega_{x_{2}}, \ldots j \omega_{x_{p}}\right)
$$

where $c$ is due to the repeated combination of $\omega_{x}$ in (D.0.6). 


\section{Appendix E}

\section{MatLab Code of the Adaptive Calibration Algorithm}

The appendix contains the main program of the Matlab code implementing the adaptive calibration using multiple variable feedback algorithm. 


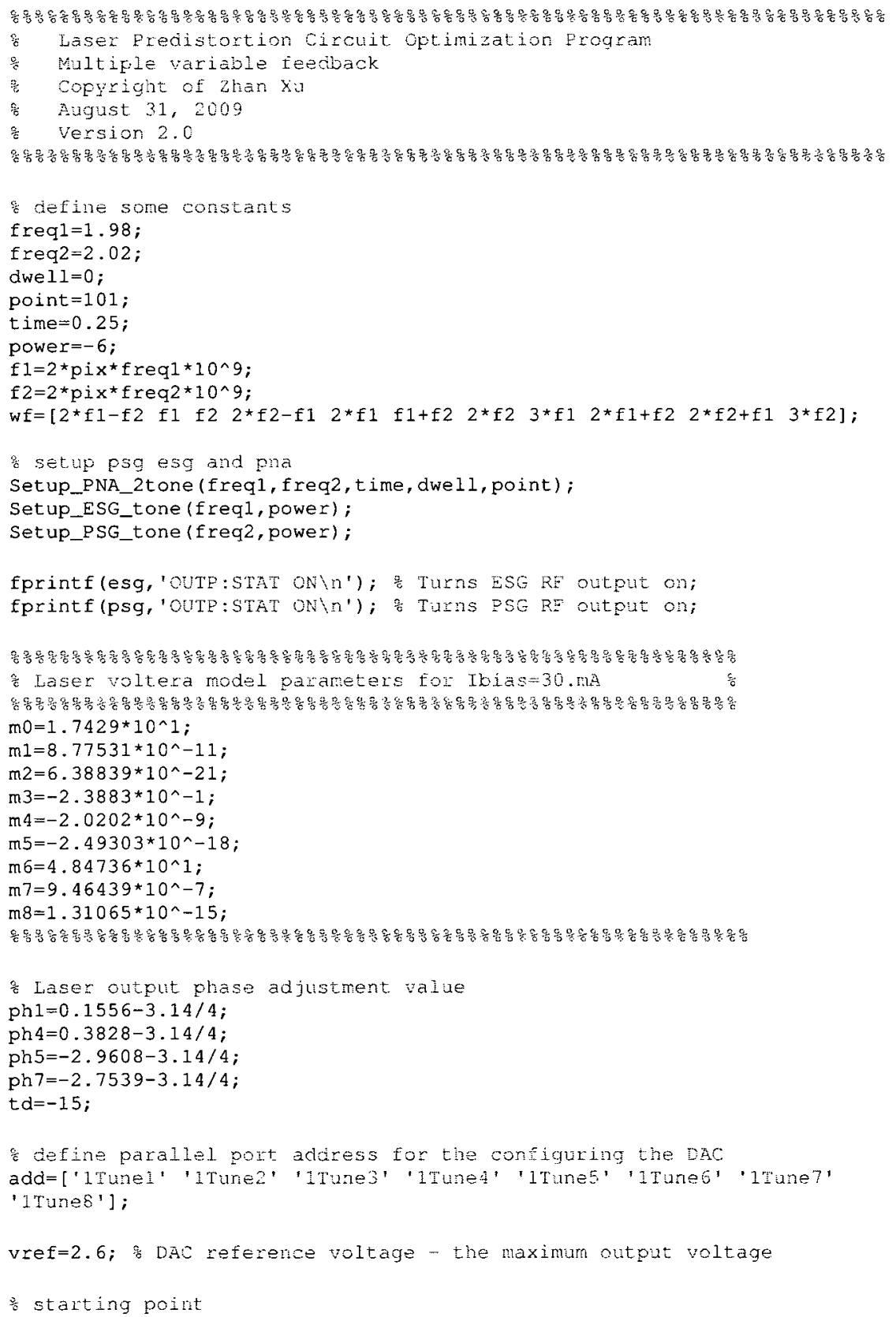




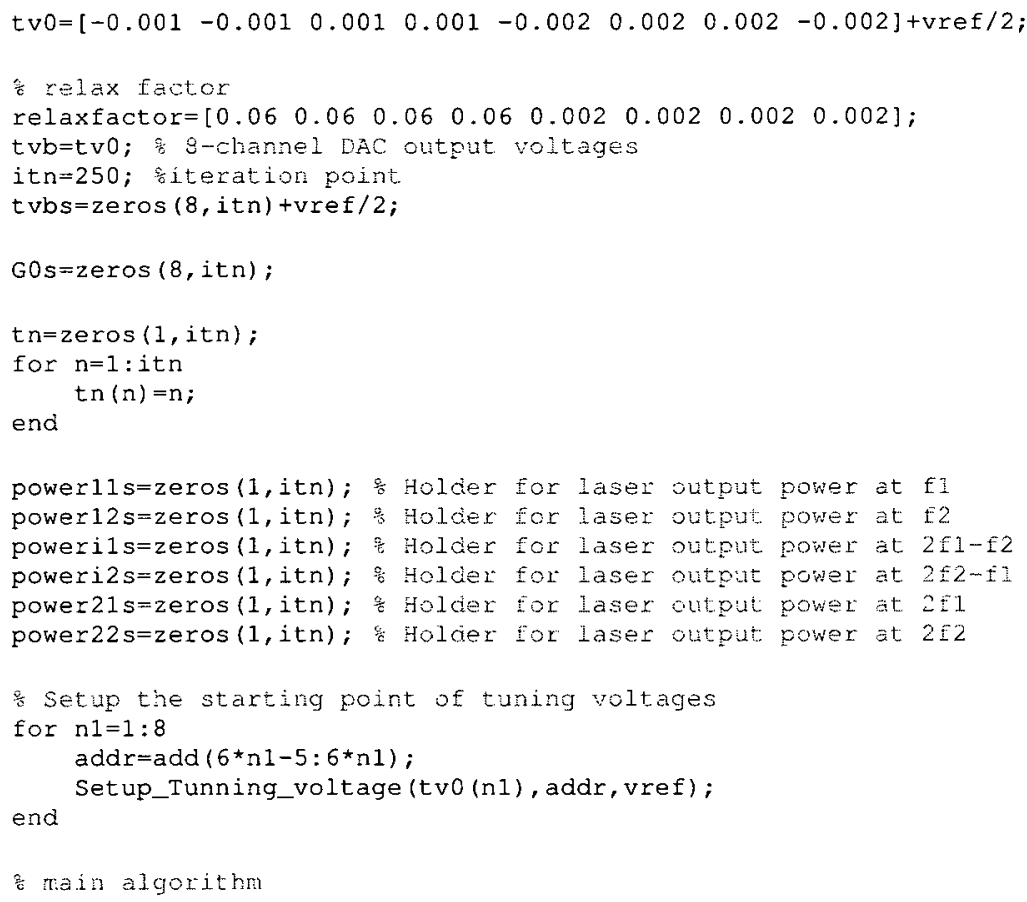

train algorithn

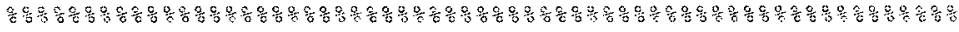
c Measure the linear path signal at port 1 of PNA

[resulta]=Read_PNA_2tone_p1 (point); HDlla=resulta $(7,:)$; HD12a=resulta $(6,:)$; \& Measurement at 12

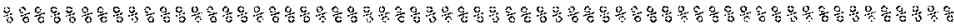
F veasure the predistortion path signal at port 2 of PWA

[resultb]=Read_PNA_2tone_p2 (point) ;

HD1 1b=resultb $(7,:) ;$ Measurerent at fi HD12b=resultb $(6,:)$; \& Measurerent at fo IM31b=resultb $(8,:)$; : Measuiement at $2 \pm 1-f 2$ IM32b=resultb(5,:); \& Measurement at 2f2-t1 HD2 $1 \mathrm{~b}=$ resultb $(4,:) ;$ Meastirement at $2: 1$ $\operatorname{HD} 22 b=r e s u l t b(3,:) ;$ \& Measurement at 212 IM2 $2 b=$ resultb $(9,:)$; Measlirerent at $f 1+f 2$ HD $31 b=r e s u l t b(2,:)$; $c$ Measuierent. at $3 \mp 1$ HD $32 b=$ resultb $(1,:)$; seasurement at $3 \pm 2$ HD $33 b=$ resultb $(10,:)$; : Measurement at $2 f 1+f 2$ HD $34 b=$ resultb $(11,:)$; $\%$ Measurement at $2 f 2+f 1$ 


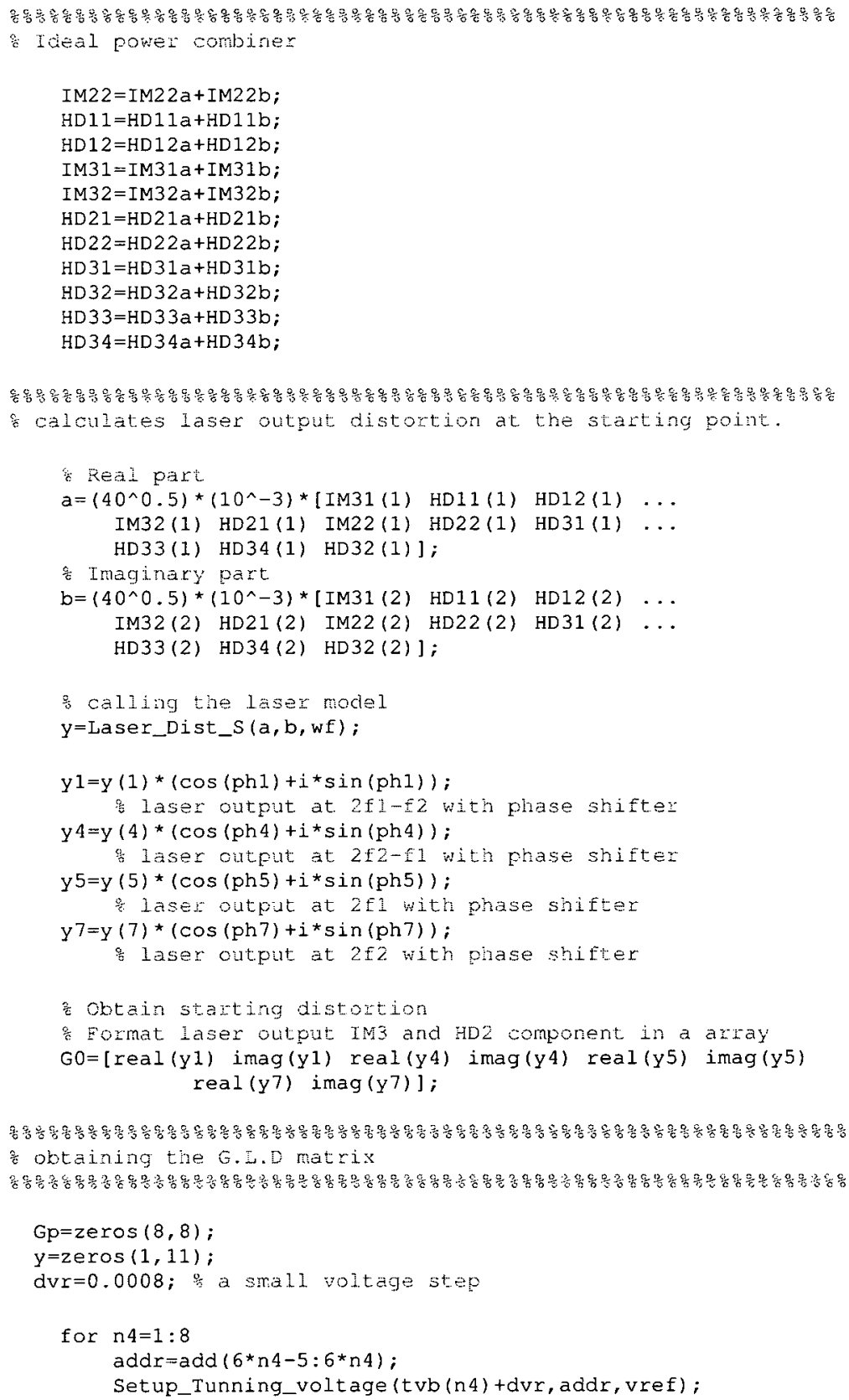


small increnent for each tuning voltage

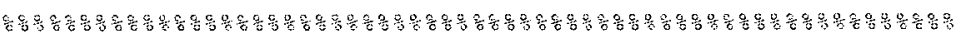

3 Measure the predistortion path signal at port 2 of PNA

[resultb]=Read_PNA_2tone_p2 (point);

HD $11 \mathrm{~b}=$ resultb $(7,:)$; 5 Measurement at 11

HD $12 \mathrm{~b}=$ resultb $(6,:)$; 8 Measurement at 12

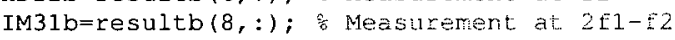

IM $32 \mathrm{~b}=$ resultb $(5,:) ; 3$ Measurement at $2 \hat{1} 2-\mathbf{1}$

HD2 $1 b=r e s u l t b(4,:) ;:$ Measurement at $2 f 1$

HD22b=resultb $(3,:) ;$ क Measurement at $2 \mathrm{fz}$

IM2 $2 \mathrm{~b}=$ resultb $(9,:) ;$; Measurement at fl+f2

HD $31 \mathrm{~b}=$ resultb $(2,:)$; \% Measurement at. $3 \mathrm{fl}$

HD $32 \mathrm{~b}=$ resultb $(1,:) ;$; Measurement at 312

HD $33 \mathrm{~b}=$ resultb $(10,:)$; ? Measurement at $a f 1+f 2$

HD $34 b=$ resultb $(11,:) ;$ seasurement at $2 t 2+f 1$

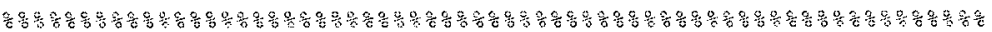

s. IClal power combine

IM2 2 =IM2 $2 a+I M 22 b ;$

HD $11=$ HD $11 a+H D 11 b$;

$\mathrm{HD} 12=\mathrm{HD} 12 \mathrm{a}+\mathrm{HD} 12 \mathrm{~b}$

IM31 = IM31a+IM31b;

$\operatorname{IM} 32=\operatorname{IM} 32 \mathrm{a}+\mathrm{IM} 32 \mathrm{~b}$;

$\mathrm{HD} 21=\mathrm{HD} 21 \mathrm{a}+\mathrm{HD} 21 \mathrm{~b}$

$\mathrm{HD} 22=\mathrm{HD} 22 \mathrm{a}+\mathrm{HD} 22 \mathrm{~b}$;

$\mathrm{HD} 31=\mathrm{HD} 31 \mathrm{a}+\mathrm{HD} 31 \mathrm{~b}$

$\mathrm{HD} 32=\mathrm{HD} 32 \mathrm{a}+\mathrm{HD} 32 \mathrm{~b}$

HD $33=\mathrm{HD} 33 \mathrm{a}+\mathrm{HD} 33 \mathrm{~b}$;

HD $34=H D 34 a+H D 34 b$;

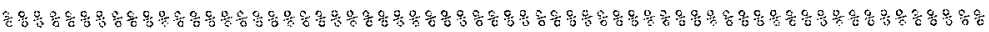

calculatirg laser output distortion for smal change of each

o tuning voltage.

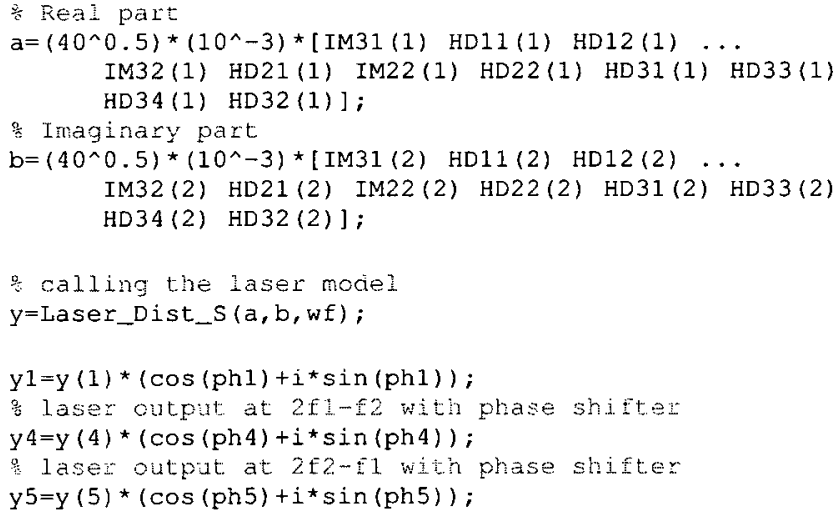




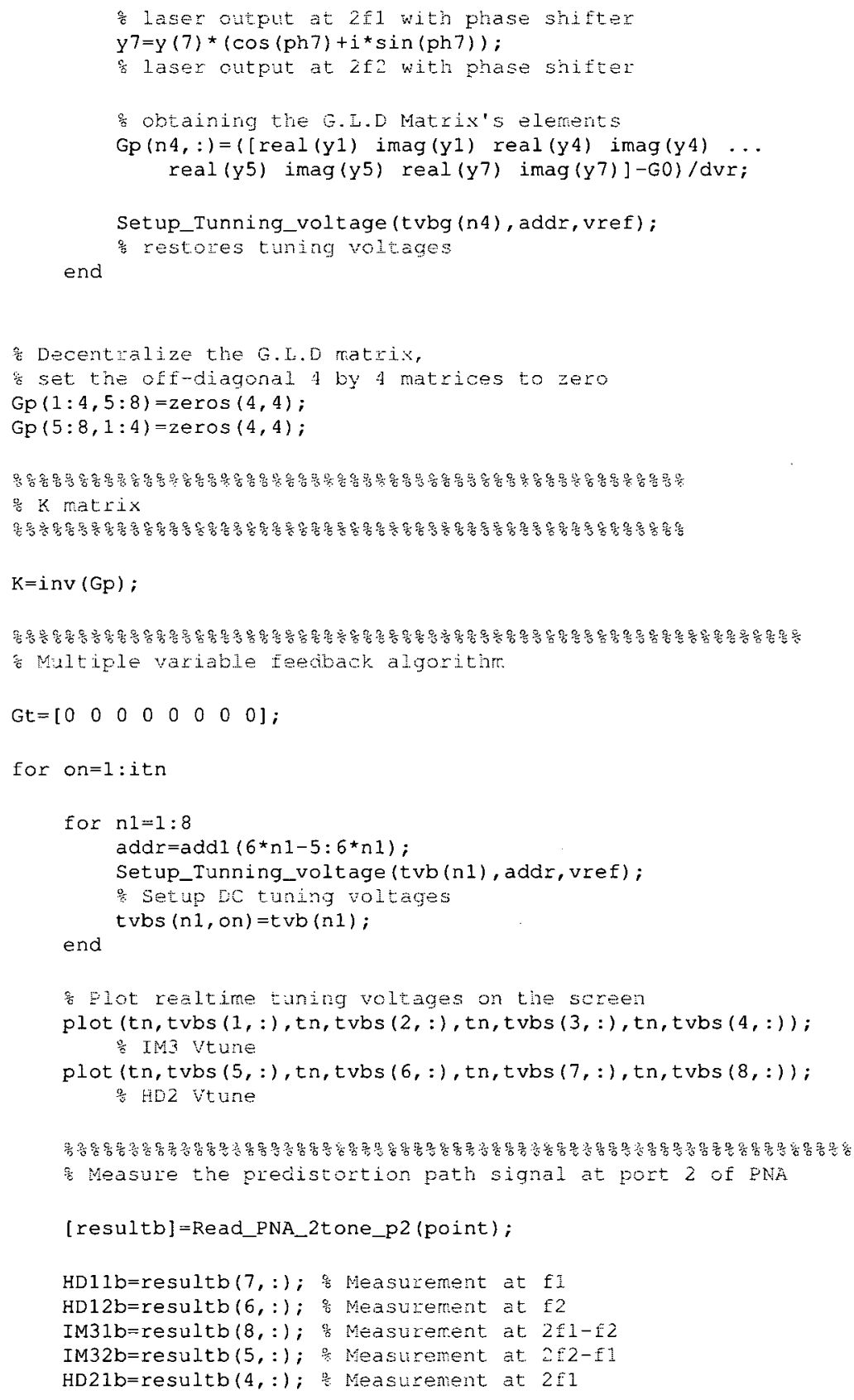




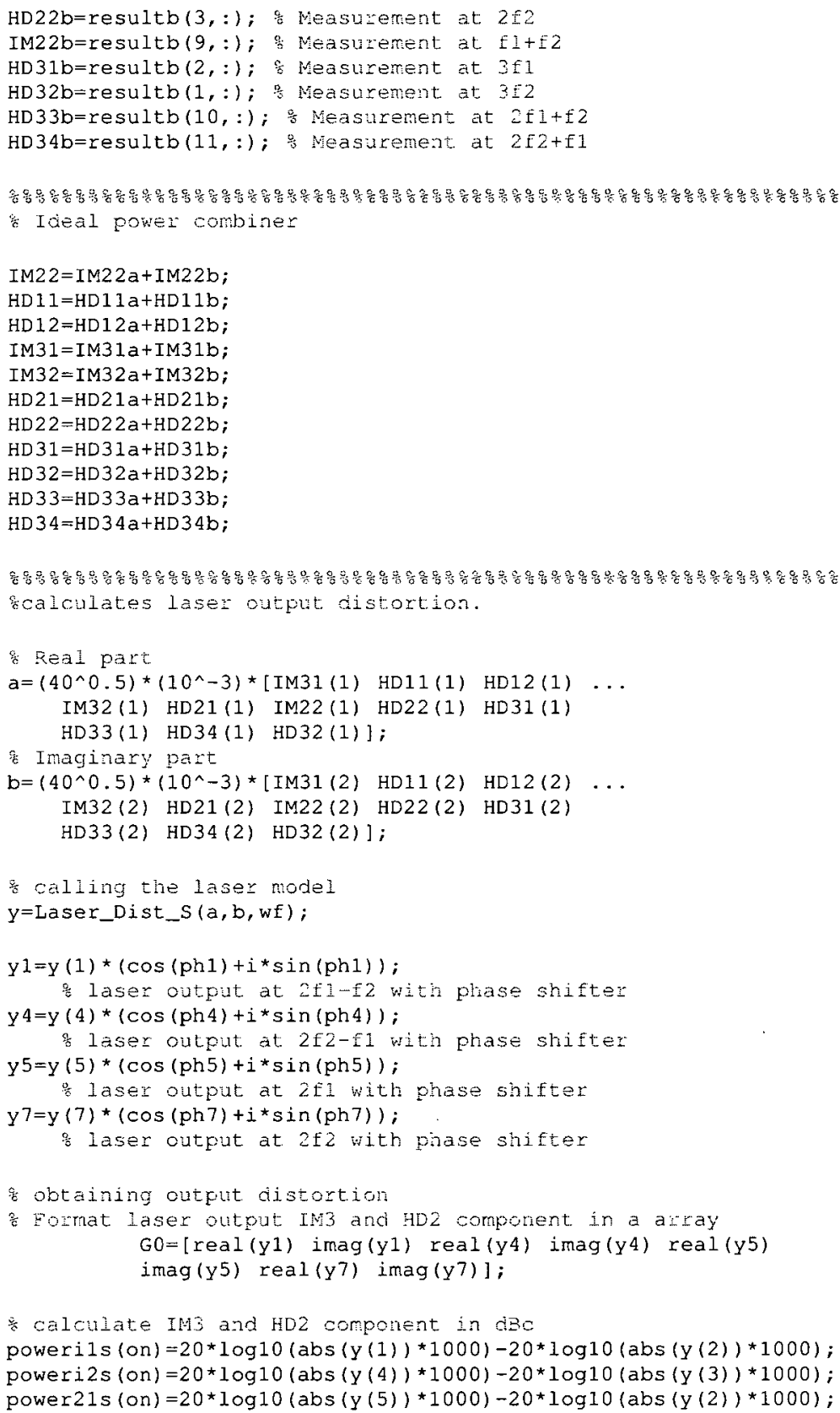


power $22 \mathrm{~s}(\mathrm{on})=20 * \log 10(\mathrm{abs}(\mathrm{y}(7)) * 1000)-20 * \log 10(\mathrm{abs}(\mathrm{y}(3)) * 1000)$;

clot realtime $I M 3$ and $H D 2$ on the screen

plot (tn, poweri1s,tn, poweri2s); INi3

plot (tn, power21s,tn, power22s);

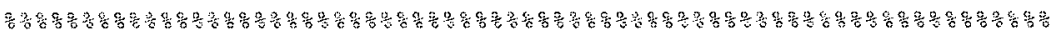

\& Loop filter a/s,

定 integration in CT or accumulator in DT and negative feedback

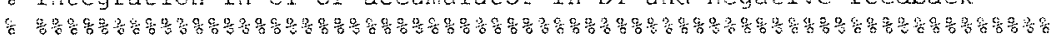

$G t=G t-G 0$ * relaxfactor; \& Accumlator and negative feedback

$\mathrm{dvb}=\mathrm{t}$ ranspose $\left(\mathrm{K}^{\star}\right.$ transpose $\left.(\mathrm{Gt})\right)$;

tvb=tv0+dvb; sopdate the tuning voltage

end

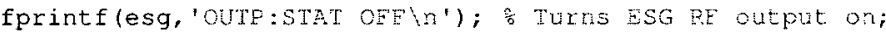

fprintf (psg, OUTE:STAT OFE'n'); B Turns PSG RE output on;

Close_ESG;

Close_PNA; 


\section{Bibliography}

[1] M. Rajesh, B. Inder and B. Prakash, RF and Microwave Coupled-line Circuits. Artech House, TK7876.M634, 1999.

[2] H. Al-Raweshidy and S. Komaki, Radio over Fiber Technologies for Mobile Communications Networks. Artech House Inc. Boston and London, ISBN 1-58053-148-2, pp 148-239, 2002.

[3] Winston I. Way, "Optical Fiber-based Microcellular Systems: an Overview," IEICE Transactions on Communications, 1993.

[4] X. N. Fernando and A. B. Sesay, "Higher Order Adaptive Filter Based Predistortion for Nonlinear Distortion Compensation of Radio over Fiber Links," IEEE International Conference on Communications, vol. 1, pp. 367-371, 2000.

[5] T. Iwai, K. Sato, and K.-I. Suto, "Signal Distortion and Noise in AM-SCM Transmission System Employing the Feedforward Linearization MQW-EW External Modulator," IEEE Journal of Lightwave Technology, vol. 13, pp. 1606-1612, August 1995.

[6] B. Buxton and R. Vahldieck, "Noise and Intermodulation Distortion Reduction in an optical Feedforward Transmitter," IEEE MTT-S Digest, pp. 1105-1108, 1994.

[7] D. Hassin and R. Vahldieck, "Feedforward Linearization of Analog Modulated Laser Diodes: Theoretical Analysis and Experimental Verification," IEEE ESSCIRC Conference, December 1993.

[8] L. S. Fock and R. S. Tucker, "Reduction of Distortion in Analog Modulated Semiconductor Lasers by FeedForward Compensation," Elect. Lett., April 1991.

[9] D. Hassin and R. Vahldieck, "Improved Feedforward Linearization of Laser Diodes - Simulation and Experimental Results," IEEE MTT-S Digest, 1993.

[10] L. S. Fock and R. S. Tucker, "Simultaneous Reduction of Intensity Noise and Distortion in Semiconductor Lasers by FeedForward Compensation," Elect. Lett., July 1991.

[11] K. Tsukamoto, S. Fujii, P. Sanjo, and S. Komaki, "Fiber-optic Microcellular Communication System using Frequency Modulated LD and Optical Discriminator and its Nonlinear Distortion Suppression," IEEE PIMRC'97, 1997.

[12] J. Tsimbinos and K. V. Lever, "Nonlinear Compensation using Orthogonal Inverses: Broadband Inputs and Robustness Issues," TENCON' '96, 1996.

[13] - "The Computational Complexity of Nonlinear Compensators Based on the Volterra Inverse," 8th IEEE Sig. Process. Conf., 1996.

[14] Y. Ueno and M. Kajitani, "Color TV Transmission using Light Emitting Diode," NEC, 1974.

[15] H. Gysel and M. Ramachandran, "Electrical Predistortion to Compensate for Combined Effect of Laser Chirp and Fiber Dispersion," Elect. Lett., Feburary 1991. 
[16] L. Roselli and et al, "Predistortion Circuit Design for II and III Order Simultaneous Linearization in Multiservice Telecommunications Apparatuses," IEEE MTT-S Digest, 2002.

[17] Y. Chiu and et al., "Broadband Electronic Linearizer for Externally Modulated Analog Fiber-Optic Links," IEEE Phot. Tech. Lett., January 1999.

[18] X. J. Meng, A. Yacoubian, and J. H. Bechtel, "Electro-optical Predistortion Technique for Linearization of Mach-Zehnder Modulators," Elec. Lett, December 2001.

[19] G. C. Wilson, "Optimized Predistortion of Over Modulated Mach-Zehnder Modulators with Multicarrier Input," IEEE Phot. Tech. lett., December 1997.

[20] A. V. de Grijp and et al., "Novel Electro-Optical Feedback Technique for Noise and Distortion Reduction in High-Quality Analog Optical Transmission Video Signals," Elec. Lett., May 1981.

[21] M. Sekita and et al, "TV Video Transmission by Analog Baseband Modulation of 1.3 um Band Laser Diode," 6th Europ. Conf. Opt. Comm., 1980.

[22] L. S. Fock and R. S. Tucker, "Simultaneous Reduction of Intensity Noise and Distortion in Semiconductor Lasers by FeedForward Compensation," Elect. Lett., July 1991.

[23] L. Roselli and et al., "Analog Laser Predistrotion For Multiservice Radio-Over-Fiber Systmes," IEEE Journal of Lightwave Technology, 2003.

[24] S. Ghoniemy, L. MacEachern, and S. Mahmoud, "Semiconductor Laser Distortion Improvement for High Performance Analog Optical Transmission," 46 IEEE MWSCAS, 1993.

[25] L. Roselli, V. Borgioni, F. Zepparelli, and F. Ambrosi, "Analog Laser Predistortion for Multiservice Radio-over-fiber Systems," Journal of Lightwave Technology, vol. 21, pp. 1211-1223, May 2003.

[26] Zhan Xu and Leonard MacEachern, "A Predistortion Circuit Design Technique for High Performance Analogue Optical Transmission," IEEE Micro-system and Nano-electronics Research Conference, pp. 213-216, October 2008.

[27] _ "Multiple Stage Uncompensated Marchand Balun with 7:1 Bandwidth Ratio on Double Side PCB," Electronics Letters, May 2009.

[28] — "Optimum Design of Wideband Compensated and Uncompensated Marchand Baluns with Step Transformers," IEEE Transactions on Microwave Theory and Techniques, August 2009.

[29] — "Adaptive Predistortion of Lasers Using Multiple-variable-feedback Algorithm," Electronics Letters, September 2009.

[30] G. P. Agrawal and N. K. Dutta, Semiconductor Lasers. vol. 1. Van Nostrand Reinhold, 1993.

[31] K. Lizuka, Elements of Photonics. Wiley-Interscience, Volume II,2002.

[32] G. P. Agrawal, Fiber-Optic Communication Systems. John Wiley and Sons Inc., 2002.

[33] J. Carroll, J. Whiteaway, and D. Plumb, Distributed FeedBack Semiconductor Lasers. vol. PM52 of SPIE. Michael Faraday House, Six Hills Way, UK: IEE, 1998. ISBN 0852969171.

[34] G. Keiser, Optical Fiber Communications. McGraw-Hill Higher Education, Thomas Casson, third ed., 2000.

[35] S. Ghoniemy, RF/Fiber Optical Interface for Microcellular Wireless Transceivers. Thesis, Carleton University, 2003.

[36] S. Ghoniemy, L. MacEachern, and S. Mahmoud, "Large Signal Modeling for Analog Communications," Proc. SPIE, vol. TD01, pp. 139-141, May 2002. 
[37] S. A. Javro and S. M. Kang, "Transforming Tucker's Linearized Laser Rate Equation to a Form taht Has a Single Solution Regime," IEEE Jurnal Of Light Wave Technology, September 1995.

[38] G. P. Agrawal, "Specral Hole-Burning and Gain Saturation in Semiconducto Lasers: Strong-Signal Theory," Jurnal of App. Phy., Feburary 1988.

[39] C. Y. Tsai and et al., "Nonlinear Gain Coefficient in Semiconductor Lasers: Efect of Carrier Heating," IEEE JQE., Feburary 1996.

[40] C.-Y. Tsai and et al., "Carrier Density Dipinning above Threshold in Semiconductor Lasers: Efect of Carrier Heating and Spectral Hole Burning," IEE Proc. Optoelectronics, August 1997.

[41] N. Bewtra and et al, "Modeling of Quantum Well Lasers with Electro-Opto-Thermal Interactions," IEEE JQE, June 1995.

[42] I. M. P. Aarts and E. H. Sargent, "Above-Threshold Leakage in Semiconductor Lasers: An Analytical Physical Model," IEEE JQE., April 2000.

[43] P. Wambacq and W. Sansen, Distortion Analysis of Analog Integrated Circuits. Kluwer Academic Publishers, Boston, 1998.

[44] M. Sekita, T. Kawamura and et al., "TV Video Transmission by Analog Baseband Modulation of 1.3 um Bband Laser Diode," 6th European Conference Opt. Comm., pp. 394-396, York, 1980.

[45] A. Van de Grijp, J. C. Koopman and et al, "Novel Electro-optical Feedback Technique for Noise and Distortion Reduction in High-quality Analog Optical Transmission Video Signals," Electronics Letters, vol. 17, pp. 361-362, May 1981.

[46] — - "Linearized Transmitters for Optical Communications," Proceedings of IEEE Conference on Circuits and Systems, pp. 288-292, April 1977.

[47] X. J. Meng, T. Chau and et al, "Suppression of Second Harmonic Distortion in Directly Modulated Distributed Feedback Lasers by External Light Injection," Electronics Letters, pp. 2040-2041, Oct. 1998.

[48] X. J. Meng, D. Tong and et al, "Demonstration of an Analog Fiber-optic Link Employing a Directly Modulated Lasers with External Light Injection," IEEE Phot. Tech. Letters, pp. 1620-1622, Nov. 1998.

[49] G. Yabre and J. Le Bihan, "Reduction of Nonlinear Distortion in Directly Modulated Semiconductor Lasers by Coherent Light Injection," IEEE Journal of Quantum Electronics, pp. 1132-1140, July 1997.

[50] X. N. Fernando and A. B. Sesay, "Adaptive Asymmetric Linearization of Radio over Fiber Links for Wireless Access," IEEE Transactions on Vehicular Technology, vol. 51, pp. 1576-1586, November 2002 .

[51] A. Lidgard and N. A. Olsson, "Generation and Cancellation of Second-order Harmonic Distortion in Analog Optical Systems by Interfermetric FM-AM Conversion," IEEE Photonics Technology Letters, vol. 2, pp. 519-521, July 1990.

[52] K. Asatani, T. Kimura, "Linearization of LED Nonlinearity by Predistortion," IEEE Transactions on Electronic Devices, vol. ED-25, pp. 207-212, Feb. 1978.

[53] R. B. Childs, V. A. O'Byrne, "Predistortion Linearization of Directly Modulated DFB Lasers and External Modulators for AM Video Transmission," Proceedings Optical Fiber Communications Conference, Jan. 1990.

[54] F. Shearer, L. MacEachern, "A Precision CMOS Analog Cubing circuit," IEEE NEWCAS on Circuits and Systems, June 2004. 
[55] S. Tanaka, N. Taguchi, and et al, "A Predistortion-type Equi-path Linearizer Designed for Radioon-fiber System," IEEE Transactions on Microwave Theory and Techniques, vol. 54, pp. 938-944, February 2006.

[56] X. N. Fernando and A. B. Sesay, "Look-Up Table Based Adaptive Predistortion for Dynamic Range Enhancement in a Radio over Fiber Links," IEEE Conf. on Comm. Comp. and SP, vol. 1, pp. 26-29, 1999.

[57] G. Steiner, S. Hunziker, and W. Baechtold, "Reduction of 3rd Order Intermodulation of a Semiconductor Laser by an Adaptive Low-Cost Predistortion Circuit at 1.8 GHz," IEEE LEOS Meeti RF Phot. for CATV and HFC Sys., July 1999.

[58] R. Sadhwani and B. Jalali, "Adaptive CMOS Predistortion Linearizer for Fiber-optic Links," IEEE Journal of Lightwave Technology, vol. 21, pp. 3180-3193, December 2003.

[59] Behazad Razavi, RF Microelectronics. Prentice Hall PTR, 1998.

[60] William Liu, MOSFET Models for SPICE Simulation, Including BSIM3v3 and BSIM4. John Wiley and Sons Inc., 2001.

[61] T. H. Lee, The Design of CMOS Radio-Frequency Integrated Circuits. Cambridge University Press, 1998.

[62] B. Gilbert, "The Multi-tanh Principle: a Tutorial Overview," IEEE Journal of Solid-State Circuits, January 1998.

[63] G. C. Wilson and T. H. Wood and et al, "Predistortion of Electroabsorption Modulators for Ana$\log$ CATV Systems at $1.55 \mathrm{um}$, " IEEE Journal of Lightwave Technology, vol. 15, pp. 1654-1661, September 1997.

[64] G. Steiner, S. Hunziker and et al, "Reduction of 3rd Order Intermodulation of a Semiconductor laser by an Adaptive Low-Cost Predistortion Circuit at $1.8 \mathrm{GHz}$," IEEE Digest of the LEOS Summer Topical Meetings, pp. IV43-IV44, July 1999.

[65] Mathematica Help Documentation. Wolfram Research.

[66] N. Marchand " "Transmission Line Conversion Transformers," Electronics, vol. 17, pp. 142-145, December 1944.

[67] D. M. Pozar, Microwave Engineering. Addison-Wesley, ISBN 0-201-50418-9, TK7876.P69, 1990.

[68] R. Bawer and J. J. Wolfe, "A Printed-Circuit Balun for Use with Spiral Antennas," IRE Transactions Microwave Theory and Techniques, May 1960.

[69] C. L. Goldsmith and etc., "Synthesis of Marchand Baluns Using Multilayer Microstrip Strucures," Int. J. of Mricrowave and Millimeter-Wave Computer-Aided Engineering, July 1992.

[70] J. W. McLaughlin, D. A. Dunn, and R. W. Grow, "A Wide-band Balun," IRE Transactions Microwave Theory and Techniques, July 1958.

[71] K. Nishikawa, I. Toyoda, and T. Tokumitsu, "Compact and Broad-band Three-Dimensional MMIC Balun," IEEE Transactions on Microwave Theory and Techniques, January 1999.

[72] R. G. Freitag, "A Unified Analysis of MMIC Power Amplifier Stability," IEEE MTT-S International Microwave Symposium Digest, June 1992.

[73] K. T. Vijai , "Asymmetric Coupled Transmission Lines in an Inhomogeneous Medium," IEEE Transactions on Microwave Theory and Techniques, September 1975.

[74] C.-M. Tsai and K. C. Gupta, "A Generalized Model for Coupled Lines and its applications to TwoLayer Planar Circuits," IEEE Transactions on Microwave Theory and Techniques, December 1992. 
[75] N.A.Thacker and T.F.Cootes, Vision Through Optimization - Theory: Optimization Methods. http : //homepages.inf.ed.ac.uk/rbf/CVonline/LOCAL - COPIES/BMVA96Tut/node14.html, August, 2009.

[76] R. Fletcher, Practical Methods of Optimization. John Wiley and Sons, 2nd edition, May 2000,ISBN 0471494631.

[77] S. Skogestad and I. Postlethwaite, Multivariable Feedback Control, Analysis and Design. John Wiley and Sons, 2nd Edition, August,2001.

[78] "Amplifier and CW Sweept Intermodulation Distortion Measurements," Agilent PNA Microwave Network Analyzers, Application Note 1408-9.

[79] "Amplifier Swept-Harmonic Measurements," Agilent PNA Microwave Network Analyzers, Application Note 1408-8.

[80] R. Schaumann, M. S. Ghausi and K. R. Laker, Design of Analog Filters, Passive, Active RC and Wsitched Capacitor. Prentice Hall., 1990.

[81] Y. X. Guo, Z. Y. Zhang and L. C. Ong, "LTCC Full-Matching Marchand Balun," IEEE Microwave Conference, 36th European, pp. 76-78, September 2006.

[82] Y. J. Yoon, Y. Lu and et. al, "Design and Characterization of Multilayer Spiral Transmission-line Baluns," IEEE Transactions on Microwave Theory and Techniques, vol. 47, pp. 1841-1846, September 1999.

[83] V. Chande and P. G. Poonacha, "On Neural Networks for Analog to Digital Conversion," IEEE Transactions on Neural Networks, vol. 6, pp. 1269-1274, September 1995.

[84] M. K. Krage, and G. I. Haddad, "Characteristics of Coupled Microstrip Tranmission Lines-I: CoupledMode Formulation of Inhomogeneous Lines," IEEE Transactions on Microwave Theory and Techniques, April 1970.

[85] —-, "Characteristics of Coupled Microstrip Tranmission Lines-I: Evaluation of Coupled-Line Parameters," IEEE Transactions on Microwave Theory and Techniques, April 1970. 